\title{
Modeling and forecasting the spread of COVID-19 with stochastic and deterministic approaches: Africa and Europe
}

\author{
Abdon Atangana ${ }^{1,2^{*}}$ and Seda Iğret Araz $^{3}$
}

*Correspondence:

AtanganaA@ufs.ac.za

1 Institute for Groundwater Studies,

Faculty of Natural and Agricultural

Sciences, University of the Free

State, Bloemfontein, South Africa

${ }^{2}$ Department of Medical Research,

China Medical University Hospital,

China Medical University, Taichung,

Taiwan

Full list of author information is

available at the end of the article

\begin{abstract}
Using the existing collected data from European and African countries, we present a statistical analysis of forecast of the future number of daily deaths and infections up to 10 September 2020. We presented numerous statistical analyses of collected data from both continents using numerous existing statistical theories. Our predictions show the possibility of the second wave of spread in Europe in the worse scenario and an exponential growth in the number of infections in Africa. The projection of statistical analysis leads us to introducing an extended version of the well-blancmange function to further capture the spread with fractal properties. A mathematical model depicting the spread with nine sub-classes is considered, first converted to a stochastic system, where the existence and uniqueness are presented. Then the model is extended to the concept of nonlocal operators; due to nonlinearity, a modified numerical scheme is suggested and used to present numerical simulations. The suggested mathematical model is able to predict two to three waves of the spread in the near future.
\end{abstract}

Keywords: Statistical analysis; Extended blancmange function; Stochastic model; COVID-19 spread with waves; Modified numerical scheme

\section{Introduction}

Interdisciplinary research is the way forward for mankind to be in control of its environment. Of course they will not be able to have total control since the nature within which they live is full of uncertainties, many complex phenomena that have not been yet understood with the current collections of knowledge and technology. For example, we cannot explicitly and confidently explain what is happening at the Bermuda Triangle, although many studies have been done around this place, some believe it is a devil's triangle. There are many other natural occurrences that could not be explained so far with our knowledge. But it has been proven that putting together several concepts from different academic fields could provide better results. COVID-19 is an invisible enemy that left humans with no choice than to put all their efforts from all backgrounds with the aim to protect the survival of their kind. Many souls have been taken, many humans have been infected and some recovered, but still the spread has not yet reached its peak in many countries. While

(c) The Author(s) 2021. This article is licensed under a Creative Commons Attribution 4.0 International License, which permits use sharing, adaptation, distribution and reproduction in any medium or format, as long as you give appropriate credit to the original author(s) and the source, provide a link to the Creative Commons licence, and indicate if changes were made. The images or other third party material in this article are included in the article's Creative Commons licence, unless indicated otherwise in a credit line to the material. If material is not included in the article's Creative Commons licence and your intended use is not permitted by statutory regulation or exceeds the permitted use, you will need to obtain permission directly from the copyright holder. To view a copy of this licence, visit http://creativecommons.org/licenses/by/4.0/. 
in some countries the curve of daily new infected has nearly reached zero, in others the spread is increasing exponentially. For some statistical analysis, we investigated daily cases of infections and deaths due to the COVID-19 spread that occurred in 54 countries in the European continent and 47 countries in the African continent from the beginning of the outbreak to 15 June 2020. To do this, we used the available data on the website of the World Health Organization (WHO) [1,2]. Although mathematicians cannot provide vaccine or cure the disease in an infected person, they can use their mathematical tools to foresee what could possibly happen in the near future with some limitations [3-14]. With the new trend of spread, it is possible that the world will face a second wave of COVID-19 spread, this will be the aim of our work.

The paper is organized as follows. In Sect. 2, we present the definitions of differential and integral operators where singular and nonsingular kernels are used. In Sect. 3, the parameter estimations are presented for the infected and deaths in Africa and Europe using the Box-Jenkins model. In Sect. 4, the simulations for smoothing method for the infected and deaths in Africa and Europe are presented. In Sect. 5, the predictions about the cases of infections and deaths in Africa and Europe are provided. In Sect. 6, we give an analysis of COVID-19 spread based on fractal interpolation and fractal dimension. In Sect. 7, existence and uniqueness for a mathematical model with stochastic component are investigated. Also the numerical simulations for such a model are depicted. In Sect. 8, we present a modified scheme based on the Newton polynomial. In Sect. 9, we provide numerical solutions for the suggested COVID-19 model with different differential operators.

\section{Differential and integral operators}

In this section, we present some definitions of differential and integral operators with singular and nonsingular kernels. The fractional derivatives with power-law, exponential decay, and Mittag-Leffler kernel are given as follows:

\section{Definition 1}

$$
\begin{aligned}
& { }_{0}^{C} D_{t}^{\alpha} f(t)=\frac{1}{\Gamma(1-\alpha)} \int_{0}^{t} \frac{d}{d \tau} f(\tau)(t-\tau)^{-\alpha} d \tau, \\
& { }_{0}^{C F} D_{t}^{\alpha} f(t)=\frac{M(\alpha)}{1-\alpha} \int_{0}^{t} \frac{d}{d \tau} f(\tau) \exp \left[-\frac{\alpha}{1-\alpha}(t-\tau)\right] d \tau, \\
& { }_{0}^{A B C} D_{t}^{\alpha} f(t)=\frac{A B(\alpha)}{1-\alpha} \int_{0}^{t} \frac{d}{d \tau} f(\tau) E_{\alpha}\left[-\frac{\alpha}{1-\alpha}(t-\tau)^{\alpha}\right] d \tau .
\end{aligned}
$$

The fractional integrals with power-law, exponential decay, and Mittag-Leffler kernel are given as follows:

$$
\begin{aligned}
& { }_{0}^{C} J_{t}^{\alpha} f(t)=\frac{1}{\Gamma(\alpha)} \int_{0}^{t}(t-\tau)^{\alpha-1} f(\tau) d \tau, \\
& { }_{0}^{C F} J_{t}^{\alpha, \beta} f(t)=\frac{1-\alpha}{M(\alpha)} f(t)+\frac{\alpha}{M(\alpha)} \int_{0}^{t} f(\tau) d \tau, \\
& { }_{0}^{A B} J_{t}^{\alpha, \beta} f(t)=\frac{1-\alpha}{A B(\alpha)} f(t)+\frac{\alpha}{A B(\alpha) \Gamma(\alpha)} \int_{0}^{t}(t-\tau)^{\alpha-1} f(\tau) d \tau .
\end{aligned}
$$


The fractal-fractional derivatives with power-law kernel, exponential decay, and MittagLeffler kernel are given as follows:

$$
\begin{aligned}
& { }_{0}^{F F P} D_{t}^{\alpha, \beta} f(t)=\frac{1}{\Gamma(1-\alpha)} \frac{d}{d t^{\beta}} \int_{0}^{t} f(\tau)(t-\tau)^{-\alpha} d \tau, \\
& { }_{0}^{F F E} D_{t}^{\alpha, \beta} f(t)=\frac{M(\alpha)}{1-\alpha} \frac{d}{d t^{\beta}} \int_{0}^{t} f(\tau) \exp \left[-\frac{\alpha}{1-\alpha}(t-\tau)\right] d \tau, \\
& { }_{0}^{F F M} D_{t}^{\alpha, \beta} f(t)=\frac{A B(\alpha)}{1-\alpha} \frac{d}{d t^{\beta}} \int_{0}^{t} f(\tau) E_{\alpha}\left[-\frac{\alpha}{1-\alpha}(t-\tau)^{\alpha}\right] d \tau,
\end{aligned}
$$

where

$$
\frac{d f(t)}{d t^{\beta}}=\lim _{t \rightarrow t_{1}} \frac{f(t)-f\left(t_{1}\right)}{t^{2-\beta}-t_{1}^{2-\beta}}(2-\beta) .
$$

The fractal-fractional integrals with power-law, exponential decay, and Mittag-Leffler kernel are as follows:

$$
\begin{aligned}
& { }_{0}^{F F P} J_{t}^{\alpha, \beta} f(t)=\frac{1}{\Gamma(\alpha)} \int_{0}^{t}(t-\tau)^{\alpha-1} \tau^{1-\beta} f(\tau) d \tau, \\
& { }_{0}^{F F E} J_{t}^{\alpha, \beta} f(t)=\frac{1-\alpha}{M(\alpha)} t^{1-\beta} f(t)+\frac{\alpha}{M(\alpha)} \int_{0}^{t} \tau^{1-\beta} f(\tau) d \tau, \\
& { }_{0}^{F F M} J_{t}^{\alpha, \beta} f(t)=\frac{1-\alpha}{A B(\alpha)} t^{1-\beta} f(t)+\frac{\alpha}{A B(\alpha) \Gamma(\alpha)} \int_{0}^{t}(t-\tau)^{\alpha-1} \tau^{1-\beta} f(\tau) d \tau .
\end{aligned}
$$

\section{Box-Jenkin's model development}

Autoregressive integrated moving average (ARIMA) approach suggested by Box and Jenkins is one of the most powerful techniques used in time series analysis. The ARIMA model is composed of three parts. First, the autoregressive part is a linear regression which has a relation between past values and future values of data series; second, the integrated part expresses how many times the data series has to be differenced to obtain a stationary series; and the last one is the moving average part which has a relation between past forecast errors and future values of data series [14]. These processes can be presented by the models $\operatorname{AR}(p), \operatorname{MA}(q), \operatorname{ARMA}(p, q)$, and $\operatorname{ARIMA}(p, d, q)$. We should decide which model we will choose for our data series. To do this, partial autocorrelation (PACF) and the autocorrelation (ACF) are helpful to obtain parameters for the AR model and the MA model, respectively.

Figures 1 and 2 depict graphs of autocorrelation functions for the infected and deaths in Africa and Europe.

Now we introduce these models. Let $Y_{t}$ be the value of the time series at time $t$. Time series as a $p$-order autoregressive process is as follows:

$$
Y_{t}=\delta+\varphi_{1} Y_{t-1}+\varphi_{2} Y_{t-2}+\cdots+\varphi_{p} Y_{t-p}+\varepsilon_{t},
$$

which is shown as $\operatorname{AR}(p)$. Here, $\delta$ and $\varepsilon_{t}$ describe constant and error terms, respectively. Time series as a $q$ th degree of moving average process is given by

$$
Y_{t}=\mu+\varepsilon_{t}+\theta_{1} \varepsilon_{t-1}+\theta_{2} \varepsilon_{t-2}+\cdots+\theta_{q} \varepsilon_{t-q}
$$



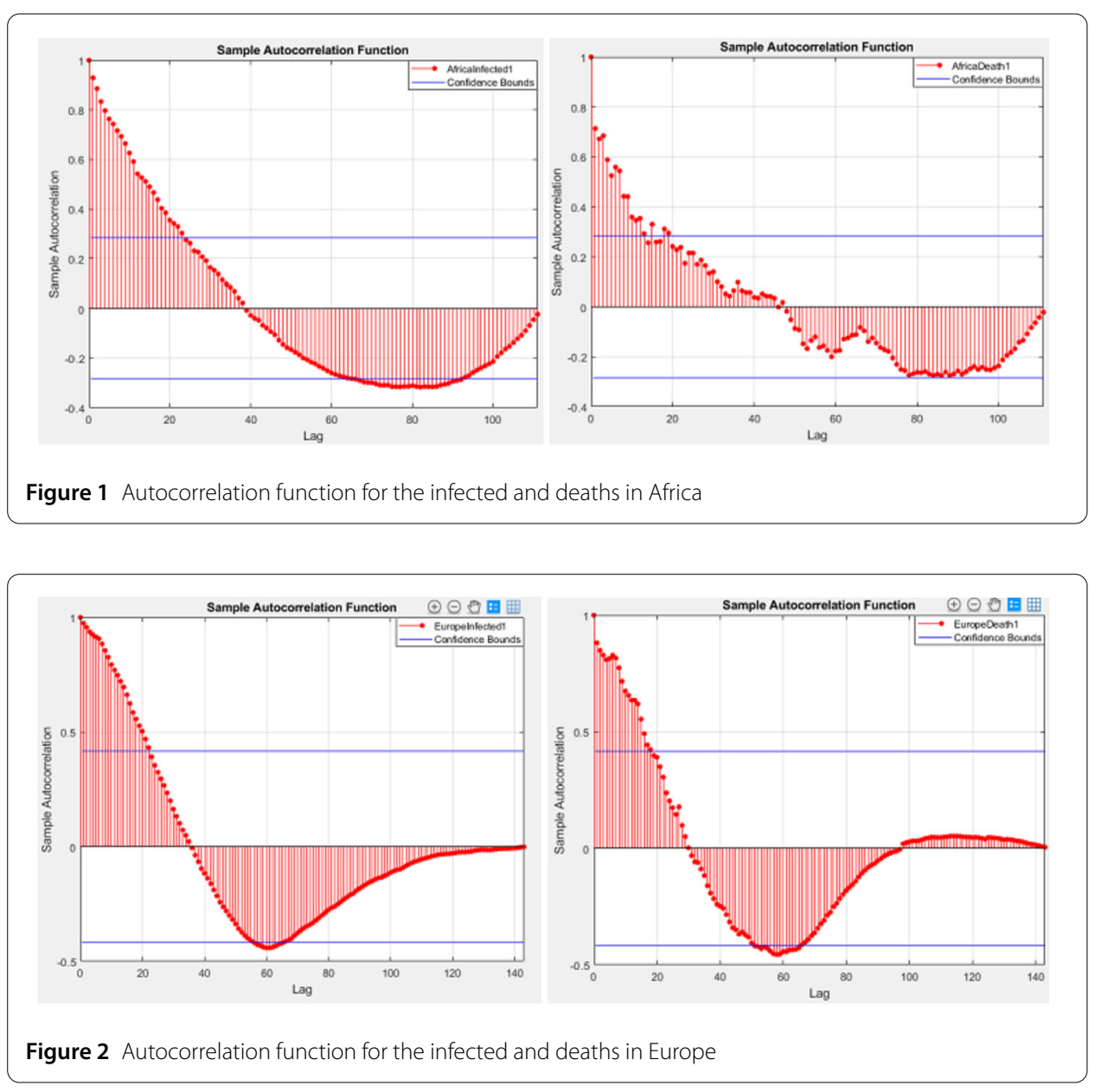

which is shown as $\operatorname{MA}(q)$. The $\operatorname{ARMA}(p, q)$ expression is obtained as a combination of $\operatorname{AR}(p)$ and $\operatorname{MA}(q)$ equations:

$$
Y_{t}=\delta+\varphi_{1} Y_{t-1}+\varphi_{2} Y_{t-2}+\cdots+\varphi_{p} Y_{t-p}+\varepsilon_{t}+\theta_{1} \varepsilon_{t-1}+\theta_{2} \varepsilon_{t-2}+\cdots+\theta_{q} \varepsilon_{t-q}
$$

When the time series is not stationary, we take the difference $d$ times to make it stationary. The $\operatorname{ARIMA}(p, q)$ model is given by

$$
\left(1-\varphi_{1} l-\varphi_{2} l^{2}-\cdots-\varphi_{p} l^{p}\right) \Delta^{d} Y_{t}=\delta+\varepsilon_{t}+\theta_{1} \varepsilon_{t-1}+\theta_{2} \varepsilon_{t-2}+\cdots+\theta_{q} \varepsilon_{t-q}
$$

In the ARIMA technique, the model performance can be measured by using some criteria, for instance, Akaike information criteria(AIC), Bayesian information criteria(BIC). Here, we benefit from the Akaike information criteria given as follows:

$$
\begin{aligned}
& A I C=-2 \log (l)+2 k, \\
& B I C=-2 \log (l)+k \ln n,
\end{aligned}
$$

where $l$ states likelihood of the data, $n$ is the number of data points, and $k$ also defines the intercept of the ARIMA model. The numerical simulation are depicted in Figs. 3, 4, 5 and 6. 
According to data series for the infected in Africa, we use the $\operatorname{ARIMA}(2,1,0)$ model which is given by

$$
\left(1-\varphi_{1} l-\varphi_{2} l^{2}\right)(1-l) Y_{t}=c+\varepsilon_{t}
$$

Here,

$$
\begin{aligned}
& A I C=1670.1734, \\
& B I C=1680.9388 .
\end{aligned}
$$

In Table 1, we give parameter estimation for infections in Africa.

According to data series for deaths in Africa, we use the $\operatorname{AR}(1)$ model which is given by

$$
\left(1-\varphi_{1} l\right) Y_{t}=c+\varepsilon_{t}
$$

Here,

$$
\begin{aligned}
& A I C=1056.6482, \\
& B I C=1064.7768 .
\end{aligned}
$$

In Table 2, we give parameter estimation for deaths in Africa.

Table 1 Model estimation for infections in Africa

\begin{tabular}{llll}
\hline Parameter & Value & Standard error & TStatistic \\
\hline Constant & 89.2032 & 56.6511 & 1.5746 \\
AR $\{1\}$ & -0.44796 & 0.099221 & -4.5147 \\
AR $\{2\}$ & -0.17789 & 0.068294 & -2.6047 \\
Variance & $168,446.2911$ & $12,738.3089$ & 13.2236 \\
\hline
\end{tabular}

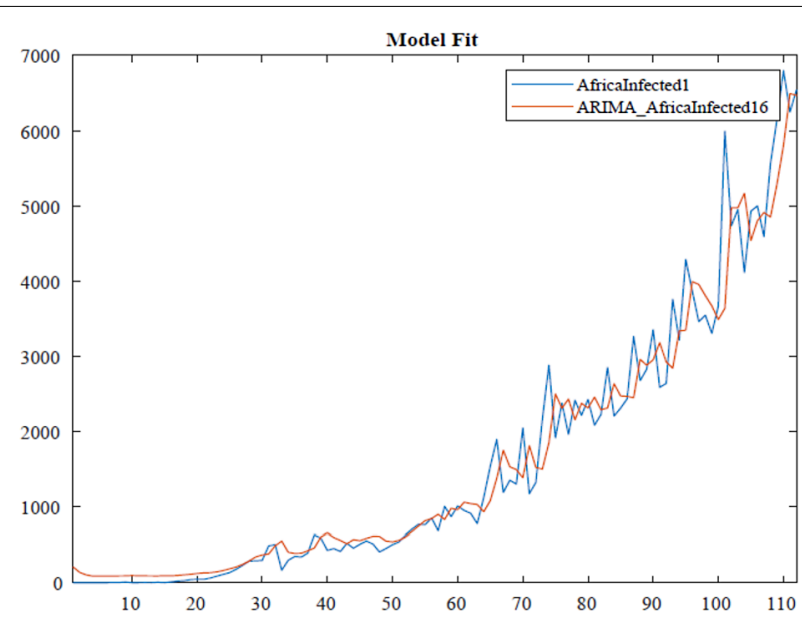

Figure 3 ARIMA model for the infected in Africa 
Table 2 Model estimation for deaths in Africa

\begin{tabular}{llll}
\hline Parameter & Value & Standard error & TStatistic \\
\hline Constant & 12.581 & 6.0023 & 2.096 \\
AR\{1\} & 0.75094 & 0.082701 & 9.0802 \\
Variance & 694.3043 & 92.168 & 7.533 \\
\hline
\end{tabular}

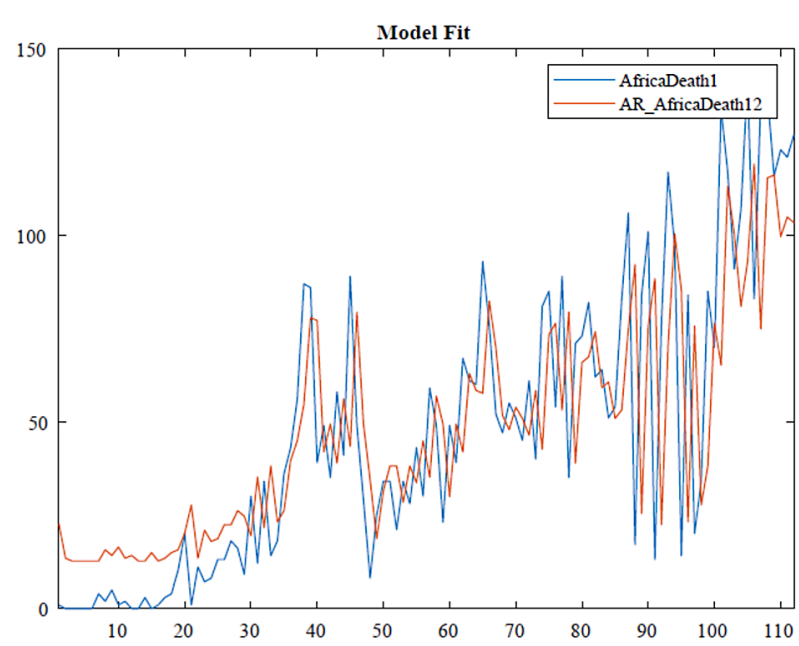

Figure 4 AR model for deaths in Africa

Table 3 Model estimation for the infected in Europe

\begin{tabular}{llll}
\hline Parameter & Value & Standard error & TStatistic \\
\hline Constant & 83.7826 & 118.7108 & 0.70577 \\
AR\{1\} & 0.3216 & 0.59303 & 0.5423 \\
AR $\{2\}$ & 0.035772 & 0.16277 & 0.21977 \\
MA $\{1\}$ & -0.53222 & 0.58359 & -0.91197 \\
Variance & $7,214,609.9182$ & $569,786.6944$ & 12.6619 \\
\hline
\end{tabular}

According to data series for the infected in Europe, we use the $\operatorname{ARIMA}(2,1,1)$ model which is given by

$$
\left(1-\varphi_{1} l-\varphi_{2} l^{2}\right)(1-l) Y_{t}=c+\left(1+\theta_{1} l\right) \varepsilon_{t}
$$

Here,

$$
\begin{aligned}
& A I C=2690.5358, \\
& B I C=2705.2796 .
\end{aligned}
$$

In Table 3, we give parameter estimation for the infected in Europe.

According to data series for deaths in Europe, we use the AR(1) model which is given by

$$
\left(1-\varphi_{1} l\right) Y_{t}=c+\varepsilon_{t} .
$$




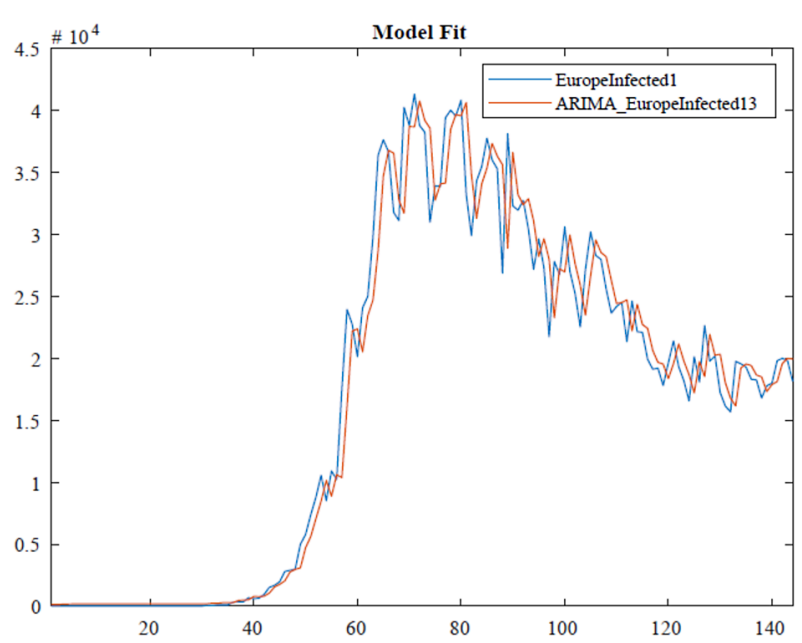

Figure 5 ARIMA model for the infected in Europe

Table 4 Model estimation for deaths in Europe

\begin{tabular}{llll}
\hline Parameter & Value & Standard error & TStatistic \\
\hline Constant & 151.4852 & 163.967 & 0.92388 \\
AR\{1\} & 0.8865 & 0.041096 & 21.5714 \\
Variance & $460,062.22$ & $27,485.093$ & 16.7386 \\
\hline
\end{tabular}

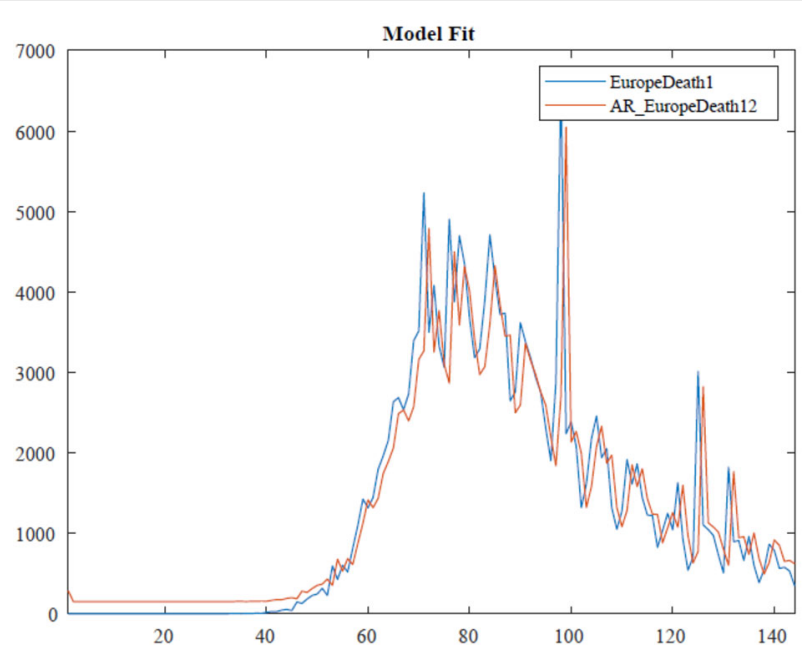

Figure 6 AR model for deaths in Europe

Here,

$$
\begin{aligned}
& A I C=1670.1734, \\
& B I C=1680.9388 .
\end{aligned}
$$

In Table 4, we give parameter estimation for deaths in Europe. 


\section{Brown's exponential smoothing method}

Brown's linear exponential smoothing is one type of double exponential smoothing based on two different smoothed series. The formula is composed of an extrapolation of a line through the two centers. The Brown exponential smoothing method is helpful to model the time series having trend but no seasonality.

For non-adaptive Brown exponential smoothing, the procedure can be described as follows.

Firstly, we start with the following initialization:

1) $S_{0}=u_{0}$,

2) $T_{0}=u_{0}$

3) $a_{0}=2 S_{0}-T_{0}$,

4) $F_{1}=a_{0}+b_{0}$.

Then we have the following calculations:

1) $S_{t}=\alpha u_{t}+(1-\alpha) S_{t-1}$,

2) $T_{t}=\alpha S_{t}+(1-\alpha) T_{t-1}$,

3) $a_{t}=2 S_{t}+T_{t} 63$,

4) $\alpha\left(S_{t}-T_{t}\right)=(1-\alpha) b_{t}$,

5) $F_{t+1}=a_{t}+b_{t}$,

where $0<\alpha<1$ is the smoothing factor. $S_{t}$ and $T_{t}$ are the simply smoothed value and doubly smoothed value for the $(t+1)$ th time period, respectively. Also $a_{t}$ and $b_{t}$ describe the intercept and the slope, respectively.

In Figs. 7, 8, 9, and 10, we present the simulation for smoothing method for the infected and deaths in Africa and Europe where the smoothing factor was chosen as $\alpha=0.99$.

\section{Future prediction of daily new numbers of the infected and deaths: Africa and Europe}

With the collected data using some statistical formula, it is possible to predict what will possibly happen in the near future. Having in mind what could possibly happen, several measures could be taken to avoid the worst case scenario. In this section, with the data collected for 101 countries from Africa (47) and Europe (54), we aim at presenting possible

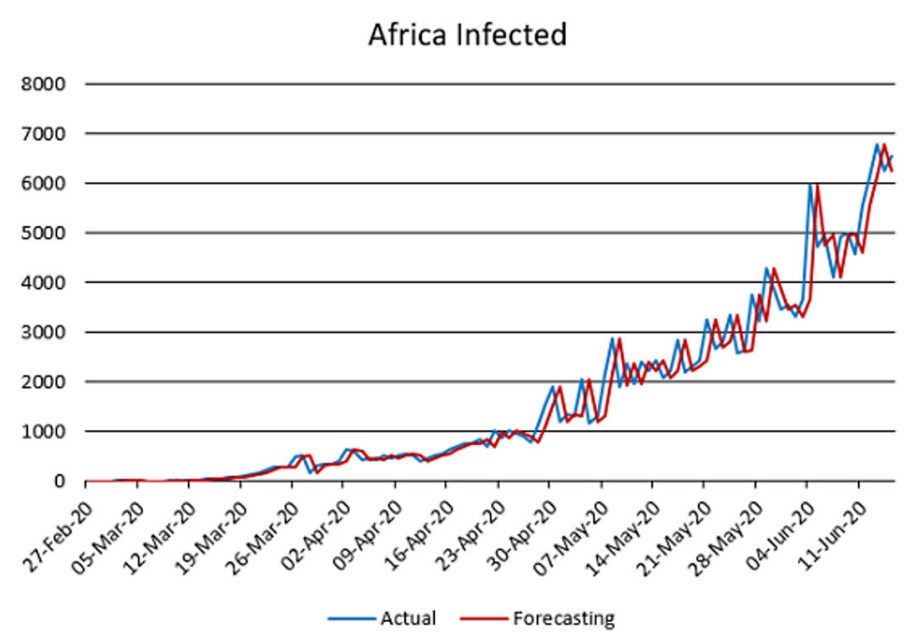

Figure 7 Exponential smoothing for the infected in Africa 

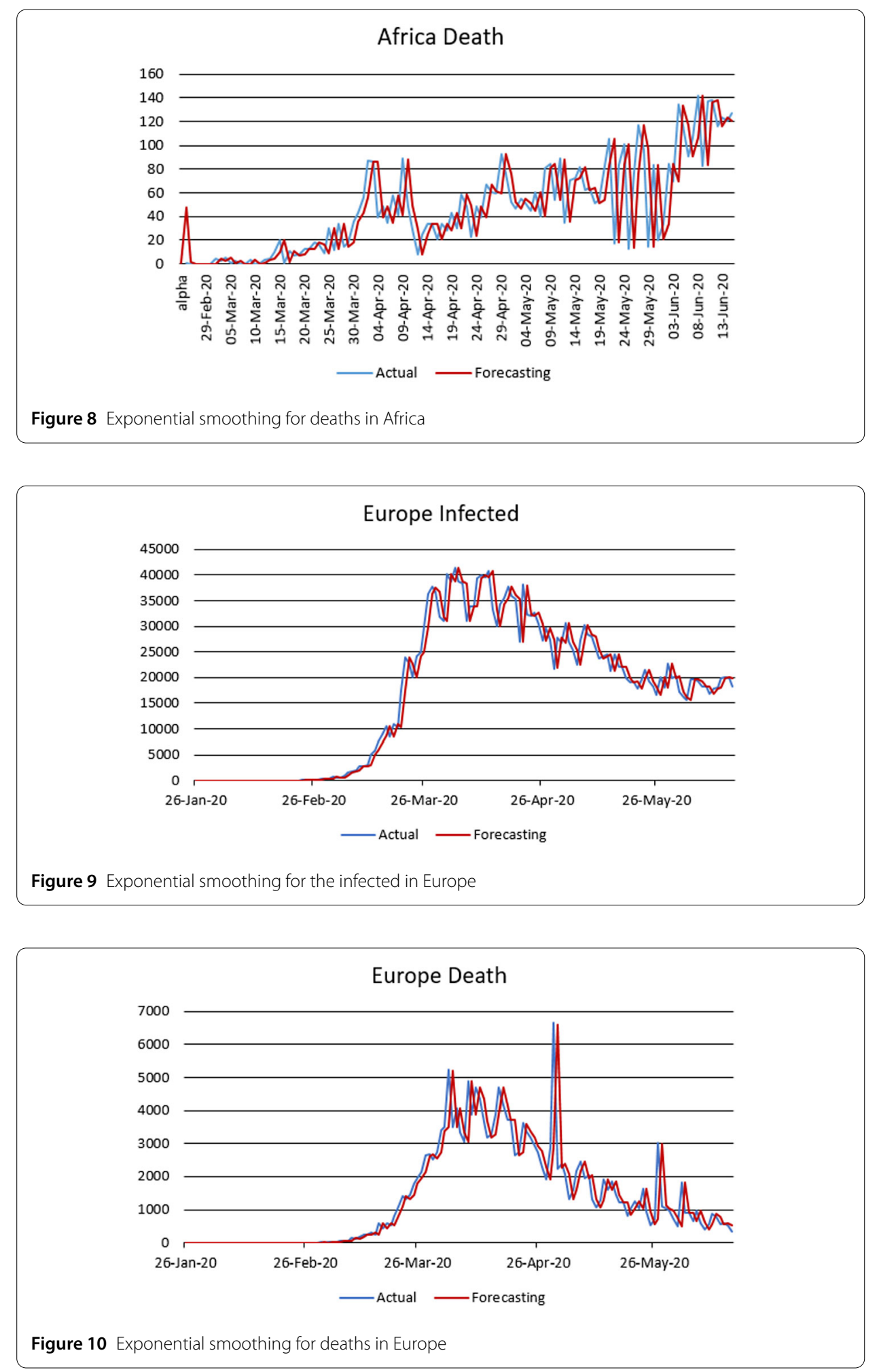

scenarios or events that could be observed in the near future, the daily numbers of deaths and infections. Numerical simulation are presented in Figs. 11, 12, 13 and 14.

In Figs. 15, 16, 17, and 18, we present fitting with smoothing spline for the infected and deaths in Africa and Europe. 

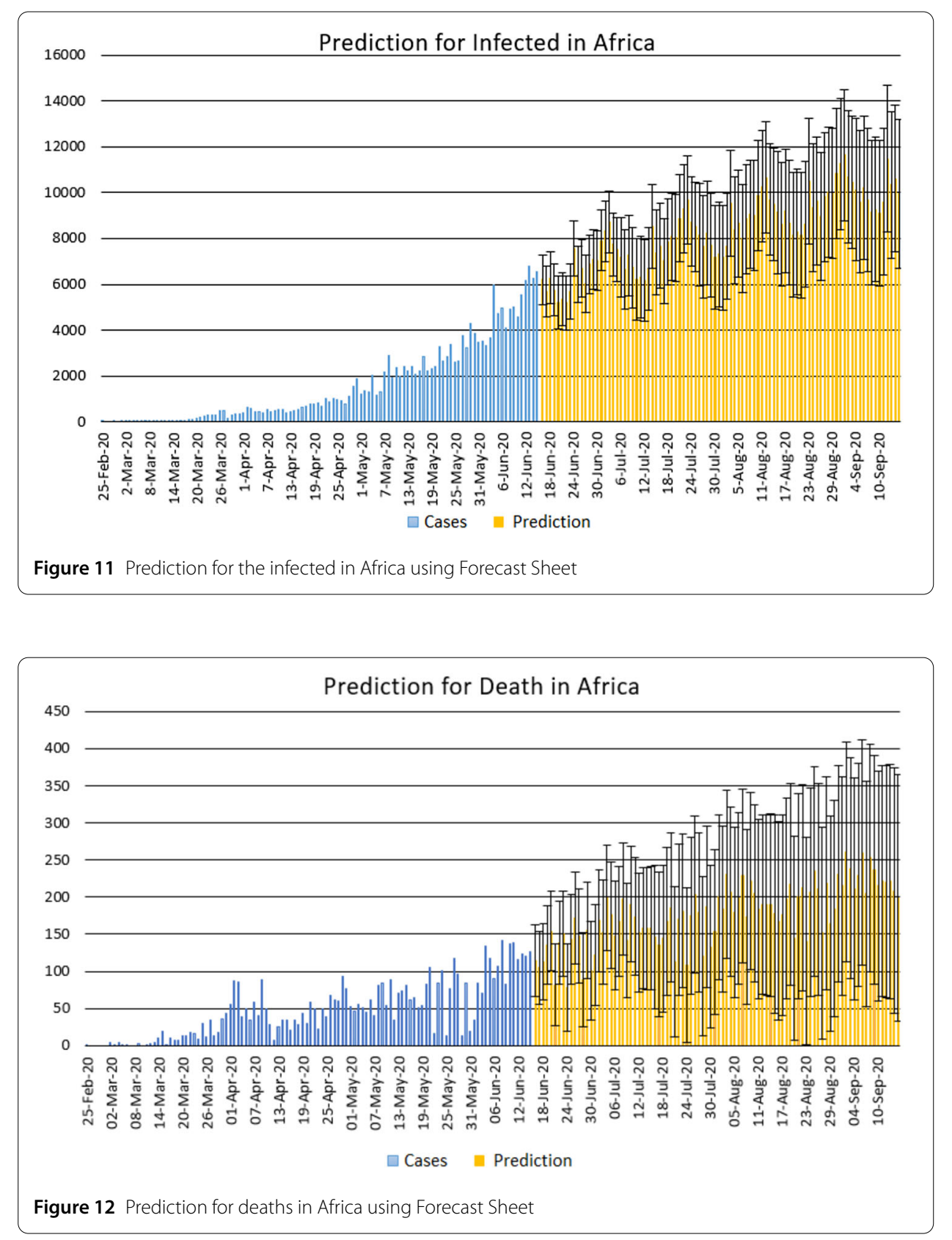

\section{An analysis of COVID-19 spread based on fractal interpolation and fractal dimension}

In this section, we present some information about fractal dimension, interpolation, and blancmange curve.

\subsection{Fractal dimension}

Fractal dimensions enable us to compare fractals. Fractal dimensions are important because they can be defined in connection with real-world data, and they can be measured approximately by means of experiments. These numbers allow us to compare sets in the real world with the laboratory fractals. 

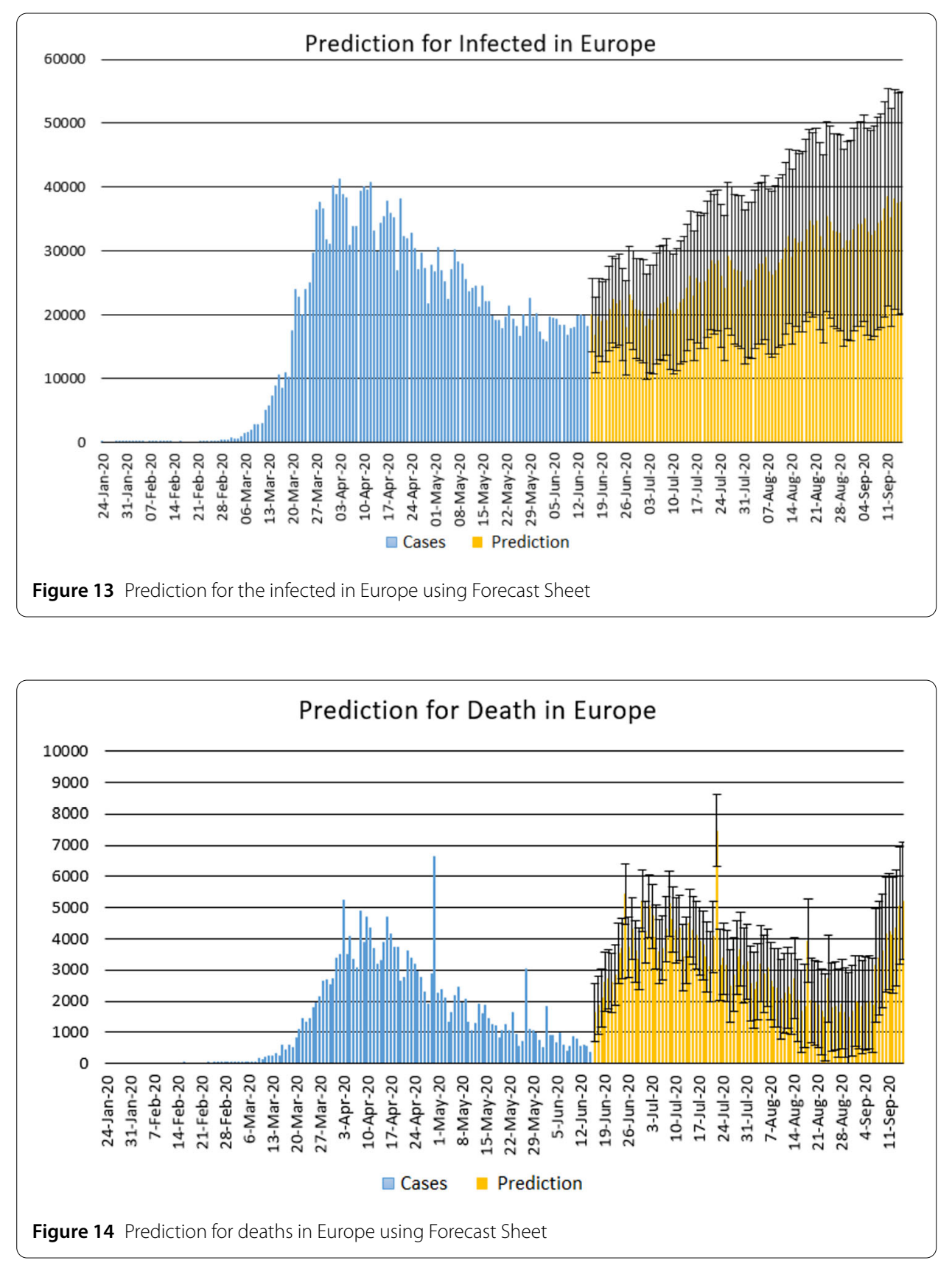

Theorem (The box counting theorem) Let $N_{n}(A)$ be the number of boxes of side length $\left(1 / 2^{n}\right)$. Then the fractal dimension $D$ of $A$ is given as [15]

$$
D=\lim _{n \rightarrow \infty}\left\{\frac{\ln \left[N_{n}(A)\right]}{\ln \left(2^{n}\right)}\right\}
$$

\subsection{Fractal interpolation}

Euclidean geometry and calculus enable us to model using some lines and curves, the shapes that we encounter in the nature $[15,16]$. In this section, we present an interpolation function which interpolates the data. 


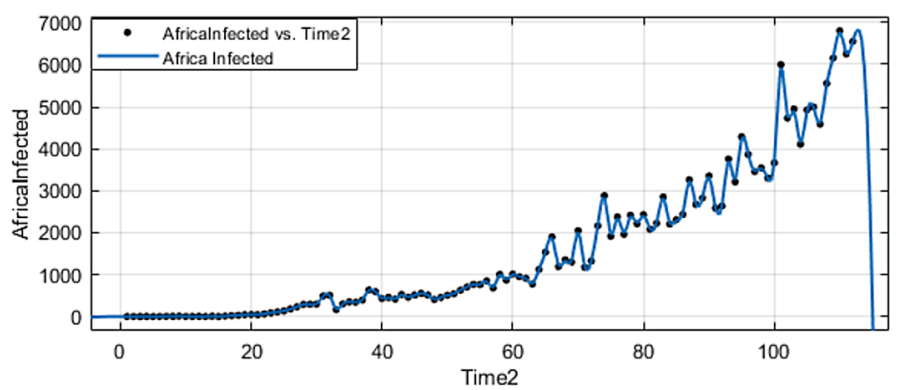

Figure 15 Fitting for the infected in Africa

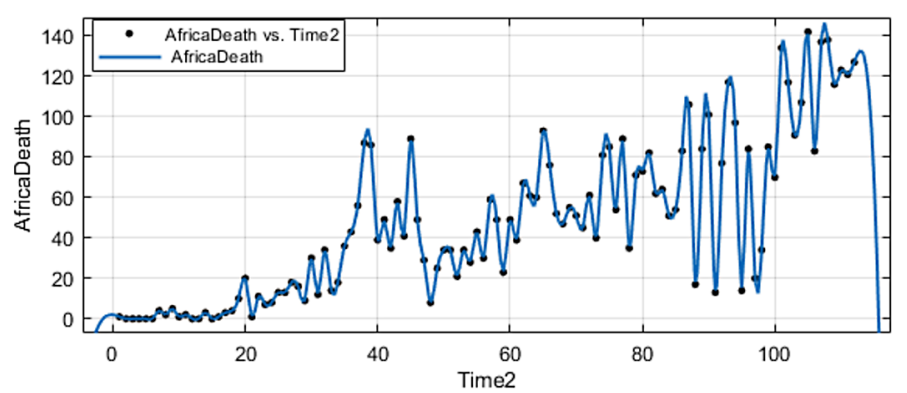

Figure 16 Fitting for deaths in Africa

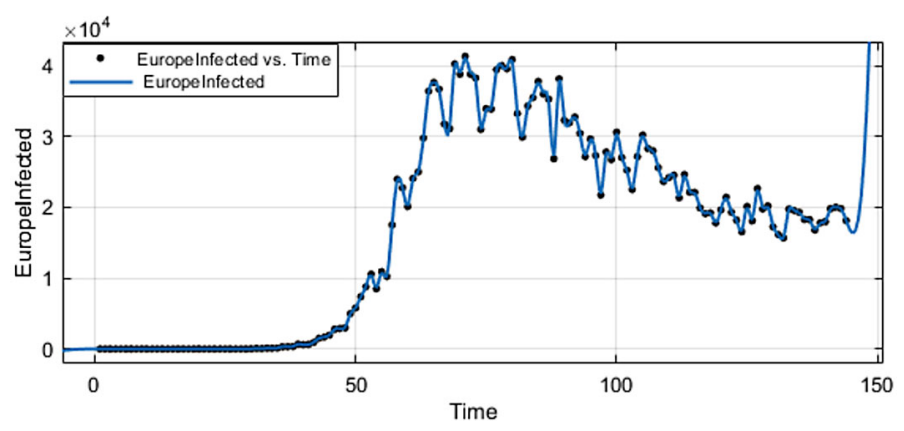

Figure 17 Fitting for the infected in Europe

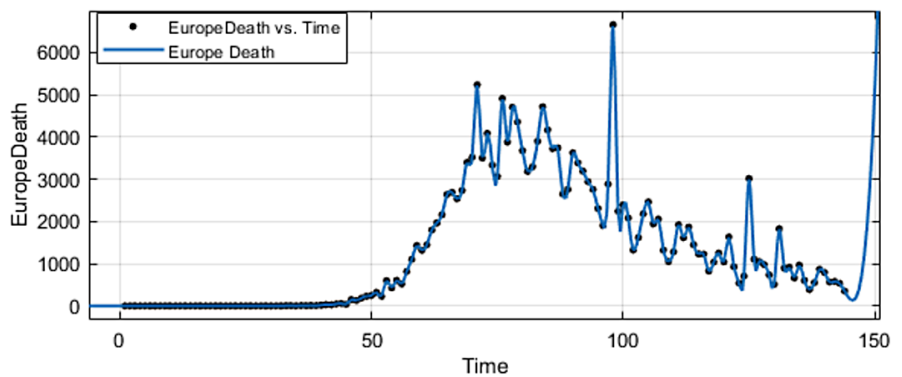

Figure 18 Fitting for deaths in Europe 
Definition 2 An interpolation function $f:\left[x_{0}, x_{N}\right] \rightarrow \mathbb{R}$ corresponding to the set of data $\left\{\left(x_{i}, F_{i}\right) \in \mathbb{R}^{2}: i=0,1,2, \ldots, N\right\}[15]$

$$
f\left(x_{i}\right)=F_{i} \quad \text { for } i=1,2, \ldots, N
$$

where $x_{0}<x_{1}<x_{2} \cdots<x_{N}$.

Let $f:\left[x_{0}, x_{N}\right] \rightarrow \mathbb{R}$ denote the unique continuous function which is called a piecewise linear interpolation function. Also this function is linear on each of the subintervals $\left[x_{i-1}, x_{i}\right]$, and it is represented by

$$
f(x)=F_{i-1}+\frac{\left(x-x_{i-1}\right)}{\left(x_{i}-x_{i-1}\right)}\left(F_{i}-F_{i-1}\right) \quad \text { for } x \in\left[x_{i-1}, x_{i}\right], i=1,2, \ldots, N .
$$

We have the following transformation, which is iterated:

$$
f_{n}\left(\begin{array}{l}
x \\
y
\end{array}\right)=\left(\begin{array}{cc}
t_{n} & 0 \\
u_{n} & y_{n}
\end{array}\right)\left(\begin{array}{l}
x \\
y
\end{array}\right)+\left(\begin{array}{l}
v_{n} \\
w_{n}
\end{array}\right) .
$$

When solving this system for $t_{n}, u_{n}, v_{n}$, and $w_{n}$ in terms of the data and $y_{n}$, we obtain the following:

$$
\begin{aligned}
t_{n} & =\frac{x_{n}-x_{n-1}}{x_{N}-x_{0}}, \\
u_{n} & =\frac{F_{n}-F_{n-1}}{x_{N}-x_{0}}-y_{n} \frac{F_{n}-F_{0}}{x_{N}-x_{0}}, \\
v_{n} & =\frac{x_{N} x_{n-1}-x_{0} x_{n}}{x_{N}-x_{0}}, \\
w_{n} & =\frac{x_{N} F_{n-1}-x_{0} F_{n}}{x_{N}-x_{0}}-y_{n} \frac{x_{N} F_{0}-x_{0} F_{n}}{x_{N}-x_{0}},
\end{aligned}
$$

where $0 \leq y_{n}<1$ is called the scaling factor [15].

\subsection{Blancmange curve}

The blancmange function can be given as an example of fractal interpolation function, and this function is defined by

$$
\sum_{n=0}^{\infty} \frac{S\left(2^{n} x\right)}{2^{n}}, \quad x \in[0,1],
$$

where $S(x)=\min _{m \in \mathbb{Z}}|x-m|, x \in \mathbb{R}$.

However, many problems cannot be depicted when $c=2$ [16]. Then we discuss the limitations of this blancmange; for example, $t$ can only go from 0 to 1 , the periodic parameter is 2 . Therefore, we change 2 to $c$, where $c$ is a real number from 1 to $a$. Therefore, in this section, we extend the blancmange function to a large interval also with any given periodic parameter. So, we have the following formula:

$$
\sum_{n=0}^{\infty} \frac{S\left(c^{n} x\right)}{c^{n}}, \quad x \in[0, a],
$$



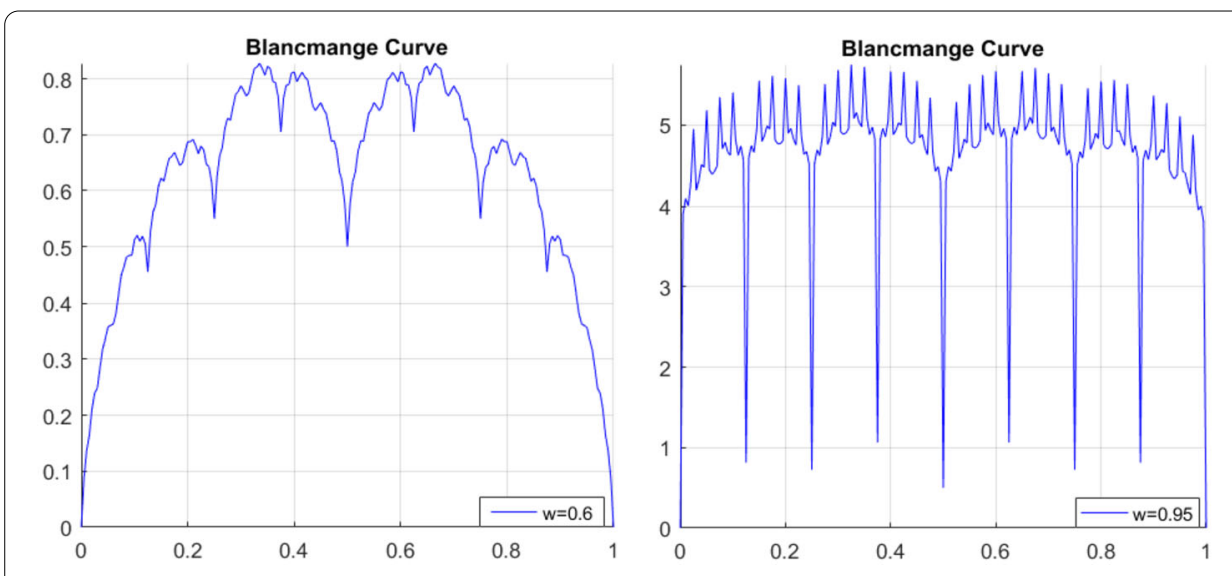

Figure 19 Blancmange function $c=2$
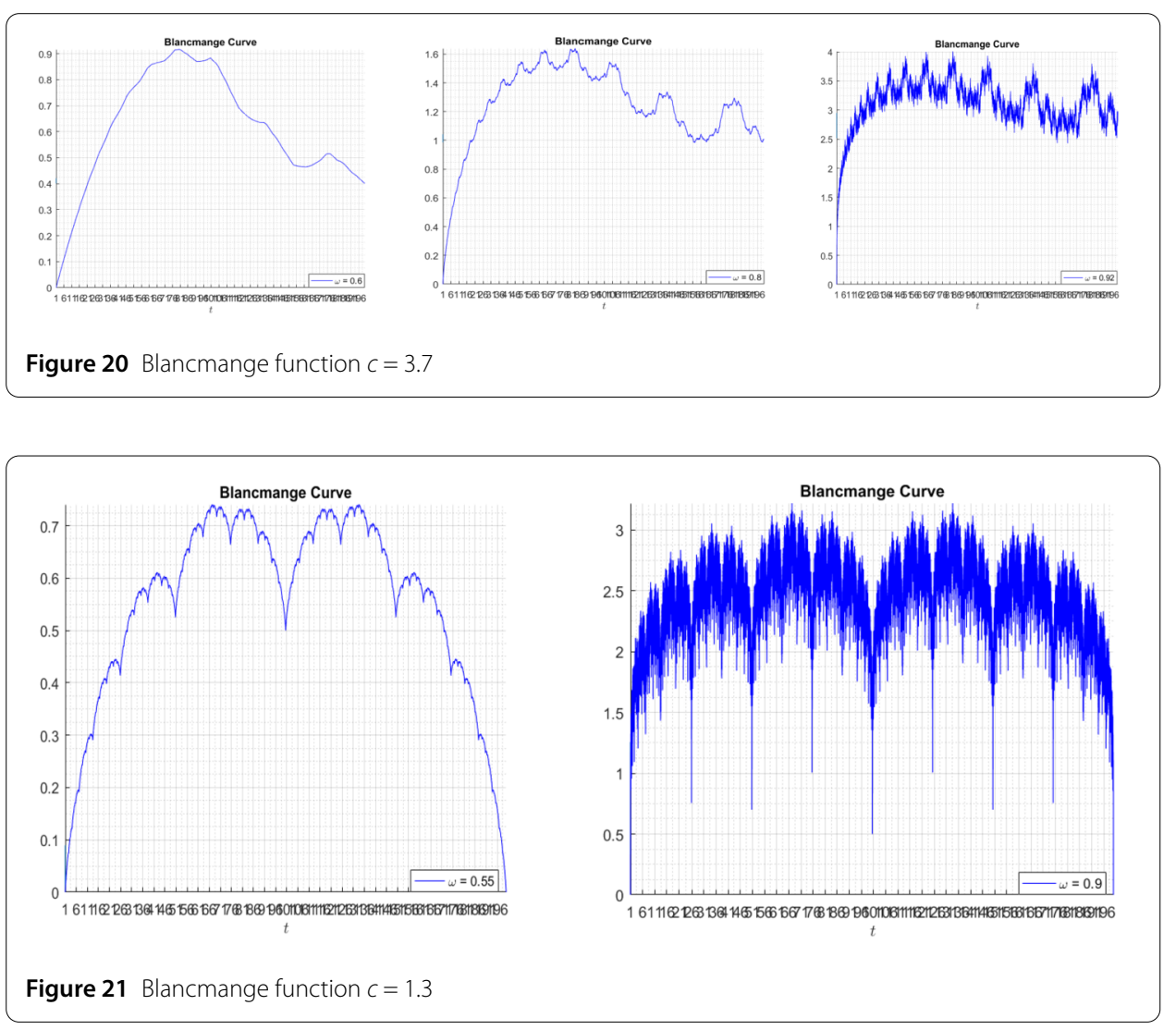

where $c$ is the real number. We now present the extended blancmange function for different periodic parameters and different $w$.

The simulation are presented in Figs. 19, 20, 21, and 18.

\section{Mathematical model for COVID-19 outbreak}

We consider the following mathematical model of COVID-19 spread:

$$
\dot{S}=\Lambda-\left\{\delta(t)\left(\alpha I+w\left(\beta I_{D}+\gamma I_{A}+\delta_{1} I_{R}+\delta_{2} I_{T}\right)+\gamma_{1}+\mu_{1}\right)\right\} S,
$$




$$
\begin{aligned}
& \dot{I}=\delta(t)\left(\alpha I+w\left(\beta I_{D}+\gamma I_{A}+\delta_{1} I_{R}+\delta_{2} I_{T}\right)\right) S-\left(\varepsilon+\xi+\lambda+\mu_{1}\right) I, \\
& \dot{I_{A}}=\xi I-\left(\theta+\mu+\chi+\mu_{1}\right) I_{A}, \\
& \dot{I_{D}}=\varepsilon I-\left(\eta+\varphi+\mu_{1}\right) I_{D}, \\
& \dot{I_{R}}=\eta I_{D}+\theta I_{A}-\left(v+\xi+\mu_{1}\right) I_{R}, \\
& \dot{I_{T}}=\mu I_{A}+v I_{R}-\left(\sigma+\tau+\mu_{1}\right) I_{T}, \\
& \dot{R}=\lambda I+\varphi I_{D}+\chi I_{A}+\xi I_{R}+\sigma I_{T}-\left(\Phi+\mu_{1}\right) R, \\
& \dot{D}=\tau I_{T}, \\
& \dot{V}=\gamma_{1} S+\Phi R-\mu_{1} V .
\end{aligned}
$$

The above model was suggested by Atangana and Seda, the model has a deterministic character. In this section, we convert the model to a stochastic one by introducing the effect of environmental white noise. To achieve this, we reformulate the model by adding the nonlinear perturbation into each equation of the system. The perturbation may depend on square of the classes $S, I, I_{A}, I_{D}, I_{R}, I_{T}, R, D$, and $V$ respectively. Here, we perturb only the rate of each class. However, for the vaccine class, it will be perturbed by a natural death rate.

For the class $S(t): \quad-\gamma_{1} \rightarrow-\gamma_{1}+\left(\Pi_{11} S+\Pi_{12}\right) \dot{B}_{1}(t)$,

For the class $I(t): \quad-\lambda \rightarrow-\lambda+\left(\Pi_{21} I+\Pi_{22}\right) \dot{B}_{2}(t)$,

For the class $I_{A}(t): \quad-\theta \rightarrow-\theta+\left(\Pi_{31} I_{A}+\Pi_{32}\right) B_{3}(t)$,

For the class $I_{D}(t): \quad-\eta \rightarrow-\eta+\left(\Pi_{41} I_{D}+\Pi_{42}\right) B_{4}(t)$,

For the class $I_{R}(t): \quad-v \rightarrow-v+\left(\Pi_{51} I_{R}+\Pi_{52}\right) \dot{B}_{5}(t)$,

For the class $I_{T}(t): \quad-\sigma \rightarrow-\sigma+\left(\Pi_{61} I_{T}+\Pi_{62}\right) \dot{B}_{6}(t)$,

For the class $R(t): \quad-\Phi \rightarrow-\Phi+\left(\Pi_{71} R+\Pi_{72}\right) \dot{B}_{7}(t)$,

For the class $D(t): \quad \tau \rightarrow \tau$ no change,

For the class $V(t): \quad-\mu_{1} \rightarrow-\mu_{1}+\left(\Pi_{81} V+\Pi_{82}\right) \dot{B}_{8}(t)$.

Therefore, the associated stochastic model is given as follows:

$$
\begin{aligned}
d S= & {\left[\Lambda-\left\{\delta(t)\left(\alpha I+w\left(\beta I_{D}+\gamma I_{A}+\delta_{1} I_{R}+\delta_{2} I_{T}\right)+\gamma_{1}+\mu_{1}\right)\right\} S\right] d t } \\
& +\left(\Pi_{11} S+\Pi_{12}\right) S d B_{1}(t), \\
d I= & {\left[\delta(t)\left(\alpha I+w\left(\beta I_{D}+\gamma I_{A}+\delta_{1} I_{R}+\delta_{2} I_{T}\right)\right) S-\left(\varepsilon+\xi+\lambda+\mu_{1}\right) I\right] d t } \\
& +\left(\Pi_{21} I+\Pi_{22}\right) I d B_{2}(t), \\
d I_{A}= & {\left[\xi I-\left(\theta+\mu+\chi+\mu_{1}\right) I_{A}\right] d t+\left(\Pi_{31} I_{A}+\Pi_{32}\right) I_{A} d B_{3}(t), }
\end{aligned}
$$




$$
\begin{aligned}
& d I_{D}=\left[\varepsilon I-\left(\eta+\varphi+\mu_{1}\right) I_{D}\right] d t+\left(\Pi_{41} I_{D}+\Pi_{42}\right) I_{D} d B_{4}(t), \\
& d I_{R}=\left[\eta I_{D}+\theta I_{A}-\left(\nu+\xi+\mu_{1}\right) I_{R}\right] d t+\left(\Pi_{51} I_{R}+\Pi_{52}\right) I_{D} d B_{5}(t), \\
& d I_{T}=\left[\mu I_{A}+v I_{R}-\left(\sigma+\tau+\mu_{1}\right) I_{T}\right] d t+\left(\Pi_{61} I_{T}+\Pi_{62}\right) I_{T} d B_{6}(t), \\
& d R=\left[\lambda I+\varphi I_{D}+\chi I_{A}+\xi I_{R}+\sigma I_{T}-\left(\Phi+\mu_{1}\right) R\right] d t+\left(\Pi_{71} R+\Pi_{72}\right) R d B_{7}(t), \\
& d V=\left[\gamma_{1} S+\Phi R-\mu_{1} V\right] d t++\left(\Pi_{71} V+\Pi_{72}\right) V d B_{8}(t) .
\end{aligned}
$$

In this conversion, the function $B_{i}(t)$ represents the standard Brownian motions valid within the set of probability $\left(\Omega, A,\left\{A_{t}\right\}_{t \geq 0}, P\right)$, where $\left\{A_{t}\right\}_{t \geq 0}$ is filtration valid under the condition described in [17]. Here, $\Pi_{i, j \in[1,2,3,4,5,6,7,8]}$ are positive and are the intensities of the environmental random disturbance.

\subsection{Existence and uniqueness}

In this subsection, we present the existence and uniqueness of the system solutions of the stochastic model. To achieve the existence and uniqueness, we convert the system into Volterra type. But first we do the following for simplicity:

$$
\begin{aligned}
& d S=F_{1}\left(t, S, I, I_{A}, I_{D}, I_{R}, I_{T}, R, V\right) d t+G_{1}(t, S) d B_{1}(t) \\
& d I=F_{2}\left(t, S, I, I_{A}, I_{D}, I_{R}, I_{T}, R, V\right) d t+G_{2}(t, I) d B_{2}(t), \\
& d I_{A}=F_{3}\left(t, I, I_{A}\right) d t+G_{3}\left(t, I_{A}\right) d B_{3}(t) \\
& d I_{D}=F_{4}\left(t, I, I_{D},\right) d t+G_{4}\left(t, I_{D}\right) d B_{4}(t) \\
& d I_{R}=F_{5}\left(t, I_{A}, I_{D}, I_{R}\right) d t+G_{5}\left(t, I_{R}\right) d B_{5}(t) \\
& d I_{T}=F_{6}\left(t, I_{A}, I_{R}, I_{T}\right) d t+G_{6}\left(t, I_{T}\right) d B_{6}(t) \\
& d R=F_{7}\left(t, I, I_{A}, I_{D}, I_{R}, I_{T}, R\right) d t+G_{7}(t, R) d B_{7}(t) \\
& d V=F_{8}(t, S, R, V) d t+G_{8}(t, V) d B_{8}(t) .
\end{aligned}
$$

Therefore, converting to Volterra, we get

$$
\begin{aligned}
& S(t)=S(0)+\int_{0}^{t} F_{1}\left(\tau, S, I, I_{A}, I_{D}, I_{R}, I_{T}, R, V\right) d \tau+\int_{0}^{t} G_{1}(\tau, S) d B_{1}(\tau) \\
& I(t)=I(0)+\int_{0}^{t} F_{2}\left(\tau, S, I, I_{A}, I_{D}, I_{R}, I_{T}, R, V\right) d \tau+\int_{0}^{t} G_{2}(\tau, I) d B_{2}(\tau) \\
& I_{A}(t)=I_{A}(0)+\int_{0}^{t} F_{3}\left(\tau, I, I_{A}\right) d \tau+\int_{0}^{t} G_{3}\left(\tau, I_{A}\right) d B_{3}(\tau) \\
& I_{D}(t)=I_{D}(0)+\int_{0}^{t} F_{4}\left(\tau, I, I_{D}\right) d \tau+\int_{0}^{t} G_{4}\left(\tau, I_{D}\right) d B_{4}(\tau) \\
& I_{R}(t)=I_{R}(0)+\int_{0}^{t} F_{5}\left(\tau, I_{A}, I_{D}, I_{R}\right) d \tau+\int_{0}^{t} G_{5}\left(\tau, I_{R}\right) d B_{5}(\tau) \\
& I_{T}(t)=I_{T}(0)+\int_{0}^{t} F_{6}\left(\tau, I_{A}, I_{R}, I_{T}\right) d \tau+\int_{0}^{t} G_{6}\left(\tau, I_{T}\right) d B_{6}(\tau)
\end{aligned}
$$




$$
\begin{aligned}
& R(t)=R(0)+\int_{0}^{t} F_{7}\left(\tau, I, I_{A}, I_{D}, I_{R}, I_{T}, R\right) d \tau+\int_{0}^{t} G_{7}(\tau, R) d B_{7}(\tau), \\
& V(t)=V(0)+\int_{0}^{t} F_{8}(\tau, S, R, V) d \tau+\int_{0}^{t} G_{8}(\tau, S) d B_{8}(\tau) .
\end{aligned}
$$

We present the existence and uniqueness of the stochastic system of COVID-19 model. This will be achieved via the following theorem.

Theorem Assume that there exist positive constants $K_{i}, \bar{K}_{i}$ such that

$$
\begin{aligned}
& \left|F_{i}(x, t)-F_{i}\left(x_{i}, t\right)\right|^{2}<K_{i}\left|x-x_{i}\right|^{2}, \\
& \left|G_{i}(x, t)-G_{i}\left(x_{i}, t\right)\right|^{2}<\bar{K}_{i}\left|x-x_{i}\right|^{2}
\end{aligned}
$$

(ii) $\forall(x, t) \in R^{8} \times[0, T]$

$$
\left|F_{i}(x, t)\right|^{2},\left|G_{i}(x, t)\right|^{2}<K\left(1+|x|^{2}\right) .
$$

Then there exists a unique solution $X(t) \in R^{8}$ for our model and it belongs to $M^{2}([0, T]$, $\left.R^{8}\right)$.

The proof can be found in [17], but we have to verify (i) and (ii) for our system. Without loss of generality, we start our investigation with functions $F_{1}\left(t, S, I, I_{A}, I_{D}, I_{R}, I_{T}, R, V\right)$ and $G_{1}(t, S)$. For the function $F$, the proof will be performed for $(t, S)$. Thus

$$
\left|F_{1}(t, S)-F_{1}\left(t, S_{1}\right)\right|^{2}=\left|\delta(t)\left(\alpha I+w\left(\beta I_{D}+\gamma I_{A}+\delta_{1} I_{R}+\delta_{2} I_{T}\right)+\gamma_{1}+\mu_{1}\right)\left(S-S_{1}\right)\right|^{2} .
$$

We define the following norm:

$$
\|\varphi\|_{\infty}=\sup _{t \in[0, T]}|\varphi|^{2}
$$

then

$$
\begin{aligned}
\left|F_{1}(S, t)-F_{1}\left(S_{1}, t\right)\right|^{2} & \leq \sup _{t \in[0, T]}\left|\delta(t)\left(\alpha I+w\left(\beta I_{D}+\gamma I_{A}+\delta_{1} I_{R}+\delta_{2} I_{T}\right)\right)\left(S-S_{1}\right)\right|^{2} \\
& \leq\left\|\delta(t)\left(\alpha I+w\left(\beta I_{D}+\gamma I_{A}+\delta_{1} I_{R}+\delta_{2} I_{T}\right)\right)\right\|_{\infty}^{2}\left|S-S_{1}\right|^{2} \\
& \leq K_{1}\left|S-S_{1}\right|^{2}
\end{aligned}
$$

and

$$
\begin{aligned}
\left|G_{1}(S, t)-G_{1}\left(S_{1}, t\right)\right|^{2} & =\left|\left(\Pi_{11} S+\Pi_{12}\right) S-\left(\Pi_{11} S_{1}+\Pi_{12}\right) S_{1}\right|^{2} \\
& =\left|\Pi_{11}\left(S^{2}-S_{1}^{2}\right)-\Pi_{12}\left(S-S_{1}\right)\right|^{2} \\
& =\left(\Pi_{11}\left(S+S_{1}\right)+\Pi_{12}\right)^{2}\left|S-S_{1}\right|^{2} \\
& =\left(\Pi_{11}^{2}\left(S+S_{1}\right)^{2}+2 \Pi_{11} \Pi_{12}\left(S+S_{1}\right)+\Pi_{12}^{2}\right)\left|S-S_{1}\right|^{2} \\
& =\left(\Pi_{11}^{2}\left(S^{2}+2 S S_{1}+S_{1}^{2}\right)+2 \Pi_{11} \Pi_{12}\left(S+S_{1}\right)+\Pi_{12}^{2}\right)\left|S-S_{1}\right|^{2}
\end{aligned}
$$




$$
\begin{aligned}
& \leq\left\{\begin{array}{c}
\Pi_{11}^{2}\left(\begin{array}{c}
\sup _{t \in[0, T]}\left|S^{2}(t)\right|+2 \sup _{t \in[0, T]}|S(t)| \sup _{t \in[0, T]}\left|S_{1}(t)\right| \\
+\sup _{t \in[0, T]}\left|S_{1}^{2}(t)\right|
\end{array}\right) \\
+2 \Pi_{11} \Pi_{12}\left(\sup _{t \in[0, T]}|S(t)|+\sup _{t \in[0, T]}\left|S_{1}(t)\right|\right)+\Pi_{12}^{2}
\end{array}\right\} \\
& \times\left|S-S_{1}\right|^{2} \\
& \leq\left\{\begin{array}{c}
\Pi_{11}^{2}\left(\left\|S^{2}\right\|_{\infty}+2\|S\|_{\infty}\left\|S_{1}\right\|_{\infty}+\left\|S_{1}^{2}\right\|_{\infty}\right) \\
+2 \Pi_{11} \Pi_{12}\|S\|_{\infty}\left\|S_{1}\right\|_{\infty}+\Pi_{12}^{2}
\end{array}\right\}\left|S-S_{1}\right|^{2} \\
& \leq \bar{K}_{1}\left|S-S_{1}\right|^{2} \text {, }
\end{aligned}
$$

where

$$
\begin{aligned}
\bar{K}_{1} & =\Pi_{11}^{2}\left(\left\|S^{2}\right\|_{\infty}+2\|S\|_{\infty}\left\|S_{1}\right\|_{\infty}+\left\|S_{1}^{2}\right\|_{\infty}\right)+2 \Pi_{11} \Pi_{12}\|S\|_{\infty}\left\|S_{1}\right\|_{\infty}+\Pi_{12}^{2} \\
& =\Pi_{11}^{2}\left(\|S\|_{\infty}+\left\|S_{1}\right\|_{\infty}\right)^{2}+2 \Pi_{11} \Pi_{12}\|S\|_{\infty}\left\|S_{1}\right\|_{\infty}+\Pi_{12}^{2} .
\end{aligned}
$$

Similarly,

$$
\begin{aligned}
& \bar{K}_{2}=\Pi_{21}^{2}\left(\|I\|_{\infty}+\left\|I_{1}\right\|_{\infty}\right)^{2}+2 \Pi_{21} \Pi_{22}\|I\|_{\infty}\left\|I_{1}\right\|_{\infty}+\Pi_{22}^{2}, \\
& \bar{K}_{3}=\Pi_{31}^{2}\left(\left\|I_{A}\right\|_{\infty}+\left\|I_{A 1}\right\|_{\infty}\right)^{2}+2 \Pi_{31} \Pi_{32}\left\|I_{A}\right\|_{\infty}\left\|I_{A 1}\right\|_{\infty}+\Pi_{32}^{2}, \\
& \bar{K}_{4}=\Pi_{41}^{2}\left(\left\|I_{D}\right\|_{\infty}+\left\|I_{D 1}\right\|_{\infty}\right)^{2}+2 \Pi_{41} \Pi_{42}\left\|I_{D}\right\|_{\infty}\left\|I_{D 1}\right\|_{\infty}+\Pi_{42}^{2}, \\
& \bar{K}_{5}=\Pi_{51}^{2}\left(\left\|I_{R}\right\|_{\infty}+\left\|I_{R 1}\right\|_{\infty}\right)^{2}+2 \Pi_{51} \Pi_{52}\left\|I_{R}\right\|_{\infty}\left\|I_{R 1}\right\|_{\infty}+\Pi_{52}^{2}, \\
& \bar{K}_{6}=\Pi_{61}^{2}\left(\left\|I_{T}\right\|_{\infty}+\left\|I_{T 1}\right\|_{\infty}\right)^{2}+2 \Pi_{61} \Pi_{62}\left\|I_{T}\right\|_{\infty}\left\|I_{T 1}\right\|_{\infty}+\Pi_{62}^{2}, \\
& \bar{K}_{7}=\Pi_{71}^{2}\left(\|R\|_{\infty}+\left\|R_{1}\right\|_{\infty}\right)^{2}+2 \Pi_{71} \Pi_{72}\|R\|_{\infty}\left\|R_{1}\right\|_{\infty}+\Pi_{72}^{2}, \\
& \bar{K}_{8}=\Pi_{81}^{2}\left(\|V\|_{\infty}+\left\|V_{1}\right\|_{\infty}\right)^{2}+2 \Pi_{81} \Pi_{82}\|V\|_{\infty}\left\|V_{1}\right\|_{\infty}+\Pi_{82}^{2} .
\end{aligned}
$$

Also

$$
\begin{aligned}
\left|F_{2}(I, t)-F_{2}\left(I_{1}, t\right)\right|^{2} & =\left|\delta(t) \alpha\left(I-I_{1}\right)-\left(\varepsilon+\xi+\lambda+\mu_{1}\right)\left(I-I_{1}\right)\right|^{2} \\
& =\left|\left(\delta(t) \alpha-\left(\varepsilon+\xi+\lambda+\mu_{1}\right)\right)\left(I-I_{1}\right)\right|^{2} \\
& \leq \sup _{t \in[0, T]}\left|\left(\delta(t) \alpha-\left(\varepsilon+\xi+\lambda+\mu_{1}\right)\right)\right|^{2}\left|I-I_{1}\right|^{2} \\
& \leq\|\delta(t)\|_{\infty}\left|\alpha-\left(\varepsilon+\xi+\lambda+\mu_{1}\right)\right|^{2}\left|I-I_{1}\right|^{2} \\
& \leq K_{2}\left|I-I_{1}\right|^{2}
\end{aligned}
$$

where

$$
K_{2}=\|\delta(t)\|_{\infty}\left|\alpha-\left(\varepsilon+\xi+\lambda+\mu_{1}\right)\right|^{2} .
$$

Also

$$
\begin{aligned}
\left|F_{3}\left(I_{A}, t\right)-F_{3}\left(I_{A 1}, t\right)\right|^{2} & =\left|-\left(\theta+\mu+\chi+\mu_{1}\right)\left(I_{A}-I_{A 1}\right)\right|^{2} \\
& \leq 2\left|\left(\theta+\mu+\chi+\mu_{1}\right)\right|^{2}\left|I_{A}-I_{A 1}\right|^{2} \\
& \leq K_{3}\left|I_{A}-I_{A 1}\right|^{2},
\end{aligned}
$$


where

$$
K_{3}=2\left|\left(\theta+\mu+\chi+\mu_{1}\right)\right|^{2} .
$$

Similarly, we evaluate

$$
\begin{aligned}
\left|F_{4}\left(I_{D}, t\right)-F_{4}\left(I_{D 1}, t\right)\right|^{2} & =\left|\eta+\varphi+\mu_{1}\right|^{2}\left|I_{D}-I_{D 1}\right|^{2} \\
& \leq K_{4}\left|I_{D}-I_{D 1}\right|^{2}, \\
\left|F_{5}\left(I_{R}, t\right)-F_{5}\left(I_{R 1}, t\right)\right|^{2} & =\left|v+\xi+\mu_{1}\right|^{2}\left|I_{R}-I_{R 1}\right|^{2} \\
& \leq K_{5}\left|I_{R}-I_{R 1}\right|^{2}, \\
\left|F_{6}\left(I_{T}, t\right)-F_{6}\left(I_{T 1}, t\right)\right|^{2} & =\left|\sigma+\tau+\mu_{1}\right|^{2}\left|I_{T}-I_{T 1}\right|^{2} \\
& \leq K_{6}\left|I_{T}-I_{T 1}\right|, \\
\left|F_{7}(R, t)-F_{7}\left(R_{1}, t\right)\right|^{2} & =\left|\Phi+\mu_{1}\right|^{2}\left|R-R_{1}\right|^{2} \\
& \leq K_{7}\left|R-R_{1}\right|, \\
\left|F_{8}(V, t)-F_{8}\left(V_{1}, t\right)\right|^{2} & =\left|\mu_{1}\right|^{2}\left|V-V_{1}\right|^{2} \\
& \leq K_{8}\left|V-V_{1}\right|^{2} .
\end{aligned}
$$

For both classes $G_{i}$ and $F_{i}$, we have verified condition (i). Now we verify the second condition.

$$
\begin{aligned}
\left|F_{1}(S, t)\right|^{2} & =\left|\Lambda-\delta(t)\left(\alpha I+w\left(\beta I_{D}+\gamma I_{A}+\delta_{1} I_{R}+\delta_{2} I_{T}\right)+\gamma_{1}+\mu_{1}\right) S\right|^{2} \\
& \leq\left|\Lambda S-\delta(t)\left(\alpha I+w\left(\beta I_{D}+\gamma I_{A}+\delta_{1} I_{R}+\delta_{2} I_{T}\right)+\gamma_{1}+\mu_{1}\right) S\right|^{2} \\
& \leq|S|^{2}\left|\Lambda-\delta(t)\left(\alpha I+w\left(\beta I_{D}+\gamma I_{A}+\delta_{1} I_{R}+\delta_{2} I_{T}\right)+\gamma_{1}+\mu_{1}\right)\right|^{2} \\
& <\left(|S|^{2}+1\right)\left|\Lambda-\delta(t)\left(\alpha I+w\left(\beta I_{D}+\gamma I_{A}+\delta_{1} I_{R}+\delta_{2} I_{T}\right)+\gamma_{1}+\mu_{1}\right)\right|^{2} \\
& <\left(|S|^{2}+1\right)\left|\Lambda-\delta(t)\left(\alpha I+w\left(\beta I_{D}+\gamma I_{A}+\delta_{1} I_{R}+\delta_{2} I_{T}\right)+\gamma_{1}+\mu_{1}\right)\right|^{2} \\
& <\left(|S|^{2}+1\right) \sup _{t \in[0, T]}\left|\Lambda-\delta(t)\left(\alpha I+w\left(\beta I_{D}+\gamma I_{A}+\delta_{1} I_{R}+\delta_{2} I_{T}\right)+\gamma_{1}+\mu_{1}\right)\right|^{2} \\
& <K^{1}\left(|S|^{2}+1\right),
\end{aligned}
$$

where

$$
K^{1}=\sup _{t \in[0, T]}\left|\Lambda-\delta(t)\left(\alpha I+w\left(\beta I_{D}+\gamma I_{A}+\delta_{1} I_{R}+\delta_{2} I_{T}\right)+\gamma_{1}+\mu_{1}\right)\right|^{2} .
$$

Then

$$
\begin{aligned}
\left|G_{1}(S, t)-G_{1}\left(S_{1}, t\right)\right|^{2} & =\left|\left(\Pi_{11} S+\Pi_{12}\right) S\right|^{2} \\
& \leq\left|\Pi_{11} S^{2}+\Pi_{12} S^{2}\right|^{2} \\
& \leq\left(\Pi_{11}+\Pi_{12}\right)^{2}\left|S^{2}\right|^{2} \\
& \leq\left(\Pi_{11}+\Pi_{12}\right)^{2} \sup _{t \in[0, T]}\left|S^{2}\right||S|^{2}
\end{aligned}
$$




$$
\begin{aligned}
& \leq\left(\Pi_{11}+\Pi_{12}\right)^{2}\left\|S^{2}\right\|_{\infty}\left(|S|^{2}+1\right) \\
& \leq \bar{K}^{1}\left(|S|^{2}+1\right),
\end{aligned}
$$

where

$$
\bar{K}^{1}=\left(\Pi_{11}+\Pi_{12}\right)^{2}\left\|S^{2}\right\|_{\infty}
$$

Similarly,

$$
\begin{aligned}
& \bar{K}^{2}=\left(\Pi_{21}+\Pi_{22}\right)^{2}\left\|I^{2}\right\|_{\infty}, \\
& \bar{K}^{3}=\left(\Pi_{31}+\Pi_{32}\right)^{2}\left\|I_{A}^{2}\right\|_{\infty}, \\
& \bar{K}^{4}=\left(\Pi_{41}+\Pi_{42}\right)^{2}\left\|I_{D}^{2}\right\|_{\infty}, \\
& \bar{K}^{5}=\left(\Pi_{51}+\Pi_{52}\right)^{2}\left\|I_{R}^{2}\right\|_{\infty}, \\
& \bar{K}^{6}=\left(\Pi_{61}+\Pi_{62}\right)^{2}\left\|I_{T}^{2}\right\|_{\infty}, \\
& \bar{K}^{7}=\left(\Pi_{71}+\Pi_{72}\right)^{2}\left\|R^{2}\right\|_{\infty}, \\
& \bar{K}^{8}=\left(\Pi_{81}+\Pi_{82}\right)^{2}\left\|V^{2}\right\|_{\infty} .
\end{aligned}
$$

Also, we have

$$
\begin{aligned}
\left|F_{2}(I, t)\right|^{2}= & \left|\delta(t)\left(w\left(\beta I_{D}+\gamma I_{A}+\delta_{1} I_{R}+\delta_{2} I_{T}\right)\right) S+\delta(t) \alpha I S-\left(\varepsilon+\xi+\lambda+\mu_{1}\right) I\right|^{2} \\
\leq & \left|\delta(t)\left(w\left(\beta I_{D}+\gamma I_{A}+\delta_{1} I_{R}+\delta_{2} I_{T}\right)\right) S+\delta(t) \alpha S-\left(\varepsilon+\xi+\lambda+\mu_{1}\right)\right||I|^{2} \\
< & \left(|S|^{2}+1\right) \sup _{t \in[0, T]} \mid \delta(t)\left(w\left(\beta I_{D}+\gamma I_{A}+\delta_{1} I_{R}+\delta_{2} I_{T}\right)\right) S+\delta(t) \alpha S \\
& -\left.\left(\varepsilon+\xi+\lambda+\mu_{1}\right)\right|^{2} \\
< & K^{2}\left(|I|^{2}+1\right), \\
\left|F_{3}\left(I_{A}, t\right)\right|^{2}= & \left|\xi I-\left(\theta+\mu+\chi+\mu_{1}\right) I_{A}\right|^{2} \\
\leq & \left|\xi I-\left(\theta+\mu+\chi+\mu_{1}\right)\right|^{2}\left|I_{A}\right|^{2} \\
\leq & \left(\left|I_{A}\right|^{2}+1\right) \sup _{t \in[0, T]}\left|\xi I-\left(\theta+\mu+\chi+\mu_{1}\right)\right|^{2} \\
\leq & K^{3}\left(\left|I_{A}\right|^{2}+1\right), \\
\left|F_{4}\left(I_{A}, t\right)\right|^{2}= & \left|\varepsilon I-\left(\eta+\varphi+\mu_{1}\right) I_{D}\right|^{2} \\
\leq & \left(\left|I_{D}\right|^{2}+1\right) \sup _{t \in[0, T]}\left|\varepsilon I-\left(\eta+\varphi+\mu_{1}\right)\right|^{2} \\
\leq & K^{4}\left(\left|I_{D}\right|^{2}+1\right), \\
\left|F_{5}\left(I_{R}, t\right)\right|^{2} \leq & \left(\left|I_{R}\right|^{2}+1\right) \sup _{t \in[0, T]}\left|\eta I_{D}+\theta I_{A}-\left(v+\xi+\mu_{1}\right)\right|^{2} \\
\leq & K^{5}\left(\left|I_{R}\right|^{2}+1\right),
\end{aligned}
$$




$$
\begin{aligned}
\left|F_{6}\left(I_{T}, t\right)\right|^{2} & \leq\left(\left|I_{T}\right|^{2}+1\right) \sup _{t \in[0, T]}\left|\mu I_{A}+v I_{R}-\left(\sigma+\tau+\mu_{1}\right)\right|^{2} \\
& \leq K^{6}\left(\left|I_{T}\right|^{2}+1\right), \\
\left|F_{6}\left(I_{T}, t\right)\right|^{2} & \leq\left(\left|I_{T}\right|^{2}+1\right) \sup _{t \in[0, T]}\left|\mu I_{A}+v I_{R}-\left(\sigma+\tau+\mu_{1}\right)\right|^{2} \\
& \leq K^{6}\left(\left|I_{T}\right|^{2}+1\right), \\
\left|F_{7}(R, t)\right|^{2} & \leq\left(|R|^{2}+1\right) \sup _{t \in[0, T]}\left|\lambda I+\varphi I_{D}+\chi I_{A}+\xi I_{R}+\sigma I_{T}-\left(\Phi+\mu_{1}\right)\right|^{2} \\
& \leq K^{7}\left(|R|^{2}+1\right) .
\end{aligned}
$$

Finally, we have

$$
\begin{aligned}
\left|F_{8}(V, t)\right|^{2} & \leq\left(|V|^{2}+1\right) \sup _{t \in[0, T]}\left|\gamma_{1} S+\Phi R-\mu_{1}\right|^{2} \\
& \leq K^{8}\left(|V|^{2}+1\right) .
\end{aligned}
$$

Both $G_{i}$ and $F_{i}$ verify the second condition. Therefore, according to the above theorem, the system has a unique system solution.

\subsection{Numerical simulation for the stochastic model}

Numerical solutions of the suggested stochastic model are presented in Figs. 22-25. The numerical solution depicts the future stochastic behavior of the susceptible class, five subclasses of the infected population, the recovered class, the death class, and the vaccination class. These are depicted in figures below.

\section{Atangana-Seda modified scheme}

The mathematical model considered in this work has the ability to depict two to three waves of COVID-19 spread. The model is subjected to a system of initial conditions. Additionally, the model is nonlinear, thus it is impossible to obtain exact solutions to the system, thus numerical schemes are needed. We present a numerical scheme based on the Newton polynomial [18]. However, one needs the initial condition and two additional components for the scheme to be implemented. In this section, we present a modified version that will not need the two additional components, and then the scheme will be used later to provide numerical solutions for the suggested COVID-19 model with different differential operators. We start with the classical case, the following is considered:

$$
\frac{d y(t)}{d t}=f(t, y(t))
$$

Then

$$
y^{n+1}=y^{n}+\left\{\frac{5}{12} f\left(t_{n-2}, y^{n-2}\right)-\frac{4}{3} f\left(t_{n-1}, y^{n-1}\right)+\frac{5}{12} f\left(t_{n}, y^{n}\right)\right\} \Delta t .
$$

To reduce these requirements, we proceed as follows:

$$
\frac{y^{n}-y^{n-1}}{\Delta t}=f\left(t_{n}, y^{n}\right) \quad \Rightarrow \quad y^{n-1}=y^{n}-f\left(t_{n}, y^{n}\right) \Delta t .
$$



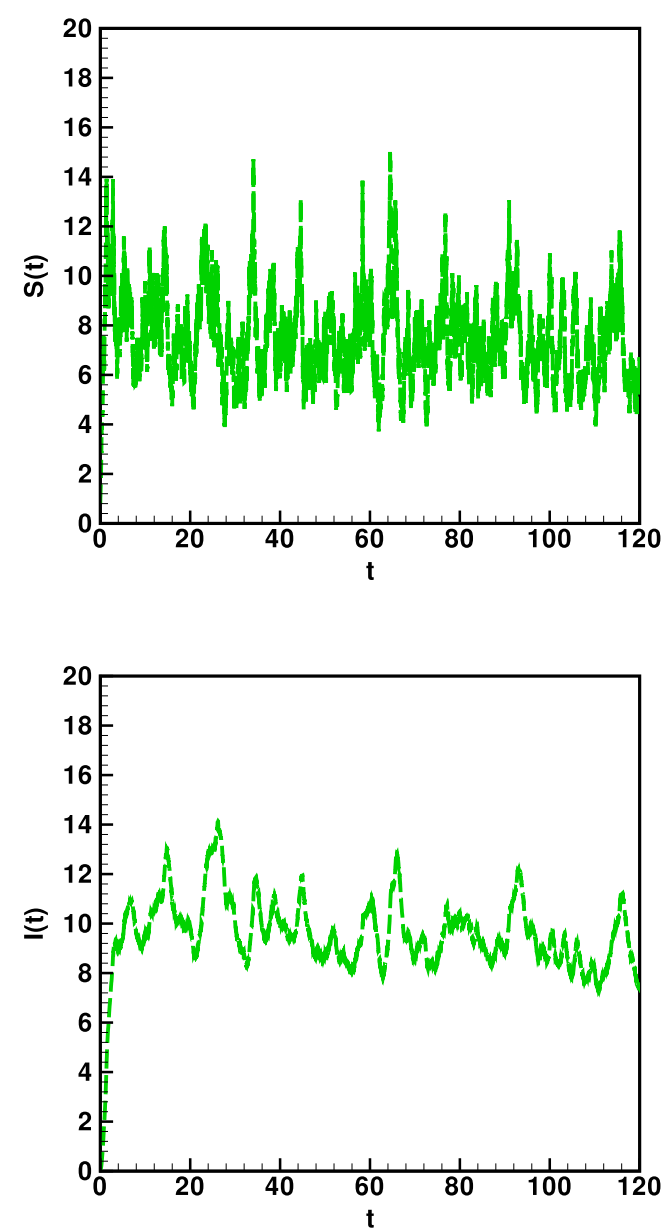

Figure 22 Stochastic behavior of $S(t)$ and $/(t)$ classes

On the other hand,

$$
\frac{y^{n-1}-y^{n-2}}{\Delta t}=f\left(t_{n-1}, y^{n-1}\right)
$$

or

$$
\begin{aligned}
y^{n-2} & =y^{n-1}-f\left(t_{n-1}, y^{n-1}\right) \Delta t \\
& =y^{n}-\Delta t f\left(t_{n}, y^{n}\right)-\Delta t f\left(t_{n-1}, y^{n}-f\left(t_{n}, y^{n}\right) \Delta t\right) .
\end{aligned}
$$

Replacing $y^{n-2}$ and $y^{n-1}$ with their values, we obtain

$$
\begin{aligned}
y^{n+1}= & y^{n}+\frac{5}{12} \Delta t f\left(t_{n-2}, y^{n}-\Delta t f\left(t_{n}, y^{n}\right)-\Delta t f\left(t_{n-1}, y^{n}-f\left(t_{n}, y^{n}\right) \Delta t\right)\right) \\
& -\frac{4}{3} f\left(t_{n-1}, y^{n}-f\left(t_{n}, y^{n}\right) \Delta t\right)+\frac{23}{12} f\left(t_{n}, y^{n}\right) \Delta t .
\end{aligned}
$$

The above does not need $y^{1}$ and $y^{2}$, only the initial condition. With the Caputo-Fabrizio derivative, we consider the following:

$$
{ }_{0}^{C F} D_{t}^{\alpha} y(t)=f(t, y(t)) \text {. }
$$



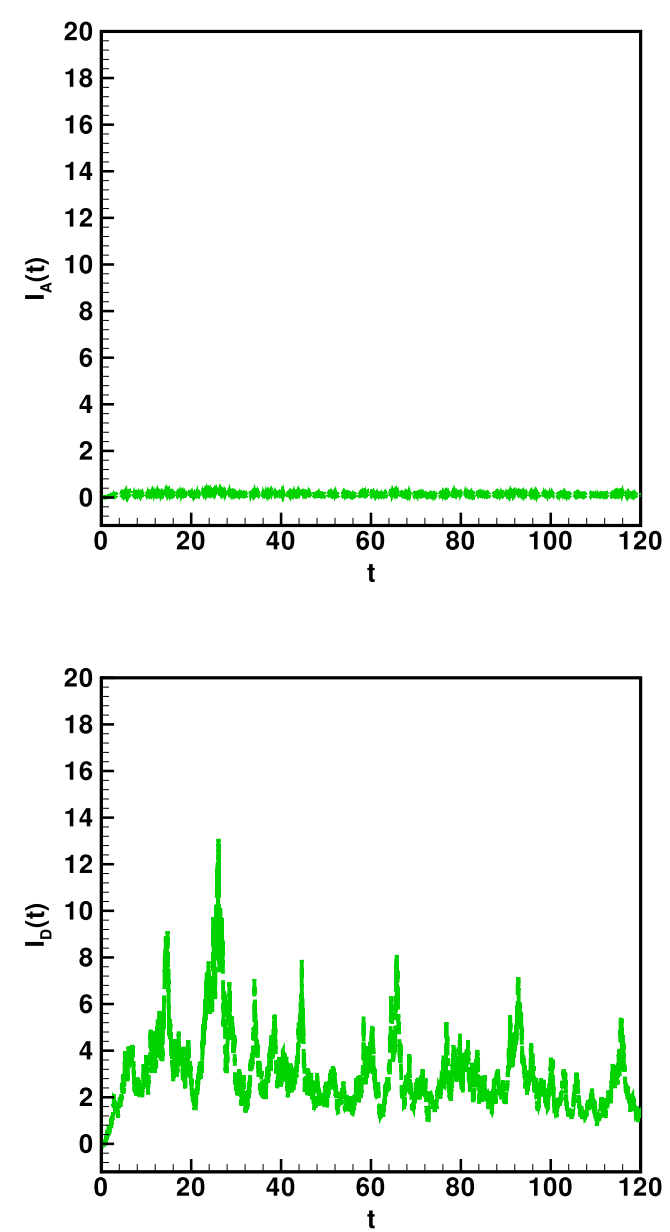

Figure 23 Stochastic behavior of $I_{A}(t)$ and $I_{D}(t)$ classes

From the definition of the Caputo-Fabrizio integral, we can reformulate the above equation as follows:

$$
y(t)-y(0)=\frac{1-\alpha}{M(\alpha)} f(t, y(t))+\frac{\alpha}{M(\alpha)} \int_{0}^{t} f(\tau, y(\tau)) d \tau .
$$

We have, at the point $t_{n+1}=(n+1) \Delta t$,

$$
y\left(t_{n+1}\right)-y(0)=\frac{1-\alpha}{M(\alpha)} f\left(t_{n+1}, y\left(t_{n+1}\right)\right)+\frac{\alpha}{M(\alpha)} \int_{0}^{t_{n+1}} f(\tau, y(\tau)) d \tau,
$$

and at the point $t_{n}=n \Delta t$,

$$
y\left(t_{n}\right)-y(0)=\frac{1-\alpha}{M(\alpha)} f\left(t_{n}, y\left(t_{n}\right)\right)+\frac{\alpha}{M(\alpha)} \int_{0}^{t_{n}} f(\tau, y(\tau)) d \tau .
$$

Taking the difference of these equations, we can write the following:

$$
\begin{aligned}
y\left(t_{n+1}\right)-y\left(t_{n}\right)= & \frac{1-\alpha}{M(\alpha)}\left[f\left(t_{n+1}, y\left(t_{n+1}\right)\right)-f\left(t_{n}, y\left(t_{n}\right)\right)\right] \\
& +\frac{\alpha}{M(\alpha)} \int_{t_{n}}^{t_{n+1}} f(\tau, y(\tau)) d \tau
\end{aligned}
$$



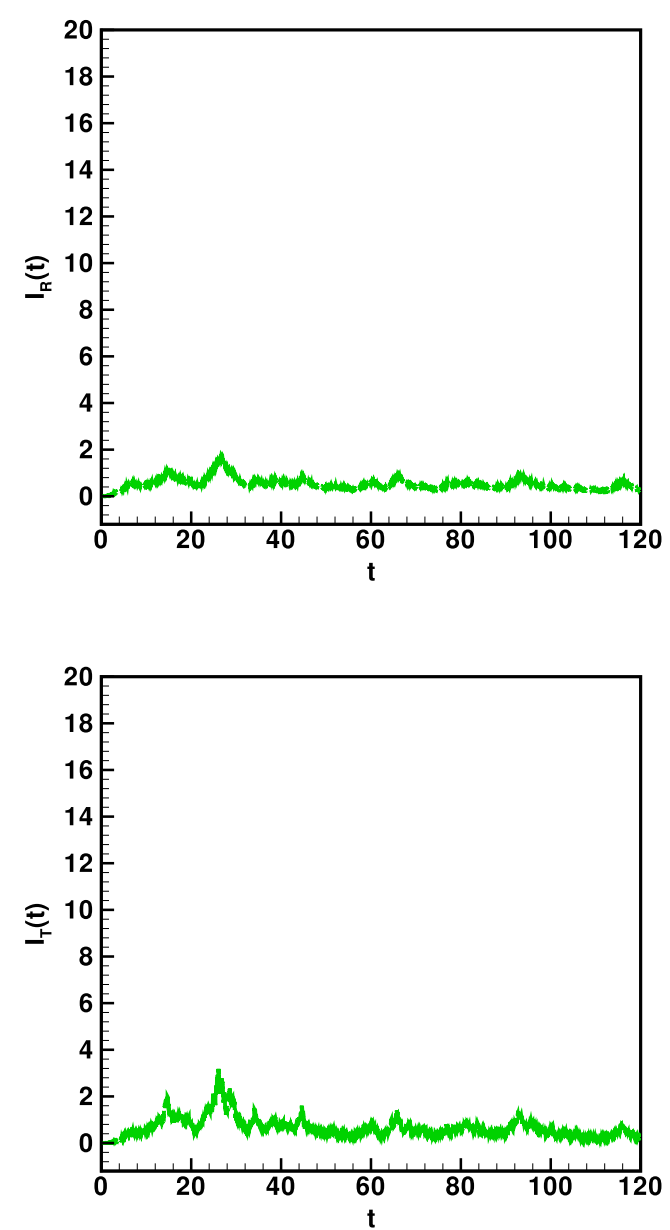

Figure 24 Stochastic behavior of $I_{R}(t)$ and $I_{T}(t)$ classes

$$
\begin{aligned}
& =\frac{1-\alpha}{M(\alpha)}\left[\begin{array}{c}
f\left(t_{n+1}, y\left(t_{n}\right)-\Delta t f\left(t_{n}, y\left(t_{n}\right)\right)\right) \\
-f\left(t_{n}, y\left(t_{n}\right)\right)
\end{array}\right] \\
& +\frac{\alpha}{M(\alpha)}\left\{\begin{array}{c}
\frac{5}{12} f\left(t_{n-2}, y^{n}-\Delta t f\left(t_{n}, y^{n}\right)-\Delta t f\left(t_{n-1}, y^{n}-f\left(t_{n}, y^{n}\right) \Delta t\right)\right) \Delta t \\
-\frac{4}{3} f\left(t_{n-1}, y^{n}-f\left(t_{n}, y^{n}\right) \Delta t\right) \Delta t \\
+\frac{23}{12} f\left(t_{n}, y^{n}\right) \Delta t
\end{array}\right\} .
\end{aligned}
$$

With the Caputo derivative, we write

$$
\left\{\begin{array}{l}
{ }_{0}^{C} D_{t}^{\alpha} y(t)=f(t, y(t)), \\
y(0)=y_{0} .
\end{array}\right.
$$

We convert the above into

$$
y(t)-y(0)=\frac{1}{\Gamma(\alpha)} \int_{0}^{t} f(\tau, y(\tau))(t-\tau)^{\alpha-1} d \tau .
$$

At the point $t_{n+1}=(n+1) \Delta t$, we have the following:

$$
y\left(t_{n+1}\right)-y(0)=\frac{1}{\Gamma(\alpha)} \int_{0}^{t_{n+1}} f(\tau, y(\tau))\left(t_{n+1}-\tau\right)^{\alpha-1} d \tau,
$$



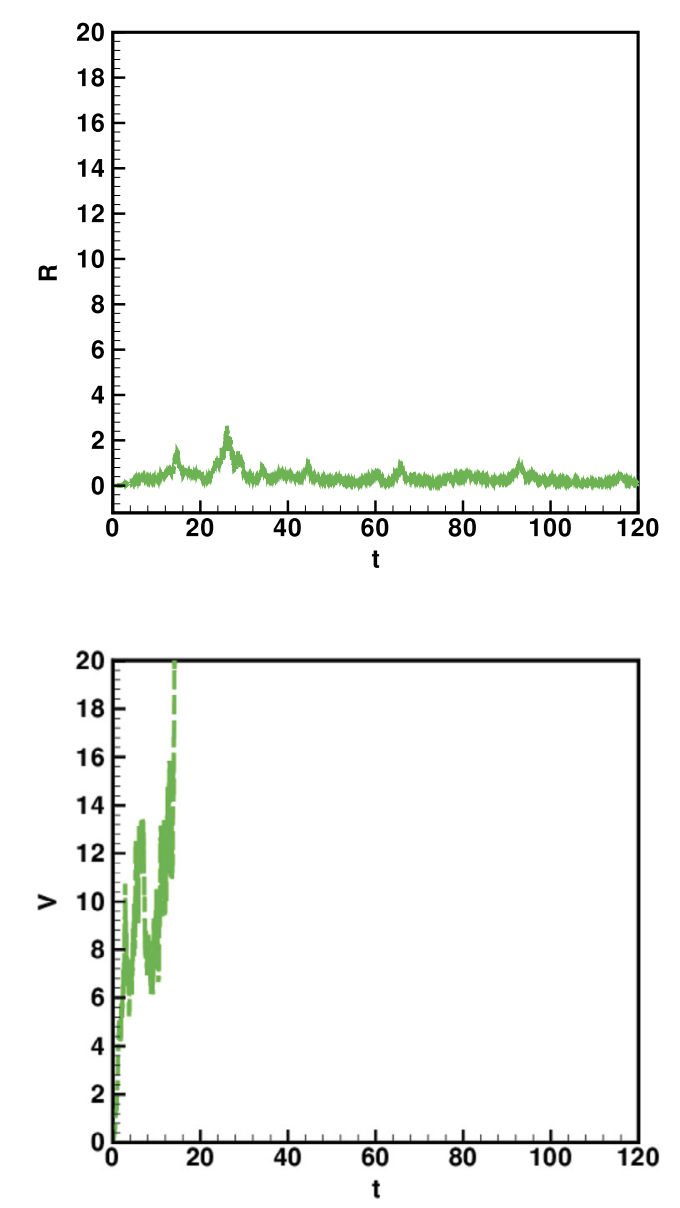

Figure 25 Stochastic behavior of $R(t)$ and $V(t)$ classes

and we write

$$
y\left(t_{n+1}\right)=y(0)+\frac{1}{\Gamma(\alpha)} \sum_{j=2}^{n} \int_{t_{j}}^{t_{j+1}} f(\tau, y(\tau))\left(t_{n+1}-\tau\right)^{\alpha-1} d \tau .
$$

After putting the Newton polynomial into the above equation, the above equation can be written as follows:

$$
\begin{aligned}
y^{n+1}= & y^{0}+\frac{(\Delta t)^{\alpha}}{\Gamma(\alpha+1)} \sum_{j=2}^{n} f\left(t_{j-2}, y^{j-2}\right)\left[(n-j+1)^{\alpha}-(n-j)^{\alpha}\right] \\
& +\frac{(\Delta t)^{\alpha}}{\Gamma(\alpha+2)} \sum_{j=2}^{n}\left[f\left(t_{j-1}, y^{j-1}\right)-f\left(t_{j-2}, y^{j-2}\right)\right] \\
& \times\left[\begin{array}{c}
(n-j+1)^{\alpha}(n-j+3+2 \alpha) \\
-(n-j)^{\alpha}(n-j+3+3 \alpha)
\end{array}\right] \\
& +\frac{\alpha(\Delta t)^{\alpha}}{2 \Gamma(\alpha+3)} \sum_{j=2}^{n}\left[\begin{array}{c}
f\left(t_{j}, y^{j}\right)-2 f\left(t_{j-1}, y^{j-1}\right) \\
+f\left(t_{j-2}, y^{j-2}\right)
\end{array}\right] \\
& \times\left[\begin{array}{c}
\left.(n-j+1)^{\alpha}\left[\begin{array}{c}
2(n-j)^{2}+(3 \alpha+10)(n-j) \\
+2 \alpha^{2}+9 \alpha+12
\end{array}\right]\right], \\
-(n-j)^{\alpha}\left[\begin{array}{c}
2(n-j)^{2}+(5 \alpha+10)(n-j) \\
+6 \alpha^{2}+18 \alpha+12
\end{array}\right]
\end{array}\right]
\end{aligned}
$$


where

$$
\begin{aligned}
& f\left(t_{j-1}, y^{j-1}\right)=f\left(t_{j-1}, y^{j}-f\left(t_{j}, y^{j}\right) \Delta t\right) \\
& f\left(t_{j-2}, y^{j-2}\right)=f\left(t_{j-2}, y^{j}-\Delta t f\left(t_{j}, y^{j}\right)-\Delta t f\left(t_{j-1}, y^{j}-f\left(t_{j}, y^{j}\right) \Delta t\right)\right) .
\end{aligned}
$$

With Atangana-Baleanu, we have

$$
\left\{\begin{array}{l}
{ }_{0}^{A B C} D_{t}^{\alpha} y(t)=f(t, y(t)), \\
y(0)=y_{0}
\end{array}\right.
$$

We transform the above equation into

$$
y(t)-y(0)=\frac{1-\alpha}{A B(\alpha)} f(t, y(t))+\frac{\alpha}{A B(\alpha) \Gamma(\alpha)} \int_{0}^{t} f(\tau, y(\tau))(t-\tau)^{\alpha-1} d \tau .
$$

At the point $t_{n+1}=(n+1) \Delta t$, we have the following:

$$
\begin{aligned}
y\left(t_{n+1}\right)-y(0)= & \frac{1-\alpha}{A B(\alpha)} f(t, y(t)) \\
& +\frac{\alpha}{A B(\alpha) \Gamma(\alpha)} \int_{0}^{t_{n+1}} f(\tau, y(\tau))\left(t_{n+1}-\tau\right)^{\alpha-1} d \tau,
\end{aligned}
$$

and we write

$$
\begin{aligned}
y\left(t_{n+1}\right)= & y(0)+\frac{1-\alpha}{A B(\alpha)} f\left(t_{n+1}, y^{n+1}\right) \\
& +\frac{\alpha}{A B(\alpha) \Gamma(\alpha)} \sum_{j=2}^{n} \int_{t_{j}}^{t_{j+1}} f(\tau, y(\tau))\left(t_{n+1}-\tau\right)^{\alpha-1} d \tau .
\end{aligned}
$$

After putting the Newton polynomial into the above equation, the above equation can be written as follows:

$$
\begin{aligned}
& y^{n+1}=y^{0}+\frac{1-\alpha}{A B(\alpha)} f\left(t_{n+1}, y\left(t_{n}\right)-\Delta t f\left(t_{n}, y\left(t_{n}\right)\right)\right) \\
& +\frac{\alpha(\Delta t)^{\alpha}}{A B(\alpha) \Gamma(\alpha+1)} \sum_{j=2}^{n} f\left(\begin{array}{c}
t_{j-2}, y^{j}-\Delta t f\left(t_{j}, y^{j}\right) \\
-\Delta t f\left(t_{j-1}, y^{j}-f\left(t_{j}, y^{j}\right) \Delta t\right)
\end{array}\right) \\
& \times\left[(n-j+1)^{\alpha}-(n-j)^{\alpha}\right] \\
& +\frac{\alpha(\Delta t)^{\alpha}}{A B(\alpha) \Gamma(\alpha+2)} \sum_{j=2}^{n}\left[\begin{array}{c}
f\left(t_{j-1}, y^{j}-f\left(t_{j}, y^{j}\right) \Delta t\right) \\
-f\left(t_{j-2}, y^{j}-\Delta t f\left(t_{j}, y^{j}\right)-\Delta t f\left(t_{j-1}, y^{j}-f\left(t_{j}, y^{j}\right) \Delta t\right)\right)
\end{array}\right] \\
& \times\left[\begin{array}{c}
(n-j+1)^{\alpha}(n-j+3+2 \alpha) \\
-(n-j)^{\alpha}(n-j+3+3 \alpha)
\end{array}\right] \\
& +\frac{\alpha(\Delta t)^{\alpha}}{2 A B(\alpha) \Gamma(\alpha+3)} \sum_{j=2}^{n}\left[\begin{array}{c}
f\left(t_{j}, y^{j}\right)-2 f\left(t_{j-1}, y^{j-1}\right) \\
+f\left(\begin{array}{c}
t_{j-2}, y^{j}-\Delta t f\left(t_{j}, y^{j}\right) \\
-\Delta t f\left(t_{j-1}, y^{j}-f\left(t_{j}, y^{j}\right) \Delta t\right)
\end{array}\right)
\end{array}\right]
\end{aligned}
$$




$$
\times\left[\begin{array}{c}
(n-j+1)^{\alpha}\left[\begin{array}{c}
2(n-j)^{2}+(3 \alpha+10)(n-j) \\
+2 \alpha^{2}+9 \alpha+12
\end{array}\right] \\
-(n-j)^{\alpha}\left[\begin{array}{c}
2(n-j)^{2}+(5 \alpha+10)(n-j) \\
+6 \alpha^{2}+18 \alpha+12
\end{array}\right]
\end{array}\right] .
$$

With the Caputo-Fabrizio fractal-fractional derivative, we consider

$$
\begin{aligned}
& { }_{0}^{F F E} D_{t}^{\alpha, \beta} y(t)=f(t, y(t)), \\
& y(0)=y_{0} .
\end{aligned}
$$

Applying the associated integral operator with exponential kernel, we can reformulate equation (69) as follows:

$$
y(t)=\frac{1-\alpha}{M(\alpha)} t^{1-\beta} f(t, y(t))+\frac{\alpha}{M(\alpha)} \int_{0}^{t} f(\tau, y(\tau)) \tau^{1-\beta} d \tau .
$$

At the point $t_{n+1}=(n+1) \Delta t$,

$$
y\left(t_{n+1}\right)=\frac{1-\alpha}{M(\alpha)} t_{n+1}^{1-\beta} f\left(t_{n+1}, y\left(t_{n+1}\right)\right)+\frac{\alpha}{M(\alpha)} \int_{0}^{t_{n+1}} f(\tau, y(\tau)) \tau^{1-\beta} d \tau,
$$

and at the point $t_{n}=n \Delta t$, we have

$$
y\left(t_{n}\right)=\frac{1-\alpha}{M(\alpha)} t_{n}^{1-\beta} f\left(t_{n}, y\left(t_{n}\right)\right)+\frac{\alpha}{M(\alpha)} \int_{0}^{t_{n}} f(\tau, y(\tau)) \tau^{1-\beta} d \tau
$$

If we take the difference of these equations, we obtain the following equation:

$$
\begin{aligned}
y\left(t_{n+1}\right)-y\left(t_{n}\right)= & \frac{1-\alpha}{M(\alpha)}\left[\begin{array}{c}
t_{n+1}^{1-\beta} f\left(t_{n+1}, y\left(t_{n+1}\right)\right) \\
-t_{n}^{1-\beta} f\left(t_{n}, y\left(t_{n}\right)\right)
\end{array}\right] \\
& +\frac{\alpha}{M(\alpha)} \int_{t_{n}}^{t_{n+1}} f(\tau, y(\tau)) \tau^{1-\beta} d \tau .
\end{aligned}
$$

For brevity, we consider

$$
\begin{aligned}
y\left(t_{n+1}\right)-y\left(t_{n}\right)= & \frac{1-\alpha}{M(\alpha)}\left[F\left(t_{n+1}, y\left(t_{n+1}\right)\right)-F\left(t_{n}, y\left(t_{n}\right)\right)\right] \\
& +\frac{\alpha}{M(\alpha)} \int_{t_{n}}^{t_{n+1}} F(\tau, y(\tau)) d \tau,
\end{aligned}
$$

where

$$
F(t, y(t))=f(t, y(t)) t^{1-\beta}
$$

We can rearrange the above scheme as follows:

$$
y^{n+1}-y^{n}=\frac{1-\alpha}{M(\alpha)}\left[\begin{array}{c}
F\left(t_{n+1}, y\left(t_{n}\right)-\Delta t f\left(t_{n}, y\left(t_{n}\right)\right)\right) \\
-F\left(t_{n}, y\left(t_{n}\right)\right)
\end{array}\right]
$$




$$
+\frac{\alpha}{M(\alpha)}\left\{\begin{array}{c}
\frac{5}{12} F\left(t_{n-2}, y^{n}-\Delta t f\left(t_{n}, y^{n}\right)-\Delta t F\left(t_{n-1}, y^{n}-f\left(t_{n}, y^{n}\right) \Delta t\right)\right) \Delta t \\
-\frac{4}{3} F\left(t_{n-1}, y^{n}-f\left(t_{n}, y^{n}\right) \Delta t\right) \Delta t \\
+\frac{23}{12} F\left(t_{n}, y^{n}\right) \Delta t
\end{array}\right\}
$$

If we replace $F(t, y(t))$ with its value, we can solve our equation numerically with the following scheme:

$$
\begin{aligned}
y^{n+1}-y^{n}= & \frac{1-\alpha}{M(\alpha)}\left[\begin{array}{c}
t_{n+1}^{1-\beta} f\left(t_{n+1}, y\left(t_{n}\right)-\Delta t f\left(t_{n}, y\left(t_{n}\right)\right)\right) \\
-t_{n}^{1-\beta} f\left(t_{n}, y\left(t_{n}\right)\right)
\end{array}\right] \\
& +\frac{\alpha}{M(\alpha)}\left\{\begin{array}{c}
t_{n-2}^{1-\beta} \frac{5}{12} F\left(t_{n-2}, y^{n}-\Delta t f\left(t_{n}, y^{n}\right)-\Delta t f\left(t_{n-1}, y^{n}-f\left(t_{n}, y^{n}\right) \Delta t\right)\right) \Delta t \\
-\frac{4}{3} t_{n-1}^{1-\beta} f\left(t_{n-1}, y^{n}-f\left(t_{n}, y^{n}\right) \Delta t\right) \Delta t \\
+\frac{23}{12} t_{n}^{1-\beta} f\left(t_{n}, y^{n}\right) \Delta t
\end{array}\right\} .
\end{aligned}
$$

With the Atangana-Baleanu fractal-fractional derivative, we write

$$
\begin{aligned}
& { }_{0}^{F F M} D_{t}^{\alpha, \beta} y(t)=f(t, y(t)), \\
& y(0)=y_{0} .
\end{aligned}
$$

Applying the new fractional integral with Mittag-Leffler kernel, we transform the above equation into

$$
\begin{aligned}
y(t)= & y(0)+\frac{1-\alpha}{A B(\alpha)} t^{1-\beta} f(t, y(t)) \\
& +\frac{\alpha}{A B(\alpha) \Gamma(\alpha)} \int_{0}^{t} f(\tau, y(\tau))(t-\tau)^{\alpha-1} \tau^{1-\beta} d \tau .
\end{aligned}
$$

At the point $t_{n+1}=(n+1) \Delta t$, we obtain the following:

$$
\begin{aligned}
y\left(t_{n+1}\right)= & y(0)+\frac{1-\alpha}{A B(\alpha)} t_{n+1}^{1-\beta} f\left(t_{n+1}, y\left(t_{n+1}\right)\right) \\
& +\frac{\alpha}{A B(\alpha) \Gamma(\alpha)} \int_{0}^{t_{n+1}} f(\tau, y(\tau))\left(t_{n+1}-\tau\right)^{\alpha-1} \tau^{1-\beta} d \tau .
\end{aligned}
$$

For simplicity, we shall take

$$
F(t, y(t))=f(t, y(t)) t^{1-\beta} .
$$

We also have

$$
\begin{aligned}
y\left(t_{n+1}\right)= & y(0)+\frac{1-\alpha}{A B(\alpha)} F\left(t_{n+1}, y\left(t_{n+1}\right)\right) \\
& +\frac{\alpha}{A B(\alpha) \Gamma(\alpha)} \sum_{j=2}^{n} \int_{t_{j}}^{t_{j+1}} F(\tau, y(\tau))\left(t_{n+1}-\tau\right)^{\alpha-1} d \tau .
\end{aligned}
$$

Replacing them into the above equation and substituting $F(t, y(t))=f(t, y(t)) t^{1-\beta}$, we can get the following numerical scheme:

$$
y^{n+1}=\frac{1-\alpha}{A B(\alpha)} t_{n+1}^{1-\beta} f\left(t_{n+1}, y\left(t_{n+1}\right)\right)
$$




$$
\begin{aligned}
& +\frac{\alpha(\Delta t)^{\alpha}}{A B(\alpha) \Gamma(\alpha+1)} \sum_{j=2}^{n} t_{j-2}^{1-\beta} f\left(\begin{array}{c}
t_{j-2}, y^{j}-\Delta t f\left(t_{j}, y^{j}\right) \\
-\Delta t f\left(t_{j-1}, y^{j}-f\left(t_{j}, y^{j}\right) \Delta t\right)
\end{array}\right) \\
& \times\left[(n-j+1)^{\alpha}-(n-j)^{\alpha}\right] \\
& +\frac{\alpha(\Delta t)^{\alpha}}{A B(\alpha) \Gamma(\alpha+2)} \sum_{j=2}^{n}\left[\begin{array}{c}
t_{j-1}^{1-\beta} f\left(t_{j-1}, y^{j}-f\left(t_{j}, y^{j}\right) \Delta t\right) \\
-t_{j-2}^{1-\beta} f\left(\begin{array}{c}
t_{j-2}, y^{j}-\Delta t f\left(t_{j}, y^{j}\right) \\
-\Delta t f\left(t_{j-1}, y^{j}-f\left(t_{j}, y^{j}\right) \Delta t\right)
\end{array}\right)
\end{array}\right] \\
& \times\left[\begin{array}{c}
(n-j+1)^{\alpha}(n-j+3+2 \alpha) \\
-(n-j)^{\alpha}(n-j+3+3 \alpha)
\end{array}\right] \\
& +\frac{\alpha(\Delta t)^{\alpha}}{2 A B(\alpha) \Gamma(\alpha+3)} \sum_{j=2}^{n}\left[\begin{array}{c}
t_{j}^{1-\beta} g\left(t_{j}, y^{j}\right) \\
-2 t_{j-1}^{1-\beta} f\left(t_{j-1}, y^{j}-f\left(t_{j}, y^{j}\right) \Delta t\right) \\
+t_{j-2}^{1-\beta} f\left(\begin{array}{c}
t_{j-2}, y^{j}-\Delta t f\left(t_{j}, y^{j}\right) \\
-\Delta t f\left(t_{j-1}, y^{j}-f\left(t_{j}, y^{j}\right) \Delta t\right)
\end{array}\right)
\end{array}\right] \\
& \times\left[\begin{array}{c}
(n-j+1)^{\alpha}\left[\begin{array}{c}
2(n-j)^{2}+(3 \alpha+10)(n-j) \\
+2 \alpha^{2}+9 \alpha+12
\end{array}\right] \\
-(n-j)^{\alpha}\left[\begin{array}{c}
2(n-j)^{2}+(5 \alpha+10)(n-j) \\
+6 \alpha^{2}+18 \alpha+12
\end{array}\right]
\end{array}\right] .
\end{aligned}
$$

With the Caputo fractal-fractional derivative, we consider the following:

$$
\begin{aligned}
& { }_{0}^{F F P} D_{t}^{\alpha, \beta} y(t)=f(t, y(t)), \\
& y(0)=y_{0} .
\end{aligned}
$$

Applying the new fractional integral with power-law kernel, we transform the above equation into

$$
y(t)=y(0)+\frac{1}{\Gamma(\alpha)} \int_{0}^{t} f(\tau, y(\tau))(t-\tau)^{\alpha-1} \tau^{1-\beta} d \tau .
$$

At the point $t_{n+1}=(n+1) \Delta t$, we obtain the following:

$$
y\left(t_{n+1}\right)=y(0)+\frac{1}{\Gamma(\alpha)} \int_{0}^{t_{n+1}} f(\tau, y(\tau))\left(t_{n+1}-\tau\right)^{\alpha-1} \tau^{1-\beta} d \tau .
$$

For simplicity, we shall take

$$
F(t, y(t))=f(t, y(t)) t^{1-\beta} .
$$

We also have

$$
y\left(t_{n+1}\right)=y(0)+\frac{1}{\Gamma(\alpha)} \sum_{j=2}^{n} \int_{t_{j}}^{t_{j+1}} F(\tau, y(\tau))\left(t_{n+1}-\tau\right)^{\alpha-1} d \tau
$$


Replacing them into the above equation and substituting $F(t, y(t))=f(t, y(t)) t^{1-\beta}$, we can get the following numerical scheme:

$$
\begin{aligned}
& y^{n+1}=\frac{(\Delta t)^{\alpha}}{\Gamma(\alpha+1)} \sum_{j=2}^{n} t_{j-2}^{1-\beta} f\left(\begin{array}{c}
t_{j-2}, y^{j}-\Delta t f\left(t_{j}, y^{j}\right) \\
-\Delta t f\left(t_{j-1}, y^{j}-f\left(t_{j}, y^{j}\right) \Delta t\right)
\end{array}\right) \\
& \times\left[(n-j+1)^{\alpha}-(n-j)^{\alpha}\right] \\
& +\frac{(\Delta t)^{\alpha}}{\Gamma(\alpha+2)} \sum_{j=2}^{n}\left[\begin{array}{c}
t_{j-1}^{1-\beta} f\left(t_{j-1}, y^{j}-f\left(t_{j}, y^{j}\right) \Delta t\right) \\
-t_{j-2}^{1-\beta} f\left(\begin{array}{c}
t_{j-2}, y^{j}-\Delta t f\left(t_{j}, y^{j}\right) \\
-\Delta t f\left(t_{j-1}, y^{j}-f\left(t_{j}, y^{j}\right) \Delta t\right)
\end{array}\right)
\end{array}\right] \\
& \times\left[\begin{array}{c}
(n-j+1)^{\alpha}(n-j+3+2 \alpha) \\
-(n-j)^{\alpha}(n-j+3+3 \alpha)
\end{array}\right] \\
& +\frac{(\Delta t)^{\alpha}}{2 \Gamma(\alpha+3)} \sum_{j=2}^{n}\left[\begin{array}{c}
t_{j}^{1-\beta} g\left(t_{j}, y^{j}\right) \\
-2 t_{j-1}^{1-\beta} f\left(t_{j-1}, y^{j}-f\left(t_{j}, y^{j}\right) \Delta t\right) \\
+t_{j-2}^{1-\beta} f\left(\begin{array}{c}
t_{j-2}, y^{j}-\Delta t f\left(t_{j}, y^{j}\right) \\
-\Delta t f\left(t_{j-1}, y^{j}-f\left(t_{j}, y^{j}\right) \Delta t\right)
\end{array}\right)
\end{array}\right] \\
& \times\left[\begin{array}{c}
(n-j+1)^{\alpha}\left[\begin{array}{c}
2(n-j)^{2}+(3 \alpha+10)(n-j) \\
+2 \alpha^{2}+9 \alpha+12
\end{array}\right] \\
-(n-j)^{\alpha}\left[\begin{array}{c}
2(n-j)^{2}+(5 \alpha+10)(n-j) \\
+6 \alpha^{2}+18 \alpha+12
\end{array}\right]
\end{array}\right] .
\end{aligned}
$$

Finally, we present the numerical scheme with fractal-fractional derivative with variable order. We start with the Caputo-Fabrizio case:

$$
\begin{aligned}
& { }_{0}^{F F E} D_{t}^{\alpha, \beta(t)} y(t)=f(t, y(t)), \\
& y(0)=y_{0} .
\end{aligned}
$$

The above equation can be reformulated as follows:

$$
\begin{aligned}
y(t)= & \frac{1-\alpha}{M(\alpha)} t^{2-\beta(t)}\left[-\beta \prime(t) \ln (t)+\frac{2-\beta(t)}{t}\right] f(t, y(t)) \\
& +\frac{\alpha}{M(\alpha)} \int_{0}^{t} f(\tau, y(\tau))\left[\beta \prime(\tau) \ln (\tau)+\frac{2-\beta(\tau)}{\tau}\right] \tau^{2-\beta(\tau)} d \tau .
\end{aligned}
$$

We write the above equation as follows:

$$
\begin{aligned}
y\left(t_{n+1}\right)-y\left(t_{n}\right)= & \frac{1-\alpha}{M(\alpha)}\left[\begin{array}{c}
t_{n+1}^{2-\beta\left(t_{n+1}\right)}\left(-\frac{\beta\left(t_{n+2}\right)-\beta\left(t_{n+1}\right)}{\Delta t} \ln t_{n+1}+\frac{2-\beta\left(t_{n+1}\right)}{t_{n+1}}\right) f\left(t_{n+1}, y\left(t_{n+1}\right)\right) \\
-t_{n}^{2-\beta\left(t_{n}\right)}\left(-\frac{\beta\left(t_{n+1}\right)-\beta\left(t_{n}\right)}{\Delta t} \ln t_{n}+\frac{2-\beta\left(t_{n}\right)}{t_{n}}\right) f\left(t_{n}, y\left(t_{n}\right)\right)
\end{array}\right] \\
& +\frac{\alpha}{M(\alpha)} \int_{t_{n}}^{t_{n+1}} f(\tau, y(\tau))\left[\beta \prime(\tau) \ln (\tau)+\frac{2-\beta(\tau)}{\tau}\right] \tau^{2-\beta(\tau)} d \tau .
\end{aligned}
$$

For simplicity, we take

$$
F(t, y(t))=f(t, y(t))\left[-\beta \prime(t) \ln (t)+\frac{2-\beta(t)}{t}\right] t^{2-\beta(t)}
$$


and we have

$$
\begin{aligned}
y\left(t_{n+1}\right)-y\left(t_{n}\right)= & \frac{1-\alpha}{M(\alpha)}\left[F\left(t_{n+1}, y\left(t_{n+1}\right)\right)-F\left(t_{n}, y\left(t_{n}\right)\right)\right] \\
& +\frac{\alpha}{M(\alpha)} \int_{t_{n}}^{t_{n+1}} F(\tau, y(\tau)) d \tau .
\end{aligned}
$$

If we do the same routine and replace $F(t, y(t))$ with its value, we have the following numerical approximation:

$$
y^{n+1}=y^{n}+\frac{1-\alpha}{M(\alpha)}\left[\begin{array}{c}
t_{n+1}^{2-\beta\left(t_{n+1}\right)}\left(-\frac{\beta\left(t_{n+2}\right)-\beta\left(t_{n+1}\right)}{\Delta t} \ln t_{n+1}+\frac{2-\beta\left(t_{n+1}\right)}{t_{n+1}}\right) \\
\times f\left(t_{n+1}, y\left(t_{n}\right)-\Delta t f\left(t_{n}, y\left(t_{n}\right)\right)\right) \\
-t_{n}^{2-\beta\left(t_{n}\right)}\left(-\frac{\beta\left(t_{n+1}\right)-\beta\left(t_{n}\right)}{\Delta t} \ln t_{n}+\frac{2-\beta\left(t_{n}\right)}{t_{n}}\right) \\
\times f\left(t_{n}, y\left(t_{n}\right)\right)
\end{array}\right]+\frac{\alpha}{M(\alpha)}\left\{\begin{array}{c}
\frac{23}{12} t_{n}^{2-\beta\left(t_{n}\right)}\left(-\frac{\beta\left(t_{n+1}\right)-\beta\left(t_{n}\right)}{\Delta t} \ln t_{n}+\frac{2-\beta\left(t_{n}\right)}{t_{n}}\right) \\
\times \frac{23}{12} f\left(t_{n}, y^{n}\right) \Delta t \\
-\frac{4}{3} t_{n-1}^{2-\beta\left(t_{n-1}\right)}\left(-\frac{\beta\left(t_{n}\right)-\beta\left(t_{n-1}\right)}{\Delta t} \ln t_{n-1}+\frac{2-\beta\left(t_{n-1}\right)}{t_{n-1}}\right) \\
\times f\left(t_{n-1}, y^{n}-f\left(t_{n}, y^{n}\right) \Delta t\right) \Delta t \\
+\frac{5}{12} t_{n-2}^{2-\beta\left(t_{n-2}\right)}\left(-\frac{\beta\left(t_{n-1}\right)-\beta\left(t_{n-2}\right)}{\Delta t} \ln t_{n-2}+\frac{2-\beta\left(t_{n-2}\right)}{t_{n-2}}\right) \\
\times \frac{5}{12} f\left(\begin{array}{c}
t_{n-2}, y^{n}-\Delta t f\left(t_{n}, y^{n}\right) \\
-\Delta t f\left(t_{n-1}, y^{n}-f\left(t_{n}, y^{n}\right) \Delta t\right)
\end{array}\right) \Delta t
\end{array}\right\} .
$$

We deal with our problem involving the new constant fractional order and variable fractal dimension

$$
\begin{aligned}
& { }_{0}^{F F M} D_{t}^{\alpha, \beta(t)} y(t)=f(t, y(t)), \\
& y(0)=y_{0},
\end{aligned}
$$

where the kernel is the Mittag-Leffler kernel. If we integrate the above equation with the new integral operator including the Mittag-Leffler kernel, the above equation can be converted to

$$
\begin{aligned}
y(t)= & \frac{1-\alpha}{A B(\alpha)} t^{2-\beta(t)}\left[-\beta \prime(t) \ln (t)+\frac{2-\beta(t)}{t}\right] f(t, y(t)) \\
& +\frac{\alpha}{A B(\alpha) \Gamma(\alpha)} \int_{0}^{t} f(\tau, y(\tau))(t-\tau)^{\alpha-1} \\
& \times\left[-\beta /(\tau) \ln (\tau)+\frac{2-\beta(\tau)}{\tau}\right] \tau^{2-\beta(\tau)} d \tau .
\end{aligned}
$$

At the point $t_{n+1}=(n+1) \Delta t$, we have the following:

$$
\begin{aligned}
y\left(t_{n+1}\right)= & \frac{1-\alpha}{A B(\alpha)} t_{n+1}^{2-\beta\left(t_{n+1}\right)}\left(-\frac{\beta\left(t_{n+2}\right)-\beta\left(t_{n+1}\right)}{\Delta t} \ln t_{n+1}+\frac{2-\beta\left(t_{n+1}\right)}{t_{n+1}}\right) \\
& \times f\left(t_{n+1}, y\left(t_{n+1}\right)\right)
\end{aligned}
$$




$$
\begin{aligned}
& +\frac{\alpha}{A B(\alpha) \Gamma(\alpha)} \int_{0}^{t_{n+1}} f(\tau, y(\tau))\left(t_{n+1}-s\right)^{\alpha-1} \\
& \times\left[-\beta \prime(\tau) \ln (\tau)+\frac{2-\beta(\tau)}{\tau}\right] \tau^{2-\beta(\tau)} d \tau .
\end{aligned}
$$

For brevity, we consider

$$
F(\tau, y(\tau))=f(\tau, y(\tau))\left[-\beta \prime(\tau) \ln (\tau)+\frac{2-\beta(\tau)}{\tau}\right] \tau^{2-\beta(\tau)},
$$

and we can write the following:

$$
\begin{aligned}
y\left(t_{n+1}\right)= & \frac{1-\alpha}{A B(\alpha)} t_{n+1}^{2-\beta\left(t_{n+1}\right)}\left(-\frac{\beta\left(t_{n+2}\right)-\beta\left(t_{n+1}\right)}{\Delta t} \ln t_{n+1}+\frac{2-\beta\left(t_{n+1}\right)}{t_{n+1}}\right) \\
& \times f\left(t_{n+1}, y\left(t_{n+1}\right)\right) \\
& +\frac{\alpha}{A B(\alpha) \Gamma(\alpha)} \sum_{j=2}^{n} \int_{t_{j}}^{t_{j+1}} F(\tau, y(\tau))\left(t_{n+1}-\tau\right)^{\alpha-1} d \tau .
\end{aligned}
$$

One can replace the Newton polynomial in the above equation as follows. Thus, we have the following scheme:

$$
\begin{aligned}
y^{n+1}= & \frac{1-\alpha}{A B(\alpha)} t_{n+1}^{2-\beta\left(t_{n+1}\right)}\left(-\frac{\beta\left(t_{n+2}\right)-\beta\left(t_{n+1}\right)}{\Delta t} \ln t_{n+1}+\frac{2-\beta\left(t_{n+1}\right)}{t_{n+1}}\right) \\
& \times f\left(t_{n+1}, y\left(t_{n+1}\right)\right) \\
& +\frac{\alpha(\Delta t)^{\alpha}}{A B(\alpha) \Gamma(\alpha+1)} \sum_{j=2}^{n} F\left(t_{j-2}, y^{j-2}\right)\left[(n-j+1)^{\alpha}-(n-j)^{\alpha}\right] \\
& +\frac{\alpha(\Delta t)^{\alpha}}{A B(\alpha) \Gamma(\alpha+2)} \sum_{j=2}^{n}\left[F\left(t_{j-1}, y^{j-1}\right)-F\left(t_{j-2}, y^{j-2}\right)\right] \\
& \times\left[\begin{array}{c}
(n-j+1)^{\alpha}(n-j+3+2 \alpha) \\
-(n-j)^{\alpha}(n-j+3+3 \alpha)
\end{array}\right] \\
& +\frac{\alpha(\Delta t)^{\alpha}}{2 A B(\alpha) \Gamma(\alpha+3)} \sum_{j=2}^{n}\left[F\left(t_{j}, y^{j}\right)-2 F\left(t_{j-1}, y^{j-1}\right)+F\left(t_{j-2}, y^{j-2}\right)\right] \\
& \times\left[\begin{array}{c}
(n-j+1)^{\alpha}\left[\begin{array}{l}
2(n-j)^{2}+(3 \alpha+10)(n-j) \\
+2 \alpha^{2}+9 \alpha+12
\end{array}\right] \\
-(n-j)^{\alpha}\left[\begin{array}{l}
2(n-j)^{2}+(5 \alpha+10)(n-j) \\
+6 \alpha^{2}+18 \alpha+12
\end{array}\right]
\end{array}\right] .
\end{aligned}
$$

Replacing the function $G(t, y(t))$ with its value, we can present the following scheme for numerical solution of our equation:

$$
\begin{aligned}
y^{n+1}= & \frac{1-\alpha}{A B(\alpha)} t_{n+1}^{2-\beta\left(t_{n+1}\right)}\left(-\frac{\beta\left(t_{n+2}\right)-\beta\left(t_{n+1}\right)}{\Delta t} \ln t_{n+1}+\frac{2-\beta\left(t_{n+1}\right)}{t_{n+1}}\right) \\
& \times f\left(t_{n+1}, y^{n}+f\left(t_{n}, y^{n}\right) \Delta t\right)
\end{aligned}
$$




$$
\begin{aligned}
& +\frac{\alpha(\Delta t)^{\alpha}}{A B(\alpha) \Gamma(\alpha+1)} \sum_{j=2}^{n} t_{j-2}^{2-\beta\left(t_{j-2}\right)}\left[-\frac{\beta\left(t_{j-1}\right)-\beta\left(t_{j-2}\right)}{\Delta t} \ln t_{j-2}+\frac{2-\beta\left(t_{j-2}\right)}{t_{j-2}}\right] \\
& \times f\left(\begin{array}{c}
t_{j-2}, y^{j}-\Delta t f\left(t_{j}, y^{j}\right) \\
-\Delta t f\left(t_{j-1}, y^{j}-f\left(t_{j}, y^{j}\right) \Delta t\right)
\end{array}\right)\left[(n-j+1)^{\alpha}-(n-j)^{\alpha}\right] \\
& +\frac{\alpha(\Delta t)^{\alpha}}{A B(\alpha) \Gamma(\alpha+2)} \sum_{j=2}^{n}\left[\begin{array}{c}
t_{j-1}^{2-\beta\left(t_{j-1}\right)}\left[-\frac{\beta\left(t_{j}\right)-\beta\left(t_{j-1}\right)}{\Delta t} \ln t_{j-1}+\frac{2-\beta\left(t_{j-1}\right)}{t_{j-1}}\right] \\
\times f\left(t_{j-1}, y^{j}-f\left(t_{j}, y^{j}\right) \Delta t\right) \\
-t_{j-2}^{2-\beta\left(t_{j-2}\right)}\left[-\frac{\beta\left(t_{j-1}\right)-\beta\left(t_{j-2}\right)}{\Delta t} \ln t_{j-2}+\frac{2-\beta\left(t_{j-2}\right)}{t_{j-2}}\right] \\
\times f\left(\begin{array}{c}
t_{j-2}, y^{j}-\Delta t f\left(t_{j}, y^{j}\right) \\
-\Delta t f\left(t_{j-1}, y^{j}-f\left(t_{j}, y^{j}\right) \Delta t\right)
\end{array}\right)
\end{array}\right] \\
& \times\left[\begin{array}{c}
(n-j+1)^{\alpha}(n-j+3+2 \alpha) \\
-(n-j)^{\alpha}(n-j+3+3 \alpha)
\end{array}\right]
\end{aligned}
$$

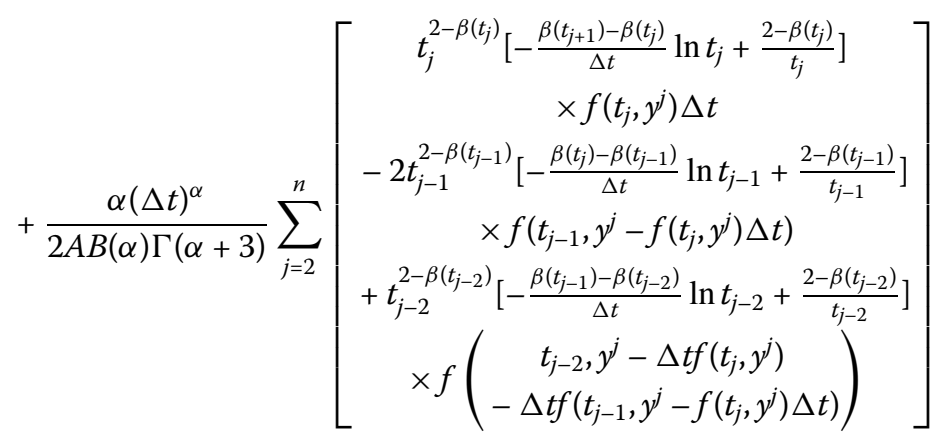

$$
\begin{aligned}
& \times\left[\begin{array}{c}
(n-j+1)^{\alpha}\left[\begin{array}{c}
2(n-j)^{2}+(3 \alpha+10)(n-j) \\
+2 \alpha^{2}+9 \alpha+12
\end{array}\right] \\
-(n-j)^{\alpha}\left[\begin{array}{c}
2(n-j)^{2}+(5 \alpha+10)(n-j) \\
+6 \alpha^{2}+18 \alpha+12
\end{array}\right]
\end{array}\right] .
\end{aligned}
$$

We deal with our problem involving the new constant fractional order and variable fractal dimension

$$
\begin{aligned}
& { }_{0}^{F F P} D_{t}^{\alpha, \beta(t)} y(t)=f(t, y(t)), \\
& y(0)=y_{0}
\end{aligned}
$$

where the kernel is the power-law kernel. If we integrate equation (103) with the new integral operator including the power-law kernel, the above equation can be converted to

$$
y(t)=\frac{1}{\Gamma(\alpha)} \int_{0}^{t} f(\tau, y(\tau))(t-\tau)^{\alpha-1}\left[-\beta \prime(\tau) \ln (\tau)+\frac{2-\beta(\tau)}{\tau}\right] \tau^{2-\beta(\tau)} d \tau .
$$

At the point $t_{n+1}=(n+1) \Delta t$, we have the following:

$$
\begin{aligned}
y\left(t_{n+1}\right)= & \frac{1}{\Gamma(\alpha)} \int_{0}^{t_{n+1}} f(\tau, y(\tau))\left(t_{n+1}-s\right)^{\alpha-1} \\
& \times\left[-\beta \prime(\tau) \ln (\tau)+\frac{2-\beta(\tau)}{\tau}\right] \tau^{2-\beta(\tau)} d \tau .
\end{aligned}
$$


For brevity, we consider

$$
F(\tau, y(\tau))=f(\tau, y(\tau))\left[-\beta \prime(\tau) \ln (\tau)+\frac{2-\beta(\tau)}{\tau}\right] \tau^{2-\beta(\tau)},
$$

and we can write the following:

$$
y\left(t_{n+1}\right)=\frac{1}{\Gamma(\alpha)} \sum_{j=2}^{n} \int_{t_{j}}^{t_{j+1}} F(\tau, y(\tau))\left(t_{n+1}-\tau\right)^{\alpha-1} d \tau .
$$

Thus, we have the following scheme:

$$
\begin{aligned}
y^{n+1}= & \frac{(\Delta t)^{\alpha}}{\Gamma(\alpha+1)} \sum_{j=2}^{n} F\left(t_{j-2}, y^{j-2}\right)\left[(n-j+1)^{\alpha}-(n-j)^{\alpha}\right] \\
& +\frac{(\Delta t)^{\alpha}}{\Gamma(\alpha+2)} \sum_{j=2}^{n}\left[F\left(t_{j-1}, y^{j-1}\right)-F\left(t_{j-2}, y^{j-2}\right)\right] \\
& \times\left[\begin{array}{c}
(n-j+1)^{\alpha}(n-j+3+2 \alpha) \\
-(n-j)^{\alpha}(n-j+3+3 \alpha)
\end{array}\right] \\
& +\frac{(\Delta t)^{\alpha}}{2 \Gamma(\alpha+3)} \sum_{j=2}^{n}\left[F\left(t_{j}, y^{j}\right)-2 F\left(t_{j-1}, y^{j-1}\right)+F\left(t_{j-2}, y^{j-2}\right)\right] \\
& \times\left[\begin{array}{c}
(n-j+1)^{\alpha}\left[\begin{array}{c}
2(n-j)^{2}+(3 \alpha+10)(n-j) \\
+2 \alpha^{2}+9 \alpha+12
\end{array}\right] \\
-(n-j)^{\alpha}\left[\begin{array}{c}
2(n-j)^{2}+(5 \alpha+10)(n-j) \\
+6 \alpha^{2}+18 \alpha+12
\end{array}\right]
\end{array}\right] .
\end{aligned}
$$

Replacing the function $G(t, y(t))$ with its value, we can present the following scheme for numerical solution of our equation:

$$
\begin{aligned}
y^{n+1}= & \frac{(\Delta t)^{\alpha}}{\Gamma(\alpha+1)} \sum_{j=2}^{n} t_{j-2}^{2-\beta\left(t_{j-2}\right)}\left[-\frac{\beta\left(t_{j-1}\right)-\beta\left(t_{j-2}\right)}{\Delta t} \ln t_{j-2}+\frac{2-\beta\left(t_{j-2}\right)}{t_{j-2}}\right] \\
& \times f\left(\begin{array}{c}
t_{j-2}, y^{j}-\Delta t f\left(t_{j}, y^{j}\right) \\
-\Delta t f\left(t_{j-1}, y^{j}-f\left(t_{j}, y^{j}\right) \Delta t\right)
\end{array}\right)\left[(n-j+1)^{\alpha}-(n-j)^{\alpha}\right] \\
& +\frac{(\Delta t)^{\alpha}}{\Gamma(\alpha+2)} \sum_{j=2}^{n}\left[\begin{array}{c}
t_{j-1}^{2-\beta\left(t_{j-1}\right)}\left[-\frac{\beta\left(t_{j}\right)-\beta\left(t_{j-1}\right)}{\Delta t} \ln t_{j-1}+\frac{2-\beta\left(t_{j-1}\right)}{t_{j-1}}\right] \\
-t_{j-2}^{2-\beta\left(t_{j-2}\right)}\left[-\frac{\beta\left(t_{j-1}\right)-\beta\left(t_{j-2}\right)}{\Delta t} \ln t_{j-2}+\frac{2-\beta\left(t_{j-2}\right)}{t_{j-2}}\right] \\
\times f\left(\begin{array}{c}
t_{j-2}, y^{j}-\Delta t f\left(t_{j}, y^{j}\right) \\
-\Delta t f\left(t_{j-1}, y^{j}-f\left(t_{j}, y^{j}\right) \Delta t\right)
\end{array}\right)
\end{array}\right] \\
& \times\left[\begin{array}{c}
(n-j+1)^{\alpha}(n-j+3+2 \alpha) \\
-(n-j)^{\alpha}(n-j+3+3 \alpha)
\end{array}\right]
\end{aligned}
$$




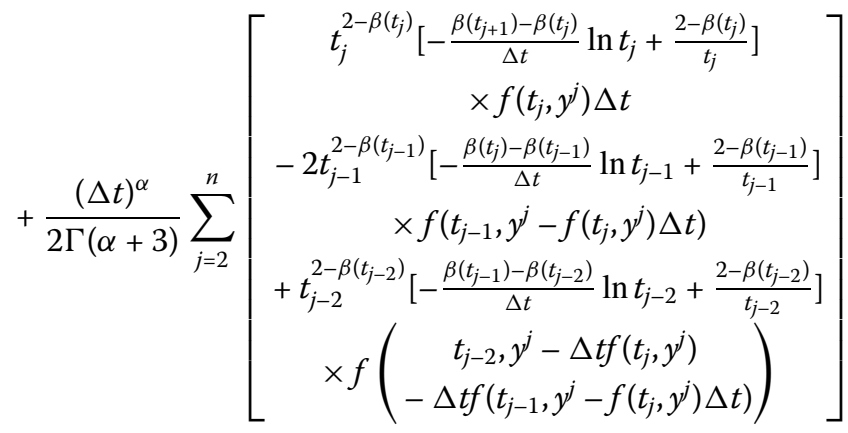

$$
\begin{aligned}
& \times\left[\begin{array}{c}
(n-j+1)^{\alpha}\left[\begin{array}{c}
2(n-j)^{2}+(3 \alpha+10)(n-j) \\
+2 \alpha^{2}+9 \alpha+12
\end{array}\right] \\
-(n-j)^{\alpha}\left[\begin{array}{c}
2(n-j)^{2}+(5 \alpha+10)(n-j) \\
+6 \alpha^{2}+18 \alpha+12
\end{array}\right]
\end{array}\right] .
\end{aligned}
$$

\section{Application to COVID-19 model}

In this section, using the suggested numerical scheme, we present its application to solve the mathematical model of COVID-19 with possibility of waves. The numerical scheme will be applied for all cases where the differential operators are with classical differential operators, modern fractional differential operators, and variable orders, although only few examples will be used for numerical simulations. Firstly, we shall use the Caputo-Fabrizio fractional derivative

$$
\begin{aligned}
& { }_{0}^{C F} D_{t}^{\alpha} S=\Lambda-\left(\delta(t)\left(\alpha I^{*}+w \beta I_{D}^{*}+\gamma w I_{A}^{*}+w \delta_{1} I_{R}^{*}+w \delta_{2} I_{T}^{*}\right)+\gamma_{1}+\mu_{1}\right) S, \\
& { }_{0}^{C F} D_{t}^{\alpha} I=\left(\delta(t)\left(\alpha I^{*}+w \beta I_{D}^{*}+\gamma w I_{A}^{*}+w \delta_{1} I_{R}^{*}+w \delta_{2} I_{T}^{*}\right)\right) S-\left(\varepsilon+\xi+\lambda+\mu_{1}\right) I, \\
& { }_{0}^{C F} D_{t}^{\alpha} I_{A}=\xi I-\left(\theta+\mu+\chi+\mu_{1}\right) I_{A}, \\
& { }_{0}^{C F} D_{t}^{\alpha} I_{D}=\varepsilon I-\left(\eta+\varphi+\mu_{1}\right) I_{D}, \\
& { }_{0}^{C F} D_{t}^{\alpha} I_{R}=\eta I_{D}+\theta I_{A}-\left(v+\xi+\mu_{1}\right) I_{R}, \\
& { }_{0}^{C F} D_{t}^{\alpha} I_{T}=\mu I_{A}+v I_{R}-\left(\sigma+\tau+\mu_{1}\right) I_{T}, \\
& { }_{0}^{C F} D_{t}^{\alpha} R=\lambda I+\varphi I_{D}+\chi I_{A}+\xi I_{R}+\sigma I_{T}-\left(\Phi+\mu_{1}\right) R, \\
& { }_{0}^{C F} D_{t}^{\alpha} D=\tau I_{T}, \\
& { }_{0}^{C F} D_{t}^{\alpha} V=\gamma_{1} S+\Phi R-\mu_{1} V .
\end{aligned}
$$

For simplicity, we rearrange the above equation as follows:

$$
\begin{aligned}
& { }_{0}^{C F} D_{t}^{\alpha} S=S^{*}\left(t, S, I, I_{A}, I_{D}, I_{R}, I_{T}, R, D, V\right), \\
& { }_{0}^{C F} D_{t}^{\alpha} I=I^{*}\left(t, S, I, I_{A}, I_{D}, I_{R}, I_{T}, R, D, V\right), \\
& { }_{0}^{C F} D_{t}^{\alpha} I_{A}=I_{A}^{*}\left(t, S, I, I_{A}, I_{D}, I_{R}, I_{T}, R, D, V\right), \\
& { }_{0}^{C F} D_{t}^{\alpha} I_{D}=I_{D}^{*}\left(t, S, I, I_{A}, I_{D}, I_{R}, I_{T}, R, D, V\right), \\
& { }_{0}^{C F} D_{t}^{\alpha} I_{R}=I_{R}^{*}\left(t, S, I, I_{A}, I_{D}, I_{R}, I_{T}, R, D, V\right), \\
& { }_{0}^{C F} D_{t}^{\alpha} I_{T}=I_{T}^{*}\left(t, S, I, I_{A}, I_{D}, I_{R}, I_{T}, R, D, V\right),
\end{aligned}
$$




$$
\begin{aligned}
& { }_{0}^{C F} D_{t}^{\alpha} R=R^{*}\left(t, S, I, I_{A}, I_{D}, I_{R}, I_{T}, R, D, V\right), \\
& { }_{0}^{C F} D_{t}^{\alpha} D=D^{*}\left(t, S, I, I_{A}, I_{D}, I_{R}, I_{T}, R, D, V\right), \\
& { }_{0}^{C F} D_{t}^{\alpha} V=V^{*}\left(t, S, I, I_{A}, I_{D}, I_{R}, I_{T}, R, D, V\right) .
\end{aligned}
$$

Thus, we can have the following scheme for our model:

$$
\begin{aligned}
& S^{n+1}=S^{n}+\frac{1-\alpha}{M(\alpha)}\left[\begin{array}{c}
S^{*}\left(\begin{array}{c}
t_{n+1}, S^{n}+\Delta t S^{n *}, I^{n}+\Delta t I^{n *}, I_{A}^{n}+\Delta t I_{A}^{n *}, \\
I_{D}^{n}+\Delta t I_{D}^{n *}, I_{R}^{n}+\Delta t I_{R}^{n *}, I_{T}^{n}+\Delta t I_{T}^{n *}, \\
R^{n}+\Delta t R^{n *}, D^{n}+\Delta t D^{n *}, V^{n}+\Delta t V^{n *}
\end{array}\right) \\
-S^{*}\left(t_{n}, S^{n}, I^{n}, I_{A}^{n}, I_{D}^{n}, I_{R}^{n}, I_{T}^{n}, R^{n}, D^{n}, V^{n}\right)
\end{array}\right] \\
& +\frac{\alpha}{M(\alpha)}\left\{\begin{array}{c}
\frac{23}{12} S^{*}\left(t_{n}, S^{n}, I^{n}, I_{A}^{n}, I_{D}^{n}, I_{R}^{n}, I_{T}^{n}, R^{n}, D^{n}, V^{n}\right) \Delta t \\
-\frac{4}{3} S^{*}\left(\begin{array}{c}
t_{n}, S^{n}-\Delta t S^{n *}, I^{n}-\Delta t I^{n *}, I_{A}^{n}-\Delta t I_{A}^{n *}, \\
I_{D}^{n}-\Delta t I_{D}^{n *}, I_{R}^{n}-\Delta t I_{R}^{n *}, I_{T}^{n}-\Delta t I_{T}^{n *}, \\
R^{n}-\Delta t R^{n *}, D^{n}-\Delta t D^{n *}, V^{n}-\Delta t V^{n *}
\end{array}\right) \Delta t \\
+\frac{5}{12} S^{*}\left(\begin{array}{c}
t_{n-2}, S^{n}-\Delta t S^{n *}-\Delta t S^{(n-1) *}, I^{n}-\Delta t I^{n *}-\Delta t I^{(n-1) *}, \\
I_{A}^{n}-\Delta t I_{A}^{n *}-\Delta t I_{A}^{(n-1) *}, I_{D}^{n}-\Delta t I_{D}^{n *}-\Delta t I_{D}^{(n-1) *}, \\
I_{R}^{n}-\Delta t I_{R}^{n *}-\Delta t I_{R}^{(n-1) *}, I_{T}^{n}-\Delta t I_{T}^{n *}-\Delta t I_{T}^{(n-1) *}, \\
R^{n}-\Delta t R^{n *}-\Delta t R^{(n-1) *}, D^{n}-\Delta t D^{n *}-\Delta t D^{(n-1) *}, \\
V^{n}-\Delta t V^{n *}-\Delta t V^{(n-1) *}
\end{array}\right.
\end{array}\right\}, \Delta t
\end{aligned}
$$

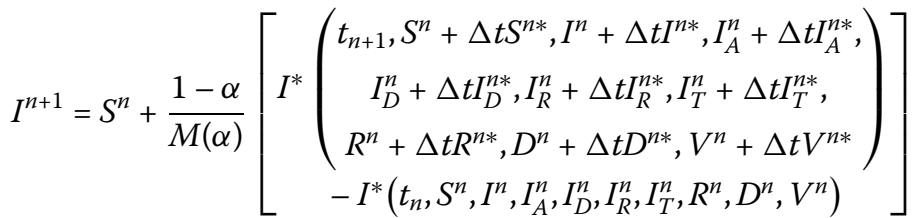

$$
\begin{aligned}
& +\frac{\alpha}{M(\alpha)}\left\{\begin{array}{c}
\frac{23}{12} I^{*}\left(t_{n}, S^{n}, I^{n}, I_{A}^{n}, I_{D}^{n}, I_{R}^{n}, I_{T}^{n}, R^{n}, D^{n}, V^{n}\right) \Delta t \\
-\frac{4}{3} I^{*}\left(\begin{array}{c}
t_{n}, S^{n}-\Delta t S^{n *}, I^{n}-\Delta t I^{n *}, I_{A}^{n}-\Delta t I_{A}^{n *}, \\
I_{D}^{n}-\Delta t I_{D}^{n *}, I_{R}^{n}-\Delta t I_{R}^{n *}, I_{T}^{n}-\Delta t I_{T}^{n *}, \\
R^{n}-\Delta t R^{n *}, D^{n}-\Delta t D^{n *}, V^{n}-\Delta t V^{n *}
\end{array}\right) \Delta t \\
+\frac{5}{12} I^{*}\left(\begin{array}{c}
t_{n-2}, S^{n}-\Delta t S^{n *}-\Delta t S^{(n-1) *}, I^{n}-\Delta t I^{n *}-\Delta t I^{(n-1) *}, \\
I_{A}^{n}-\Delta t I_{A}^{n *}-\Delta t I_{A}^{(n-1) *}, I_{D}^{n}-\Delta t I_{D}^{n *}-\Delta t I_{D}^{(n-1) *}, \\
I_{R}^{n}-\Delta t I_{R}^{n *}-\Delta t I_{R}^{(n-1) *}, I_{T}^{n}-\Delta t I_{T}^{n *}-\Delta t I_{T}^{(n-1) *}, \\
R^{n}-\Delta t R^{n *}-\Delta t R^{(n-1) *}, D^{n}-\Delta t D^{n *}-\Delta t D^{(n-1) *}, \\
V^{n}-\Delta t V^{n *}-\Delta t V^{(n-1) *}
\end{array}\right\} \Delta t
\end{array}\right\},
\end{aligned}
$$

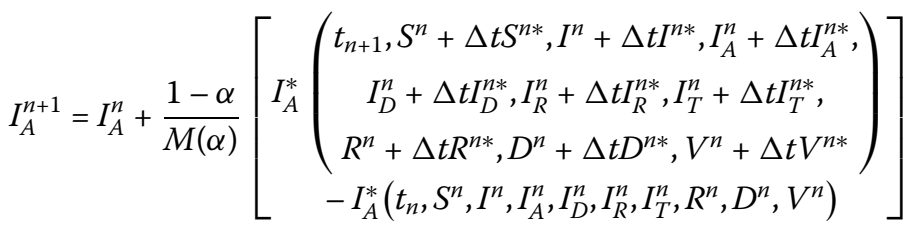




$$
\begin{aligned}
& +\frac{\alpha}{M(\alpha)}\left\{\begin{array}{c}
\frac{23}{12} I_{A}^{*}\left(t_{n}, S^{n}, I^{n}, I_{A}^{n}, I_{D}^{n}, I_{R}^{n}, I_{T}^{n}, R^{n}, D^{n}, V^{n}\right) \Delta t \\
-\frac{4}{3} I_{A}^{*}\left(\begin{array}{c}
t_{n}, S^{n}-\Delta t S^{n *}, I^{n}-\Delta t I^{n *}, I_{A}^{n}-\Delta t I_{A}^{n *}, \\
I_{D}^{n}-\Delta t I_{D}^{n *}, I_{R}^{n}-\Delta t I_{R}^{n *}, I_{T}^{n}-\Delta t I_{T}^{n *}, \\
R^{n}-\Delta t R^{n *}, D^{n}-\Delta t D^{n *}, V^{n}-\Delta t V^{n *}
\end{array}\right) \Delta t \\
+\frac{5}{12} I_{A}^{*}\left(\begin{array}{c}
t_{n-2}, S^{n}-\Delta t S^{n *}-\Delta t S^{(n-1) *}, I^{n}-\Delta t I^{n *}-\Delta t I^{(n-1) *}, \\
I_{A}^{n}-\Delta t I_{A}^{n *}-\Delta t I_{A}^{(n-1) *}, I_{D}^{n}-\Delta t I_{D}^{n *}-\Delta t I_{D}^{(n-1) *}, \\
I_{R}^{n}-\Delta t I_{R}^{n *}-\Delta t I_{R}^{(n-1) *}, I_{T}^{n}-\Delta t I_{T}^{n *}-\Delta t I_{T}^{(n-1) *}, \\
R^{n}-\Delta t R^{n *}-\Delta t R^{(n-1) *}, D^{n}-\Delta t D^{n *}-\Delta t D^{(n-1) *}, \\
V^{n}-\Delta t V^{n *}-\Delta t V^{(n-1) *}
\end{array}\right.
\end{array}\right\}, \Delta t \\
& I_{D}^{n+1}=I_{D}^{n}+\frac{1-\alpha}{M(\alpha)}\left[I_{D}^{*}\left(\begin{array}{c}
t_{n+1}, S^{n}+\Delta t S^{n *}, I^{n}+\Delta t I^{n *}, I_{A}^{n}+\Delta t I_{A}^{n *}, \\
I_{D}^{n}+\Delta t I_{D}^{n *}, I_{R}^{n}+\Delta t I_{R}^{n *}, I_{T}^{n}+\Delta t I_{T}^{n *}, \\
R^{n}+\Delta t R^{n *}, D^{n}+\Delta t D^{n *}, V^{n}+\Delta t V^{n *} \\
-I_{D}^{*}\left(t_{n}, S^{n}, I^{n}, I_{A}^{n}, I_{D}^{n}, I_{R}^{n}, I_{T}^{n}, R^{n}, D^{n}, V^{n}\right)
\end{array}\right]\right. \\
& +\frac{\alpha}{M(\alpha)}\left\{\begin{array}{c}
\frac{23}{12} I_{D}^{*}\left(t_{n}, S^{n}, I^{n}, I_{A}^{n}, I_{D}^{n}, I_{R}^{n}, I_{T}^{n}, R^{n}, D^{n}, V^{n}\right) \Delta t \\
-\frac{4}{3} I_{D}^{*}\left(\begin{array}{c}
t_{n}, S^{n}-\Delta t S^{n *}, I^{n}-\Delta t I^{n *}, I_{A}^{n}-\Delta t I_{A}^{n *}, \\
I_{D}^{n}-\Delta t I_{D}^{n *}, I_{R}^{n}-\Delta t I_{R}^{n *}, I_{T}^{n}-\Delta t I_{T}^{n *}, \\
R^{n}-\Delta t R^{n *}, D^{n}-\Delta t D^{n *}, V^{n}-\Delta t V^{n *}
\end{array}\right) \Delta t \\
+\frac{5}{12} I_{D}^{*}\left(\begin{array}{c}
t_{n-2}, S^{n}-\Delta t S^{n *}-\Delta t S^{(n-1) *}, I^{n}-\Delta t I^{n *}-\Delta t I^{(n-1) *}, \\
I_{A}^{n}-\Delta t I_{A}^{n *}-\Delta t I_{A}^{(n-1) *}, I_{D}^{n}-\Delta t I_{D}^{n *}-\Delta t I_{D}^{(n-1) *}, \\
I_{R}^{n}-\Delta t I_{R}^{n *}-\Delta t I_{R}^{(n-1) *}, I_{T}^{n}-\Delta t I_{T}^{n *}-\Delta t I_{T}^{(n-1) *}, \\
R^{n}-\Delta t R^{n *}-\Delta t R^{(n-1) *}, D^{n}-\Delta t D^{n *}-\Delta t D^{(n-1) *}, \\
V^{n}-\Delta t V^{n *}-\Delta t V^{(n-1) *}
\end{array}\right\} \Delta t
\end{array}\right\}, \\
& I_{R}^{n+1}=I_{R}^{n}+\frac{1-\alpha}{M(\alpha)}\left[\begin{array}{c}
I_{R}^{*}\left(\begin{array}{c}
t_{n+1}, S^{n}+\Delta t S^{n *}, I^{n}+\Delta t I^{n *}, I_{A}^{n}+\Delta t I_{A}^{n *}, \\
I_{D}^{n}+\Delta t I_{D}^{n *}, I_{R}^{n}+\Delta t I_{R}^{n *}, I_{T}^{n}+\Delta t I_{T}^{n *}, \\
R^{n}+\Delta t R^{n *}, D^{n}+\Delta t D^{n *}, V^{n}+\Delta t V^{n *}
\end{array}\right) \\
-I_{R}^{*}\left(t_{n}, S^{n}, I^{n}, I_{A}^{n}, I_{D}^{n}, I_{R}^{n}, I_{T}^{n}, R^{n}, D^{n}, V^{n}\right)
\end{array}\right] \\
& +\frac{\alpha}{M(\alpha)}\left\{\begin{array}{c}
\frac{23}{12} I_{R}^{*}\left(t_{n}, S^{n}, I^{n}, I_{A}^{n}, I_{D}^{n}, I_{R}^{n}, I_{T}^{n}, R^{n}, D^{n}, V^{n}\right) \Delta t \\
-\frac{4}{3} I_{R}^{*}\left(\begin{array}{c}
t_{n}, S^{n}-\Delta t S^{n *}, I^{n}-\Delta t I^{n *}, I_{A}^{n}-\Delta t I_{A}^{n *}, \\
I_{D}^{n}-\Delta t I_{D}^{n *}, I_{R}^{n}-\Delta t I_{R}^{n *}, I_{T}^{n}-\Delta t I_{T}^{n *}, \\
R^{n}-\Delta t R^{n *}, D^{n}-\Delta t D^{n *}, V^{n}-\Delta t V^{n *}
\end{array}\right) \Delta t \\
+\frac{5}{12} I_{R}^{*}\left(\begin{array}{c}
t_{n-2}, S^{n}-\Delta t S^{n *}-\Delta t S^{(n-1) *}, I^{n}-\Delta t I^{n *}-\Delta t I^{(n-1) *}, \\
I_{A}^{n}-\Delta t I_{A}^{n *}-\Delta t I_{A}^{(n-1) *}, I_{D}^{n}-\Delta t I_{D}^{n *}-\Delta t I_{D}^{(n-1) *}, \\
I_{R}^{n}-\Delta t I_{R}^{n *}-\Delta t I_{R}^{(n-1) *}, I_{T}^{n}-\Delta t I_{T}^{n *}-\Delta t I_{T}^{(n-1) *}, \\
R^{n}-\Delta t R^{n *}-\Delta t R^{(n-1) *}, D^{n}-\Delta t D^{n *}-\Delta t D^{(n-1) *}, \\
V^{n}-\Delta t V^{n *}-\Delta t V^{(n-1) *}
\end{array}\right) \Delta t
\end{array}\right\}, \\
& I_{T}^{n+1}=I_{T}^{n}+\frac{1-\alpha}{M(\alpha)}\left[I_{T}^{*}\left(\begin{array}{c}
t_{n+1}, S^{n}+\Delta t S^{n *}, I^{n}+\Delta t I^{n *}, I_{A}^{n}+\Delta t I_{A}^{n *}, \\
I_{D}^{n}+\Delta t I_{D}^{n *}, I_{R}^{n}+\Delta t I_{R}^{n *}, I_{T}^{n}+\Delta t I_{T}^{n *}, \\
R^{n}+\Delta t R^{n *}, D^{n}+\Delta t D^{n *}, V^{n}+\Delta t V^{n *} \\
-I_{T}^{*}\left(t_{n}, S^{n}, I^{n}, I_{A}^{n}, I_{D}^{n}, I_{R}^{n}, I_{T}^{n}, R^{n}, D^{n}, V^{n}\right)
\end{array}\right]\right.
\end{aligned}
$$




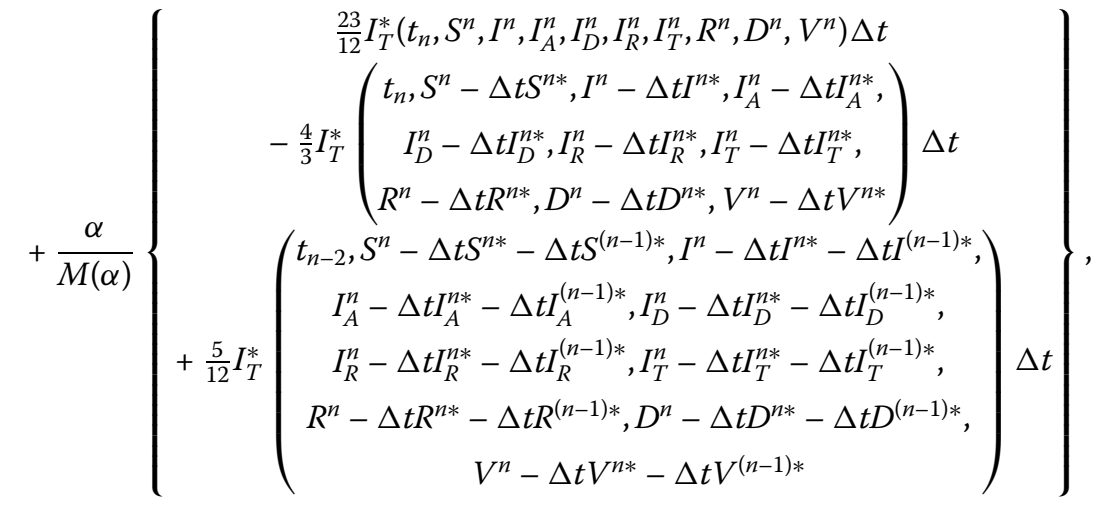

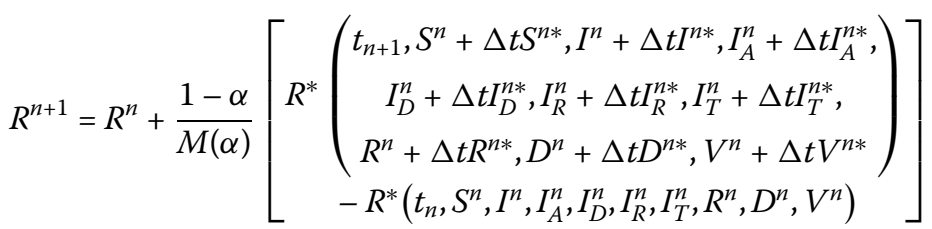

$$
\begin{aligned}
& +\frac{\alpha}{M(\alpha)}\left\{\begin{array}{c}
\frac{23}{12} R^{*}\left(t_{n}, S^{n}, I^{n}, I_{A}^{n}, I_{D}^{n}, I_{R}^{n}, I_{T}^{n}, R^{n}, D^{n}, V^{n}\right) \Delta t \\
-\frac{4}{3} R^{*}\left(\begin{array}{c}
t_{n}, S^{n}-\Delta t S^{n *}, I^{n}-\Delta t I^{n *}, I_{A}^{n}-\Delta t I_{A}^{n *}, \\
I_{D}^{n}-\Delta t I_{D}^{n *}, I_{R}^{n}-\Delta t I_{R}^{n *}, I_{T}^{n}-\Delta t I_{T}^{n *}, \\
R^{n}-\Delta t R^{n *}, D^{n}-\Delta t D^{n *}, V^{n}-\Delta t V^{n *}
\end{array}\right) \Delta t \\
+\frac{5}{12} R^{*}\left(\begin{array}{c}
t_{n-2}, S^{n}-\Delta t S^{n *}-\Delta t S^{(n-1) *}, I^{n}-\Delta t I^{n *}-\Delta t I^{(n-1) *}, \\
I_{A}^{n}-\Delta t I_{A}^{n *}-\Delta t I_{A}^{(n-1) *}, I_{D}^{n}-\Delta t I_{D}^{n *}-\Delta t I_{D}^{(n-1) *}, \\
I_{R}^{n}-\Delta t I_{R}^{n *}-\Delta t I_{R}^{(n-1) *}, I_{T}^{n}-\Delta t I_{T}^{n *}-\Delta t I_{T}^{(n-1) *}, \\
R^{n}-\Delta t R^{n *}-\Delta t R^{(n-1) *}, D^{n}-\Delta t D^{n *}-\Delta t D^{(n-1) *}, \\
V^{n}-\Delta t V^{n *}-\Delta t V^{(n-1) *}
\end{array}\right) \Delta t
\end{array}\right\},
\end{aligned}
$$

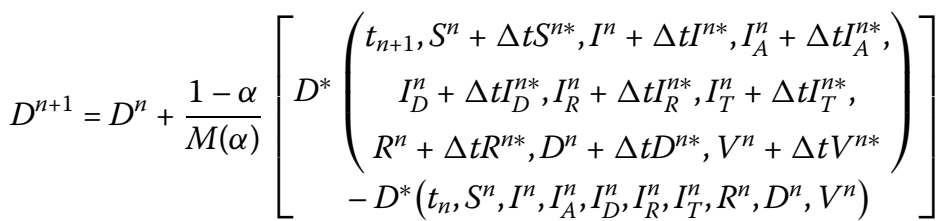

$$
\begin{aligned}
& +\frac{\alpha}{M(\alpha)}\left\{\begin{array}{c}
\frac{23}{12} D^{*}\left(t_{n}, S^{n}, I^{n}, I_{A}^{n}, I_{D}^{n}, I_{R}^{n}, I_{T}^{n}, R^{n}, D^{n}, V^{n}\right) \Delta t \\
-\frac{4}{3} D^{*}\left(\begin{array}{c}
t_{n}, S^{n}-\Delta t S^{n *}, I^{n}-\Delta t I^{n *}, I_{A}^{n}-\Delta t I_{A}^{n *}, \\
I_{D}^{n}-\Delta t I_{D}^{n *}, I_{R}^{n}-\Delta t I_{R}^{n *}, I_{T}^{n}-\Delta t I_{T}^{n *}, \\
R^{n}-\Delta t R^{n *}, D^{n}-\Delta t D^{n *}, V^{n}-\Delta t V^{n *}
\end{array}\right) \Delta t \\
+\frac{5}{12} D^{*}\left(\begin{array}{c}
t_{n-2}, S^{n}-\Delta t S^{n *}-\Delta t S^{(n-1) *}, I^{n}-\Delta t I^{n *}-\Delta t I^{(n-1) *}, \\
I_{A}^{n}-\Delta t I_{A}^{n *}-\Delta t I_{A}^{(n-1) *}, I_{D}^{n}-\Delta t I_{D}^{n *}-\Delta t I_{D}^{(n-1) *}, \\
I_{R}^{n}-\Delta t I_{R}^{n *}-\Delta t I_{R}^{(n-1) *}, I_{T}^{n}-\Delta t I_{T}^{n *}-\Delta t I_{T}^{(n-1) *}, \\
R^{n}-\Delta t R^{n *}-\Delta t R^{(n-1) *}, D^{n}-\Delta t D^{n *}-\Delta t D^{(n-1) *}, \\
V^{n}-\Delta t V^{n *}-\Delta t V^{(n-1) *}
\end{array}\right\} \Delta t
\end{array}\right\} \\
& V^{n+1}=V^{n}+\frac{1-\alpha}{M(\alpha)}\left[\begin{array}{c}
V^{*}\left(\begin{array}{c}
t_{n+1}, S^{n}+\Delta t S^{n *}, I^{n}+\Delta t I^{n *}, I_{A}^{n}+\Delta t I_{A}^{n *}, \\
I_{D}^{n}+\Delta t I_{D}^{n *}, I_{R}^{n}+\Delta t I_{R}^{n *}, I_{T}^{n}+\Delta t I_{T}^{n *}, \\
R^{n}+\Delta t R^{n *}, D^{n}+\Delta t D^{n *}, V^{n}+\Delta t V^{n *}
\end{array}\right) \\
-V^{*}\left(t_{n}, S^{n}, I^{n}, I_{A}^{n}, I_{D}^{n}, I_{R}^{n}, I_{T}^{n}, R^{n}, D^{n}, V^{n}\right)
\end{array}\right]
\end{aligned}
$$




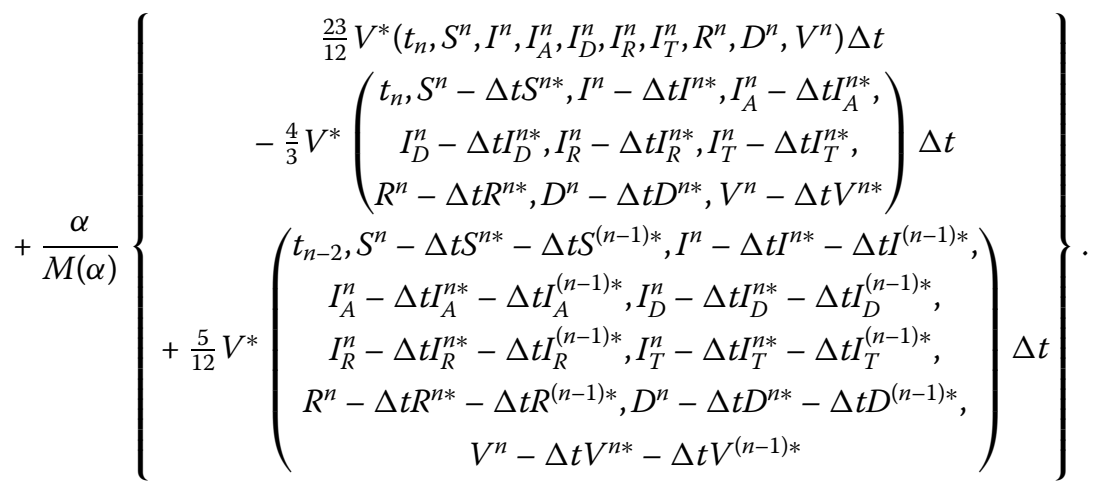

With the Atangana-Baleanu fractional derivative, we can solve numerically our model as follows:

$$
\begin{aligned}
& S^{n+1}=\frac{1-\alpha}{A B(\alpha)} S^{*}\left(\begin{array}{c}
t_{n+1}, S^{n}+\Delta t S^{n *}, I^{n}+\Delta t I^{n *}, I_{A}^{n}+\Delta t I_{A}^{n *}, \\
I_{D}^{n}+\Delta t I_{D}^{n *}, I_{R}^{n}+\Delta t I_{R}^{n *}, I_{T}^{n}+\Delta t I_{T}^{n *}, \\
R^{n}+\Delta t R^{n *}, D^{n}+\Delta t D^{n *}, V^{n}+\Delta t V^{n *}
\end{array}\right) \\
& +\frac{\alpha(\Delta t)^{\alpha}}{A B(\alpha) \Gamma(\alpha+1)} \\
& \times \sum_{j=2}^{n} S^{*}\left(\begin{array}{c}
t_{j-2}, S^{j}-\Delta t S^{j *}-\Delta t S^{(j-1) *}, I^{j}-\Delta t I^{j *}-\Delta t I^{(j-1) *}, \\
I_{A}^{j}-\Delta t I_{A}^{j *}-\Delta t I_{A}^{(j-1) *}, I_{D}^{j}-\Delta t I_{D}^{j *}-\Delta t I_{D}^{(j-1) *}, \\
I_{R}^{j}-\Delta t I_{R}^{j *}-\Delta t I_{R}^{(j-1) *}, I_{T}^{j}-\Delta t I_{T}^{j *}-\Delta t I_{T}^{(j-1) *}, \\
R^{j}-\Delta t R^{j *}-\Delta t R^{(j-1) *}, D^{j}-\Delta t D^{j *}-\Delta t D^{(j-1) *}, \\
V^{j}-\Delta t V^{j *}-\Delta t V^{(j-1) *}
\end{array}\right) \times \Pi \\
& +\frac{\alpha(\Delta t)^{\alpha}}{A B(\alpha) \Gamma(\alpha+2)}
\end{aligned}
$$

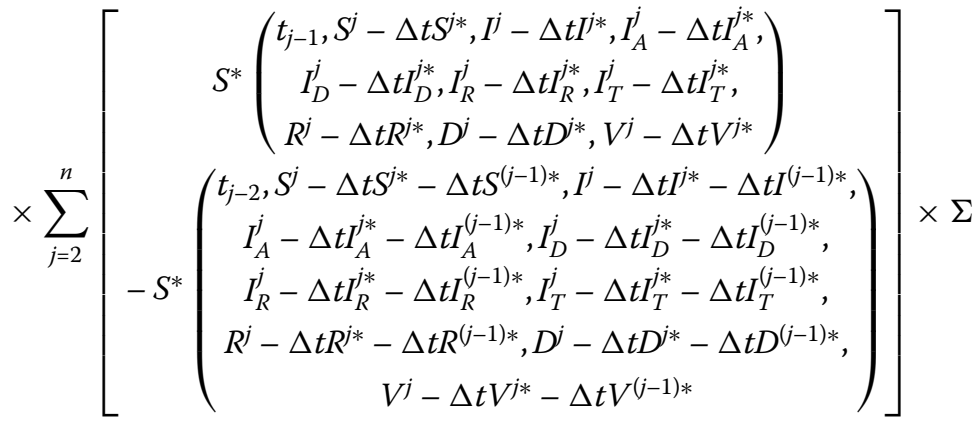

$$
\begin{aligned}
& +\frac{\alpha(\Delta t)^{\alpha}}{2 A B(\alpha) \Gamma(\alpha+3)} \\
& \left.\times \sum_{j=2}^{n}\left[\begin{array}{c}
S^{*}\left(t_{j}, S^{j}, I^{j}, I_{A}^{j}, I_{D}^{j}, I_{R}^{j}, I_{T}^{j}, R^{j}, D^{j}, V^{j}\right) \\
t_{j-1}, S^{j}-\Delta t S^{j *}, I^{j}-\Delta t I^{j *}, I_{A}^{j}-\Delta t I_{A}^{j *}, \\
-2 S^{*}\left(\begin{array}{c}
j \\
I_{D}^{j}-\Delta t I_{D}^{j *}, I_{R}^{j}-\Delta t I_{R}^{j *}, I_{T}^{j}-\Delta t I_{T}^{j *}, R^{j}-\Delta t R^{j *}, \\
D^{j}-\Delta t D^{j *}, V^{j}-\Delta t V^{j *}
\end{array}\right) \\
t_{j-2}, S^{j}-\Delta t S^{j *}-\Delta t S^{(j-1) *}, I^{j}-\Delta t I^{j *}-\Delta t I^{(j-1) *}, \\
I_{A}^{j}-\Delta t I_{A}^{j *}-\Delta t I_{A}^{(j-1) *}, I_{D}^{j}-\Delta t I_{D}^{j *}-\Delta t I_{D}^{(j-1) *}, \\
I_{R}^{j}-\Delta t I_{R}^{j *}-\Delta t I_{R}^{(j-1) *}, I_{T}^{j}-\Delta t I_{T}^{j *}-\Delta t I_{T}^{(j-1) *}, \\
R^{j}-\Delta t R^{j *}-\Delta t R^{(j-1) *}, D^{j}-\Delta t D^{j *}-\Delta t D^{(j-1) *}, \\
V^{j}-\Delta t V^{j *}-\Delta t V^{(j-1) *}
\end{array}\right)\right] \times \Delta,
\end{aligned}
$$




$$
\begin{aligned}
& I^{n+1}=\frac{1-\alpha}{A B(\alpha)} I^{*}\left(\begin{array}{c}
t_{n+1}, S^{n}+\Delta t S^{n *}, I^{n}+\Delta t I^{n *}, I_{A}^{n}+\Delta t I_{A}^{n *}, \\
I_{D}^{n}+\Delta t I_{D}^{n *}, I_{R}^{n}+\Delta t I_{R}^{n *}, I_{T}^{n}+\Delta t I_{T}^{n *}, \\
R^{n}+\Delta t R^{n *}, D^{n}+\Delta t D^{n *}, V^{n}+\Delta t V^{n *}
\end{array}\right) \\
& +\frac{\alpha(\Delta t)^{\alpha}}{A B(\alpha) \Gamma(\alpha+1)} \\
& \times \sum_{j=2}^{n} I^{*}\left(\begin{array}{c}
t_{j-2}, S^{j}-\Delta t S^{j *}-\Delta t S^{(j-1) *}, I^{j}-\Delta t I^{j *}-\Delta t I^{(j-1) *}, \\
I_{A}^{j}-\Delta t I_{A}^{j *}-\Delta t I_{A}^{(j-1) *}, I_{D}^{j}-\Delta t I_{D}^{j *}-\Delta t I_{D}^{(j-1) *}, \\
I_{R}^{j}-\Delta t I_{R}^{j *}-\Delta t I_{R}^{(j-1) *}, I_{T}^{j}-\Delta t I_{T}^{j *}-\Delta t I_{T}^{(j-1) *}, \\
R^{j}-\Delta t R^{j *}-\Delta t R^{(j-1) *}, D^{j}-\Delta t D^{j *}-\Delta t D^{(j-1) *}, \\
V^{j}-\Delta t V^{j *}-\Delta t V^{(j-1) *}
\end{array}\right) \times \Pi \\
& +\frac{\alpha(\Delta t)^{\alpha}}{A B(\alpha) \Gamma(\alpha+2)}
\end{aligned}
$$

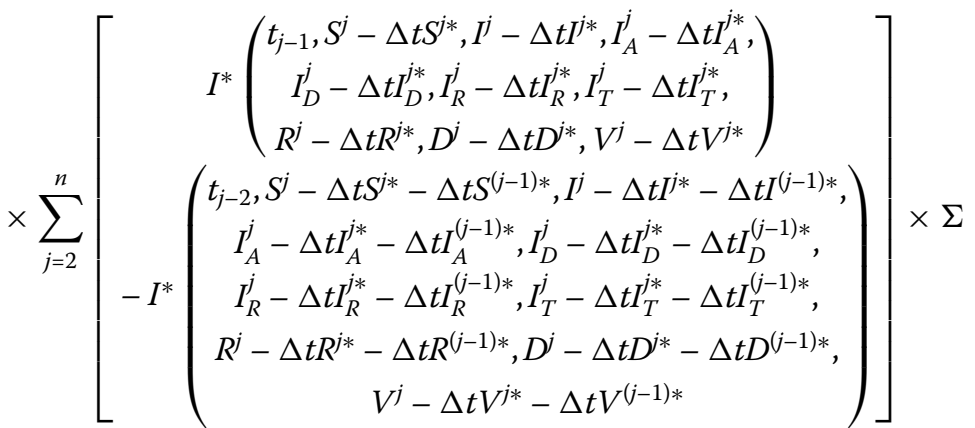

$$
\begin{aligned}
& +\frac{\alpha(\Delta t)^{\alpha}}{2 A B(\alpha) \Gamma(\alpha+3)} \\
& \times \sum_{j=2}^{n}\left[\begin{array}{c}
I^{*}\left(t_{j}, S^{j}, I^{j}, I_{A}^{j}, I_{D}^{j}, I_{R}^{j}, I_{T}^{j}, R^{j}, D^{j}, V^{j}\right) \\
-2 I^{*}\left(\begin{array}{c}
t_{j-1}, S^{j}-\Delta t S^{j *}, I^{j}-\Delta t I^{j *}, I_{A}^{j}-\Delta t I_{A}^{j *}, I_{D}^{j}-\Delta t I_{D}^{j *}, \\
I_{R}^{j}-\Delta t I_{R}^{j *}, I_{T}^{j}-\Delta t I_{T}^{j *}, R^{j}-\Delta t R^{*}, D^{j}-\Delta t D^{j *}, V^{j}-\Delta t V^{j *}
\end{array}\right) \\
+I^{*}\left(\begin{array}{c}
t_{j-2}, S^{j}-\Delta t S^{j *}-\Delta t S^{(j-1) *}, I^{j}-\Delta t I^{j *}-\Delta t I^{(j-1) *}, \\
I_{A}^{j}-\Delta t I_{A}^{j *}-\Delta t I_{A}^{(j-1) *}, I_{D}^{j}-\Delta t I_{D}^{j *}-\Delta t I_{D}^{(j-1) *}, \\
I_{R}^{j}-\Delta t I_{R}^{j *}-\Delta t I_{R}^{(j-1) *}, I_{T}^{j}-\Delta t I_{T}^{j *}-\Delta t I_{T}^{(j-1) *}, \\
R^{j}-\Delta t R^{j *}-\Delta t R^{(j-1) *}, D^{j}-\Delta t D^{j *}-\Delta t D^{(j-1) *}, \\
V^{j}-\Delta t V^{j *}-\Delta t V^{(j-1) *}
\end{array}\right)
\end{array}\right]
\end{aligned}
$$

$\times \Delta$,

$$
\begin{aligned}
I_{A}^{n+1}= & \frac{1-\alpha}{A B(\alpha)} I_{A}^{*}\left(\begin{array}{c}
t_{n+1}, S^{n}+\Delta t S^{n *}, I^{n}+\Delta t I^{n *}, I_{A}^{n}+\Delta t I_{A}^{n *}, \\
I_{D}^{n}+\Delta t I_{D}^{n *}, I_{R}^{n}+\Delta t I_{R}^{n *}, I_{T}^{n}+\Delta t I_{T}^{n *}, \\
R^{n}+\Delta t R^{n *}, D^{n}+\Delta t D^{n *}, V^{n}+\Delta t V^{n *}
\end{array}\right) \\
& +\frac{\alpha(\Delta t)^{\alpha}}{A B(\alpha) \Gamma(\alpha+1)}
\end{aligned}
$$




$$
\begin{aligned}
& \times \sum_{j=2}^{n} I_{A}^{*}\left(\begin{array}{c}
t_{j-2}, S^{j}-\Delta t S^{j *}-\Delta t S^{(j-1) *}, I^{j}-\Delta t I^{j *}-\Delta t I^{(j-1) *}, \\
I_{A}^{j}-\Delta t I_{A}^{j *}-\Delta t I_{A}^{(j-1) *}, I_{D}^{j}-\Delta t I_{D}^{j *}-\Delta t I_{D}^{(j-1) *}, \\
I_{R}^{j}-\Delta t I_{R}^{j *}-\Delta t I_{R}^{(j-1) *}, I_{T}^{j}-\Delta t I_{T}^{j *}-\Delta t I_{T}^{(j-1) *}, \\
R^{j}-\Delta t R^{j *}-\Delta t R^{(j-1) *}, D^{j}-\Delta t D^{j *}-\Delta t D^{(j-1) *}, \\
V^{j}-\Delta t V^{j *}-\Delta t V^{(j-1) *}
\end{array}\right) \times \Pi \\
& +\frac{\alpha(\Delta t)^{\alpha}}{A B(\alpha) \Gamma(\alpha+2)}
\end{aligned}
$$

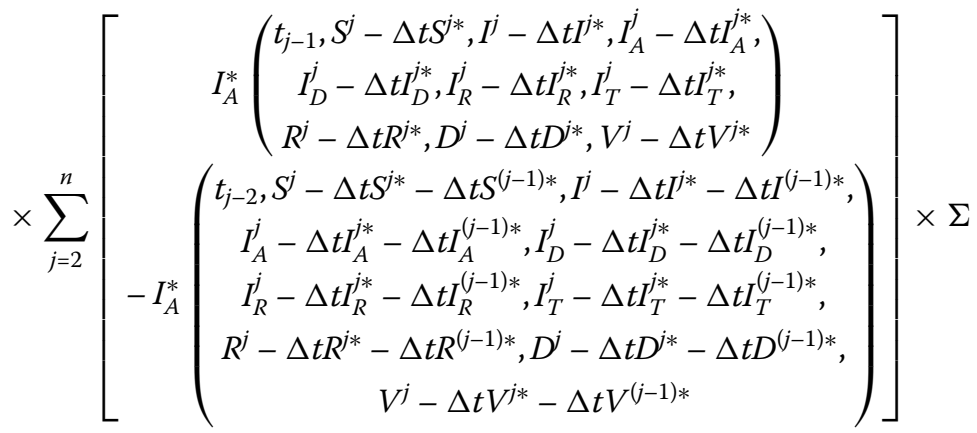

$$
\begin{aligned}
& +\frac{\alpha(\Delta t)^{\alpha}}{2 A B(\alpha) \Gamma(\alpha+3)} \\
& \times \sum_{j=2}^{n}\left[\begin{array}{c}
I_{A}^{*}\left(t_{j}, S^{j}, I^{j}, I_{A}^{j}, I_{D}^{j}, I_{R}^{j}, I_{T}^{j}, R^{j}, D^{j}, V^{j}\right) \\
t_{j-1}, S^{j}-\Delta t S^{j *}, I^{j}-\Delta t I^{j *}, I_{A}^{j}-\Delta t I_{A}^{j *}, \\
I_{D}^{j}-\Delta t I_{D}^{j *}, I_{R}^{j}-\Delta t I_{R}^{j *}, I_{T}^{j}-\Delta t I_{T}^{j *}, \\
R^{j}-\Delta t R^{j *}, D^{j}-\Delta t D^{j *}, V^{j}-\Delta t V^{j *}
\end{array}\right) \\
& I_{D}^{n+1}=\frac{1-\alpha}{A B(\alpha)} I_{D}^{*}\left(\begin{array}{c}
t_{n+1}, S^{n}+\Delta t S^{n *}, I^{n}+\Delta t I^{n *}, I_{A}^{n}+\Delta t I_{A}^{n *}, \\
I_{D}^{n}+\Delta t I_{D}^{n *}, I_{R}^{n}+\Delta t I_{R}^{n *}, I_{T}^{n}+\Delta t I_{T}^{n *}, \\
R^{n}+\Delta t R^{n *}, D^{n}+\Delta t D^{n *}, V^{n}+\Delta t V^{n *}
\end{array}\right) \\
& +\frac{\alpha(\Delta t)^{\alpha}}{A B(\alpha) \Gamma(\alpha+1)} \\
& \times \sum_{j=2}^{n} I_{D}^{*}\left(\begin{array}{c}
t_{j-2}, S^{j}-\Delta t S^{j *}-\Delta t S^{(j-1) *}, I^{j}-\Delta t I^{j *}-\Delta t I^{(j-1) *}, \\
I_{A}^{j}-\Delta t I_{A}^{j *}-\Delta t I_{A}^{(j-1) *}, I_{D}^{j}-\Delta t I_{D}^{j *}-\Delta t I_{D}^{(j-1) *}, \\
I_{R}^{j}-\Delta t I_{R}^{j *}-\Delta t I_{R}^{(j-1) *}, I_{T}^{j}-\Delta t I_{T}^{j *}-\Delta t I_{T}^{(j-1) *}, \\
R^{j}-\Delta t R^{j *}-\Delta t R^{(j-1) *}, D^{j}-\Delta t D^{j *}-\Delta t D^{(j-1) *}, \\
V^{j}-\Delta t V^{j *}-\Delta t V^{(j-1) *}
\end{array}\right) \times \Pi \\
& +\frac{\alpha(\Delta t)^{\alpha}}{A B(\alpha) \Gamma(\alpha+2)}
\end{aligned}
$$




$$
\begin{aligned}
& \left.\times \sum_{j=2}^{n}\left[\begin{array}{c}
t_{j-1}, S^{j}-\Delta t S^{j *}, I^{j}-\Delta t I^{j *}, I_{A}^{j}-\Delta t I_{A}^{j *}, \\
I_{D}^{j}-\Delta t I_{D}^{j *}, I_{R}^{j}-\Delta t I_{R}^{j *}, I_{T}^{j}-\Delta t I_{T}^{j *}, \\
R^{j}-\Delta t R^{j *}, D^{j}-\Delta t D^{j *}, V^{j}-\Delta t V^{j *}
\end{array}\right)\left(\begin{array}{c}
t_{j-2}, S^{j}-\Delta t S^{j *}-\Delta t S^{(j-1) *}, I^{j}-\Delta t I^{j *}-\Delta t I^{(j-1) *}, \\
I_{A}^{j}-\Delta t I_{A}^{j *}-\Delta t I_{A}^{(j-1) *}, I_{D}^{j}-\Delta t I_{D}^{j *}-\Delta t I_{D}^{(j-1) *}, \\
I_{R}^{j}-\Delta t I_{R}^{j *}-\Delta t I_{R}^{(j-1) *}, I_{T}^{j}-\Delta t I_{T}^{j *}-\Delta t I_{T}^{(j-1) *}, \\
R^{j}-\Delta t R^{j *}-\Delta t R^{(j-1) *}, D^{j}-\Delta t D^{j *}-\Delta t D^{(j-1) *}, \\
V^{j}-\Delta t V^{j *}-\Delta t V^{(j-1) *}
\end{array}\right)\right] \Sigma \\
& +\frac{\alpha(\Delta t)^{\alpha}}{2 A B(\alpha) \Gamma(\alpha+3)}
\end{aligned}
$$

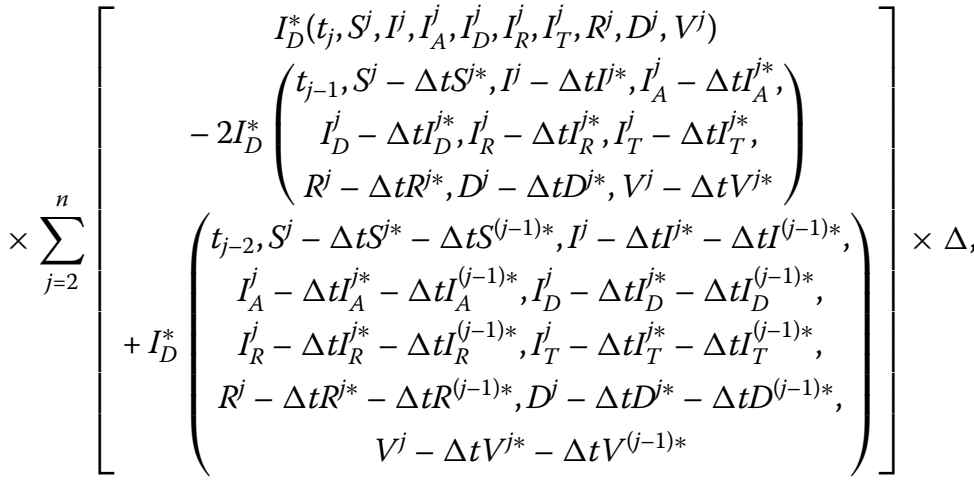

$$
\begin{aligned}
& I_{R}^{n+1}=\frac{1-\alpha}{A B(\alpha)} I_{R}^{*}\left(\begin{array}{c}
t_{n+1}, S^{n}+\Delta t S^{n *}, I^{n}+\Delta t I^{n *}, I_{A}^{n}+\Delta t I_{A}^{n *}, \\
I_{D}^{n}+\Delta t I_{D}^{n *}, I_{R}^{n}+\Delta t I_{R}^{n *}, I_{T}^{n}+\Delta t I_{T}^{n *}, \\
R^{n}+\Delta t R^{n *}, D^{n}+\Delta t D^{n *}, V^{n}+\Delta t V^{n *}
\end{array}\right) \\
& +\frac{\alpha(\Delta t)^{\alpha}}{A B(\alpha) \Gamma(\alpha+1)} \\
& \times \sum_{j=2}^{n} I_{R}^{*}\left(\begin{array}{c}
t_{j-2}, S^{j}-\Delta t S^{j *}-\Delta t S^{(j-1) *}, I^{j}-\Delta t I^{j *}-\Delta t I^{(j-1) *}, \\
I_{A}^{j}-\Delta t I_{A}^{j *}-\Delta t I_{A}^{(j-1) *}, I_{D}^{j}-\Delta t I_{D}^{j *}-\Delta t I_{D}^{(j-1) *}, \\
I_{R}^{j}-\Delta t I_{R}^{j *}-\Delta t I_{R}^{(j-1) *}, I_{T}^{j}-\Delta t I_{T}^{j *}-\Delta t I_{T}^{(j-1) *}, \\
R^{j}-\Delta t R^{j *}-\Delta t R^{(j-1) *}, D^{j}-\Delta t D^{j *}-\Delta t D^{(j-1) *}, \\
V^{j}-\Delta t V^{j *}-\Delta t V^{(j-1) *}
\end{array}\right) \times \Pi \\
& +\frac{\alpha(\Delta t)^{\alpha}}{A B(\alpha) \Gamma(\alpha+2)} \\
& \left.\times \sum_{j=2}^{n}\left[\begin{array}{c}
t_{R-1}^{*}, S^{j}-\Delta t S^{j *}, I^{j}-\Delta t I^{j *}, I_{A}^{j}-\Delta t I_{A}^{j *}, \\
I_{D}^{j}-\Delta t I_{D}^{j *}, I_{R}^{j}-\Delta t I_{R}^{j *}, I_{T}^{j}-\Delta t I_{T}^{j *}, \\
R^{j}-\Delta t R^{j *}, D^{j}-\Delta t D^{j *}, V^{j}-\Delta t V^{j *}
\end{array}\right)\left(\begin{array}{c}
t_{j-2}, S^{j}-\Delta t S^{j *}-\Delta t S^{(j-1) *}, I^{j}-\Delta t I^{j *}-\Delta t I^{(j-1) *}, \\
I_{A}^{j}-\Delta t I_{A}^{j *}-\Delta t I_{A}^{(j-1) *}, I_{D}^{j}-\Delta t I_{D}^{j *}-\Delta t I_{D}^{(j-1) *}, \\
I_{R}^{j}-\Delta t I_{R}^{j *}-\Delta t I_{R}^{(j-1) *}, I_{T}^{j}-\Delta t I_{T}^{j *}-\Delta t I_{T}^{(j-1) *}, \\
R^{j}-\Delta t R^{j *}-\Delta t R^{(j-1) *}, D^{j}-\Delta t D^{j *}-\Delta t D^{(j-1) *}, \\
V^{j}-\Delta t V^{j *}-\Delta t V^{(j-1) *}
\end{array}\right)\right] \Sigma \\
& +\frac{\alpha(\Delta t)^{\alpha}}{2 A B(\alpha) \Gamma(\alpha+3)}
\end{aligned}
$$




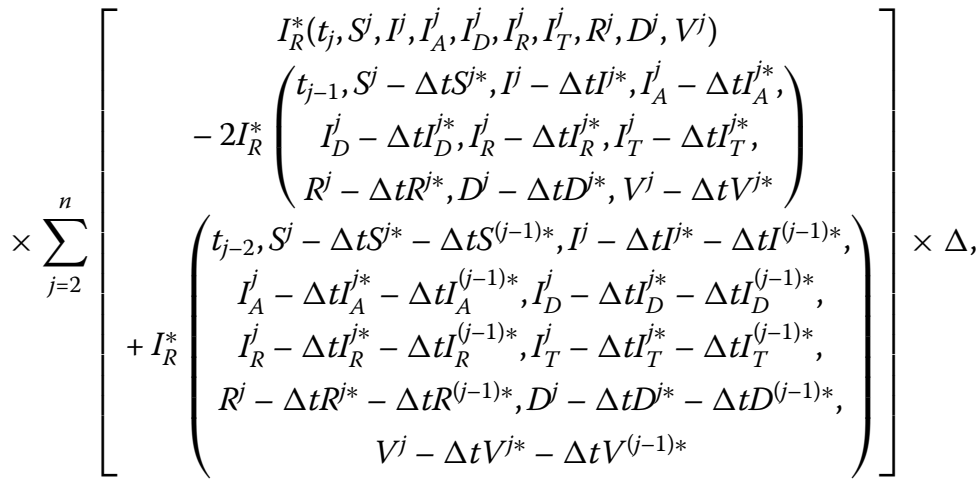

$$
\begin{aligned}
& I_{T}^{n+1}=\frac{1-\alpha}{A B(\alpha)} I_{T}^{*}\left(\begin{array}{c}
t_{n+1}, S^{n}+\Delta t S^{n *}, I^{n}+\Delta t I^{n *}, I_{A}^{n}+\Delta t I_{A}^{n *}, \\
I_{D}^{n}+\Delta t I_{D}^{n *}, I_{R}^{n}+\Delta t I_{R}^{n *}, I_{T}^{n}+\Delta t I_{T}^{n *}, \\
R^{n}+\Delta t R^{n *}, D^{n}+\Delta t D^{n *}, V^{n}+\Delta t V^{n *}
\end{array}\right) \\
& +\frac{\alpha(\Delta t)^{\alpha}}{A B(\alpha) \Gamma(\alpha+1)} \\
& \times \sum_{j=2}^{n} I_{T}^{*}\left(\begin{array}{c}
t_{j-2}, S^{j}-\Delta t S^{j *}-\Delta t S^{(j-1) *}, I^{j}-\Delta t I^{j *}-\Delta t I^{(j-1) *}, \\
I_{A}^{j}-\Delta t I_{A}^{j *}-\Delta t I_{A}^{(j-1) *}, I_{D}^{j}-\Delta t I_{D}^{j *}-\Delta t I_{D}^{(j-1) *}, \\
I_{R}^{j}-\Delta t I_{R}^{j *}-\Delta t I_{R}^{(j-1) *}, I_{T}^{j}-\Delta t I_{T}^{j *}-\Delta t I_{T}^{(j-1) *}, \\
R^{j}-\Delta t R^{j *}-\Delta t R^{(j-1) *}, D^{j}-\Delta t D^{j *}-\Delta t D^{(j-1) *}, \\
V^{j}-\Delta t V^{j *}-\Delta t V^{(j-1) *}
\end{array}\right) \times \Pi \\
& +\frac{\alpha(\Delta t)^{\alpha}}{A B(\alpha) \Gamma(\alpha+2)}
\end{aligned}
$$

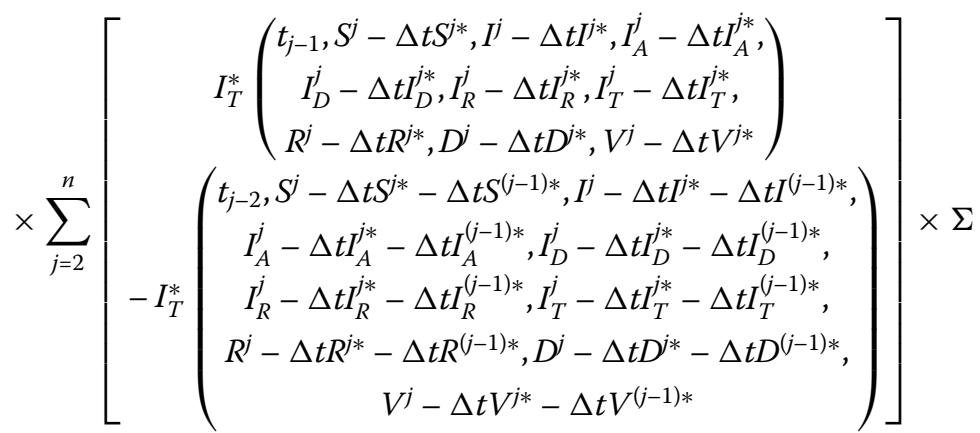

$$
\begin{aligned}
& +\frac{\alpha(\Delta t)^{\alpha}}{2 A B(\alpha) \Gamma(\alpha+3)}
\end{aligned}
$$

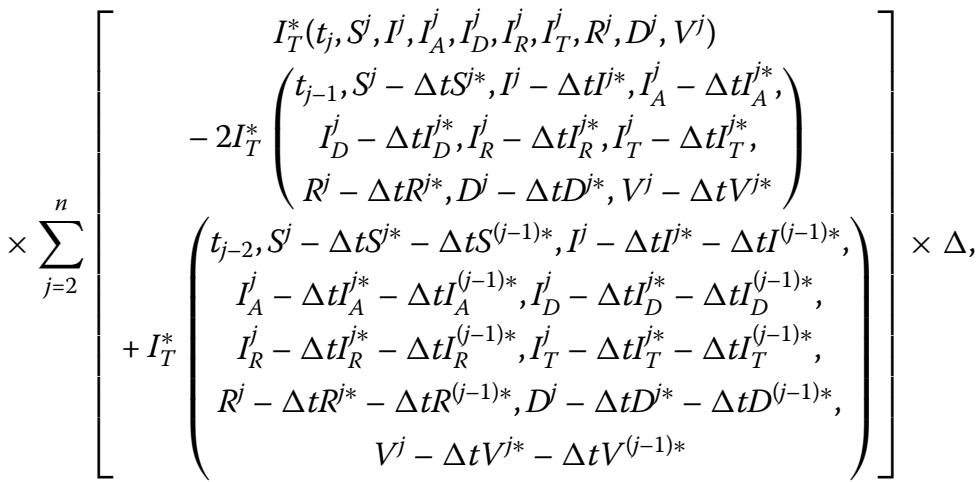




$$
\begin{aligned}
& R^{n+1}=\frac{1-\alpha}{A B(\alpha)} R^{*}\left(\begin{array}{c}
t_{n+1}, S^{n}+\Delta t S^{n *}, I^{n}+\Delta t I^{n *}, I_{A}^{n}+\Delta t I_{A}^{n *}, \\
I_{D}^{n}+\Delta t I_{D}^{n *}, I_{R}^{n}+\Delta t I_{R}^{n *}, I_{T}^{n}+\Delta t I_{T}^{n *}, \\
R^{n}+\Delta t R^{n *}, D^{n}+\Delta t D^{n *}, V^{n}+\Delta t V^{n *}
\end{array}\right) \\
& +\frac{\alpha(\Delta t)^{\alpha}}{A B(\alpha) \Gamma(\alpha+1)} \\
& \times \sum_{j=2}^{n} R^{*}\left(\begin{array}{c}
t_{j-2}, S^{j}-\Delta t S^{j *}-\Delta t S^{(j-1) *}, I^{j}-\Delta t I^{j *}-\Delta t I^{(j-1) *}, \\
I_{A}^{j}-\Delta t I_{A}^{j *}-\Delta t I_{A}^{(j-1) *}, I_{D}^{j}-\Delta t I_{D}^{j *}-\Delta t I_{D}^{(j-1) *}, \\
I_{R}^{j}-\Delta t I_{R}^{j *}-\Delta t I_{R}^{(j-1) *}, I_{T}^{j}-\Delta t I_{T}^{j *}-\Delta t I_{T}^{(j-1) *}, \\
R^{j}-\Delta t R^{j *}-\Delta t R^{(j-1) *}, D^{j}-\Delta t D^{j *}-\Delta t D^{(j-1) *}, \\
V^{j}-\Delta t V^{j *}-\Delta t V^{(j-1) *}
\end{array}\right) \times \Pi \\
& +\frac{\alpha(\Delta t)^{\alpha}}{A B(\alpha) \Gamma(\alpha+2)}
\end{aligned}
$$

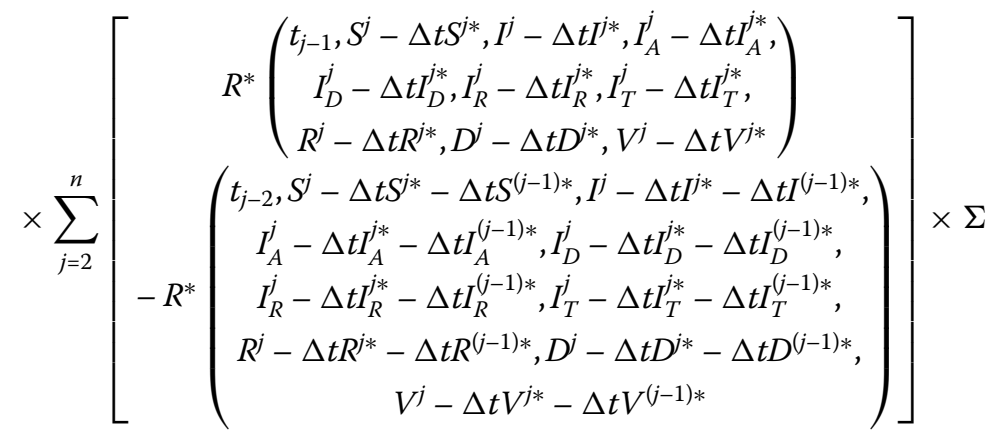

$$
\begin{aligned}
& +\frac{\alpha(\Delta t)^{\alpha}}{2 A B(\alpha) \Gamma(\alpha+3)} \\
& \left.\times \sum_{j=2}^{n}\left[\begin{array}{c}
R^{*}\left(t_{j}, S^{j}, I^{j}, I_{A}^{j}, I_{D}^{j}, I_{R}^{j}, I_{T}^{j}, R^{j}, D^{j}, V^{j}\right) \\
t_{j-1}, S^{j}-\Delta t S^{j *}, I^{j}-\Delta t I^{j *}, I_{A}^{j}-\Delta t I_{A}^{j *}, \\
I_{D}^{j}-\Delta t I_{D}^{j *}, I_{R}^{j}-\Delta t I_{R}^{*}, I_{T}^{j}-\Delta t I_{T}^{j *}, \\
R^{j}-\Delta t R^{j *}, D^{j}-\Delta t D^{j *}, V^{j}-\Delta t V^{j *}
\end{array}\right)+\left(\begin{array}{c}
t_{j-2}, S^{j}-\Delta t S^{j *}-\Delta t S^{(j-1) *}, I^{j}-\Delta t I^{j *}-\Delta t I^{(j-1) *}, \\
I_{A}^{j}-\Delta t I_{A}^{j *}-\Delta t I_{A}^{(j-1) *}, I_{D}^{j}-\Delta t I_{D}^{* *}-\Delta t I_{D}^{(j-1) *}, \\
I_{R}^{j}-\Delta t I_{R}^{j *}-\Delta t I_{R}^{(j-1) *}, I_{T}^{j}-\Delta t I_{T}^{j *}-\Delta t I_{T}^{(j-1) *}, \\
R^{j}-\Delta t R^{j *}-\Delta t R^{(j-1) *}, D^{j}-\Delta t D^{j *}-\Delta t D^{(j-1) *}, \\
V^{j}-\Delta t V^{j *}-\Delta t V^{(j-1) *}
\end{array}\right)\right] \times \Delta, \\
& D^{n+1}=\frac{1-\alpha}{A B(\alpha)} D^{*}\left(\begin{array}{c}
t_{n+1}, S^{n}+\Delta t S^{n *}, I^{n}+\Delta t I^{n *}, I_{A}^{n}+\Delta t I_{A}^{n *}, \\
I_{D}^{n}+\Delta t I_{D}^{n *}, I_{R}^{n}+\Delta t I_{R}^{n *}, I_{T}^{n}+\Delta t I_{T}^{n *}, \\
R^{n}+\Delta t R^{n *}, D^{n}+\Delta t D^{n *}, V^{n}+\Delta t V^{n *}
\end{array}\right) \\
& +\frac{\alpha(\Delta t)^{\alpha}}{A B(\alpha) \Gamma(\alpha+1)}
\end{aligned}
$$




$$
\begin{aligned}
& \times \sum_{j=2}^{n} D^{*}\left(\begin{array}{c}
t_{j-2}, S^{j}-\Delta t S^{j *}-\Delta t S^{(j-1) *}, I^{j}-\Delta t I^{j *}-\Delta t I^{(j-1) *}, \\
I_{A}^{j}-\Delta t I_{A}^{j *}-\Delta t I_{A}^{(j-1) *}, I_{D}^{j}-\Delta t I_{D}^{j *}-\Delta t I_{D}^{(j-1) *}, \\
I_{R}^{j}-\Delta t I_{R}^{j *}-\Delta t I_{R}^{(j-1) *}, I_{T}^{j}-\Delta t I_{T}^{j *}-\Delta t I_{T}^{(j-1) *}, \\
R^{j}-\Delta t R^{j *}-\Delta t R^{(j-1) *}, D^{j}-\Delta t D^{j *}-\Delta t D^{(j-1) *}, \\
V^{j}-\Delta t V^{j *}-\Delta t V^{(j-1) *}
\end{array}\right) \times \Pi \\
& +\frac{\alpha(\Delta t)^{\alpha}}{A B(\alpha) \Gamma(\alpha+2)}
\end{aligned}
$$

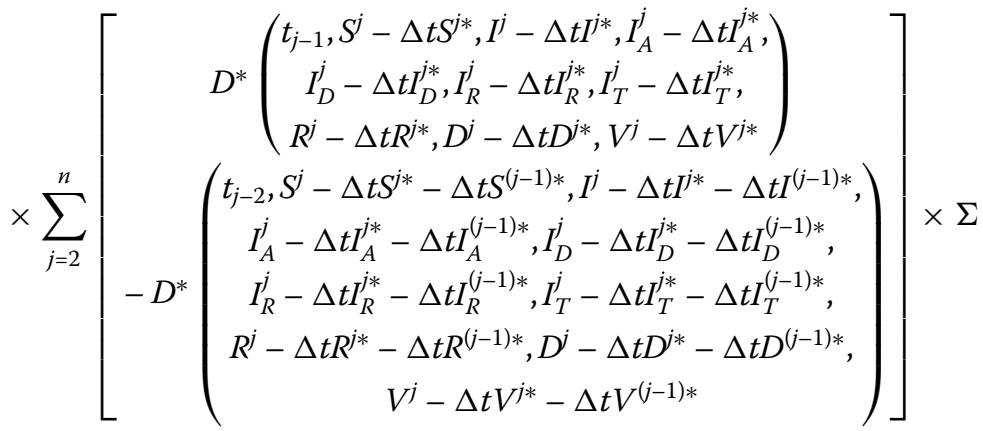

$$
\begin{aligned}
& +\frac{\alpha(\Delta t)^{\alpha}}{2 A B(\alpha) \Gamma(\alpha+3)} \\
& \times \sum_{j=2}^{n}\left[\begin{array}{c}
D^{*}\left(t_{j}, S^{j}, I^{j}, I_{A}^{j}, I_{D}^{j}, I_{R}^{j}, I_{T}^{j}, R^{j}, D^{j}, V^{j}\right) \\
t_{j-1}, S^{j}-\Delta t S^{j *}, I^{j}-\Delta t I^{j *}, I_{A}^{j}-\Delta t I_{A}^{j *}, \\
I_{D}^{j}-\Delta t I_{D}^{j *}, I_{R}^{j}-\Delta t I_{R}^{j *}, I_{T}^{j}-\Delta t I_{T}^{j *}, \\
R^{j}-\Delta t R^{j *}, D^{j}-\Delta t D^{j *}, V^{j}-\Delta t V^{j *}
\end{array}\right)+\left(\begin{array}{c}
t_{j-2}, S^{j}-\Delta t S^{j *}-\Delta t S^{(j-1) *}, I^{j}-\Delta t I^{j *}-\Delta t I^{(j-1) *}, \\
I_{A}^{j}-\Delta t I_{A}^{j *}-\Delta t I_{A}^{(j-1) *}, I_{D}^{j}-\Delta t I_{D}^{j *}-\Delta t I_{D}^{(j-1) *}, \\
I_{R}^{j}-\Delta t I_{R}^{j *}-\Delta t I_{R}^{(j-1) *}, I_{T}^{j}-\Delta t I_{T}^{j *}-\Delta t I_{T}^{(j-1) *}, \\
R^{j}-\Delta t R^{j *}-\Delta t R^{(j-1) *}, D^{j}-\Delta t D^{j *}-\Delta t D^{(j-1) *}, \\
V^{j}-\Delta t V^{j *}-\Delta t V^{(j-1) *}
\end{array}\right) \times \Delta, \\
& V^{n+1}=\frac{1-\alpha}{A B(\alpha)} V^{*}\left(\begin{array}{c}
t_{n+1}, S^{n}+\Delta t S^{n *}, I^{n}+\Delta t I^{n *}, I_{A}^{n}+\Delta t I_{A}^{n *}, \\
I_{D}^{n}+\Delta t I_{D}^{n *}, I_{R}^{n}+\Delta t I_{R}^{n *}, I_{T}^{n}+\Delta t I_{T}^{n *}, \\
R^{n}+\Delta t R^{n *}, D^{n}+\Delta t D^{n *}, V^{n}+\Delta t V^{n *}
\end{array}\right) \\
& +\frac{\alpha(\Delta t)^{\alpha}}{A B(\alpha) \Gamma(\alpha+1)} \\
& \times \sum_{j=2}^{n} V^{*}\left(\begin{array}{c}
t_{j-2}, S^{j}-\Delta t S^{j *}-\Delta t S^{(j-1) *}, I^{j}-\Delta t I^{j *}-\Delta t I^{(j-1) *}, \\
I_{A}^{j}-\Delta t I_{A}^{j *}-\Delta t I_{A}^{(j-1) *}, I_{D}^{j}-\Delta t I_{D}^{j *}-\Delta t I_{D}^{(j-1) *}, \\
I_{R}^{j}-\Delta t I_{R}^{j *}-\Delta t I_{R}^{(j-1) *}, I_{T}^{j}-\Delta t I_{T}^{j *}-\Delta t I_{T}^{(j-1) *}, \\
R^{j}-\Delta t R^{j *}-\Delta t R^{(j-1) *}, D^{j}-\Delta t D^{j *}-\Delta t D^{(j-1) *}, \\
V^{j}-\Delta t V^{j *}-\Delta t V^{(j-1) *}
\end{array}\right) \times \Pi \\
& +\frac{\alpha(\Delta t)^{\alpha}}{A B(\alpha) \Gamma(\alpha+2)}
\end{aligned}
$$




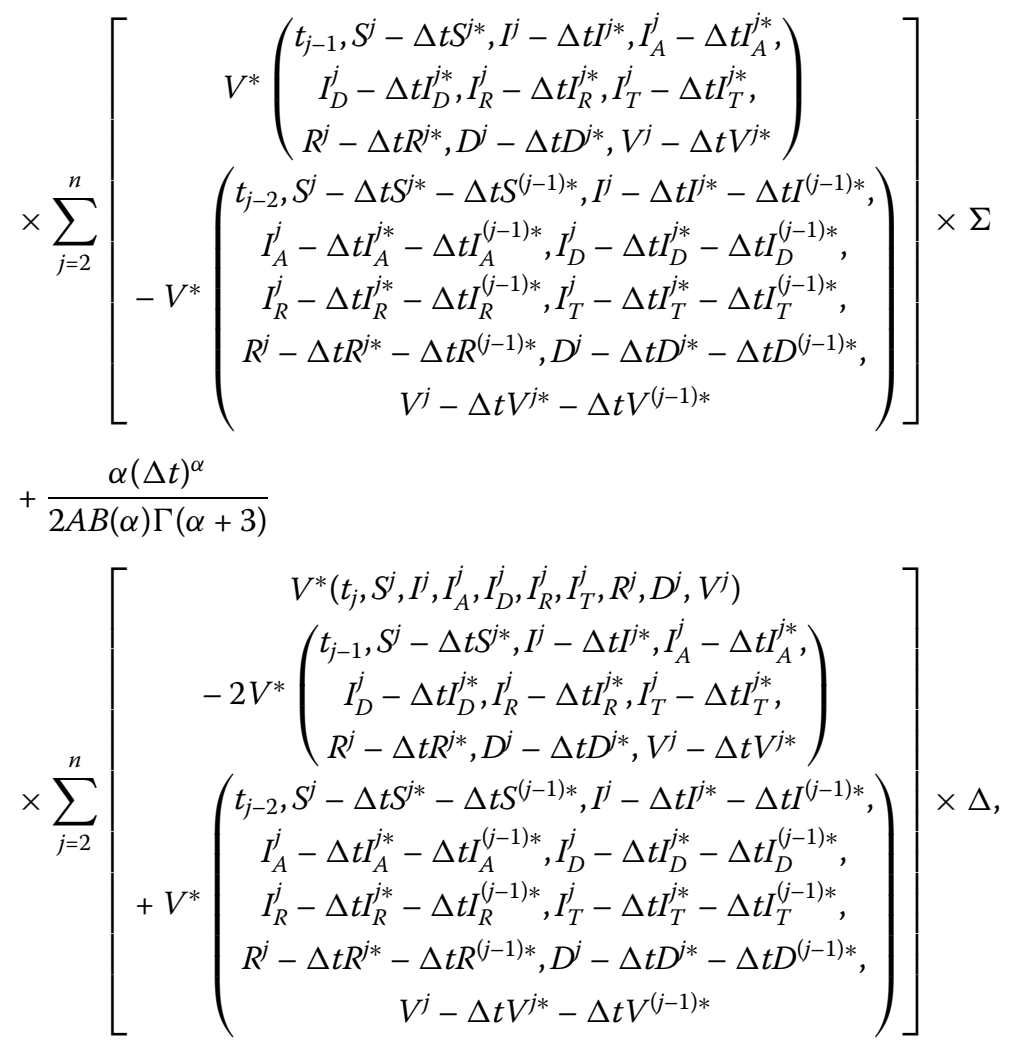

where

$$
\begin{aligned}
& \Delta=\left[\begin{array}{c}
(n-j+1)^{\alpha}\left[\begin{array}{c}
2(n-j)^{2}+(3 \alpha+10)(n-j) \\
+2 \alpha^{2}+9 \alpha+12
\end{array}\right] \\
-(n-j)^{\alpha}\left[\begin{array}{c}
2(n-j)^{2}+(5 \alpha+10)(n-j) \\
+6 \alpha^{2}+18 \alpha+12
\end{array}\right]
\end{array}\right], \\
& \Sigma=\left[\begin{array}{c}
(n-j+1)^{\alpha}(n-j+3+2 \alpha) \\
-(n-j)^{\alpha}(n-j+3+3 \alpha)
\end{array}\right], \quad \Pi=\left[(n-j+1)^{\alpha}-(n-j)^{\alpha}\right] .
\end{aligned}
$$


With the Caputo fractional derivative, we can obtain the following:

$$
\begin{aligned}
& S^{n+1}=\frac{(\Delta t)^{\alpha}}{\Gamma(\alpha+1)} \sum_{j=2}^{n} S^{*}\left(\begin{array}{c}
t_{j-2}, S^{j}-\Delta t S^{j *}-\Delta t S^{(j-1) *}, I^{j}-\Delta t I^{j *}-\Delta t I^{(j-1) *}, \\
I_{A}^{j}-\Delta t I_{A}^{j *}-\Delta t I_{A}^{(j-1) *}, I_{D}^{j}-\Delta t I_{D}^{j *}-\Delta t I_{D}^{(j-1) *}, \\
I_{R}^{j}-\Delta t I_{R}^{j *}-\Delta t I_{R}^{(j-1) *}, I_{T}^{j}-\Delta t I_{T}^{j *}-\Delta t I_{T}^{(j-1) *}, \\
R^{j}-\Delta t R^{j *}-\Delta t R^{(j-1) *}, D^{j}-\Delta t D^{j *}-\Delta t D^{(j-1) *}, \\
V^{j}-\Delta t V^{j *}-\Delta t V^{(j-1) *}
\end{array}\right) \times \Pi \\
& \left.+\frac{(\Delta t)^{\alpha}}{\Gamma(\alpha+2)} \sum_{j=2}^{n}\left[\begin{array}{c}
t_{j-1}, S^{j}-\Delta t S^{j *}, I^{j}-\Delta t I^{j *}, I_{A}^{j}-\Delta t I_{A}^{j *}, \\
I_{D}^{j}-\Delta t I_{D}^{j *}, I_{R}^{j}-\Delta t I_{R}^{j *}, I_{T}^{j}-\Delta t I_{T}^{j *}, \\
R^{j}-\Delta t R^{j *}, D^{j}-\Delta t D^{j *}, V^{j}-\Delta t V^{j *}
\end{array}\right)\left(\begin{array}{c}
t_{j-2}, S^{j}-\Delta t S^{j *}-\Delta t S^{(j-1) *}, I^{j}-\Delta t I^{j *}-\Delta t I^{(j-1) *}, \\
I_{A}^{j}-\Delta t I_{A}^{j *}-\Delta t I_{A}^{(j-1) *}, I_{D}^{j}-\Delta t I_{D}^{j *}-\Delta t I_{D}^{(j-1) *}, \\
I_{R}^{j}-\Delta t I_{R}^{j *}-\Delta t I_{R}^{(j-1) *}, I_{T}^{j}-\Delta t I_{T}^{j *}-\Delta t I_{T}^{(j-1) *}, \\
R^{j}-\Delta t R^{j *}-\Delta t R^{(j-1) *}, D^{j}-\Delta t D^{j *}-\Delta t D^{(j-1) *}, \\
V^{j}-\Delta t V^{j *}-\Delta t V^{(j-1) *}
\end{array}\right)\right] \\
& +\frac{(\Delta t)^{\alpha}}{2 \Gamma(\alpha+3)} \sum_{j=2}^{n}\left[\begin{array}{c}
S^{*}\left(t_{j}, S^{j}, I^{j}, I_{A}^{j}, I_{D}^{j}, I_{R}^{j}, I_{T}^{j}, R^{j}, D^{j}, V^{j}\right) \\
t_{j-1}, S^{j}-\Delta t S^{j *}, I^{j}-\Delta t I^{j *}, I_{A}^{j}-\Delta t I_{A}^{j *}, \\
I_{D}^{j}-\Delta t I_{D}^{j *}, I_{R}^{j}-\Delta t I_{R}^{j *}, I_{T}^{j}-\Delta t I_{T}^{j *}, \\
R^{j}-\Delta t R^{j *}, D^{j}-\Delta t D^{j *}, V^{j}-\Delta t V^{j *}
\end{array}\right)+S^{(j-1) *},\left(\begin{array}{c}
t_{j-2}, S^{j}-\Delta t S^{j *}-\Delta t S^{(j-1) *}, \Delta t I^{j *}-\Delta t I^{(j-1) *}, \\
I_{A}^{j}-\Delta t I_{A}^{j *}-\Delta t I_{A}^{(j-1) *}, I_{D}^{j}-\Delta t I_{D}^{j *}-\Delta t I_{D}^{(j-1) *}, \\
I_{R}^{j}-\Delta t I_{R}^{j *}-\Delta t I_{R}^{(j-1) *}, I_{T}^{j}-\Delta t I_{T}^{j *}-\Delta t I_{T}^{(j-1) *}, \\
R^{j}-\Delta t R^{j *}-\Delta t R^{(j-1) *}, D^{j}-\Delta t D^{j *}-\Delta t D^{(j-1) *}, \\
V^{j}-\Delta t V^{j *}-\Delta t V^{(j-1) *}
\end{array}\right)
\end{aligned}
$$

$\times \Delta$,

$$
\left.\begin{array}{l}
I^{n+1}=\frac{(\Delta t)^{\alpha}}{\Gamma(\alpha+1)} \sum_{j=2}^{n} I^{*}\left(\begin{array}{c}
t_{j-2}, S^{j}-\Delta t S^{j *}-\Delta t S^{(j-1) *}, I^{j}-\Delta t I^{j *}-\Delta t I^{(j-1) *}, \\
I_{A}^{j}-\Delta t I_{A}^{j *}-\Delta t I_{A}^{(j-1) *}, I_{D}^{j}-\Delta t I_{D}^{j *}-\Delta t I_{D}^{(j-1) *}, \\
I_{R}^{j}-\Delta t I_{R}^{j *}-\Delta t I_{R}^{(j-1) *}, I_{T}^{j}-\Delta t I_{T}^{j *}-\Delta t I_{T}^{(j-1) *}, \\
R^{j}-\Delta t R^{j *}-\Delta t R^{(j-1) *}, D^{j}-\Delta t D^{j *}-\Delta t D^{(j-1) *}, \\
V^{j}-\Delta t V^{j *}-\Delta t V^{(j-1) *}
\end{array}\right) \times \Pi \\
+\frac{(\Delta t)^{\alpha}}{\Gamma(\alpha+2)} \sum_{j=2}^{n}\left[\begin{array}{c}
t_{j-1}, S^{j}-\Delta t S^{j *}, I^{j}-\Delta t I^{j *}, I_{A}^{j}-\Delta t I_{A}^{j *}, \\
I_{D}^{j}-\Delta t I_{D}^{j *}, I_{R}^{j}-\Delta t I_{R}^{j *}, I_{T}^{j}-\Delta t I_{T}^{j *}, \\
R^{j}-\Delta t R^{j *}, D^{j}-\Delta t D^{j *}, V^{j}-\Delta t V^{j *}
\end{array}\right) \\
-I^{*}\left(\begin{array}{c}
t_{j-2}, S^{j}-\Delta t S^{j *}-\Delta t S^{(j-1) *}, I^{j}-\Delta t I^{j *}-\Delta t I^{(j-1) *}, \\
I_{A}^{j}-\Delta t I_{A}^{j *}-\Delta t I_{A}^{(j-1) *}, I_{D}^{j}-\Delta t I_{D}^{j *}-\Delta t I_{D}^{(j-1) *}, \\
I_{R}^{j}-\Delta t I_{R}^{j *}-\Delta t I_{R}^{(j-1) *}, I_{T}^{j}-\Delta t I_{T}^{j *}-\Delta t I_{T}^{(j-1) *}, \\
R^{j}-\Delta t R^{j *}-\Delta t R^{(j-1) *}, D^{j}-\Delta t D^{j *}-\Delta t D^{(j-1) *}, \\
V^{j}-\Delta t V^{j *}-\Delta t V^{(j-1) *}
\end{array}\right] \times \Sigma
\end{array}\right]
$$




$$
\left.+\frac{(\Delta t)^{\alpha}}{2 \Gamma(\alpha+3)} \sum_{j=2}^{n}\left[\begin{array}{c}
I^{*}\left(t_{j}, S^{j}, I^{j}, I_{A}^{j}, I_{D}^{j}, I_{R}^{j}, I_{T}^{j}, R^{j}, D^{j}, V^{j}\right) \\
t_{j-1}, S^{j}-\Delta t S^{j *}, I^{j}-\Delta t I^{j *}, I_{A}^{j}-\Delta t I_{A}^{j *}, \\
I_{D}^{j}-\Delta t I_{D}^{j *}, I_{R}^{j}-\Delta t I_{R}^{j *}, I_{T}^{j}-\Delta t I_{T}^{j *}, \\
R^{j}-\Delta t R^{j *}, D^{j}-\Delta t D^{j *}, V^{j}-\Delta t V^{j *}
\end{array}\right)+\left(\begin{array}{c}
t_{j-2}, S^{j}-\Delta t S^{j *}-\Delta t S^{(j-1) *}, I^{j}-\Delta t I^{j *}-\Delta t I^{(j-1) *}, \\
I_{A}^{j}-\Delta t I_{A}^{j *}-\Delta t I_{A}^{(j-1) *}, I_{D}^{j}-\Delta t I_{D}^{j *}-\Delta t I_{D}^{(j-1) *}, \\
I_{R}^{j}-\Delta t I_{R}^{j *}-\Delta t I_{R}^{(j-1) *}, I_{T}^{j}-\Delta t I_{T}^{j *}-\Delta t I_{T}^{(j-1) *}, \\
R^{j}-\Delta t R^{j *}-\Delta t R^{(j-1) *}, D^{j}-\Delta t D^{j *}-\Delta t D^{(j-1) *}, \\
V^{j}-\Delta t V^{j *}-\Delta t V^{(j-1) *}
\end{array}\right)\right]
$$

$\times \Delta$,

$$
\begin{aligned}
I_{A}^{n+1}=\frac{(\Delta t)^{\alpha}}{\Gamma(\alpha+1)} \sum_{j=2}^{n} I_{A}^{*}\left(\begin{array}{c}
t_{j-2}, S^{j}-\Delta t S^{j *}-\Delta t S^{(j-1) *}, I^{j}-\Delta t I^{j *}-\Delta t I^{(j-1) *}, \\
I_{A}^{j}-\Delta t I_{A}^{j *}-\Delta t I_{A}^{(j-1) *}, I_{D}^{j}-\Delta t I_{D}^{j *}-\Delta t I_{D}^{(j-1) *}, \\
I_{R}^{j}-\Delta t I_{R}^{j *}-\Delta t I_{R}^{(j-1) *}, I_{T}^{j}-\Delta t I_{T}^{j *}-\Delta t I_{T}^{(j-1) *}, \\
R^{j}-\Delta t R^{j *}-\Delta t R^{(j-1) *}, D^{j}-\Delta t D^{j *}-\Delta t D^{(j-1) *}, \\
V^{j}-\Delta t V^{j *}-\Delta t V^{(j-1) *}
\end{array}\right) \times \Pi \\
+\frac{(\Delta t)^{\alpha}}{\Gamma(\alpha+2)} \sum_{j=2}^{n}\left[\begin{array}{c}
I_{A}^{*}\left(\begin{array}{c}
t_{j-1}, S^{j}-\Delta t S^{j *}, I^{j}-\Delta t I^{j *}, I_{A}^{j}-\Delta t I_{A}^{j *} \\
I_{D}^{j}-\Delta t I_{D}^{j *}, I_{R}^{j}-\Delta t I_{R}^{* *}, I_{T}^{j}-\Delta t I_{T}^{j *}, \\
R^{j}-\Delta t R^{j *}, D^{j}-\Delta t D^{j *}, V^{j}-\Delta t V^{j *}
\end{array}\right) \\
-\left(\begin{array}{c}
t_{j-2}, S^{j}-\Delta t S^{j *}-\Delta t S^{(j-1) *}, I^{j}-\Delta t I^{j *}-\Delta t I^{(j-1) *}, \\
I_{A}^{j}-\Delta t I_{A}^{* *}-\Delta t I_{A}^{(j-1) *}, I_{D}^{j}-\Delta t I_{D}^{j *}-\Delta t I_{D}^{(j-1) *}, \\
I_{R}^{j}-\Delta t I_{R}^{j *}-\Delta t I_{R}^{(j-1) *}, I_{T}^{j}-\Delta t I_{T}^{*}-\Delta t I_{T}^{(j-1) *}, \\
R^{j}-\Delta t R^{j *}-\Delta t R^{(j-1) *}, D^{j}-\Delta t D^{j *}-\Delta t D^{(j-1) *}, \\
V^{j}-\Delta t V^{j *}-\Delta t V^{(j-1) *}
\end{array}\right)
\end{array}\right]
\end{aligned}
$$

$\times \Sigma$

$$
+\frac{(\Delta t)^{\alpha}}{2 \Gamma(\alpha+3)} \sum_{j=2}^{n}\left[\begin{array}{c}
I_{A}^{*}\left(t_{j}, S^{j}, I^{j}, I_{A}^{j}, I_{D}^{j}, I_{R}^{j}, I_{T}^{j}, R^{j}, D^{j}, V^{j}\right) \\
t_{j-1}, S^{j}-\Delta t S^{j *}, I^{j}-\Delta t I^{j *}, I_{A}^{j}-\Delta t I_{A}^{* *}, \\
I_{D}^{j}-\Delta t I_{D}^{j *}, I_{R}^{j}-\Delta t I_{R}^{j *}, I_{T}^{j}-\Delta t I_{T}^{j *}, \\
R^{j}-\Delta t R^{j *}, D^{j}-\Delta t D^{j *}, V^{j}-\Delta t V^{j *}
\end{array}\right)
$$

$\times \Delta$,

$$
I_{D}^{n+1}=\frac{(\Delta t)^{\alpha}}{\Gamma(\alpha+1)} \sum_{j=2}^{n} I_{D}^{*}\left(\begin{array}{c}
t_{j-2}, S^{j}-\Delta t S^{j *}-\Delta t S^{(j-1) *}, I^{j}-\Delta t I^{j *}-\Delta t I^{(j-1) *}, \\
I_{A}^{j}-\Delta t I_{A}^{j *}-\Delta t I_{A}^{(j-1) *}, I_{D}^{j}-\Delta t I_{D}^{j *}-\Delta t I_{D}^{(j-1) *}, \\
I_{R}^{j}-\Delta t I_{R}^{j *}-\Delta t I_{R}^{(j-1) *}, I_{T}^{j}-\Delta t I_{T}^{j *}-\Delta t I_{T}^{(j-1) *}, \\
R^{j}-\Delta t R^{j *}-\Delta t R^{(j-1) *}, D^{j}-\Delta t D^{j *}-\Delta t D^{(j-1) *}, \\
V^{j}-\Delta t V^{j *}-\Delta t V^{(j-1) *}
\end{array}\right) \times \Pi
$$


$+\frac{(\Delta t)^{\alpha}}{\Gamma(\alpha+2)}$

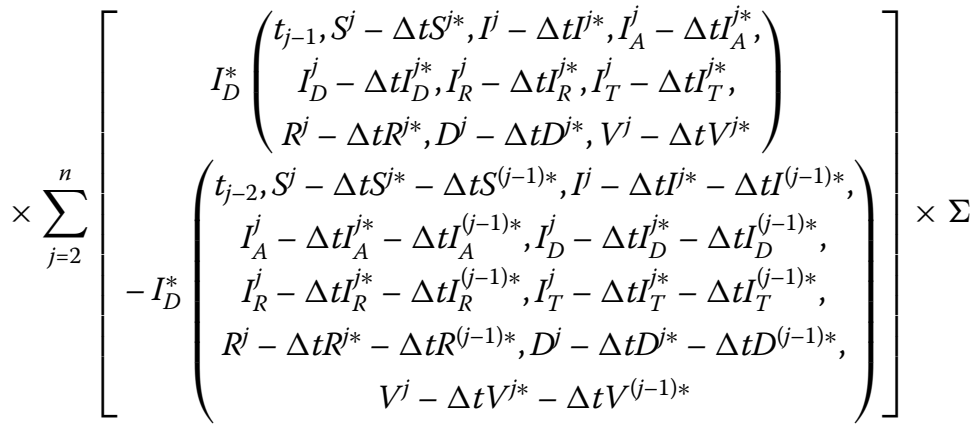

$+\frac{(\Delta t)^{\alpha}}{2 \Gamma(\alpha+3)}$

$\times \sum_{j=2}^{n}\left[\begin{array}{c}I_{D}^{*}\left(t_{j}, S^{j}, I^{j}, I_{A}^{j}, I_{D}^{j}, I_{R}^{j}, I_{T}^{j}, R^{j}, D^{j}, V^{j}\right) \\ -2 I_{D}^{*}\left(\begin{array}{c}t_{j-1}, S^{j}-\Delta t S^{j *}, I^{j}-\Delta t I^{j *}, I_{A}^{j}-\Delta t I_{A}^{j *}, I_{D}^{j}-\Delta t I_{D}^{j *}, \\ I_{R}^{j}-\Delta t I_{R}^{j *}, I_{T}^{j}-\Delta t I_{T}^{j *}, R^{j}-\Delta t R^{j *}, D^{j}-\Delta t D^{j *}, V^{j}-\Delta t V^{j *}\end{array}\right) \\ +I_{D}^{*}\left(\begin{array}{c}t_{j-2}, S^{j}-\Delta t S^{j *}-\Delta t S^{(j-1) *}, I^{j}-\Delta t I^{j *}-\Delta t I^{(j-1) *}, \\ I_{A}^{j}-\Delta t I_{A}^{j *}-\Delta t I_{A}^{(j-1) *}, I_{D}^{j}-\Delta t I_{D}^{j *}-\Delta t I_{D}^{(j-1) *}, \\ I_{R}^{j}-\Delta t I_{R}^{j *}-\Delta t I_{R}^{(j-1) *}, I_{T}^{j}-\Delta t I_{T}^{j}-\Delta t I_{T}^{(j-1) *}, \\ R^{j}-\Delta t R^{j *}-\Delta t R^{(j-1) *}, D^{j}-\Delta t D^{j *}-\Delta t D^{(j-1) *}, \\ V^{j}-\Delta t V^{j *}-\Delta t V^{(j-1) *}\end{array}\right)\end{array}\right]$

$\times \Delta$,

$$
\begin{aligned}
I_{R}^{n+1}=\frac{(\Delta t)^{\alpha}}{\Gamma(\alpha+1)} \sum_{j=2}^{n} I_{R}^{*}\left(\begin{array}{c}
t_{j-2}, S^{j}-\Delta t S^{j *}-\Delta t S^{(j-1) *}, I^{j}-\Delta t I^{j *}-\Delta t I^{(j-1) *}, \\
I_{A}^{j}-\Delta t I_{A}^{j *}-\Delta t I_{A}^{(j-1) *}, I_{D}^{j}-\Delta t I_{D}^{* *}-\Delta t I_{D}^{(j-1) *}, \\
I_{R}^{j}-\Delta t I_{R}^{j *}-\Delta t I_{R}^{(j-1) *}, I_{T}^{j}-\Delta t I_{T}^{j *}-\Delta t I_{T}^{(j-1) *}, \\
R^{j}-\Delta t R^{j *}-\Delta t R^{(j-1) *}, D^{j}-\Delta t D^{j *}-\Delta t D^{(j-1) *}, \\
V^{j}-\Delta t V^{j *}-\Delta t V^{(j-1) *}
\end{array}\right) \times \Pi \\
+\frac{(\Delta t)^{\alpha}}{\Gamma(\alpha+2)} \sum_{j=2}^{n}\left[\begin{array}{c}
I_{R}^{*}\left(\begin{array}{c}
t_{j-1}, S^{j}-\Delta t S^{j *}, I^{j}-\Delta t I^{j *}, I_{A}^{j}-\Delta t I_{A}^{j *}, \\
I_{D}^{j}-\Delta t I_{D}^{*}, I_{R}^{j}-\Delta t I_{R}^{j *}, I_{T}^{j}-\Delta t I_{T}^{j *}, \\
R^{j}-\Delta t R^{j *}, D^{j}-\Delta t D^{j *}, V^{j}-\Delta t V^{j *}
\end{array}\right) \\
-I_{R}^{*}\left(\begin{array}{c}
t_{j-2}, S^{j}-\Delta t S^{j *}-\Delta t S^{(j-1) *}, I^{j}-\Delta t I^{j *}-\Delta t I^{(j-1) *} \\
I_{A}^{j}-\Delta t I_{A}^{j *}-\Delta t I_{A}^{(j-1) *}, I_{D}^{j}-\Delta t I_{D}^{*}-\Delta t I_{D}^{(j-1) *}, \\
I_{R}^{j}-\Delta t I_{R}^{j *}-\Delta t I_{R}^{(j-1) *}, I_{T}^{j}-\Delta t I_{T}^{j *}-\Delta t I_{T}^{(j-1) *}, \\
R^{j}-\Delta t R^{j *}-\Delta t R^{(j-1) *}, D^{j}-\Delta t D^{j *}-\Delta t D^{(j-1) *}, \\
V^{j}-\Delta t V^{j *}-\Delta t V^{(j-1) *}
\end{array}\right)
\end{array}\right] \times \Sigma
\end{aligned}
$$




$$
\left.+\frac{(\Delta t)^{\alpha}}{2 \Gamma(\alpha+3)} \sum_{j=2}^{n}\left[\begin{array}{c}
I_{R}^{*}\left(t_{j}, S^{j}, I^{j}, I_{A}^{j}, I_{D}^{j}, I_{R}^{j}, I_{T}^{j}, R^{j}, D^{j}, V^{j}\right) \\
t_{j-1}, S^{j}-\Delta t S^{j *}, I^{j}-\Delta t I^{j *}, I_{A}^{j}-\Delta t I_{A}^{j *}, \\
I_{D}^{j}-\Delta t I_{D}^{j *}, I_{R}^{j}-\Delta t I_{R}^{j}, I_{T}^{j}-\Delta t I_{T}^{j *}, \\
R^{j}-\Delta t R^{j *}, D^{j}-\Delta t D^{j *}, V^{j}-\Delta t V^{j *}
\end{array}\right)+\left(\begin{array}{c}
t_{j-2}, S^{j}-\Delta t S^{j *}-\Delta t S^{(j-1) *}, I^{j}-\Delta t I^{j *}-\Delta t I^{(j-1) *}, \\
I_{A}^{j}-\Delta t I_{A}^{j *}-\Delta t I_{A}^{(j-1) *}, I_{D}^{j}-\Delta t I_{D}^{j *}-\Delta t I_{D}^{(j-1) *}, \\
I_{R}^{j}-\Delta t I_{R}^{j *}-\Delta t I_{R}^{(j-1) *}, I_{T}^{j}-\Delta t I_{T}^{j *}-\Delta t I_{T}^{(j-1) *}, \\
R^{j}-\Delta t R^{j *}-\Delta t R^{(j-1) *}, D^{j}-\Delta t D^{j *}-\Delta t D^{(j-1) *}, \\
V^{j}-\Delta t V^{j *}-\Delta t V^{(j-1) *}
\end{array}\right)\right]
$$

$\times \Delta$,

$$
\begin{aligned}
& I_{T}^{n+1}=\frac{(\Delta t)^{\alpha}}{\Gamma(\alpha+1)} \sum_{j=2}^{n} I_{T}^{*}\left(\begin{array}{c}
t_{j-2}, S^{j}-\Delta t S^{j *}-\Delta t S^{(j-1) *}, I^{j}-\Delta t I^{j *}-\Delta t I^{(j-1) *}, \\
I_{A}^{j}-\Delta t I_{A}^{j *}-\Delta t I_{A}^{(j-1) *}, I_{D}^{j}-\Delta t I_{D}^{j *}-\Delta t I_{D}^{(j-1) *}, \\
I_{R}^{j}-\Delta t I_{R}^{j *}-\Delta t I_{R}^{(j-1) *}, I_{T}^{j}-\Delta t I_{T}^{j *}-\Delta t I_{T}^{(j-1) *}, \\
R^{j}-\Delta t R^{j *}-\Delta t R^{(j-1) *}, D^{j}-\Delta t D^{j *}-\Delta t D^{(j-1) *}, \\
V^{j}-\Delta t V^{j *}-\Delta t V^{(j-1) *}
\end{array}\right) \times \Pi \\
& +\frac{\alpha(\Delta t)^{\alpha}}{\Gamma(\alpha+2)} \sum_{j=2}^{n}\left[\begin{array}{c}
t_{j-1}, S^{j}-\Delta t S^{j *}, I^{j}-\Delta t I^{j *}, I_{A}^{j}-\Delta t I_{A}^{j *}, \\
I_{D}^{j}-\Delta t I_{D}^{j *}, I_{R}^{j}-\Delta t I_{R}^{j *}, I_{T}^{j}-\Delta t I_{T}^{j *}, \\
R^{j}-\Delta t R^{j *}, D^{j}-\Delta t D^{j *}, V^{j}-\Delta t V^{j *}
\end{array}\right)\left(\begin{array}{c}
t_{j-2}, S^{j}-\Delta t S^{*}-\Delta t S^{(j-1) *}, I^{j}-\Delta t I^{j *}-\Delta t I^{(j-1) *}, \\
I_{A}^{j}-\Delta t I_{A}^{j *}-\Delta t I_{A}^{(j-1) *}, I_{D}^{j}-\Delta t I_{D}^{j *}-\Delta t I_{D}^{(j-1) *}, \\
I_{R}^{j}-\Delta t I_{R}^{j *}-\Delta t I_{R}^{(j-1) *}, I_{T}^{j}-\Delta t I_{T}^{j *}-\Delta t I_{T}^{(j-1) *}, \\
R^{j}-\Delta t R^{j *}-\Delta t R^{(j-1) *}, D^{j}-\Delta t D^{j *}-\Delta t D^{(j-1) *}, \\
V^{j}-\Delta t V^{j *}-\Delta t V^{(j-1) *}
\end{array}\right) \times \Sigma \\
& +\frac{(\Delta t)^{\alpha}}{2 \Gamma(\alpha+3)} \sum_{j=2}^{n}\left[\begin{array}{c}
I_{T}^{*}\left(t_{j}, S^{j}, I^{j}, I_{A}^{j}, I_{D}^{j}, I_{R}^{j}, I_{T}^{j}, R^{j}, D^{j}, V^{j}\right) \\
t_{j-1}, S^{j}-\Delta t S^{j *}, I^{j}-\Delta t I^{j *}, I_{A}^{j}-\Delta t I_{A}^{j *}, \\
I_{D}^{j}-\Delta t I_{D}^{j *}, I_{R}^{j}-\Delta t I_{R}^{j *}, I_{T}^{j}-\Delta t I_{T}^{j *}, \\
R^{j}-\Delta t R^{j *}, D^{j}-\Delta t D^{j *}, V^{j}-\Delta t V^{j *}
\end{array}\right)
\end{aligned}
$$

$\times \Delta$,

$$
R^{n+1}=\frac{(\Delta t)^{\alpha}}{\Gamma(\alpha+1)} \sum_{j=2}^{n} R^{*}\left(\begin{array}{c}
t_{j-2}, S^{j}-\Delta t S^{j *}-\Delta t S^{(j-1) *}, I^{j}-\Delta t I^{j *}-\Delta t I^{(j-1) *}, \\
I_{A}^{j}-\Delta t I_{A}^{j *}-\Delta t I_{A}^{(j-1) *}, I_{D}^{j}-\Delta t I_{D}^{j *}-\Delta t I_{D}^{(j-1) *}, \\
I_{R}^{j}-\Delta t I_{R}^{j *}-\Delta t I_{R}^{(j-1) *}, I_{T}^{j}-\Delta t I_{T}^{j *}-\Delta t I_{T}^{(j-1) *}, \\
R^{j}-\Delta t R^{j *}-\Delta t R^{(j-1) *}, D^{j}-\Delta t D^{j *}-\Delta t D^{(j-1) *}, \\
V^{j}-\Delta t V^{j *}-\Delta t V^{(j-1) *}
\end{array}\right) \times \Pi
$$


$\left.+\frac{(\Delta t)^{\alpha}}{\Gamma(\alpha+2)} \sum_{j=2}^{n}\left[\begin{array}{c}t_{j-1}, S^{j}-\Delta t S^{j *}, I^{j}-\Delta t I^{j *}, I_{A}^{j}-\Delta t I_{A}^{j *}, \\ I_{D}^{j}-\Delta t I_{D}^{j *}, I_{R}^{j}-\Delta t I_{R}^{j *}, I_{T}^{j}-\Delta t I_{T}^{j *}, \\ R^{j}-\Delta t R^{j *}, D^{j}-\Delta t D^{j *}, V^{j}-\Delta t V^{j *}\end{array}\right)\left(\begin{array}{c}t_{j-2}, S^{j}-\Delta t S^{j *}-\Delta t S^{(j-1) *}, I^{j}-\Delta t j^{j *}-\Delta t I^{(j-1) *} \\ I_{A}^{j}-\Delta t I_{A}^{j *}-\Delta t I_{A}^{(j-1) *}, I_{D}^{j}-\Delta t I_{D}^{j}-\Delta t I_{D}^{(j-1) *}, \\ I_{R}^{j}-\Delta t I_{R}^{j *}-\Delta t I_{R}^{(j-1) *}, I_{T}^{j}-\Delta t I_{T}^{j *}-\Delta t I_{T}^{(j-1) *}, \\ R^{j}-\Delta t R^{j *}-\Delta t R^{(j-1) *}, D^{j}-\Delta t D^{j *}-\Delta t D^{(j-1) *}, \\ V^{j}-\Delta t V^{j *}-\Delta t V^{(j-1) *}\end{array}\right)\right]$

$\times \Sigma$

$$
\left.+\frac{(\Delta t)^{\alpha}}{2 \Gamma(\alpha+3)} \sum_{j=2}^{n}\left[\begin{array}{c}
R^{*}\left(t_{j}, S^{j}, I^{j}, I_{A}^{j}, I_{D}^{j}, I_{R}^{j}, I_{T}^{j}, R^{j}, D^{j}, V^{j}\right) \\
t_{j-1}, S^{j}-\Delta t S^{j *}, I^{j}-\Delta t I^{j *}, I_{A}^{j}-\Delta t I_{A}^{j *}, \\
I_{D}^{j}-\Delta t I_{D}^{j *}, I_{R}^{j}-\Delta t I_{R}^{j *}, I_{T}^{j}-\Delta t I_{T}^{j *}, \\
R^{j}-\Delta t R^{j *}, D^{j}-\Delta t D^{j *}, V^{j}-\Delta t V^{j *}
\end{array}\right)+\left(\begin{array}{c}
t_{j-2}, S^{j}-\Delta t S^{j *}-\Delta t S^{(j-1) *}, I^{j}-\Delta t I^{j *}-\Delta t I^{(j-1) *}, \\
I_{A}^{j}-\Delta t I_{A}^{j *}-\Delta t I_{A}^{(j-1) *}, I_{D}^{j}-\Delta t I_{D}^{j *}-\Delta t I_{D}^{(j-1) *}, \\
I_{R}^{j}-\Delta t I_{R}^{j *}-\Delta t I_{R}^{(j-1) *}, I_{T}^{j}-\Delta t I_{T}^{j *}-\Delta t I_{T}^{(j-1) *}, \\
R^{j}-\Delta t R^{*}-\Delta t R^{(j-1) *}, D^{j}-\Delta t D^{j *}-\Delta t D^{(j-1) *}, \\
V^{j}-\Delta t V^{j *}-\Delta t V^{(j-1) *}
\end{array}\right)\right]
$$

$\times \Delta$,

$$
\begin{aligned}
& D^{n+1}=\frac{(\Delta t)^{\alpha}}{\Gamma(\alpha+1)} \sum_{j=2}^{n} D^{*}\left(\begin{array}{c}
t_{j-2}, S^{j}-\Delta t S^{j *}-\Delta t S^{(j-1) *}, I^{j}-\Delta t I^{j *}-\Delta t I^{(j-1) *}, \\
I_{A}^{j}-\Delta t I_{A}^{j *}-\Delta t I_{A}^{(j-1) *}, I_{D}^{j}-\Delta t I_{D}^{j *}-\Delta t I_{D}^{(j-1) *}, \\
I_{R}^{j}-\Delta t I_{R}^{j *}-\Delta t I_{R}^{(j-1) *}, I_{T}^{j}-\Delta t I_{T}^{j *}-\Delta t I_{T}^{(j-1) *}, \\
R^{j}-\Delta t R^{j *}-\Delta t R^{(j-1) *}, D^{j}-\Delta t D^{j *}-\Delta t D^{(j-1) *}, \\
V^{j}-\Delta t V^{j *}-\Delta t V^{(j-1) *}
\end{array}\right) \times \Pi
\end{aligned}
$$

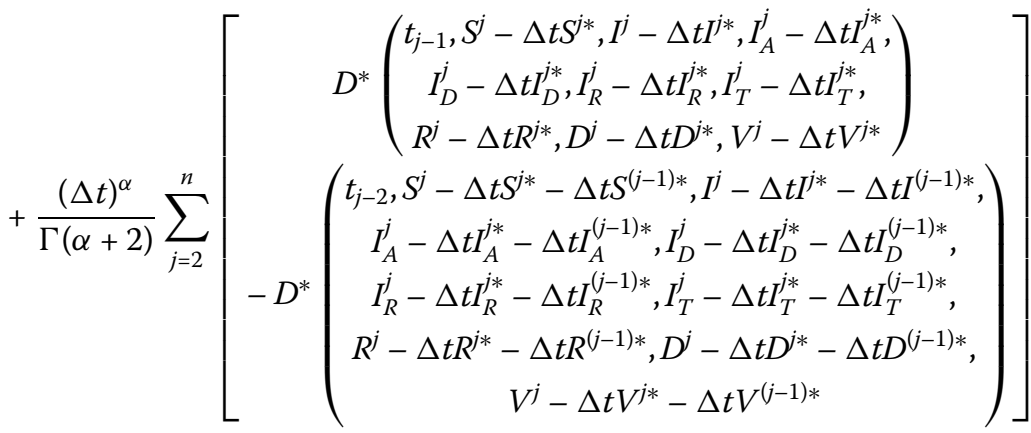

$\times \Sigma$ 


$$
\left.+\frac{(\Delta t)^{\alpha}}{2 \Gamma(\alpha+3)} \sum_{j=2}^{n}\left[\begin{array}{c}
D^{*}\left(t_{j}, S^{j}, I^{j}, I_{A}^{j}, I_{D}^{j}, I_{R}^{j}, I_{T}^{j}, R^{j}, D^{j}, V^{j}\right) \\
t_{j-1}, S^{j}-\Delta t S^{j *}, I^{j}-\Delta t I^{j *}, I_{A}^{j}-\Delta t I_{A}^{j *}, \\
I_{D}^{j}-\Delta t I_{D}^{j *}, I_{R}^{j}-\Delta t I_{R}^{j *}, I_{T}^{j}-\Delta t I_{T}^{j *}, \\
R^{j}-\Delta t R^{j *}, D^{j}-\Delta t D^{j *}, V^{j}-\Delta t V^{j *}
\end{array}\right)+\left(\begin{array}{c}
t_{j-2}, S^{j}-\Delta t S^{j *}-\Delta t S^{(j-1) *}, I^{j}-\Delta t I^{j *}-\Delta t I^{(j-1) *}, \\
I_{A}^{j}-\Delta t I_{A}^{j *}-\Delta t I_{A}^{(j-1) *}, I_{D}^{j}-\Delta t I_{D}^{j *}-\Delta t I_{D}^{(j-1) *}, \\
I_{R}^{j}-\Delta t I_{R}^{j *}-\Delta t I_{R}^{(j-1) *}, I_{T}^{j}-\Delta t I_{T}^{j *}-\Delta t I_{T}^{(j-1) *}, \\
R^{j}-\Delta t R^{*}-\Delta t R^{(j-1) *}, D^{j}-\Delta t D^{j *}-\Delta t D^{(j-1) *}, \\
V^{j}-\Delta t V^{j *}-\Delta t V^{(j-1) *}
\end{array}\right)\right]
$$

$\times \Delta$

$$
\begin{aligned}
& V^{n+1}=\frac{(\Delta t)^{\alpha}}{\Gamma(\alpha+1)} \sum_{j=2}^{n} V^{*}\left(\begin{array}{c}
t_{j-2}, S^{j}-\Delta t S^{j *}-\Delta t S^{(j-1) *}, I^{j}-\Delta t I^{j *}-\Delta t I^{(j-1) *}, \\
I_{A}^{j}-\Delta t I_{A}^{j *}-\Delta t I_{A}^{(j-1) *}, I_{D}^{j}-\Delta t I_{D}^{j *}-\Delta t I_{D}^{(j-1) *}, \\
I_{R}^{j}-\Delta t I_{R}^{j *}-\Delta t I_{R}^{(j-1) *}, I_{T}^{j}-\Delta t I_{T}^{j *}-\Delta t I_{T}^{(j-1) *}, \\
R^{j}-\Delta t R^{j *}-\Delta t R^{(j-1) *}, D^{j}-\Delta t D^{j *}-\Delta t D^{(j-1) *}, \\
V^{j}-\Delta t V^{j *}-\Delta t V^{(j-1) *}
\end{array}\right) \times \Pi \\
& +\frac{(\Delta t)^{\alpha}}{\Gamma(\alpha+2)} \sum_{j=2}^{n}\left[\begin{array}{c}
t_{j-1}, S^{j}-\Delta t S^{j *}, I^{j}-\Delta t I^{j *}, I_{A}^{j}-\Delta t I_{A}^{j *}, \\
I_{D}^{j}-\Delta t I_{D}^{j *}, I_{R}^{j}-\Delta t I_{R}^{j *}, I_{T}^{j}-\Delta t I_{T}^{j *}, \\
R^{j}-\Delta t R^{j *}, D^{j}-\Delta t D^{j *}, V^{j}-\Delta t V^{j *}
\end{array}\right)
\end{aligned}
$$

$\times \Sigma$

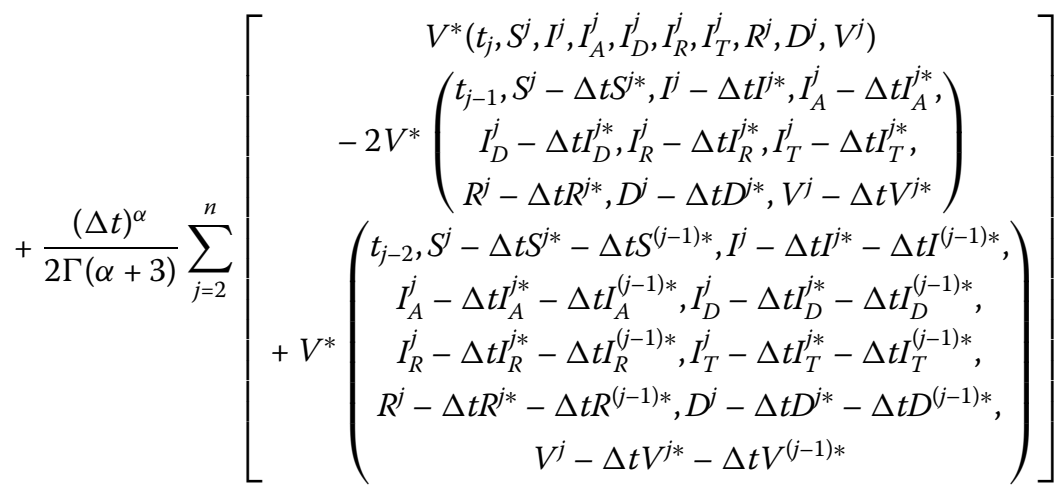

$\times \Delta$.

We now do the same routine for fractal-fractional derivatives. We start with the CaputoFabrizio fractal-fractional derivative

$$
\begin{aligned}
& { }_{0}^{F F E} D_{t}^{\alpha} S=S^{*}\left(t, S, I, I_{A}, I_{D}, I_{R}, I_{T}, R, D, V\right), \\
& { }_{0}^{F F E} D_{t}^{\alpha} I=I^{*}\left(t, S, I, I_{A}, I_{D}, I_{R}, I_{T}, R, D, V\right),
\end{aligned}
$$




$$
\begin{aligned}
& { }_{0}^{F F E} D_{t}^{\alpha} I_{A}=I_{A}^{*}\left(t, S, I, I_{A}, I_{D}, I_{R}, I_{T}, R, D, V\right), \\
& { }_{0}^{F F E} D_{t}^{\alpha} I_{D}=I_{D}^{*}\left(t, S, I, I_{A}, I_{D}, I_{R}, I_{T}, R, D, V\right), \\
& { }_{0}^{F F E} D_{t}^{\alpha} I_{R}=I_{R}^{*}\left(t, S, I, I_{A}, I_{D}, I_{R}, I_{T}, R, D, V\right), \\
& { }_{0}^{F F E} D_{t}^{\alpha} I_{T}=I_{T}^{*}\left(t, S, I, I_{A}, I_{D}, I_{R}, I_{T}, R, D, V\right), \\
& { }_{0}^{F F E} D_{t}^{\alpha} R=R^{*}\left(t, S, I, I_{A}, I_{D}, I_{R}, I_{T}, R, D, V\right), \\
& { }_{0}^{F F E} D_{t}^{\alpha} D=D^{*}\left(t, S, I, I_{A}, I_{D}, I_{R}, I_{T}, R, D, V\right), \\
& { }_{0}^{F F E} D_{t}^{\alpha} V=V^{*}\left(t, S, I, I_{A}, I_{D}, I_{R}, I_{T}, R, D, V\right) .
\end{aligned}
$$

After applying the fractional integral with exponential kernel and putting the Newton polynomial into these equations, we can solve our model as follows:

$$
\begin{aligned}
& S^{n+1}=S^{n}+\frac{1-\alpha}{M(\alpha)}\left[\begin{array}{c}
t_{n+1}^{1-\beta} S^{*}\left(\begin{array}{c}
t_{n+1}, S^{n}+\Delta t S^{n *}, I^{n}+\Delta t I^{n *}, I_{A}^{n}+\Delta t I_{A}^{n *}, \\
I_{D}^{n}+\Delta t I_{D}^{n *}, I_{R}^{n}+\Delta t I_{R}^{n *}, I_{T}^{n}+\Delta t I_{T}^{n *}, \\
R^{n}+\Delta t R^{n *}, D^{n}+\Delta t D^{n *}, V^{n}+\Delta t V^{n *}
\end{array}\right) \\
-t_{n}^{1-\beta} S^{*}\left(t_{n}, S^{n}, I^{n}, I_{A}^{n}, I_{D}^{n}, I_{R}^{n}, I_{T}^{n}, R^{n}, D^{n}, V^{n}\right)
\end{array}\right] \\
& +\frac{\alpha}{M(\alpha)}
\end{aligned}
$$

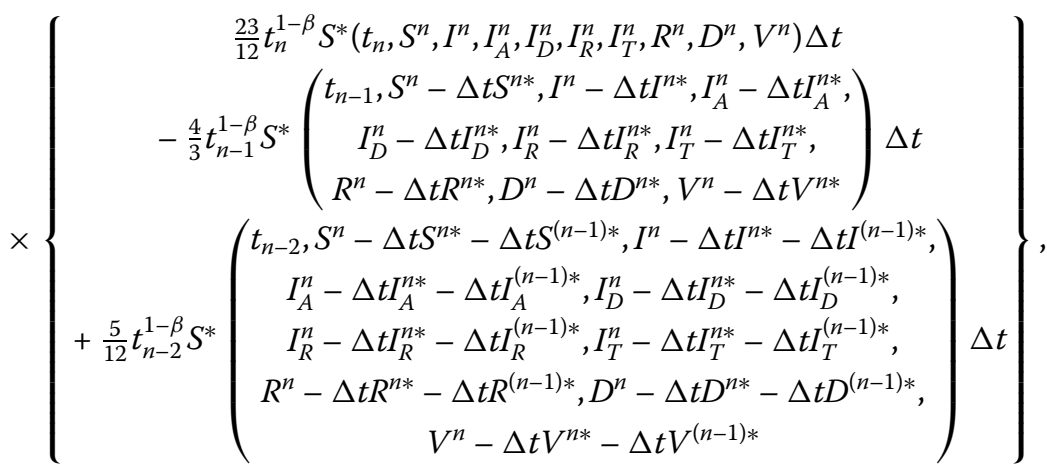

$$
\begin{aligned}
& I^{n+1}=S^{n}+\frac{1-\alpha}{M(\alpha)} \\
& {\left[\begin{array}{c}
t_{n+1}^{1-\beta} I^{*}\left(\begin{array}{c}
t_{n+1}, S^{n}+\Delta t S^{n *}, I^{n}+\Delta t I^{n *}, I_{A}^{n}+\Delta t I_{A}^{n *}, I_{D}^{n}+\Delta t I_{D}^{n *}, \\
I_{R}^{n}+\Delta t I_{R}^{n *}, I_{T}^{n}+\Delta t I_{T}^{n *}, R^{n}+\Delta t R^{n *}, D^{n}+\Delta t D^{n *}, V^{n}+\Delta t V^{n *}
\end{array}\right) \\
-t_{n}^{1-\beta} I^{*}\left(t_{n}, S^{n}, I^{n}, I_{A}^{n}, I_{D}^{n}, I_{R}^{n}, I_{T}^{n}, R^{n}, D^{n}, V^{n}\right)
\end{array}\right]} \\
& +\frac{\alpha}{M(\alpha)} \\
& \times\left\{\begin{array}{c}
\frac{23}{12} t_{n}^{1-\beta} I^{*}\left(t_{n}, S^{n}, I^{n}, I_{A}^{n}, I_{D}^{n}, I_{R}^{n}, I_{T}^{n}, R^{n}, D^{n}, V^{n}\right) \Delta t \\
-\frac{4}{3} t_{n-1}^{1-\beta} I^{*}\left(\begin{array}{c}
t_{n-1}, S^{n}-\Delta t S^{n *}, I^{n}-\Delta t I^{n *}, I_{A}^{n}-\Delta t I_{A}^{n *}, \\
I_{D}^{n}-\Delta t I_{D}^{n *}, I_{R}^{n}-\Delta t I_{R}^{n *}, I_{T}^{n}-\Delta t I_{T}^{n *}, \\
R^{n}-\Delta t R^{n *}, D^{n}-\Delta t D^{n *}, V^{n}-\Delta t V^{n *}
\end{array}\right) \Delta t \\
+\frac{5}{12} t_{n-2}^{1-\beta} I^{*}\left(\begin{array}{c}
t_{n-2}, S^{n}-\Delta t S^{n *}-\Delta t S^{(n-1) *}, I^{n}-\Delta t I^{n *}-\Delta t I^{(n-1) *}, \\
I_{A}^{n}-\Delta t I_{A}^{n *}-\Delta t I_{A}^{(n-1) *}, I_{D}^{n}-\Delta t I_{D}^{n *}-\Delta t I_{D}^{(n-1) *}, \\
I_{R}^{n}-\Delta t I_{R}^{n *}-\Delta t I_{R}^{(n-1) *}, I_{T}^{n}-\Delta t I_{T}^{n *}-\Delta t I_{T}^{(n-1) *}, \\
R^{n}-\Delta t R^{n *}-\Delta t R^{(n-1) *}, D^{n}-\Delta t D^{n *}-\Delta t D^{(n-1) *}, \\
V^{n}-\Delta t V^{n *}-\Delta t V^{(n-1) *}
\end{array}\right) \Delta t
\end{array}\right\},
\end{aligned}
$$




$$
\begin{aligned}
& I_{A}^{n+1}=I_{A}^{n}+\frac{1-\alpha}{M(\alpha)}\left[\begin{array}{c}
t_{n+1}^{1-\beta} I_{A}^{*}\left(\begin{array}{c}
t_{n+1}, S^{n}+\Delta t S^{n *}, I^{n}+\Delta t I^{n *}, I_{A}^{n}+\Delta t I_{A}^{n *}, \\
I_{D}^{n}+\Delta t I_{D}^{n *}, I_{R}^{n}+\Delta t I_{R}^{n *}, I_{T}^{n}+\Delta t I_{T}^{n *}, \\
R^{n}+\Delta t R^{n *}, D^{n}+\Delta t D^{n *}, V^{n}+\Delta t V^{n *}
\end{array}\right) \\
-t_{n}^{1-\beta} I_{A}^{*}\left(t_{n}, S^{n}, I^{n}, I_{A}^{n}, I_{D}^{n}, I_{R}^{n}, I_{T}^{n}, R^{n}, D^{n}, V^{n}\right)
\end{array}\right] \\
& +\frac{\alpha}{M(\alpha)} \\
& \times\left\{\begin{array}{c}
\frac{23}{12} t_{n}^{1-\beta} I_{A}^{*}\left(t_{n}, S^{n}, I^{n}, I_{A}^{n}, I_{D}^{n}, I_{R}^{n}, I_{T}^{n}, R^{n}, D^{n}, V^{n}\right) \Delta t \\
-\frac{4}{3} t_{n-1}^{1-\beta} I_{A}^{*}\left(\begin{array}{c}
t_{n-1}, S^{n}-\Delta t S^{n *}, I^{n}-\Delta t I^{n *}, I_{A}^{n}-\Delta t I_{A}^{n *}, \\
I_{D}^{n}-\Delta t I_{D}^{n *}, I_{R}^{n}-\Delta t I_{R}^{n *}, I_{T}^{n}-\Delta t I_{T}^{n *}, \\
R^{n}-\Delta t R^{n *}, D^{n}-\Delta t D^{n *}, V^{n}-\Delta t V^{n *}
\end{array}\right) \Delta t \\
+\frac{5}{12} t_{n-2}^{1-\beta} I_{A}^{*}\left(\begin{array}{c}
t_{n-2}, S^{n}-\Delta t S^{n *}-\Delta t S^{(n-1) *}, I^{n}-\Delta t I^{n *}-\Delta t I^{(n-1) *}, \\
I_{A}^{n}-\Delta t I_{A}^{n *}-\Delta t I_{A}^{(n-1) *}, I_{D}^{n}-\Delta t I_{D}^{n *}-\Delta t I_{D}^{(n-1) *}, \\
I_{R}^{n}-\Delta t I_{R}^{n *}-\Delta t I_{R}^{(n-1) *}, I_{T}^{n}-\Delta t I_{T}^{n *}-\Delta t I_{T}^{(n-1) *}, \\
R^{n}-\Delta t R^{n *}-\Delta t R^{(n-1) *}, D^{n}-\Delta t D^{n *}-\Delta t D^{(n-1) *}, \\
V^{n}-\Delta t V^{n *}-\Delta t V^{(n-1) *}
\end{array}\right) \Delta t
\end{array}\right\}, \\
& I_{D}^{n+1}=I_{D}^{n}+\frac{1-\alpha}{M(\alpha)}\left[\begin{array}{c}
t_{n+1}^{1-\beta} I_{D}^{*}\left(\begin{array}{c}
t_{n+1}, S^{n}+\Delta t S^{n *}, I^{n}+\Delta t I^{n *}, I_{A}^{n}+\Delta t I_{A}^{n *}, \\
I_{D}^{n}+\Delta t I_{D}^{n *}, I_{R}^{n}+\Delta t I_{R}^{n *}, I_{T}^{n}+\Delta t I_{T}^{n *}, \\
R^{n}+\Delta t R^{n *}, D^{n}+\Delta t D^{n *}, V^{n}+\Delta t V^{n *}
\end{array}\right) \\
-t_{n}^{1-\beta} I_{D}^{*}\left(t_{n}, S^{n}, I^{n}, I_{A}^{n}, I_{D}^{n}, I_{R}^{n}, I_{T}^{n}, R^{n}, D^{n}, V^{n}\right)
\end{array}\right] \\
& +\frac{\alpha}{M(\alpha)} \\
& \times\left\{\begin{array}{c}
\frac{23}{12} t_{n}^{1-\beta} I_{D}^{*}\left(t_{n}, S^{n}, I^{n}, I_{A}^{n}, I_{D}^{n}, I_{R}^{n}, I_{T}^{n}, R^{n}, D^{n}, V^{n}\right) \Delta t \\
-\frac{4}{3} t_{n-1}^{1-\beta} I_{D}^{*}\left(\begin{array}{c}
t_{n-1}, S^{n}-\Delta t S^{n *}, I^{n}-\Delta t I^{n *}, I_{A}^{n}-\Delta t I_{A}^{n *}, \\
I_{D}^{n}-\Delta t I_{D}^{n *}, I_{R}^{n}-\Delta t I_{R}^{n *}, I_{T}^{n}-\Delta t I_{T}^{n *}, \\
R^{n}-\Delta t R^{n *}, D^{n}-\Delta t D^{n *}, V^{n}-\Delta t V^{n *}
\end{array}\right) \Delta t \\
+\frac{5}{12} t_{n-2}^{1-\beta} I_{D}^{*}\left(\begin{array}{c}
t_{n-2}, S^{n}-\Delta t S^{n *}-\Delta t S^{(n-1) *}, I^{n}-\Delta t I^{n *}-\Delta t I^{(n-1) *}, \\
I_{A}^{n}-\Delta t I_{A}^{n *}-\Delta t I_{A}^{(n-1) *}, I_{D}^{n}-\Delta t I_{D}^{n *}-\Delta t I_{D}^{(n-1) *}, \\
I_{R}^{n}-\Delta t I_{R}^{n *}-\Delta t I_{R}^{(n-1) *}, I_{T}^{n}-\Delta t I_{T}^{n *}-\Delta t I_{T}^{(n-1) *}, \\
R^{n}-\Delta t R^{n *}-\Delta t R^{(n-1) *}, D^{n}-\Delta t D^{n *}-\Delta t D^{(n-1) *}, \\
V^{n}-\Delta t V^{n *}-\Delta t V^{(n-1) *}
\end{array}\right) \Delta t
\end{array}\right\}, \\
& I_{R}^{n+1}=I_{R}^{n}+\frac{1-\alpha}{M(\alpha)}\left[\begin{array}{c}
t_{n+1}^{1-\beta} I_{R}^{*}\left(\begin{array}{c}
t_{n+1}, S^{n}+\Delta t S^{n *}, I^{n}+\Delta t I^{n *}, I_{A}^{n}+\Delta t I_{A}^{n *}, \\
I_{D}^{n}+\Delta t I_{D}^{n *}, I_{R}^{n}+\Delta t I_{R}^{n *}, I_{T}^{n}+\Delta t I_{T}^{n *}, \\
R^{n}+\Delta t R^{n *}, D^{n}+\Delta t D^{n *}, V^{n}+\Delta t V^{n *}
\end{array}\right) \\
-t_{n}^{1-\beta} I_{R}^{*}\left(t_{n}, S^{n}, I^{n}, I_{A}^{n}, I_{D}^{n}, I_{R}^{n}, I_{T}^{n}, R^{n}, D^{n}, V^{n}\right)
\end{array}\right] \\
& +\frac{\alpha}{M(\alpha)}
\end{aligned}
$$




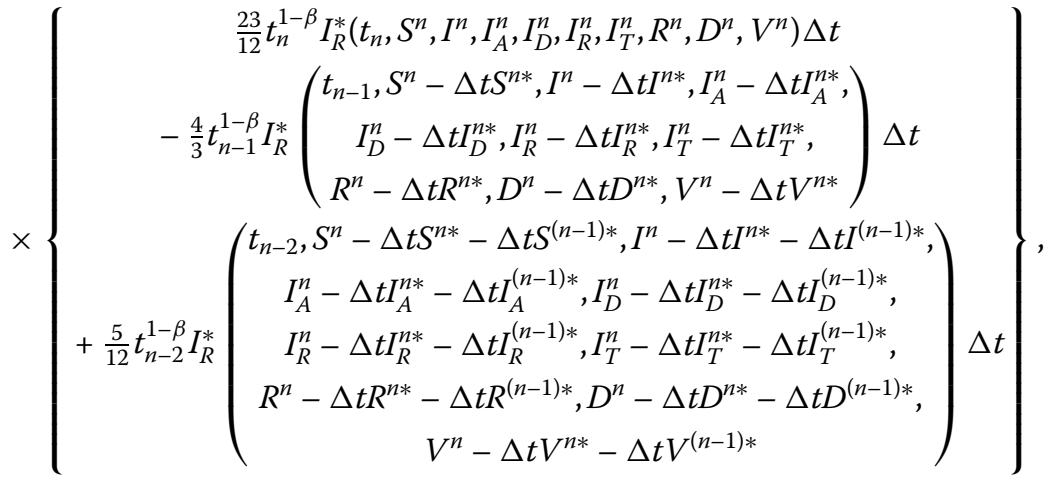

$$
\begin{aligned}
& I_{T}^{n+1}=I_{T}^{n}+\frac{1-\alpha}{M(\alpha)}\left[\begin{array}{c}
t_{n+1}^{1-\beta} I_{T}^{*}\left(\begin{array}{c}
t_{n+1}, S^{n}+\Delta t S^{n *}, I^{n}+\Delta t I^{n *}, I_{A}^{n}+\Delta t I_{A}^{n *}, \\
I_{D}^{n}+\Delta t I_{D}^{n *}, I_{R}^{n}+\Delta t I_{R}^{n *}, I_{T}^{n}+\Delta t I_{T}^{n *}, \\
R^{n}+\Delta t R^{n *}, D^{n}+\Delta t D^{n *}, V^{n}+\Delta t V^{n *}
\end{array}\right) \\
-t_{n}^{1-\beta} I_{T}^{*}\left(t_{n}, S^{n}, I^{n}, I_{A}^{n}, I_{D}^{n}, I_{R}^{n}, I_{T}^{n}, R^{n}, D^{n}, V^{n}\right)
\end{array}\right] \\
& +\frac{\alpha}{M(\alpha)} \\
& \times\left\{\begin{array}{c}
\frac{23}{12} t_{n}^{1-\beta} I_{T}^{*}\left(t_{n}, S^{n}, I^{n}, I_{A}^{n}, I_{D}^{n}, I_{R}^{n}, I_{T}^{n}, R^{n}, D^{n}, V^{n}\right) \Delta t \\
-\frac{4}{3} t_{n-1}^{1-\beta} I_{T}^{*}\left(\begin{array}{c}
t_{n-1}, S^{n}-\Delta t S^{n *}, I^{n}-\Delta t I^{n *}, I_{A}^{n}-\Delta t I_{A}^{n *}, \\
I_{D}^{n}-\Delta t I_{D}^{n *}, I_{R}^{n}-\Delta t I_{R}^{n *}, I_{T}^{n}-\Delta t I_{T}^{n *}, \\
R^{n}-\Delta t R^{n *}, D^{n}-\Delta t D^{n *}, V^{n}-\Delta t V^{n *}
\end{array}\right) \Delta t \\
+\frac{5}{12} t_{n-2}^{1-\beta} I_{T}^{*}\left(\begin{array}{c}
t_{n-2}, S^{n}-\Delta t S^{n *}-\Delta t S^{(n-1) *}, I^{n}-\Delta t I^{n *}-\Delta t I^{(n-1) *} \\
I_{A}^{n}-\Delta t I_{A}^{n *}-\Delta t I_{A}^{(n-1) *}, I_{D}^{n}-\Delta t I_{D}^{n *}-\Delta t I_{D}^{(n-1) *}, \\
I_{R}^{n}-\Delta t I_{R}^{n *}-\Delta t I_{R}^{(n-1) *}, I_{T}^{n}-\Delta t I_{T}^{n *}-\Delta t I_{T}^{(n-1) *}, \\
R^{n}-\Delta t R^{n *}-\Delta t R^{(n-1) *}, D^{n}-\Delta t D^{n *}-\Delta t D^{(n-1) *}, \\
V^{n}-\Delta t V^{n *}-\Delta t V^{(n-1) *}
\end{array}\right\} \Delta t
\end{array}\right\}, \\
& R^{n+1}=R^{n}+\frac{1-\alpha}{M(\alpha)}\left[\begin{array}{c}
t_{n+1}^{1-\beta} R^{*}\left(\begin{array}{c}
t_{n+1}, S^{n}+\Delta t S^{n *}, I^{n}+\Delta t I^{n *}, I_{A}^{n}+\Delta t I_{A}^{n *}, \\
I_{D}^{n}+\Delta t I_{D}^{n *}, I_{R}^{n}+\Delta t I_{R}^{n *}, I_{T}^{n}+\Delta t I_{T}^{n *}, \\
R^{n}+\Delta t R^{n *}, D^{n}+\Delta t D^{n *}, V^{n}+\Delta t V^{n *}
\end{array}\right) \\
-t_{n}^{1-\beta} R^{*}\left(t_{n}, S^{n}, I^{n}, I_{A}^{n}, I_{D}^{n}, I_{R}^{n}, I_{T}^{n}, R^{n}, D^{n}, V^{n}\right)
\end{array}\right] \\
& +\frac{\alpha}{M(\alpha)} \\
& \left.\times\left\{\begin{array}{c}
\frac{23}{12} t_{n}^{1-\beta} R^{*}\left(t_{n}, S^{n}, I^{n}, I_{A}^{n}, I_{D}^{n}, I_{R}^{n}, I_{T}^{n}, R^{n}, D^{n}, V^{n}\right) \Delta t \\
-\frac{4}{3} t_{n-1}^{1-\beta} R^{*}\left(\begin{array}{c}
t_{n-1}, S^{n}-\Delta t S^{n *}, I^{n}-\Delta t I^{n *}, I_{A}^{n}-\Delta t I_{A}^{n *}, \\
I_{D}^{n}-\Delta t I_{D}^{n *}, I_{R}^{n}-\Delta t I_{R}^{n *}, I_{T}^{n}-\Delta t I_{T}^{n *}, \\
R^{n}-\Delta t R^{n *}, D^{n}-\Delta t D^{n *}, V^{n}-\Delta t V^{n *}
\end{array}\right) \Delta t \\
+\frac{5}{12} t_{n-2}^{1-\beta} R^{*}\left(\begin{array}{c}
t_{n-2}, S^{n}-\Delta t S^{n *}-\Delta t S^{(n-1) *}, I^{n}-\Delta t I^{n *}-\Delta t I^{(n-1) *} \\
I_{A}^{n}-\Delta t I_{A}^{n *}-\Delta t I_{A}^{(n-1) *}, I_{D}^{n}-\Delta t I_{D}^{n *}-\Delta t I_{D}^{(n-1) *}, \\
I_{R}^{n}-\Delta t I_{R}^{n *}-\Delta t I_{R}^{(n-1) *}, I_{T}^{n}-\Delta t I_{T}^{n *}-\Delta t I_{T}^{(n-1) *}, \\
R^{n}-\Delta t R^{n *}-\Delta t R^{(n-1) *}, D^{n}-\Delta t D^{n *}-\Delta t D^{(n-1) *}, \\
V^{n}-\Delta t V^{n *}-\Delta t V^{(n-1) *}
\end{array}\right.
\end{array}\right\}, \Delta t\right\}
\end{aligned}
$$




$$
\begin{aligned}
& D^{n+1}=D^{n}+\frac{1-\alpha}{M(\alpha)}\left[\begin{array}{c}
t_{n+1}^{1-\beta} D^{*}\left(\begin{array}{c}
t_{n+1}, S^{n}+\Delta t S^{n *}, I^{n}+\Delta t I^{n *}, I_{A}^{n}+\Delta t I_{A}^{n *}, \\
I_{D}^{n}+\Delta t I_{D}^{n *}, I_{R}^{n}+\Delta t I_{R}^{n *}, I_{T}^{n}+\Delta t I_{T}^{n *}, \\
R^{n}+\Delta t R^{n *}, D^{n}+\Delta t D^{n *}, V^{n}+\Delta t V^{n *}
\end{array}\right) \\
-t_{n}^{1-\beta} D^{*}\left(t_{n}, S^{n}, I^{n}, I_{A}^{n}, I_{D}^{n}, I_{R}^{n}, I_{T}^{n}, R^{n}, D^{n}, V^{n}\right)
\end{array}\right] \\
& +\frac{\alpha}{M(\alpha)} \\
& \times\left\{\begin{array}{c}
\frac{23}{12} t_{n}^{1-\beta} D^{*}\left(t_{n}, S^{n}, I^{n}, I_{A}^{n}, I_{D}^{n}, I_{R}^{n}, I_{T}^{n}, R^{n}, D^{n}, V^{n}\right) \Delta t \\
-\frac{4}{3} t_{n-1}^{1-\beta} D^{*}\left(\begin{array}{c}
t_{n-1}, S^{n}-\Delta t S^{n *}, I^{n}-\Delta t I^{n *}, I_{A}^{n}-\Delta t I_{A}^{n *}, \\
I_{D}^{n}-\Delta t I_{D}^{n *}, I_{R}^{n}-\Delta t I_{R}^{n *}, I_{T}^{n}-\Delta t I_{T}^{n *}, \\
R^{n}-\Delta t R^{n *}, D^{n}-\Delta t D^{n *}, V^{n}-\Delta t V^{n *}
\end{array}\right) \Delta t \\
+\frac{5}{12} t_{n-2}^{1-\beta} D^{*}\left(\begin{array}{c}
t_{n-2}, S^{n}-\Delta t S^{n *}-\Delta t S^{(n-1) *}, I^{n}-\Delta t I^{n *}-\Delta t I^{(n-1) *}, \\
I_{A}^{n}-\Delta t I_{A}^{n *}-\Delta t I_{A}^{(n-1) *}, I_{D}^{n}-\Delta t I_{D}^{n *}-\Delta t I_{D}^{(n-1) *}, \\
I_{R}^{n}-\Delta t I_{R}^{n *}-\Delta t I_{R}^{(n-1) *}, I_{T}^{n}-\Delta t I_{T}^{n *}-\Delta t I_{T}^{(n-1) *}, \\
R^{n}-\Delta t R^{n *}-\Delta t R^{(n-1) *}, D^{n}-\Delta t D^{n *}-\Delta t D^{(n-1) *}, \\
V^{n}-\Delta t V^{n *}-\Delta t V^{(n-1) *}
\end{array}\right\} \Delta t
\end{array}\right\}, \\
& V^{n+1}=V^{n}+\frac{1-\alpha}{M(\alpha)}\left[\begin{array}{c}
t_{n+1}^{1-\beta} V^{*}\left(\begin{array}{c}
t_{n+1}, S^{n}+\Delta t S^{n *}, I^{n}+\Delta t I^{n *}, I_{A}^{n}+\Delta t I_{A}^{n *}, \\
I_{D}^{n}+\Delta t I_{D}^{n *}, I_{R}^{n}+\Delta t I_{R}^{n *}, I_{T}^{n}+\Delta t I_{T}^{n *}, \\
R^{n}+\Delta t R^{n *}, D^{n}+\Delta t D^{n *}, V^{n}+\Delta t V^{n *}
\end{array}\right) \\
-t_{n}^{1-\beta} V^{*}\left(t_{n}, S^{n}, I^{n}, I_{A}^{n}, I_{D}^{n}, I_{R}^{n}, I_{T}^{n}, R^{n}, D^{n}, V^{n}\right)
\end{array}\right] \\
& +\frac{\alpha}{M(\alpha)} \\
& \times\left\{\begin{array}{c}
\frac{23}{12} t_{n}^{1-\beta} V^{*}\left(t_{n}, S^{n}, I^{n}, I_{A}^{n}, I_{D}^{n}, I_{R}^{n}, I_{T}^{n}, R^{n}, D^{n}, V^{n}\right) \Delta t \\
-\frac{4}{3} t_{n-1}^{1-\beta} V^{*}\left(\begin{array}{c}
t_{n-1}, S^{n}-\Delta t S^{n *}, I^{n}-\Delta t I^{n *}, I_{A}^{n}-\Delta t I_{A}^{n *}, \\
I_{D}^{n}-\Delta t I_{D}^{n *}, I_{R}^{n}-\Delta t I_{R}^{n *}, I_{T}^{n}-\Delta t I_{T}^{n *}, \\
R^{n}-\Delta t R^{n *}, D^{n}-\Delta t D^{n *}, V^{n}-\Delta t V^{n *}
\end{array}\right) \Delta t \\
+\frac{5}{12} t_{n-2}^{1-\beta} V^{*}\left(\begin{array}{c}
t_{n-2}, S^{n}-\Delta t S^{n *}-\Delta t S^{(n-1) *}, I^{n}-\Delta t I^{n *}-\Delta t I^{(n-1) *}, \\
I_{A}^{n}-\Delta t I_{A}^{n *}-\Delta t I_{A}^{(n-1) *}, I_{D}^{n}-\Delta t I_{D}^{n *}-\Delta t I_{D}^{(n-1) *}, \\
I_{R}^{n}-\Delta t I_{R}^{n *}-\Delta t I_{R}^{(n-1) *}, I_{T}^{n}-\Delta t I_{T}^{n *}-\Delta t I_{T}^{(n-1) *}, \\
R^{n}-\Delta t R^{n *}-\Delta t R^{(n-1) *}, D^{n}-\Delta t D^{n *}-\Delta t D^{(n-1) *}, \\
V^{n}-\Delta t V^{n *}-\Delta t V^{(n-1) *}
\end{array}\right\} \Delta t
\end{array}\right\} .
\end{aligned}
$$

For the Atangana-Baleanu fractal-fractional derivative, we can have the following numerical scheme:

$$
\begin{aligned}
S^{n+1}= & \frac{1-\alpha}{A B(\alpha)} t_{n+1}^{1-\beta} S^{*}\left(\begin{array}{c}
t_{n+1}, S^{n}+\Delta t S^{n *}, I^{n}+\Delta t I^{n *}, I_{A}^{n}+\Delta t I_{A}^{n *}, \\
I_{D}^{n}+\Delta t I_{D}^{n *}, I_{R}^{n}+\Delta t I_{R}^{n *}, I_{T}^{n}+\Delta t I_{T}^{n *}, \\
R^{n}+\Delta t R^{n *}, D^{n}+\Delta t D^{n *}, V^{n}+\Delta t V^{n *}
\end{array}\right) \\
& +\frac{\alpha(\Delta t)^{\alpha}}{A B(\alpha) \Gamma(\alpha+1)}
\end{aligned}
$$




$$
\begin{aligned}
& \times \sum_{j=2}^{n} t_{j-2}^{1-\beta} S^{*}\left(\begin{array}{c}
t_{j-2}, S^{j}-\Delta t S^{j *}-\Delta t S^{(j-1) *}, I^{j}-\Delta t I^{j *}-\Delta t I^{(j-1) *}, \\
I_{A}^{j}-\Delta t I_{A}^{j *}-\Delta t I_{A}^{(j-1) *}, I_{D}^{j}-\Delta t I_{D}^{j *}-\Delta t I_{D}^{(j-1) *}, \\
I_{R}^{j}-\Delta t I_{R}^{j *}-\Delta t I_{R}^{(j-1) *}, I_{T}^{j}-\Delta t I_{T}^{j *}-\Delta t I_{T}^{(j-1) *}, \\
R^{j}-\Delta t R^{j *}-\Delta t R^{(j-1) *}, D^{j}-\Delta t D^{j *}-\Delta t D^{(j-1) *}, \\
V^{j}-\Delta t V^{j *}-\Delta t V^{(j-1) *}
\end{array}\right) \times \Pi \\
& +\frac{\alpha(\Delta t)^{\alpha}}{A B(\alpha) \Gamma(\alpha+2)}
\end{aligned}
$$

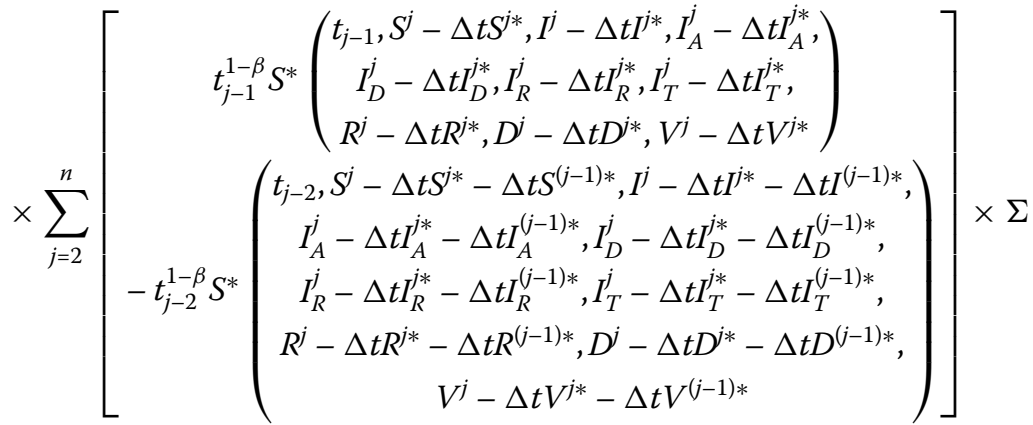

$$
\begin{aligned}
& +\frac{\alpha(\Delta t)^{\alpha}}{2 A B(\alpha) \Gamma(\alpha+3)}
\end{aligned}
$$

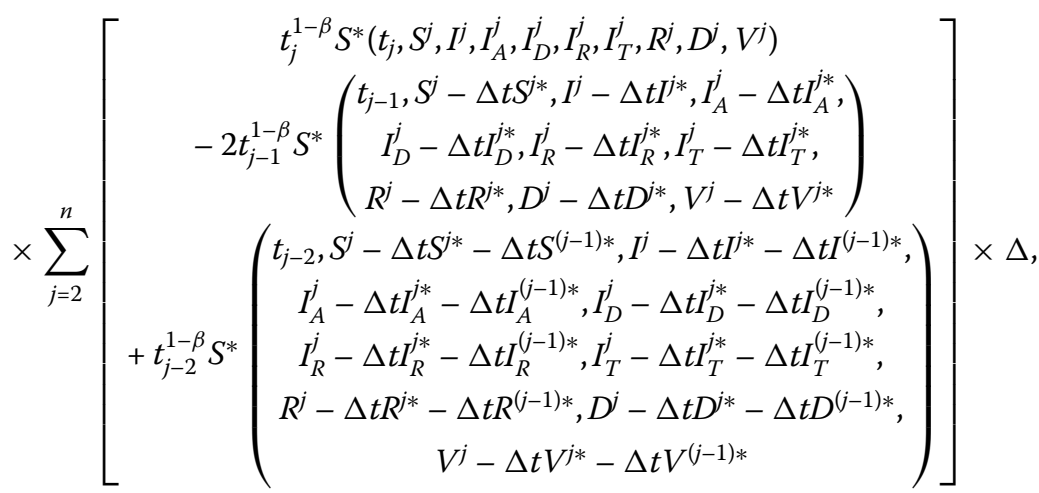

$$
\begin{aligned}
& I^{n+1}=\frac{1-\alpha}{A B(\alpha)} t_{n+1}^{1-\beta} I^{*}\left(\begin{array}{c}
t_{n+1}, S^{n}+\Delta t S^{n *}, I^{n}+\Delta t I^{n *}, I_{A}^{n}+\Delta t I_{A}^{n *}, \\
I_{D}^{n}+\Delta t I_{D}^{n *}, I_{R}^{n}+\Delta t I_{R}^{n *}, I_{T}^{n}+\Delta t I_{T}^{n *}, \\
R^{n}+\Delta t R^{n *}, D^{n}+\Delta t D^{n *}, V^{n}+\Delta t V^{n *}
\end{array}\right) \\
& +\frac{\alpha(\Delta t)^{\alpha}}{A B(\alpha) \Gamma(\alpha+1)} \\
& \times \sum_{j=2}^{n} t_{j-2}^{1-\beta} I^{*}\left(\begin{array}{c}
t_{j-2}, S^{j}-\Delta t S^{j *}-\Delta t S^{(j-1) *}, I^{j}-\Delta t I^{j *}-\Delta t I^{(j-1) *}, \\
I_{A}^{j}-\Delta t I_{A}^{j *}-\Delta t I_{A}^{(j-1) *}, I_{D}^{j}-\Delta t I_{D}^{j *}-\Delta t I_{D}^{(j-1) *}, \\
I_{R}^{j}-\Delta t I_{R}^{j *}-\Delta t I_{R}^{(j-1) *}, I_{T}^{j}-\Delta t I_{T}^{j *}-\Delta t I_{T}^{(j-1) *}, \\
R^{j}-\Delta t R^{j *}-\Delta t R^{(j-1) *}, D^{j}-\Delta t D^{j *}-\Delta t D^{(j-1) *}, \\
V^{j}-\Delta t V^{j *}-\Delta t V^{(j-1) *}
\end{array}\right) \times \Pi \\
& +\frac{\alpha(\Delta t)^{\alpha}}{A B(\alpha) \Gamma(\alpha+2)}
\end{aligned}
$$




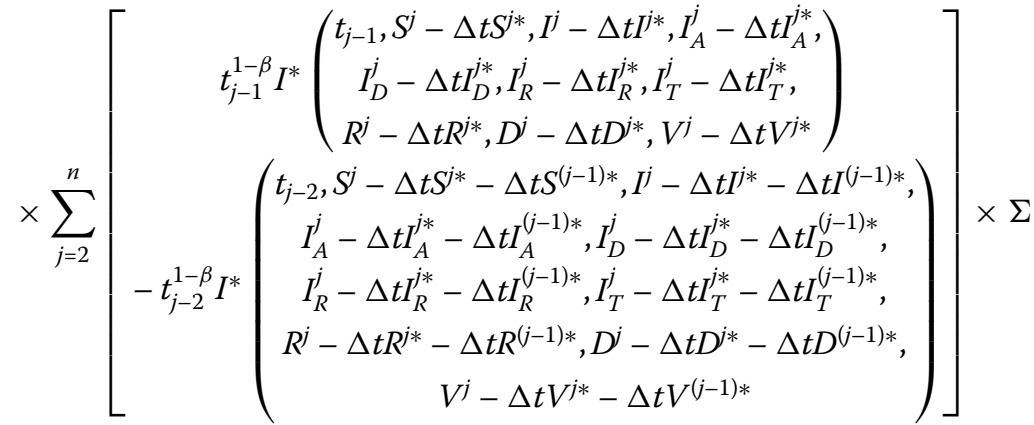

$$
\begin{aligned}
& +\frac{\alpha(\Delta t)^{\alpha}}{2 A B(\alpha) \Gamma(\alpha+3)}
\end{aligned}
$$

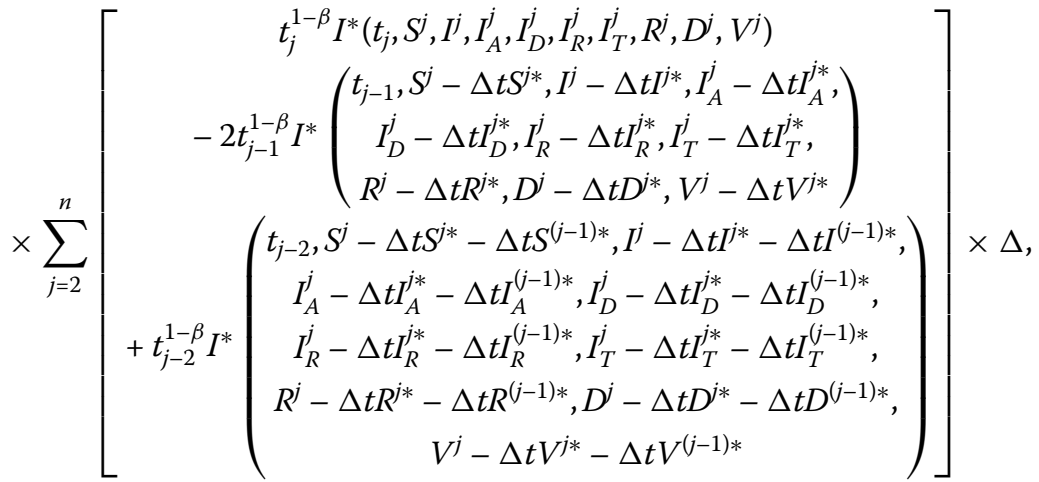

$$
\begin{aligned}
& I_{A}^{n+1}=\frac{1-\alpha}{A B(\alpha)} t_{n+1}^{1-\beta} I_{A}^{*}\left(\begin{array}{c}
t_{n+1}, S^{n}+\Delta t S^{n *}, I^{n}+\Delta t I^{n *}, I_{A}^{n}+\Delta t I_{A}^{n *}, \\
I_{D}^{n}+\Delta t I_{D}^{n *}, I_{R}^{n}+\Delta t I_{R}^{n *}, I_{T}^{n}+\Delta t I_{T}^{n *}, \\
R^{n}+\Delta t R^{n *}, D^{n}+\Delta t D^{n *}, V^{n}+\Delta t V^{n *}
\end{array}\right) \\
& +\frac{\alpha(\Delta t)^{\alpha}}{A B(\alpha) \Gamma(\alpha+1)} \\
& \times \sum_{j=2}^{n} t_{j-2}^{1-\beta} I_{A}^{*}\left(\begin{array}{c}
t_{j-2}, S^{j}-\Delta t S^{j *}-\Delta t S^{(j-1) *}, I^{j}-\Delta t I^{j *}-\Delta t I^{(j-1) *}, \\
I_{A}^{j}-\Delta t I_{A}^{j *}-\Delta t I_{A}^{(j-1) *}, I_{D}^{j}-\Delta t I_{D}^{j *}-\Delta t I_{D}^{(j-1) *}, \\
I_{R}^{j}-\Delta t I_{R}^{j *}-\Delta t I_{R}^{(j-1) *}, I_{T}^{j}-\Delta t I_{T}^{j *}-\Delta t I_{T}^{(j-1) *}, \\
R^{j}-\Delta t R^{j *}-\Delta t R^{(j-1) *}, D^{j}-\Delta t D^{j *}-\Delta t D^{(j-1) *}, \\
V^{j}-\Delta t V^{j *}-\Delta t V^{(j-1) *}
\end{array}\right) \times \Pi \\
& +\frac{\alpha(\Delta t)^{\alpha}}{A B(\alpha) \Gamma(\alpha+2)}
\end{aligned}
$$

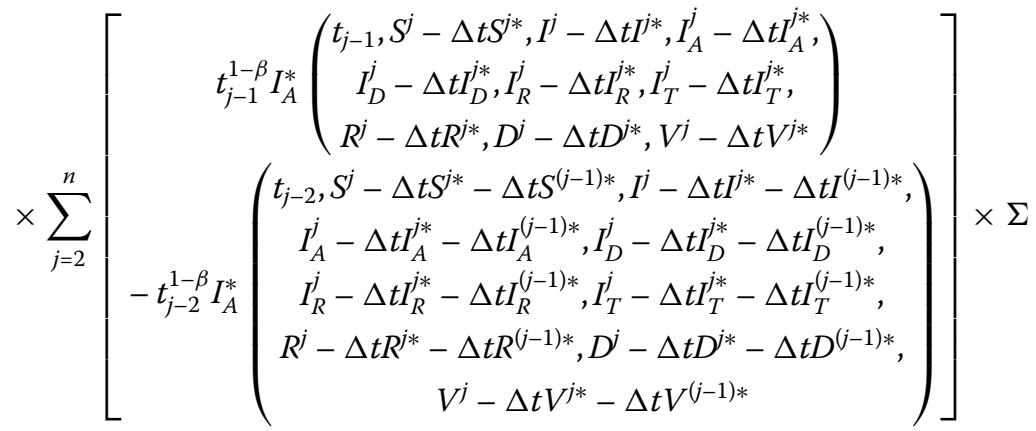

$$
\begin{aligned}
& +\frac{\alpha(\Delta t)^{\alpha}}{2 A B(\alpha) \Gamma(\alpha+3)}
\end{aligned}
$$




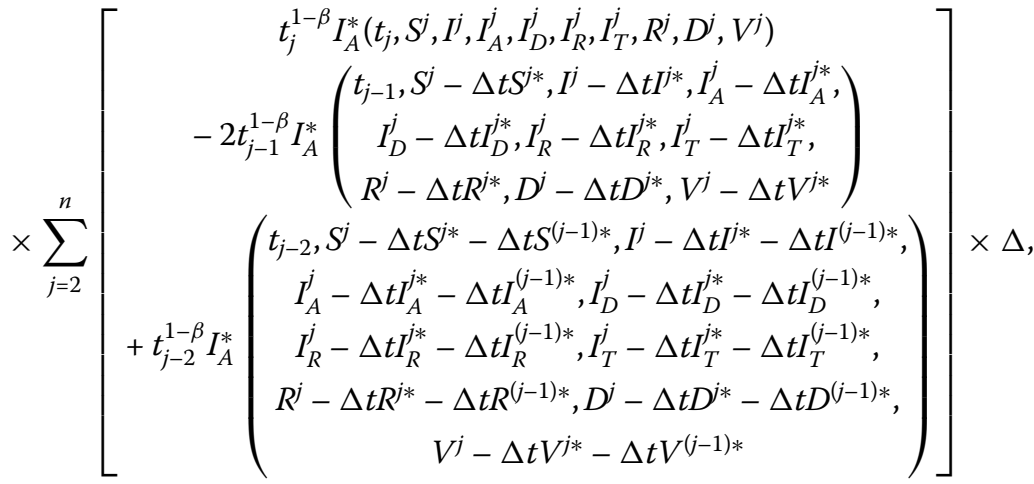

$$
\begin{aligned}
& I_{D}^{n+1}=\frac{1-\alpha}{A B(\alpha)} t_{n+1}^{1-\beta} I_{D}^{*}\left(\begin{array}{c}
t_{n+1}, S^{n}+\Delta t S^{n *}, I^{n}+\Delta t I^{n *}, I_{A}^{n}+\Delta t I_{A}^{n *}, \\
I_{D}^{n}+\Delta t I_{D}^{n *}, I_{R}^{n}+\Delta t I_{R}^{n *}, I_{T}^{n}+\Delta t I_{T}^{n *}, \\
R^{n}+\Delta t R^{n *}, D^{n}+\Delta t D^{n *}, V^{n}+\Delta t V^{n *}
\end{array}\right) \\
& +\frac{\alpha(\Delta t)^{\alpha}}{A B(\alpha) \Gamma(\alpha+1)} \\
& \times \sum_{j=2}^{n} t_{j-2}^{1-\beta} I_{D}^{*}\left(\begin{array}{c}
t_{j-2}, S^{j}-\Delta t S^{j *}-\Delta t S^{(j-1) *}, I^{j}-\Delta t I^{j *}-\Delta t I^{(j-1) *}, \\
I_{A}^{j}-\Delta t I_{A}^{j *}-\Delta t I_{A}^{(j-1) *}, I_{D}^{j}-\Delta t I_{D}^{j *}-\Delta t I_{D}^{(j-1) *}, \\
I_{R}^{j}-\Delta t I_{R}^{j *}-\Delta t I_{R}^{(j-1) *}, I_{T}^{j}-\Delta t I_{T}^{j *}-\Delta t I_{T}^{(j-1) *}, \\
R^{j}-\Delta t R^{j *}-\Delta t R^{(j-1) *}, D^{j}-\Delta t D^{j *}-\Delta t D^{(j-1) *}, \\
V^{j}-\Delta t V^{j *}-\Delta t V^{(j-1) *}
\end{array}\right) \times \Pi \\
& +\frac{\alpha(\Delta t)^{\alpha}}{A B(\alpha) \Gamma(\alpha+2)}
\end{aligned}
$$

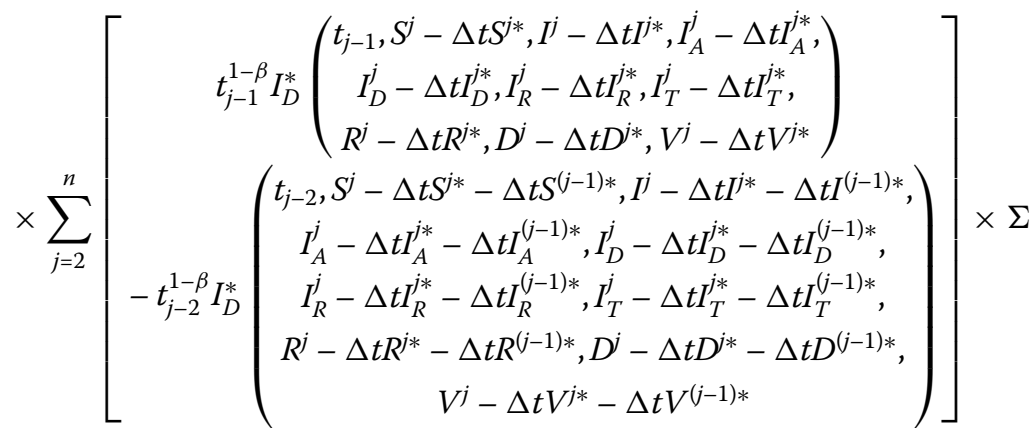

$$
\begin{aligned}
& +\frac{\alpha(\Delta t)^{\alpha}}{2 A B(\alpha) \Gamma(\alpha+3)}
\end{aligned}
$$

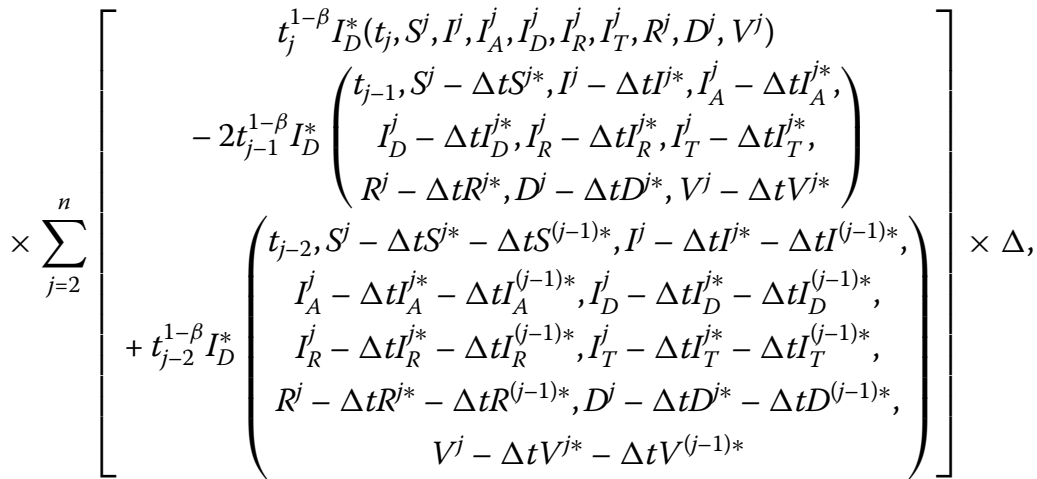




$$
\begin{aligned}
& I_{R}^{n+1}=\frac{1-\alpha}{A B(\alpha)} t_{n+1}^{1-\beta} I_{R}^{*}\left(\begin{array}{c}
t_{n+1}, S^{n}+\Delta t S^{n *}, I^{n}+\Delta t I^{n *}, I_{A}^{n}+\Delta t I_{A}^{n *}, \\
I_{D}^{n}+\Delta t I_{D}^{n *}, I_{R}^{n}+\Delta t I_{R}^{n *}, I_{T}^{n}+\Delta t I_{T}^{n *}, \\
R^{n}+\Delta t R^{n *}, D^{n}+\Delta t D^{n *}, V^{n}+\Delta t V^{n *}
\end{array}\right) \\
& +\frac{\alpha(\Delta t)^{\alpha}}{A B(\alpha) \Gamma(\alpha+1)} \\
& \times \sum_{j=2}^{n} t_{j-2}^{1-\beta} I_{R}^{*}\left(\begin{array}{c}
t_{j-2}, S^{j}-\Delta t S^{j *}-\Delta t S^{(j-1) *}, I^{j}-\Delta t I^{j *}-\Delta t I^{(j-1) *}, \\
I_{A}^{j}-\Delta t I_{A}^{j *}-\Delta t I_{A}^{(j-1) *}, I_{D}^{j}-\Delta t I_{D}^{j *}-\Delta t I_{D}^{(j-1) *}, \\
I_{R}^{j}-\Delta t I_{R}^{j *}-\Delta t I_{R}^{(j-1) *}, I_{T}^{j}-\Delta t I_{T}^{j *}-\Delta t I_{T}^{(j-1) *}, \\
R^{j}-\Delta t R^{j *}-\Delta t R^{(j-1) *}, D^{j}-\Delta t D^{j *}-\Delta t D^{(j-1) *}, \\
V^{j}-\Delta t V^{j *}-\Delta t V^{(j-1) *}
\end{array}\right) \times \Pi \\
& +\frac{\alpha(\Delta t)^{\alpha}}{A B(\alpha) \Gamma(\alpha+2)}
\end{aligned}
$$

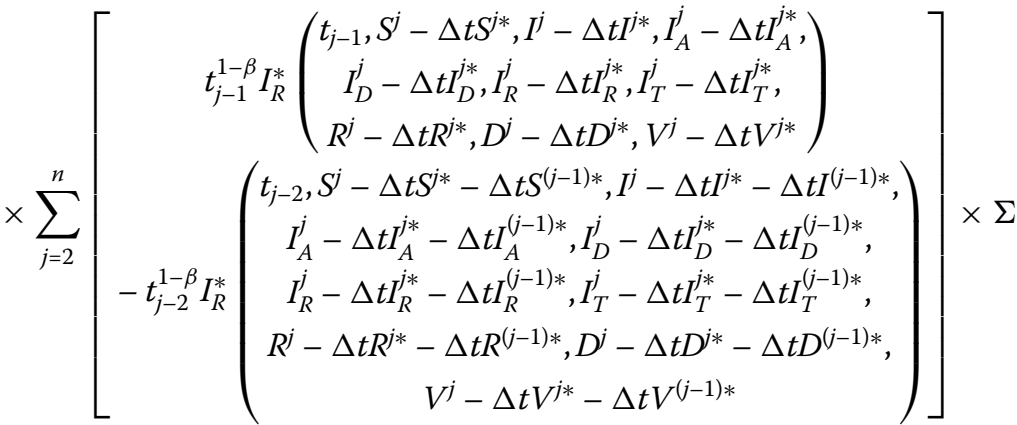

$$
\begin{aligned}
& +\frac{\alpha(\Delta t)^{\alpha}}{2 A B(\alpha) \Gamma(\alpha+3)}
\end{aligned}
$$

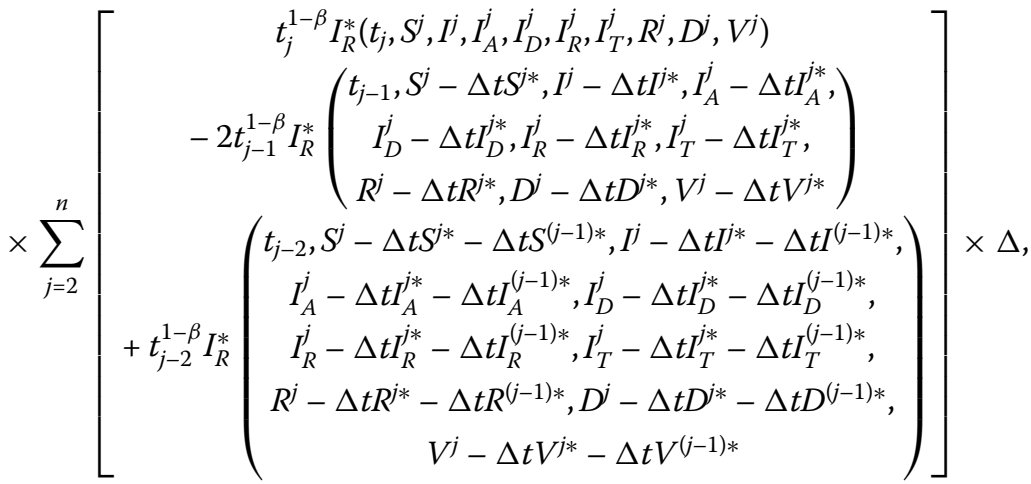

$$
\begin{aligned}
& I_{T}^{n+1}=\frac{1-\alpha}{A B(\alpha)} t_{n+1}^{1-\beta} I_{T}^{*}\left(\begin{array}{c}
t_{n+1}, S^{n}+\Delta t S^{n *}, I^{n}+\Delta t I^{n *}, I_{A}^{n}+\Delta t I_{A}^{n *} \\
I_{D}^{n}+\Delta t I_{D}^{n *}, I_{R}^{n}+\Delta t I_{R}^{n *}, I_{T}^{n}+\Delta t I_{T}^{n *}, \\
R^{n}+\Delta t R^{n *}, D^{n}+\Delta t D^{n *}, V^{n}+\Delta t V^{n *}
\end{array}\right) \\
& +\frac{\alpha(\Delta t)^{\alpha}}{A B(\alpha) \Gamma(\alpha+1)}
\end{aligned}
$$




$$
\begin{aligned}
& \times \sum_{j=2}^{n} t_{j-2}^{1-\beta} I_{T}^{*}\left(\begin{array}{c}
t_{j-2}, S^{j}-\Delta t S^{j *}-\Delta t S^{(j-1) *}, I^{j}-\Delta t I^{j *}-\Delta t I^{(j-1) *}, \\
I_{A}^{j}-\Delta t I_{A}^{j *}-\Delta t I_{A}^{(j-1) *}, I_{D}^{j}-\Delta t I_{D}^{j *}-\Delta t I_{D}^{(j-1) *}, \\
I_{R}^{j}-\Delta t I_{R}^{j *}-\Delta t I_{R}^{(j-1) *}, I_{T}^{j}-\Delta t I_{T}^{j *}-\Delta t I_{T}^{(j-1) *}, \\
R^{j}-\Delta t R^{j *}-\Delta t R^{(j-1) *}, D^{j}-\Delta t D^{j *}-\Delta t D^{(j-1) *}, \\
V^{j}-\Delta t V^{j *}-\Delta t V^{(j-1) *}
\end{array}\right) \times \Pi \\
& +\frac{\alpha(\Delta t)^{\alpha}}{A B(\alpha) \Gamma(\alpha+2)} \\
& \times \sum_{j=2}^{n}\left[\begin{array}{c}
t_{j-1}^{1-\beta} I_{T}^{*}\left(\begin{array}{c}
t_{j-1}, S^{j}-\Delta t S^{*}, I^{j}-\Delta t I^{j *}, I_{A}^{j}-\Delta t I_{A}^{j *}, \\
I_{D}^{j}-\Delta t I_{D}^{j *}, I_{R}^{j}-\Delta t I_{R}^{j *}, I_{T}^{j}-\Delta t I_{T}^{j *}, \\
R^{j}-\Delta t R^{j *}, D^{j}-\Delta t D^{j *}, V^{j}-\Delta t V^{j *}
\end{array}\right) \\
-t_{j-2}^{1-\beta} I_{T}^{*}\left(\begin{array}{c}
t_{j-2}, S^{j}-\Delta t S^{j^{*}}-\Delta t S^{(j-1) *}, I^{j}-\Delta t I^{j *}-\Delta t I^{(j-1) *}, \\
I_{A}^{j}-\Delta t I_{A}^{j *}-\Delta t I_{A}^{(j-1) *}, I_{D}^{j}-\Delta t I_{D}^{j *}-\Delta t I_{D}^{(j-1) *}, \\
I_{R}^{j}-\Delta t I_{R}^{j *}-\Delta t I_{R}^{(j-1) *}, I_{T}^{j}-\Delta t I_{T}^{j *}-\Delta t I_{T}^{(j-1) *}, \\
R^{j}-\Delta t R^{j *}-\Delta t R^{(j-1) *}, D^{j}-\Delta t D^{j *}-\Delta t D^{(j-1) *}, \\
V^{j}-\Delta t V^{j *}-\Delta t V^{(j-1) *}
\end{array}\right)
\end{array}\right] \times \Sigma \\
& +\frac{\alpha(\Delta t)^{\alpha}}{2 A B(\alpha) \Gamma(\alpha+3)} \\
& \times \sum_{j=2}^{n}\left[\begin{array}{c}
t_{j}^{1-\beta} I_{T}^{*}\left(t_{j}, S^{j}, I^{j}, I_{A}^{j}, I_{D}^{j}, I_{R}^{j}, I_{T}^{j}, R^{j}, D^{j}, V^{j}\right) \\
-2 t_{j-12}^{1-\beta} I_{T}^{*}\left(\begin{array}{c}
t_{j-1}, S^{j}-\Delta t S^{j *}, I^{j}-\Delta t I^{j *}, I_{A}^{j}-\Delta t I_{A}^{j *}, \\
I_{D}^{j}-\Delta t I_{D}^{j *}, I_{R}^{j}-\Delta t I_{R}^{j *}, I_{T}^{j}-\Delta t I_{T}^{j *}, \\
R^{j}-\Delta t R^{j *}, D^{j}-\Delta t D^{j *}, V^{j}-\Delta t V^{j *}
\end{array}\right) \\
+t_{j-2}^{1-\beta} I_{T}^{*}\left(\begin{array}{c}
t_{j-2}, S^{j}-\Delta t S^{j *}-\Delta t S^{(j-1) *}, I^{j}-\Delta t I^{j *}-\Delta t I^{(j-1) *} \\
I_{A}^{j}-\Delta t I_{A}^{j *}-\Delta t I_{A}^{(j-1) *}, I_{D}^{j}-\Delta t I_{D}^{j *}-\Delta t I_{D}^{(j-1) *}, \\
I_{R}^{j}-\Delta t I_{R}^{j *}-\Delta t I_{R}^{(j-1) *}, I_{T}^{j}-\Delta t I_{T}^{j *}-\Delta t I_{T}^{(j-1) *}, \\
R^{j}-\Delta t R^{j *}-\Delta t R^{(j-1) *}, D^{j}-\Delta t D^{j *}-\Delta t D^{(j-1) *}, \\
V^{j}-\Delta t V^{j *}-\Delta t V^{(j-1) *}
\end{array}\right)
\end{array}\right] \times \Delta, \\
& R^{n+1}=\frac{1-\alpha}{A B(\alpha)} t_{n+1}^{1-\beta} R^{*}\left(\begin{array}{c}
t_{n+1}, S^{n}+\Delta t S^{n *}, I^{n}+\Delta t I^{n *}, I_{A}^{n}+\Delta t I_{A}^{n *}, \\
I_{D}^{n}+\Delta t I_{D}^{n *}, I_{R}^{n}+\Delta t I_{R}^{n *}, I_{T}^{n}+\Delta t I_{T}^{n *}, \\
R^{n}+\Delta t R^{n *}, D^{n}+\Delta t D^{n *}, V^{n}+\Delta t V^{n *}
\end{array}\right) \\
& +\frac{\alpha(\Delta t)^{\alpha}}{A B(\alpha) \Gamma(\alpha+1)} \\
& \times \sum_{j=2}^{n} t_{j-2}^{1-\beta} R^{*}\left(\begin{array}{c}
t_{j-2}, S^{j}-\Delta t S^{j *}-\Delta t S^{(j-1) *}, I^{j}-\Delta t I^{j *}-\Delta t I^{(j-1) *}, \\
I_{A}^{j}-\Delta t I_{A}^{j *}-\Delta t I_{A}^{(j-1) *}, I_{D}^{j}-\Delta t I_{D}^{j *}-\Delta t I_{D}^{(j-1) *}, \\
I_{R}^{j}-\Delta t I_{R}^{j *}-\Delta t I_{R}^{(j-1) *}, I_{T}^{j}-\Delta t I_{T}^{j *}-\Delta t I_{T}^{(j-1) *}, \\
R^{j}-\Delta t R^{j *}-\Delta t R^{(j-1) *}, D^{j}-\Delta t D^{j *}-\Delta t D^{(j-1) *}, \\
V^{j}-\Delta t V^{j *}-\Delta t V^{(j-1) *}
\end{array}\right) \times \Pi \\
& +\frac{\alpha(\Delta t)^{\alpha}}{A B(\alpha) \Gamma(\alpha+2)}
\end{aligned}
$$




$$
\begin{aligned}
& \times \sum_{j=2}^{n}\left[\begin{array}{c}
t_{j-1}, S^{j}-\Delta t S^{j *}, I^{j}-\Delta t I^{j *}, I_{A}^{j}-\Delta t I_{A}^{j *}, \\
I_{D}^{j}-\Delta t I_{D}^{j *}, I_{R}^{j}-\Delta t I_{R}^{j^{*}}, I_{T}^{j}-\Delta t I_{T}^{j *}, \\
R^{j}-\Delta t R^{j *}, D^{j}-\Delta t D^{j *}, V^{j}-\Delta t V^{j *}
\end{array}\right)\left(\begin{array}{c}
t_{j-2}, S^{j}-\Delta t S^{j *}-\Delta t S^{(j-1) *}, I^{j}-\Delta t I^{j *}-\Delta t I^{(j-1) *}, \\
I_{A}^{j}-\Delta t I_{A}^{j *}-\Delta t I_{A}^{(j-1) *}, I_{D}^{j}-\Delta t I_{D}^{j *}-\Delta t I_{D}^{(j-1) *}, \\
I_{R}^{j}-\Delta t I_{R}^{j *}-\Delta t I_{R}^{(j-1) *}, I_{T}^{j}-\Delta t I_{T}^{j *}-\Delta t I_{T}^{(j-1) *}, \\
R^{j}-\Delta t R^{j *}-\Delta t R^{(j-1) *}, D^{j}-\Delta t D^{j *}-\Delta t D^{(j-1) *}, \\
V^{j}-\Delta t V^{j *}-\Delta t V^{(j-1) *}
\end{array}\right) \times \Sigma \\
& +\frac{\alpha(\Delta t)^{\alpha}}{2 A B(\alpha) \Gamma(\alpha+3)}
\end{aligned}
$$

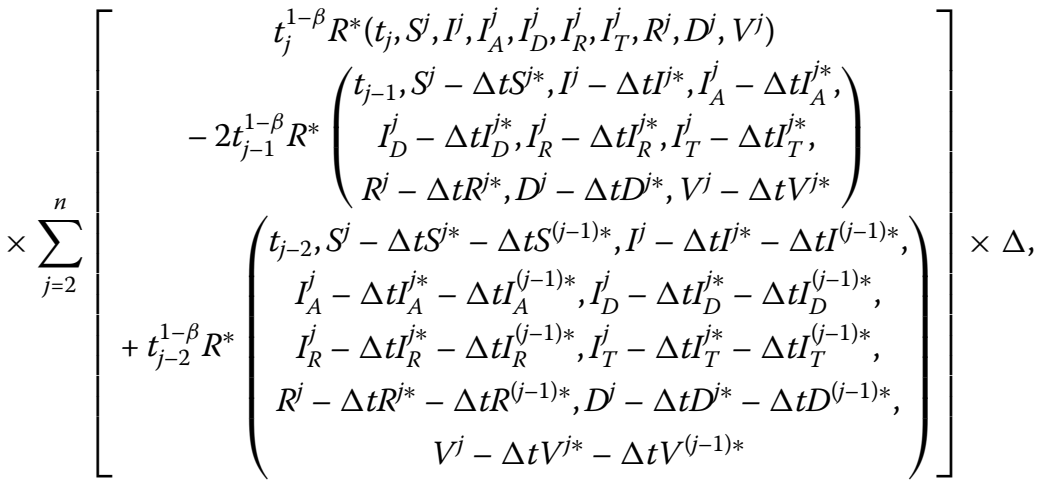

$$
\begin{aligned}
& D^{n+1}=\frac{1-\alpha}{A B(\alpha)} t_{n+1}^{1-\beta} D^{*}\left(\begin{array}{c}
t_{n+1}, S^{n}+\Delta t S^{n *}, I^{n}+\Delta t I^{n *}, I_{A}^{n}+\Delta t I_{A}^{n *}, \\
I_{D}^{n}+\Delta t I_{D}^{n *}, I_{R}^{n}+\Delta t I_{R}^{n *}, I_{T}^{n}+\Delta t I_{T}^{n *}, \\
R^{n}+\Delta t R^{n *}, D^{n}+\Delta t D^{n *}, V^{n}+\Delta t V^{n *}
\end{array}\right) \\
& +\frac{\alpha(\Delta t)^{\alpha}}{A B(\alpha) \Gamma(\alpha+1)} \\
& \times \sum_{j=2}^{n} t_{j-2}^{1-\beta} D^{*}\left(\begin{array}{c}
t_{j-2}, S^{j}-\Delta t S^{j *}-\Delta t S^{(j-1) *}, I^{j}-\Delta t I^{j *}-\Delta t I^{(j-1) *}, \\
I_{A}^{j}-\Delta t I_{A}^{j *}-\Delta t I_{A}^{(j-1) *}, I_{D}^{j}-\Delta t I_{D}^{j *}-\Delta t I_{D}^{(j-1) *}, \\
I_{R}^{j}-\Delta t I_{R}^{j *}-\Delta t I_{R}^{(j-1) *}, I_{T}^{j}-\Delta t I_{T}^{j *}-\Delta t I_{T}^{(j-1) *}, \\
R^{j}-\Delta t R^{j *}-\Delta t R^{(j-1) *}, D^{j}-\Delta t D^{j *}-\Delta t D^{(j-1) *}, \\
V^{j}-\Delta t V^{j *}-\Delta t V^{(j-1) *}
\end{array}\right) \times \Pi \\
& +\frac{\alpha(\Delta t)^{\alpha}}{A B(\alpha) \Gamma(\alpha+2)}
\end{aligned}
$$

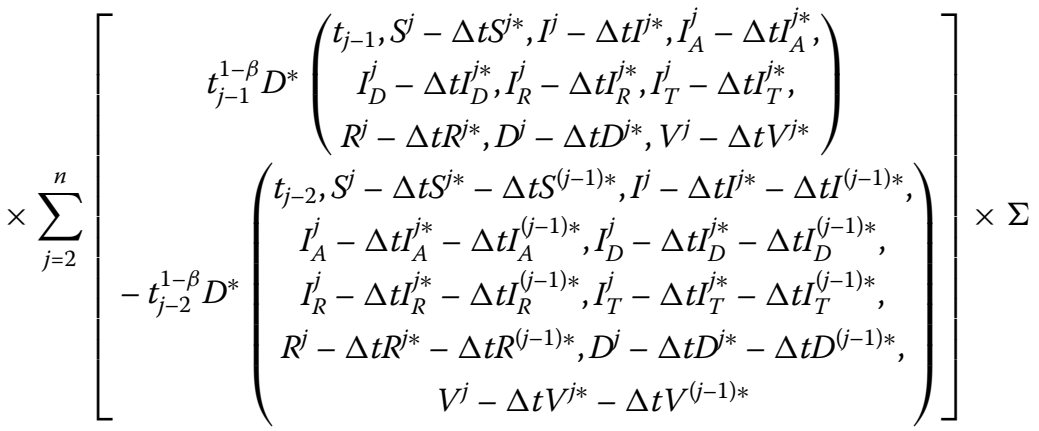

$$
\begin{aligned}
& +\frac{\alpha(\Delta t)^{\alpha}}{2 A B(\alpha) \Gamma(\alpha+3)}
\end{aligned}
$$




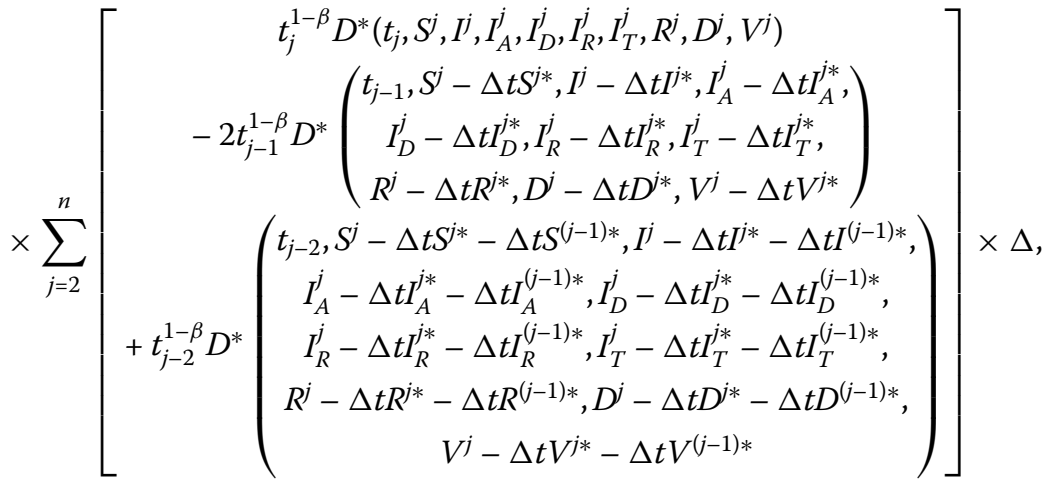

$$
\begin{aligned}
& V^{n+1}=\frac{1-\alpha}{A B(\alpha)} t_{n+1}^{1-\beta} V^{*}\left(\begin{array}{c}
t_{n+1}, S^{n}+\Delta t S^{n *}, I^{n}+\Delta t I^{n *}, I_{A}^{n}+\Delta t I_{A}^{n *}, \\
I_{D}^{n}+\Delta t I_{D}^{n *}, I_{R}^{n}+\Delta t I_{R}^{n *}, I_{T}^{n}+\Delta t I_{T}^{n *}, \\
R^{n}+\Delta t R^{n *}, D^{n}+\Delta t D^{n *}, V^{n}+\Delta t V^{n *}
\end{array}\right) \\
& +\frac{\alpha(\Delta t)^{\alpha}}{A B(\alpha) \Gamma(\alpha+1)} \\
& \times \sum_{j=2}^{n} t_{j-2}^{1-\beta} V^{*}\left(\begin{array}{c}
t_{j-2}, S^{j}-\Delta t S^{j *}-\Delta t S^{(j-1) *}, I^{j}-\Delta t I^{j *}-\Delta t I^{(j-1) *}, \\
I_{A}^{j}-\Delta t I_{A}^{j *}-\Delta t I_{A}^{(j-1) *}, I_{D}^{j}-\Delta t I_{D}^{j *}-\Delta t I_{D}^{(j-1) *}, \\
I_{R}^{j}-\Delta t I_{R}^{j *}-\Delta t I_{R}^{(j-1) *}, I_{T}^{j}-\Delta t I_{T}^{j *}-\Delta t I_{T}^{(j-1) *}, \\
R^{j}-\Delta t R^{j *}-\Delta t R^{(j-1) *}, D^{j}-\Delta t D^{j *}-\Delta t D^{(j-1) *}, \\
V^{j}-\Delta t V^{j *}-\Delta t V^{(j-1) *}
\end{array}\right) \times \Pi \\
& +\frac{\alpha(\Delta t)^{\alpha}}{A B(\alpha) \Gamma(\alpha+2)} \\
& \times \sum_{j=2}^{n}\left[\begin{array}{c}
t_{j-1}, S^{j}-\Delta t S^{j *}, I^{j}-\Delta t I^{j *}, I_{A}^{j}-\Delta t I_{A}^{j *}, \\
t_{D-1}^{j-\beta} V^{*}-\Delta t I_{D}^{j *}, I_{R}^{j}-\Delta t I_{R}^{j *}, I_{T}^{j}-\Delta t I_{T}^{j *}, \\
R^{j}-\Delta t R^{j *}, D^{j}-\Delta t D^{j *}, V^{j}-\Delta t V^{j *}
\end{array}\right)\left(\begin{array}{c}
t_{j-2}, S^{j}-\Delta t S^{j *}-\Delta t S^{(j-1) *}, I^{j}-\Delta t I^{j *}-\Delta t I^{(j-1) *}, \\
I_{A}^{j}-\Delta t I_{A}^{j *}-\Delta t I_{A}^{(j-1) *}, I_{D}^{j}-\Delta t I_{D}^{j *}-\Delta t I_{D}^{(j-1) *}, \\
I_{R}^{j}-\Delta t I_{R}^{j *}-\Delta t I_{R}^{(j-1) *}, I_{T}^{j}-\Delta t I_{T}^{j *}-\Delta t I_{T}^{(j-1) *}, \\
R^{j}-\Delta t R^{j *}-\Delta t R^{(j-1) *}, D^{j}-\Delta t D^{j *}-\Delta t D^{(j-1) *}, \\
V^{j}-\Delta t V^{j *}-\Delta t V^{(j-1) *}
\end{array}\right) \times \Sigma \\
& +\frac{\alpha(\Delta t)^{\alpha}}{2 A B(\alpha) \Gamma(\alpha+3)}
\end{aligned}
$$

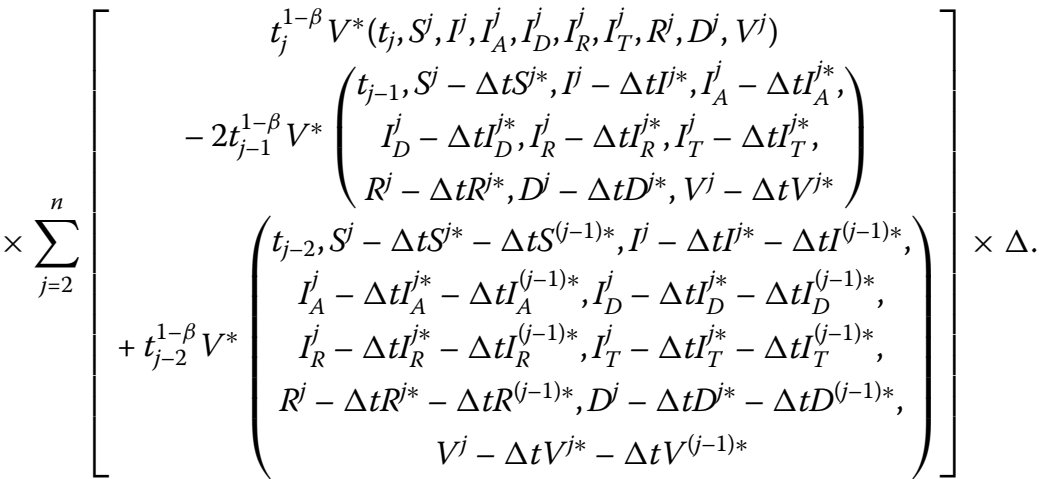


For the power-law kernel, we can have the following:

$$
\begin{aligned}
& S^{n+1}=\frac{(\Delta t)^{\alpha}}{\Gamma(\alpha+1)} \\
& \times \sum_{j=2}^{n} t_{j-2}^{1-\beta} S^{*}\left(\begin{array}{c}
t_{j-2}, S^{j}-\Delta t S^{j *}-\Delta t S^{(j-1) *}, I^{j}-\Delta t I^{j *}-\Delta t I^{(j-1) *}, \\
I_{A}^{j}-\Delta t I_{A}^{j *}-\Delta t I_{A}^{(j-1) *}, I_{D}^{j}-\Delta t I_{D}^{j *}-\Delta t I_{D}^{(j-1) *}, \\
I_{R}^{j}-\Delta t I_{R}^{j *}-\Delta t I_{R}^{(j-1) *}, I_{T}^{j}-\Delta t I_{T}^{j *}-\Delta t I_{T}^{(j-1) *}, \\
R^{j}-\Delta t R^{j *}-\Delta t R^{(j-1) *}, D^{j}-\Delta t D^{j *}-\Delta t D^{(j-1) *}, \\
V^{j}-\Delta t V^{j *}-\Delta t V^{(j-1) *}
\end{array}\right) \times \Pi \\
& +\frac{(\Delta t)^{\alpha}}{\Gamma(\alpha+2)}
\end{aligned}
$$

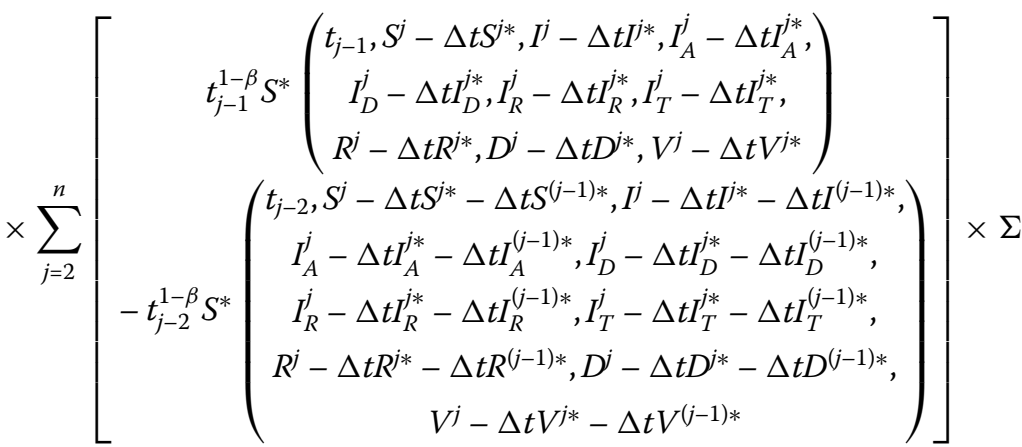

$$
\begin{aligned}
& +\frac{(\Delta t)^{\alpha}}{2 \Gamma(\alpha+3)}
\end{aligned}
$$

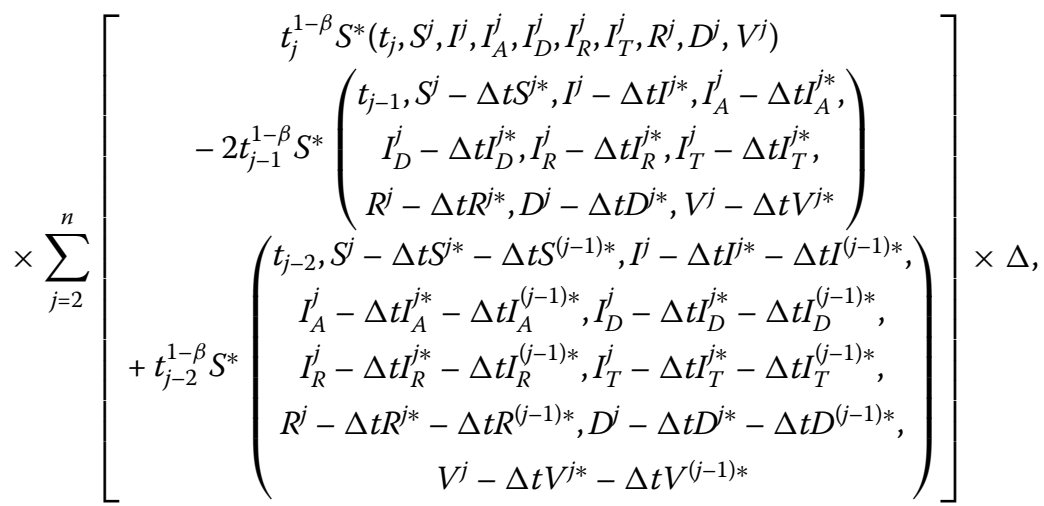

$$
\begin{aligned}
& I^{n+1}=\frac{(\Delta t)^{\alpha}}{\Gamma(\alpha+1)} \sum_{j=2}^{n} t_{j-2}^{1-\beta} I^{*}\left(\begin{array}{c}
t_{j-2}, S^{j}-\Delta t S^{j *}-\Delta t S^{(j-1) *}, I^{j}-\Delta t I^{j *}-\Delta t I^{(j-1) *}, \\
I_{A}^{j}-\Delta t I_{A}^{j *}-\Delta t I_{A}^{(j-1) *}, I_{D}^{j}-\Delta t I_{D}^{j *}-\Delta t I_{D}^{(j-1) *}, \\
I_{R}^{j}-\Delta t I_{R}^{j *}-\Delta t I_{R}^{(j-1) *}, I_{T}^{j}-\Delta t I_{T}^{j *}-\Delta t I_{T}^{(j-1) *}, \\
R^{j}-\Delta t R^{j *}-\Delta t R^{(j-1) *}, D^{j}-\Delta t D^{j *}-\Delta t D^{(j-1) *}, \\
V^{j}-\Delta t V^{j *}-\Delta t V^{(j-1) *}
\end{array}\right) \times \Pi \\
& +\frac{(\Delta t)^{\alpha}}{\Gamma(\alpha+2)}
\end{aligned}
$$




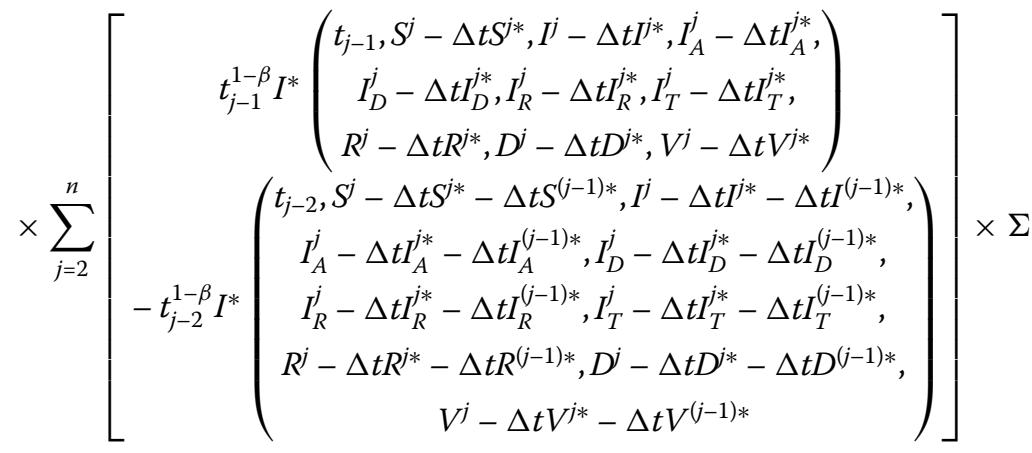

$$
\begin{aligned}
& +\frac{(\Delta t)^{\alpha}}{2 \Gamma(\alpha+3)}
\end{aligned}
$$

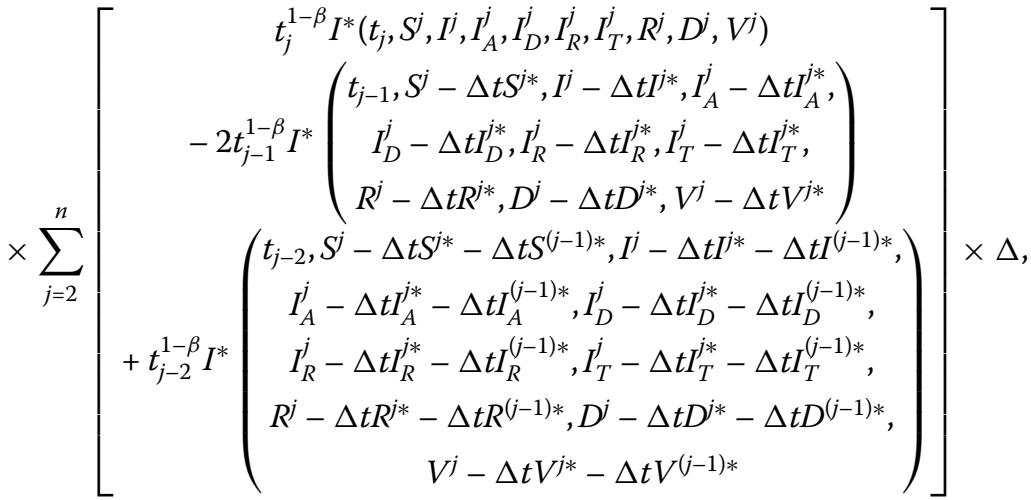

$$
\begin{aligned}
& I_{A}^{n+1}=\frac{(\Delta t)^{\alpha}}{\Gamma(\alpha+1)} \sum_{j=2}^{n} t_{j-2}^{1-\beta} I_{A}^{*}\left(\begin{array}{c}
t_{j-2}, S^{j}-\Delta t S^{j *}-\Delta t S^{(j-1) *}, I^{j}-\Delta t I^{j *}-\Delta t I^{(j-1) *}, \\
I_{A}^{j}-\Delta t I_{A}^{j *}-\Delta t I_{A}^{(j-1) *}, I_{D}^{j}-\Delta t I_{D}^{j *}-\Delta t I_{D}^{(j-1) *}, \\
I_{R}^{j}-\Delta t I_{R}^{j *}-\Delta t I_{R}^{(j-1) *}, I_{T}^{j}-\Delta t I_{T}^{j *}-\Delta t I_{T}^{(j-1) *}, \\
R^{j}-\Delta t R^{j *}-\Delta t R^{(j-1) *}, D^{j}-\Delta t D^{j *}-\Delta t D^{(j-1) *}, \\
V^{j}-\Delta t V^{j *}-\Delta t V^{(j-1) *}
\end{array}\right) \times \Pi \\
& +\frac{(\Delta t)^{\alpha}}{\Gamma(\alpha+2)}
\end{aligned}
$$

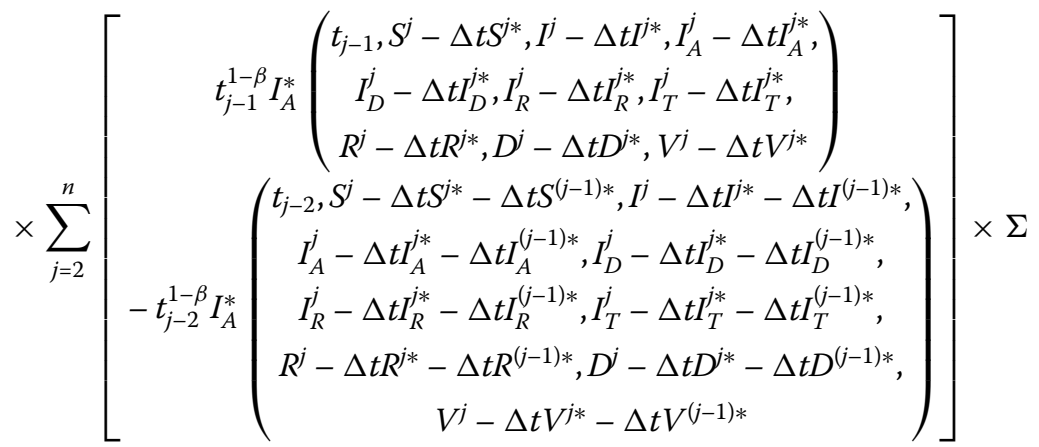

$$
\begin{aligned}
& +\frac{(\Delta t)^{\alpha}}{2 \Gamma(\alpha+3)}
\end{aligned}
$$




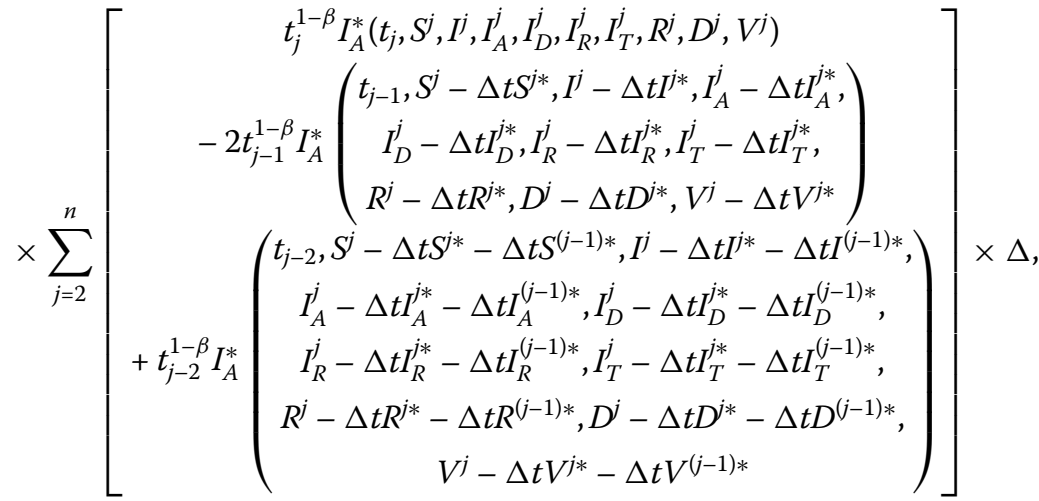

$$
\begin{aligned}
& I_{D}^{n+1}=\frac{(\Delta t)^{\alpha}}{\Gamma(\alpha+1)} \sum_{j=2}^{n} t_{j-2}^{1-\beta} I_{D}^{*}\left(\begin{array}{c}
t_{j-2}, S^{j}-\Delta t S^{j *}-\Delta t S^{(j-1) *}, I^{j}-\Delta t I^{j *}-\Delta t I^{(j-1) *}, \\
I_{A}^{j}-\Delta t I_{A}^{j *}-\Delta t I_{A}^{(j-1) *}, I_{D}^{j}-\Delta t I_{D}^{j *}-\Delta t I_{D}^{(j-1) *}, \\
I_{R}^{j}-\Delta t I_{R}^{j *}-\Delta t I_{R}^{(j-1) *}, I_{T}^{j}-\Delta t I_{T}^{j *}-\Delta t I_{T}^{(j-1) *}, \\
R^{j}-\Delta t R^{j *}-\Delta t R^{(j-1) *}, D^{j}-\Delta t D^{j *}-\Delta t D^{(j-1) *}, \\
V^{j}-\Delta t V^{j *}-\Delta t V^{(j-1) *}
\end{array}\right) \times \Pi \\
& +\frac{(\Delta t)^{\alpha}}{\Gamma(\alpha+2)}
\end{aligned}
$$

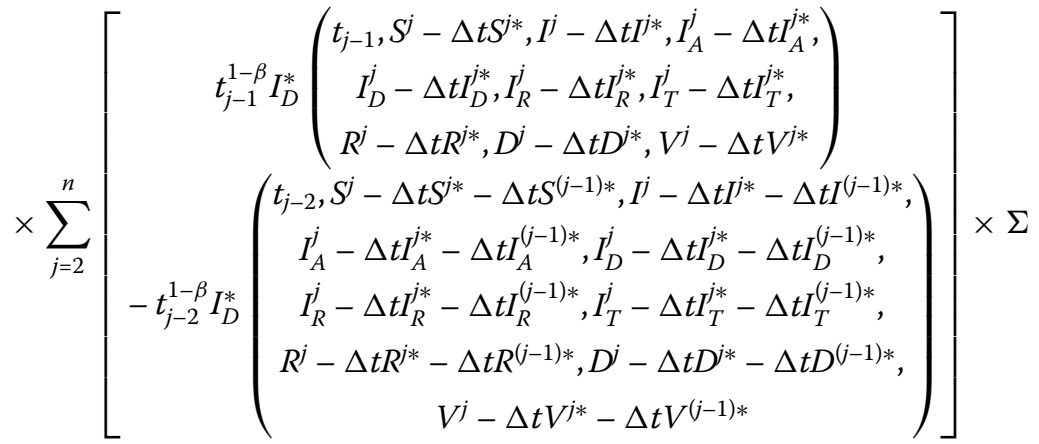

$$
\begin{aligned}
& +\frac{(\Delta t)^{\alpha}}{2 \Gamma(\alpha+3)}
\end{aligned}
$$

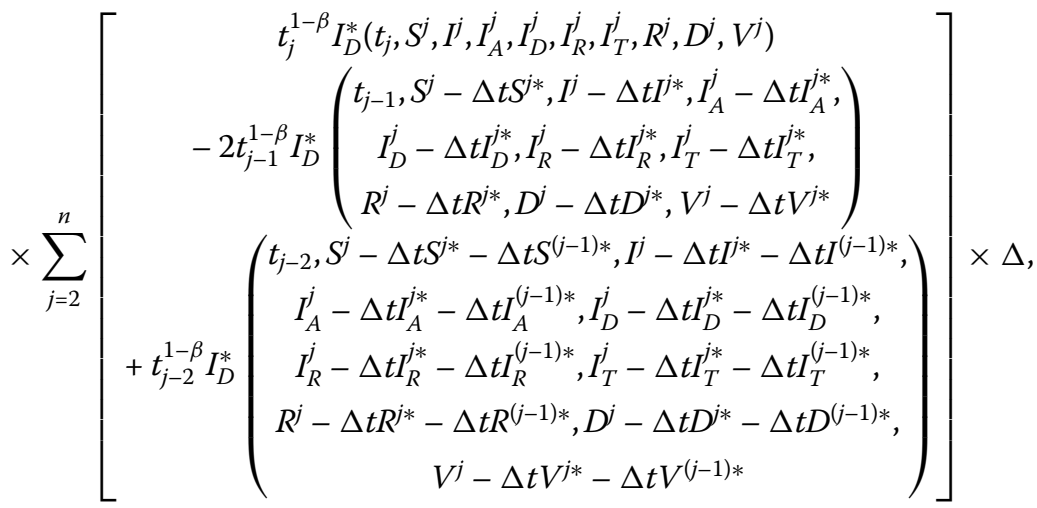




$$
\begin{aligned}
& I_{R}^{n+1}=\frac{(\Delta t)^{\alpha}}{\Gamma(\alpha+1)} \sum_{j=2}^{n} t_{j-2}^{1-\beta} I_{R}^{*}\left(\begin{array}{c}
t_{j-2}, S^{j}-\Delta t S^{j *}-\Delta t S^{(j-1) *}, I^{j}-\Delta t I^{j *}-\Delta t I^{(j-1) *}, \\
I_{A}^{j}-\Delta t I_{A}^{j *}-\Delta t I_{A}^{(j-1) *}, I_{D}^{j}-\Delta t I_{D}^{j *}-\Delta t I_{D}^{(j-1) *}, \\
I_{R}^{j}-\Delta t I_{R}^{j *}-\Delta t I_{R}^{(j-1) *}, I_{T}^{j}-\Delta t I_{T}^{j *}-\Delta t I_{T}^{(j-1) *}, \\
R^{j}-\Delta t R^{j *}-\Delta t R^{(j-1) *}, D^{j}-\Delta t D^{j *}-\Delta t D^{(j-1) *}, \\
V^{j}-\Delta t V^{j *}-\Delta t V^{(j-1) *}
\end{array}\right) \times \Pi \\
& +\frac{(\Delta t)^{\alpha}}{\Gamma(\alpha+2)}\left[\begin{array}{c}
t_{j-12}^{1-\beta} I_{R}^{*}\left(\begin{array}{c}
t_{j-1}, S^{j}-\Delta t S^{j *}, I^{j}-\Delta t I^{j *}, I_{A}^{j}-\Delta t I_{A}^{j *}, I_{D}^{j}-\Delta t I_{D}^{j *}, \\
I_{R}^{j}-\Delta t I_{R}^{j *}, I_{T}^{j}-\Delta t I_{T}^{j *}, R^{j}-\Delta t R^{j *}, D^{j}-\Delta t D^{j *}, V^{j}-\Delta t V^{j *}
\end{array}\right) \\
\times \sum_{j=2}^{n}\left[\begin{array}{c}
t_{j-2}, S^{j}-\Delta t S^{j *}-\Delta t S^{(j-1) *}, I^{j}-\Delta t I^{j *}-\Delta t I^{(j-1) *}, \\
I_{A}^{j}-\Delta t I_{A}^{j *}-\Delta t I_{A}^{(j-1) *}, I_{D}^{j}-\Delta t I_{D}^{j *}-\Delta t I_{D}^{(j-1) *}, \\
I_{R}^{j}-\Delta t I_{R}^{j *}-\Delta t I_{R}^{(j-1) *}, I_{T}^{j}-\Delta t I_{T}^{j *}-\Delta t I_{T}^{(j-1) *}, \\
R^{j}-\Delta t R^{j *}-\Delta t R^{(j-1) *}, D^{j}-\Delta t D^{j *}-\Delta t D^{(j-1) *}, \\
V^{j}-\Delta t V^{j *}-\Delta t V^{(j-1) *}
\end{array}\right)
\end{array}\right]
\end{aligned}
$$

$\times \Sigma$

$+\frac{(\Delta t)^{\alpha}}{2 \Gamma(\alpha+3)}$

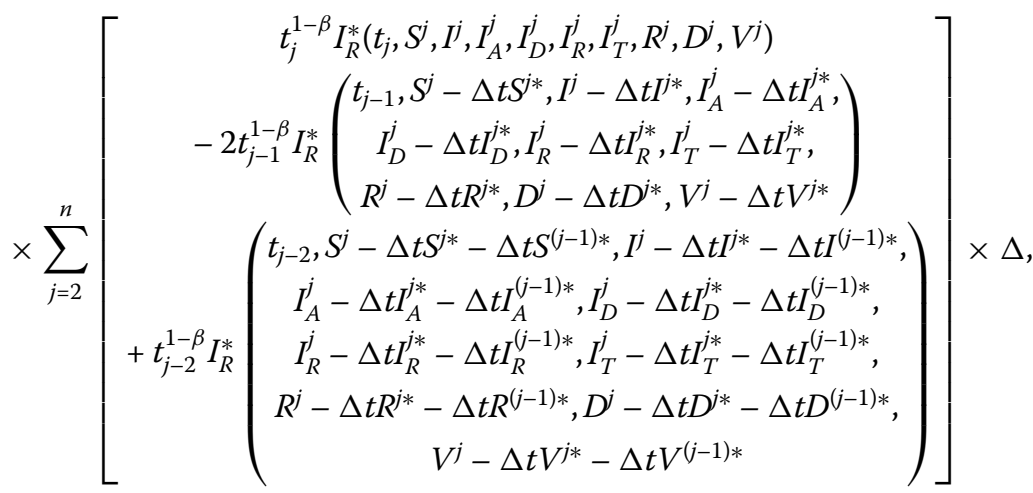

$$
\begin{aligned}
& I_{T}^{n+1}=\frac{(\Delta t)^{\alpha}}{\Gamma(\alpha+1)} \sum_{j=2}^{n} t_{j-2}^{1-\beta} I_{T}^{*}\left(\begin{array}{c}
t_{j-2}, S^{j}-\Delta t S^{j *}-\Delta t S^{(j-1) *}, I^{j}-\Delta t I^{j *}-\Delta t I^{(j-1) *}, \\
I_{A}^{j}-\Delta t I_{A}^{j *}-\Delta t I_{A}^{(j-1) *}, I_{D}^{j}-\Delta t I_{D}^{j *}-\Delta t I_{D}^{(j-1) *}, \\
I_{R}^{j}-\Delta t I_{R}^{j *}-\Delta t I_{R}^{(j-1) *}, I_{T}^{j}-\Delta t I_{T}^{j *}-\Delta t I_{T}^{(j-1) *}, \\
R^{j}-\Delta t R^{j *}-\Delta t R^{(j-1) *}, D^{j}-\Delta t D^{j *}-\Delta t D^{(j-1) *}, \\
V^{j}-\Delta t V^{j *}-\Delta t V^{(j-1) *}
\end{array}\right) \times \Pi \\
& +\frac{\alpha(\Delta t)^{\alpha}}{\Gamma(\alpha+2)}
\end{aligned}
$$




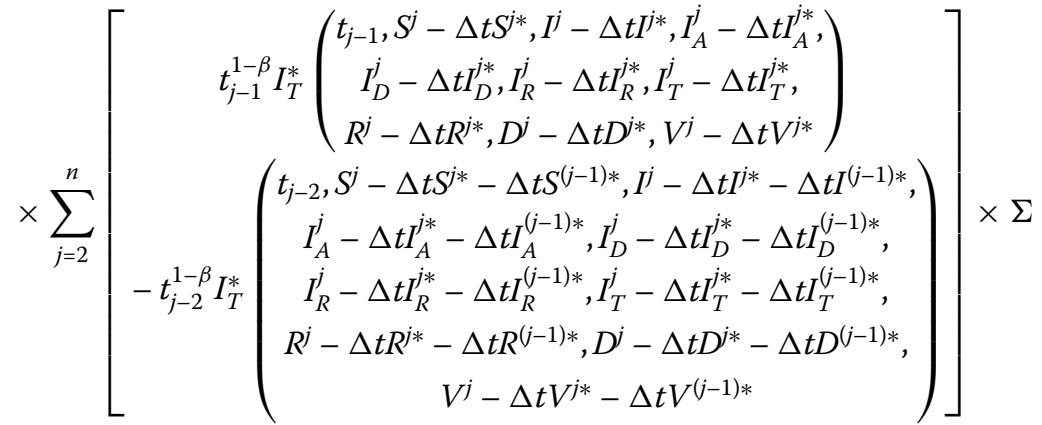

$$
\begin{aligned}
& +\frac{(\Delta t)^{\alpha}}{2 \Gamma(\alpha+3)}
\end{aligned}
$$

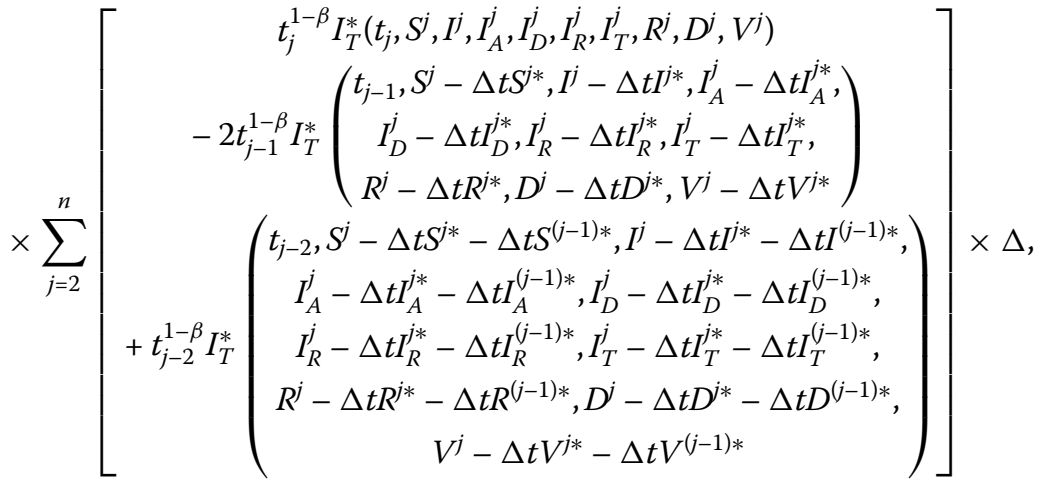

$$
\begin{aligned}
& R^{n+1}=\frac{(\Delta t)^{\alpha}}{\Gamma(\alpha+1)} \sum_{j=2}^{n} t_{j-2}^{1-\beta} R^{*}\left(\begin{array}{c}
t_{j-2}, S^{j}-\Delta t S^{j *}-\Delta t S^{(j-1) *}, I^{j}-\Delta t I^{j *}-\Delta t I^{(j-1) *}, \\
I_{A}^{j}-\Delta t I_{A}^{j *}-\Delta t I_{A}^{(j-1) *}, I_{D}^{j}-\Delta t I_{D}^{j *}-\Delta t I_{D}^{(j-1) *}, \\
I_{R}^{j}-\Delta t I_{R}^{j *}-\Delta t I_{R}^{(j-1) *}, I_{T}^{j}-\Delta t I_{T}^{j *}-\Delta t I_{T}^{(j-1) *}, \\
R^{j}-\Delta t R^{j *}-\Delta t R^{(j-1) *}, D^{j}-\Delta t D^{j *}-\Delta t D^{(j-1) *}, \\
V^{j}-\Delta t V^{j *}-\Delta t V^{(j-1) *}
\end{array}\right) \times \Pi \\
& +\frac{(\Delta t)^{\alpha}}{\Gamma(\alpha+2)}
\end{aligned}
$$

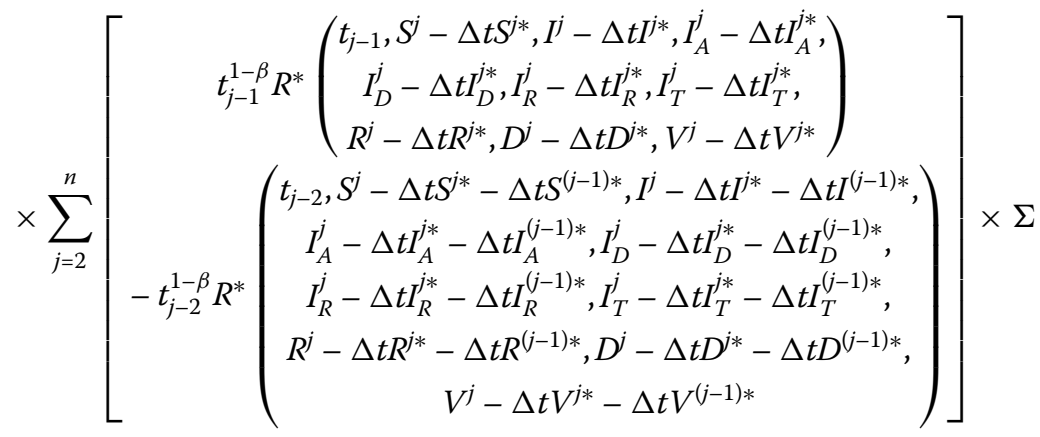

$$
\begin{aligned}
& +\frac{(\Delta t)^{\alpha}}{2 \Gamma(\alpha+3)}
\end{aligned}
$$




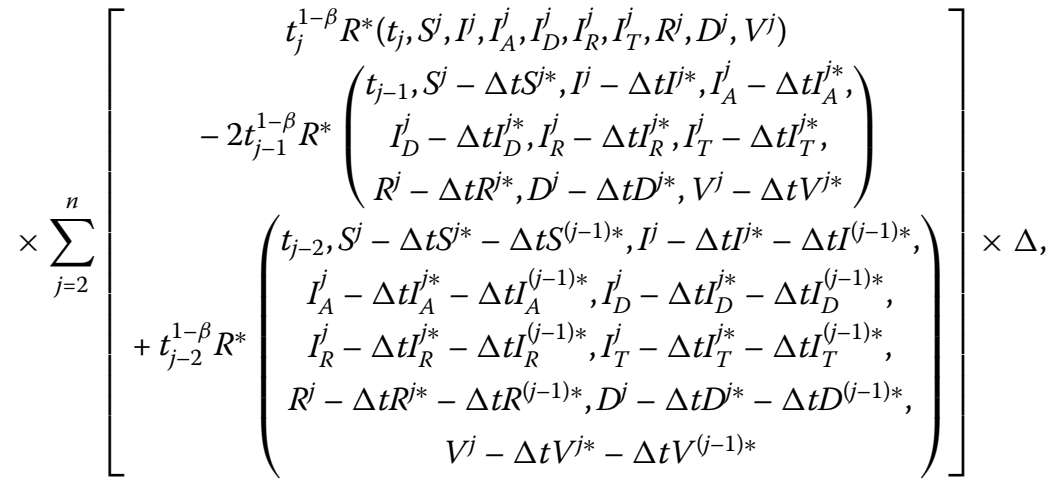

$$
\begin{aligned}
& D^{n+1}=\frac{(\Delta t)^{\alpha}}{\Gamma(\alpha+1)} \sum_{j=2}^{n} t_{j-2}^{1-\beta} D^{*}\left(\begin{array}{c}
t_{j-2}, S^{j}-\Delta t S^{j *}-\Delta t S^{(j-1) *}, I^{j}-\Delta t I^{j *}-\Delta t I^{(j-1) *}, \\
I_{A}^{j}-\Delta t I_{A}^{j *}-\Delta t I_{A}^{(j-1) *}, I_{D}^{j}-\Delta t I_{D}^{j *}-\Delta t I_{D}^{(j-1) *}, \\
I_{R}^{j}-\Delta t I_{R}^{j *}-\Delta t I_{R}^{(j-1) *}, I_{T}^{j}-\Delta t I_{T}^{j *}-\Delta t I_{T}^{(j-1) *}, \\
R^{j}-\Delta t R^{j *}-\Delta t R^{(j-1) *}, D^{j}-\Delta t D^{j *}-\Delta t D^{(j-1) *}, \\
V^{j}-\Delta t V^{j *}-\Delta t V^{(j-1) *}
\end{array}\right) \times \Pi \\
& +\frac{(\Delta t)^{\alpha}}{\Gamma(\alpha+2)} \\
& \times \sum_{j=2}^{n}\left[\begin{array}{c}
t_{j-1}, S^{j}-\Delta t S^{j *}, I^{j}-\Delta t I^{j *}, I_{A}^{j}-\Delta t I_{A}^{j *}, \\
I_{D-1}^{j-\beta}-\Delta t I_{D}^{j *}, I_{R}^{j}-\Delta t I_{R}^{j^{*}}, I_{T}^{j}-\Delta t I_{T}^{j *}, \\
R^{j}-\Delta t R^{j *}, D^{j}-\Delta t D^{j *}, V^{j}-\Delta t V^{j *}
\end{array}\right)\left(\begin{array}{c}
t_{j-2}, S^{j}-\Delta t S^{j *}-\Delta t S^{(j-1) *}, I^{j}-\Delta t I^{j *}-\Delta t I^{(j-1) *}, \\
I_{A}^{j}-\Delta t I_{A}^{j *}-\Delta t I_{A}^{(j-1) *}, I_{D}^{j}-\Delta t I_{D}^{j *}-\Delta t I_{D}^{(j-1) *}, \\
I_{R}^{j}-\Delta t I_{R}^{j *}-\Delta t I_{R}^{(j-1) *}, I_{T}^{j}-\Delta t I_{T}^{j *}-\Delta t I_{T}^{(j-1) *}, \\
R^{j}-\Delta t R^{j *}-\Delta t R^{(j-1) *}, D^{j}-\Delta t D^{j *}-\Delta t D^{(j-1) *}, \\
V^{j}-\Delta t V^{j *}-\Delta t V^{(j-1) *}
\end{array}\right) \times \Sigma \\
& +\frac{(\Delta t)^{\alpha}}{2 \Gamma(\alpha+3)}
\end{aligned}
$$

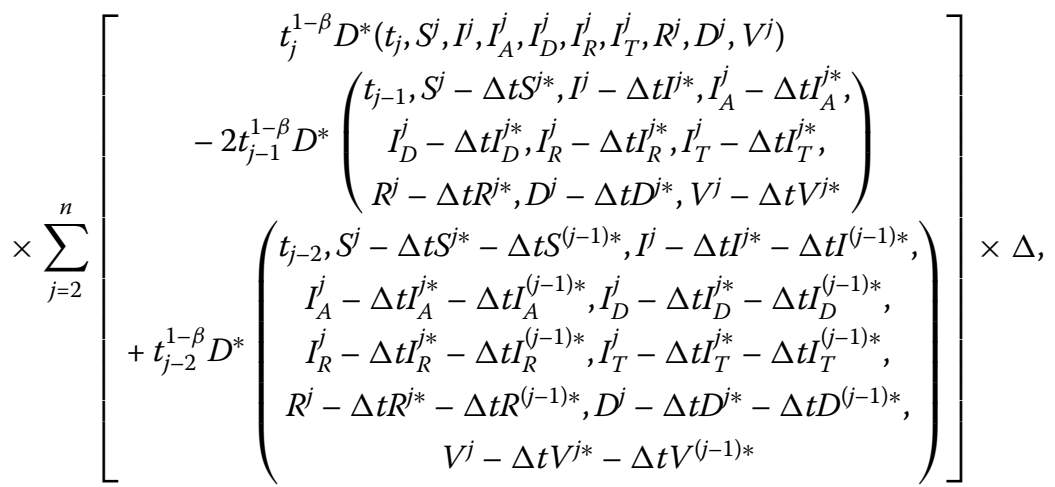

$$
\begin{aligned}
& V^{n+1}=\frac{(\Delta t)^{\alpha}}{\Gamma(\alpha+1)} \sum_{j=2}^{n} t_{j-2}^{1-\beta} V^{*}\left(\begin{array}{c}
t_{j-2}, S^{j}-\Delta t S^{j *}-\Delta t S^{(j-1) *}, I^{j}-\Delta t I^{j *}-\Delta t I^{(j-1) *}, \\
I_{A}^{j}-\Delta t I_{A}^{j *}-\Delta t I_{A}^{(j-1) *}, I_{D}^{j}-\Delta t I_{D}^{j *}-\Delta t I_{D}^{(j-1) *}, \\
I_{R}^{j}-\Delta t I_{R}^{j *}-\Delta t I_{R}^{(j-1) *}, I_{T}^{j}-\Delta t I_{T}^{j *}-\Delta t I_{T}^{(j-1) *}, \\
R^{j}-\Delta t R^{j *}-\Delta t R^{(j-1) *}, D^{j}-\Delta t D^{j *}-\Delta t D^{(j-1) *}, \\
V^{j}-\Delta t V^{j *}-\Delta t V^{(j-1) *}
\end{array}\right) \times \Pi
\end{aligned}
$$




$$
\begin{aligned}
& +\frac{(\Delta t)^{\alpha}}{\Gamma(\alpha+2)}
\end{aligned}
$$

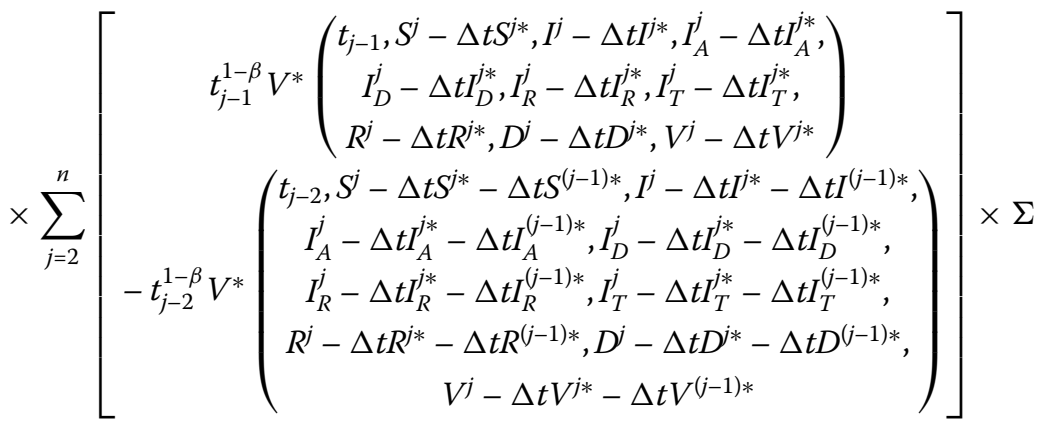

$$
\begin{aligned}
& +\frac{(\Delta t)^{\alpha}}{2 \Gamma(\alpha+3)}
\end{aligned}
$$

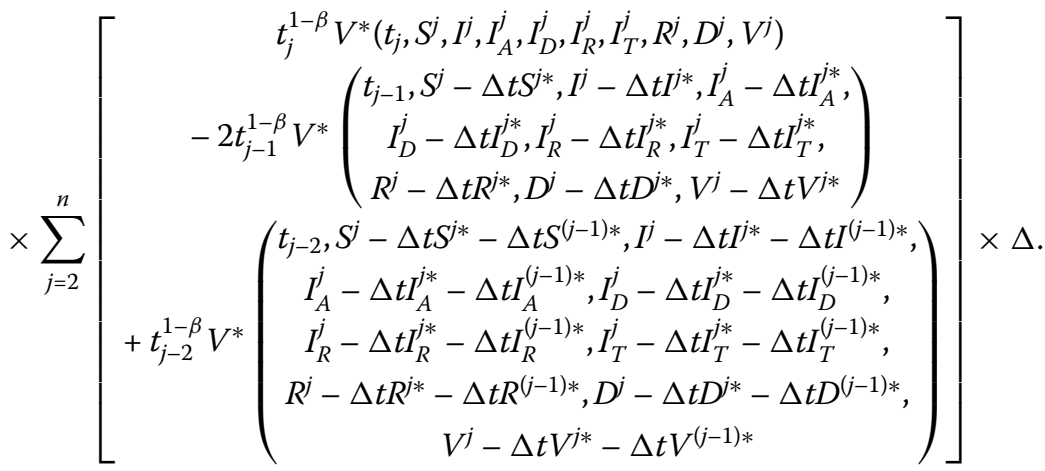

Now we apply

$$
\begin{aligned}
& { }_{0}^{F F E} D_{t}^{\alpha, \beta(t)} S=S^{*}\left(t, S, I, I_{A}, I_{D}, I_{R}, I_{T}, R, D, V\right), \\
& { }_{0}^{F F E} D_{t}^{\alpha, \beta(t)} I=I^{*}\left(t, S, I, I_{A}, I_{D}, I_{R}, I_{T}, R, D, V\right), \\
& { }_{0}^{F F E} D_{t}^{\alpha, \beta(t)} I_{A}=I_{A}^{*}\left(t, S, I, I_{A}, I_{D}, I_{R}, I_{T}, R, D, V\right), \\
& { }_{0}^{F F E} D_{t}^{\alpha, \beta(t)} I_{D}=I_{D}^{*}\left(t, S, I, I_{A}, I_{D}, I_{R}, I_{T}, R, D, V\right), \\
& { }_{0}^{F F E} D_{t}^{\alpha, \beta(t)} I_{R}=I_{R}^{*}\left(t, S, I, I_{A}, I_{D}, I_{R}, I_{T}, R, D, V\right), \\
& { }_{0}^{F F E} D_{t}^{\alpha, \beta(t)} I_{T}=I_{T}^{*}\left(t, S, I, I_{A}, I_{D}, I_{R}, I_{T}, R, D, V\right), \\
& { }_{0}^{F F E} D_{t}^{\alpha, \beta(t)} R=R^{*}\left(t, S, I, I_{A}, I_{D}, I_{R}, I_{T}, R, D, V\right), \\
& { }_{0}^{F F E} D_{t}^{\alpha, \beta(t)} D=D^{*}\left(t, S, I, I_{A}, I_{D}, I_{R}, I_{T}, R, D, V\right), \\
& { }_{0}^{F F E} D_{t}^{\alpha, \beta(t)} V=V^{*}\left(t, S, I, I_{A}, I_{D}, I_{R}, I_{T}, R, D, V\right) .
\end{aligned}
$$

After applying the fractional integral with exponential kernel and putting the Newton polynomial into these equations, we can solve our model as follows:

$$
S^{n+1}=S^{n}+\frac{1-\alpha}{M(\alpha)}\left[\begin{array}{c}
t_{n+1}^{2-\beta\left(t_{n+1}\right)}\left(-\frac{\beta\left(t_{n+2}\right)-\beta\left(t_{n+1}\right)}{\Delta t} \ln t_{n+1}+\frac{2-\beta\left(t_{n+1}\right)}{t_{n+1}}\right) \\
\left(\begin{array}{c}
t_{n+1}, S^{n}+\Delta t S^{n *}, I^{n}+\Delta t I^{n *}, I_{A}^{n}+\Delta t I_{A}^{n *}, \\
I_{D}^{n}+\Delta t I_{D}^{n *}, I_{R}^{n}+\Delta t I_{R}^{n *}, I_{T}^{n}+\Delta t I_{T}^{n *}, \\
R^{n}+\Delta t R^{n *}, D^{n}+\Delta t D^{n *}, V^{n}+\Delta t V^{n *}
\end{array}\right) \\
-t_{n}^{2-\beta\left(t_{n}\right)}\left(-\frac{\beta\left(t_{n+1}\right)-\beta\left(t_{n}\right)}{\Delta t} \ln t_{n}+\frac{2-\beta\left(t_{n}\right)}{t_{n}}\right) \\
S^{*}\left(t_{n}, S^{n}, I^{n}, I_{A}^{n}, I_{D}^{n}, I_{R}^{n}, I_{T}^{n}, R^{n}, D^{n}, V^{n}\right)
\end{array}\right]
$$




$$
\left.+\frac{\alpha}{M(\alpha)}\left\{\begin{array}{c}
\frac{23}{12} t_{n}^{2-\beta\left(t_{n}\right)}\left(-\frac{\beta\left(t_{n+1}\right)-\beta\left(t_{n}\right)}{\Delta t} \ln t_{n}+\frac{2-\beta\left(t_{n}\right)}{t_{n}}\right) \\
\times S^{*}\left(t_{n}, S^{n}, I^{n}, I_{A}^{n}, I_{D}^{n}, I_{R}^{n}, I_{T}^{n}, R^{n}, D^{n}, V^{n}\right) \Delta t \\
-\frac{4}{3} t_{n-1}^{2-\beta\left(t_{n-1}\right)}\left(-\frac{\beta\left(t_{n}\right)-\beta\left(t_{n-1}\right)}{\Delta t} \ln t_{n-1}+\frac{2-\beta\left(t_{n-1}\right)}{t_{n-1}}\right) \\
\times S^{*}\left(\begin{array}{c}
t_{n-1}, S^{n}-\Delta t S^{n *}, I^{n}-\Delta t I^{n *}, I_{A}^{n}-\Delta t I_{A}^{n *}, \\
I_{D}^{n}-\Delta t I_{D}^{n *}, I_{R}^{n}-\Delta t I_{R}^{n *}, I_{T}^{n}-\Delta t I_{T}^{n *}, \\
R^{n}-\Delta t R^{n *}, D^{n}-\Delta t D^{n *}, V^{n}-\Delta t V^{n *}
\end{array}\right) \Delta t \\
+\frac{5}{12} t_{n-2}^{2-\beta\left(t_{n-2}\right)}\left(-\frac{\beta\left(t_{n-1}\right)-\beta\left(t_{n-2}\right)}{\Delta t} \ln t_{n-2}+\frac{2-\beta\left(t_{n-2}\right)}{t_{n-2}}\right) \\
t_{n-2}, S^{n}-\Delta t S^{n *}-\Delta t S^{(n-1) *}, I^{n}-\Delta t I^{n *}-\Delta t I^{(n-1) *}, \\
I_{A}^{n}-\Delta t I_{A}^{n *}-\Delta t I_{A}^{(n-1) *}, I_{D}^{n}-\Delta t I_{D}^{n *}-\Delta t I_{D}^{(n-1) *}, \\
I_{R}^{n}-\Delta t I_{R}^{n *}-\Delta t I_{R}^{(n-1) *}, I_{T}^{n}-\Delta t I_{T}^{n *}-\Delta t I_{T}^{(n-1) *}, \\
R^{n}-\Delta t R^{n *}-\Delta t R^{(n-1) *}, D^{n}-\Delta t D^{n *}-\Delta t D^{(n-1) *}, \\
V^{n}-\Delta t V^{n *}-\Delta t V^{(n-1) *}
\end{array}\right) \Delta t\right\},
$$

$$
I^{n+1}=I^{n}+\frac{1-\alpha}{M(\alpha)}
$$

$$
\begin{aligned}
& \times\left[\begin{array}{c}
t_{n+1}^{2-\beta\left(t_{n+1}\right)}\left(-\frac{\beta\left(t_{n+2}\right)-\beta\left(t_{n+1}\right)}{\Delta t} \ln t_{n+1}+\frac{2-\beta\left(t_{n+1}\right)}{t_{n+1}}\right) \\
\times I^{*}\left(\begin{array}{c}
t_{n+1}, S^{n}+\Delta t S^{n *}, I^{n}+\Delta t I^{n *}, I_{A}^{n}+\Delta t I_{A}^{n *}, I_{D}^{n}+\Delta t I_{D}^{n *}, \\
I_{R}^{n}+\Delta t I_{R}^{n *}, I_{T}^{n}+\Delta t I_{T}^{n *}, R^{n}+\Delta t R^{n *}, D^{n}+\Delta t D^{n *}, V^{n}+\Delta t V^{n *}
\end{array}\right) \\
-t_{n}^{2-\beta\left(t_{n}\right)}\left(-\frac{\beta\left(t_{n+1}\right)-\beta\left(t_{n}\right)}{\Delta t} \ln t_{n}+\frac{2-\beta\left(t_{n}\right)}{t_{n}}\right) \\
I^{*}\left(t_{n}, S^{n}, I^{n}, I_{A}^{n}, I_{D}^{n}, I_{R}^{n}, I_{T}^{n}, R^{n}, D^{n}, V^{n}\right)
\end{array}\right] \\
& \left.+\frac{\alpha}{M(\alpha)}\left\{\begin{array}{c}
\frac{23}{12} t_{n}^{2-\beta\left(t_{n}\right)}\left(-\frac{\beta\left(t_{n+1}\right)-\beta\left(t_{n}\right)}{\Delta t} \ln t_{n}+\frac{2-\beta\left(t_{n}\right)}{t_{n}}\right) \\
\times I^{*}\left(t_{n}, S^{n}, I^{n}, I_{A}^{n}, I_{D}^{n}, I_{R}^{n}, I_{T}^{n}, R^{n}, D^{n}, V^{n}\right) \Delta t \\
-\frac{4}{3} t_{n-1}^{2-\beta\left(t_{n-1}\right)}\left(-\frac{\beta\left(t_{n}\right)-\beta\left(t_{n-1}\right)}{\Delta t} \ln t_{n-1}+\frac{2-\beta\left(t_{n-1}\right)}{t_{n-1}}\right) \\
\times I^{*}\left(\begin{array}{c}
t_{n-1}, S^{n}-\Delta t S^{n *}, I^{n}-\Delta t I^{n *}, I_{A}^{n}-\Delta t I_{A}^{n *}, \\
I_{D}^{n}-\Delta t I_{D}^{n *}, I_{R}^{n}-\Delta t I_{R}^{n *}, I_{T}^{n}-\Delta t I_{T}^{n *}, \\
R^{n}-\Delta t R^{n *}, D^{n}-\Delta t D^{n *}, V^{n}-\Delta t V^{n *}
\end{array}\right) \Delta t \\
+\frac{5}{12} t_{n-2}^{2-\beta\left(t_{n-2}\right)}\left(-\frac{\beta\left(t_{n-1}\right)-\beta\left(t_{n-2}\right)}{\Delta t} \ln t_{n-2}+\frac{2-\beta\left(t_{n-2}\right)}{t_{n-2}}\right) \\
t_{n-2}, S^{n}-\Delta t S^{n *}-\Delta t S^{(n-1) *}, I^{n}-\Delta t I^{n *}-\Delta t I^{(n-1) *}, \\
I_{A}^{n}-\Delta t I_{A}^{n *}-\Delta t I_{A}^{(n-1) *}, I_{D}^{n}-\Delta t I_{D}^{n *}-\Delta t I_{D}^{(n-1) *}, \\
I_{R}^{n}-\Delta t I_{R}^{n *}-\Delta t I_{R}^{(n-1) *}, I_{T}^{n}-\Delta t I_{T}^{n *}-\Delta t I_{T}^{(n-1) *}, \\
R^{n}-\Delta t R^{n *}-\Delta t R^{(n-1) *}, D^{n}-\Delta t D^{n *}-\Delta t D^{(n-1) *}, \\
V^{n}-\Delta t V^{n *}-\Delta t V^{(n-1) *}
\end{array}\right\}, \Delta t\right\}, \\
& I_{A}^{n+1}=I_{A}^{n}+\frac{1-\alpha}{M(\alpha)}\left[\begin{array}{c}
t_{n+1}^{2-\beta\left(t_{n+1}\right)}\left(-\frac{\beta\left(t_{n+2}\right)-\beta\left(t_{n+1}\right)}{\Delta t} \ln t_{n+1}+\frac{2-\beta\left(t_{n+1}\right)}{t_{n+1}}\right) \\
\left(\begin{array}{c}
t_{n+1}, S^{n}+\Delta t S^{n *}, I^{n}+\Delta t I^{n *}, I_{A}^{n}+\Delta t I_{A}^{n *}, \\
I_{D}^{n}+\Delta t I_{D}^{n *}, I_{R}^{n}+\Delta t I_{R}^{n *}, I_{T}^{n}+\Delta t I_{T}^{n *}, \\
R^{n}+\Delta t R^{n *}, D^{n}+\Delta t D^{n *}, V^{n}+\Delta t V^{n *}
\end{array}\right) \\
-t_{n}^{2-\beta\left(t_{n}\right)}\left(-\frac{\beta\left(t_{n+1}\right)-\beta\left(t_{n}\right)}{\Delta t} \ln t_{n}+\frac{2-\beta\left(t_{n}\right)}{t_{n}}\right) \\
\times I_{A}^{*}\left(t_{n}, S^{n}, I^{n}, I_{A}^{n}, I_{D}^{n}, I_{R}^{n}, I_{T}^{n}, R^{n}, D^{n}, V^{n}\right)
\end{array}\right]
\end{aligned}
$$




$$
\begin{aligned}
& \left.+\frac{\alpha}{M(\alpha)}\left\{\begin{array}{c}
\frac{23}{12} t_{n}^{2-\beta\left(t_{n}\right)}\left(-\frac{\beta\left(t_{n+1}\right)-\beta\left(t_{n}\right)}{\Delta t} \ln t_{n}+\frac{2-\beta\left(t_{n}\right)}{t_{n}}\right) \\
\times I_{A}^{*}\left(t_{n}, S^{n}, I^{n}, I_{A}^{n}, I_{D}^{n}, I_{R}^{n}, I_{T}^{n}, R^{n}, D^{n}, V^{n}\right) \Delta t \\
-\frac{4}{3} t_{n-1}^{2-\beta\left(t_{n-1}\right)}\left(-\frac{\beta\left(t_{n}\right)-\beta\left(t_{n-1}\right)}{\Delta t} \ln t_{n-1}+\frac{2-\beta\left(t_{n-1}\right)}{t_{n-1}}\right) \\
\times\left(\begin{array}{c}
t_{n-1}, S^{n}-\Delta t S^{n *}, I^{n}-\Delta t I^{n *}, I_{A}^{n}-\Delta t I_{A}^{n *}, \\
I_{D}^{n}-\Delta t I_{D}^{n *}, I_{R}^{n}-\Delta t I_{R}^{n *}, I_{T}^{n}-\Delta t I_{T}^{n *}, \\
R^{n}-\Delta t R^{n *}, D^{n}-\Delta t D^{n *}, V^{n}-\Delta t V^{n *}
\end{array}\right) \Delta t \\
+\frac{5}{12} t_{n-2}^{2-\beta\left(t_{n-2}\right)}\left(-\frac{\beta\left(t_{n-1}\right)-\beta\left(t_{n-2}\right)}{\Delta t} \ln t_{n-2}+\frac{2-\beta\left(t_{n-2}\right)}{t_{n-2}}\right) \\
t_{n-2}, S^{n}-\Delta t S^{n *}-\Delta t S^{(n-1) *}, I^{n}-\Delta t I^{n *}-\Delta t I^{(n-1) *}, \\
I_{A}^{n}-\Delta t I_{A}^{n *}-\Delta t I_{A}^{(n-1) *}, I_{D}^{n}-\Delta t I_{D}^{n *}-\Delta t I_{D}^{(n-1) *}, \\
I_{R}^{n}-\Delta t I_{R}^{n *}-\Delta t I_{R}^{(n-1) *}, I_{T}^{n}-\Delta t I_{T}^{n *}-\Delta t I_{T}^{(n-1) *}, \\
R^{n}-\Delta t R^{n *}-\Delta t R^{(n-1) *}, D^{n}-\Delta t D^{n *}-\Delta t D^{(n-1) *}, \\
V^{n}-\Delta t V^{n *}-\Delta t V^{(n-1) *}
\end{array}\right\}, \Delta t\right\}, \\
& I_{D}^{n+1}=I_{D}^{n}+\frac{1-\alpha}{M(\alpha)} \\
& \times\left[\begin{array}{c}
t_{n+1}^{2-\beta\left(t_{n+1}\right)}\left(-\frac{\beta\left(t_{n+2}\right)-\beta\left(t_{n+1}\right)}{\Delta t} \ln t_{n+1}+\frac{2-\beta\left(t_{n+1}\right)}{t_{n+1}}\right) \\
\times I_{D}^{*}\left(\begin{array}{c}
t_{n+1}, S^{n}+\Delta t S^{n *}, I^{n}+\Delta t I^{n *}, I_{A}^{n}+\Delta t I_{A}^{n *}, I_{D}^{n}+\Delta t I_{D}^{n *}, \\
I_{R}^{n}+\Delta t I_{R}^{n *}, I_{T}^{n}+\Delta t I_{T}^{n *}, R^{n}+\Delta t R^{n *}, D^{n}+\Delta t D^{n *}, V^{n}+\Delta t V^{n *}
\end{array}\right) \\
-t_{n}^{2-\beta\left(t_{n}\right)}\left(-\frac{\beta\left(t_{n+1}\right)-\beta\left(t_{n}\right)}{\Delta t} \ln t_{n}+\frac{2-\beta\left(t_{n}\right)}{t_{n}}\right) \\
\times I_{D}^{*}\left(t_{n}, S^{n}, I^{n}, I_{A}^{n}, I_{D}^{n}, I_{R}^{n}, I_{T}^{n}, R^{n}, D^{n}, V^{n}\right)
\end{array}\right] \\
& +\frac{\alpha}{M(\alpha)}\left\{\begin{array}{c}
\frac{23}{12} t_{n}^{2-\beta\left(t_{n}\right)}\left(-\frac{\beta\left(t_{n+1}\right)-\beta\left(t_{n}\right)}{\Delta t} \ln t_{n}+\frac{2-\beta\left(t_{n}\right)}{t_{n}}\right) \\
\times I_{D}^{*}\left(t_{n}, S^{n}, I^{n}, I_{A}^{n}, I_{D}^{n}, I_{R}^{n}, I_{T}^{n}, R^{n}, D^{n}, V^{n}\right) \Delta t \\
-\frac{4}{3} t_{n-1}^{2-\beta\left(t_{n-1}\right)}\left(-\frac{\beta\left(t_{n}\right)-\beta\left(t_{n-1}\right)}{\Delta t} \ln t_{n-1}+\frac{2-\beta\left(t_{n-1}\right)}{t_{n-1}}\right) \\
\times I_{D}^{*}\left(\begin{array}{c}
t_{n-1}, S^{n}-\Delta t S^{n *}, I^{n}-\Delta t I^{n *}, I_{A}^{n}-\Delta t I_{A}^{n *}, \\
I_{D}^{n}-\Delta t I_{D}^{n *}, I_{R}^{n}-\Delta t I_{R}^{n *}, I_{T}^{n}-\Delta t I_{T}^{n *}, \\
R^{n}-\Delta t R^{n *}, D^{n}-\Delta t D^{n *}, V^{n}-\Delta t V^{n *}
\end{array}\right) \Delta t \\
+\frac{5}{12} t_{n-2}^{2-\beta\left(t_{n-2}\right)}\left(-\frac{\beta\left(t_{n-1}\right)-\beta\left(t_{n-2}\right)}{\Delta t} \ln t_{n-2}+\frac{2-\beta\left(t_{n-2}\right)}{t_{n-2}}\right) \\
t_{n-2}, S^{n}-\Delta t S^{n *}-\Delta t S^{(n-1) *}, I^{n}-\Delta t I^{n *}-\Delta t I^{(n-1) *}, \\
I_{A}^{n}-\Delta t I_{A}^{n *}-\Delta t I_{A}^{(n-1) *}, I_{D}^{n}-\Delta t I_{D}^{n *}-\Delta t I_{D}^{(n-1) *}, \\
I_{R}^{n}-\Delta t I_{R}^{n *}-\Delta t I_{R}^{(n-1) *}, I_{T}^{n}-\Delta t I_{T}^{n *}-\Delta t I_{T}^{(n-1) *}, \\
R^{n}-\Delta t R^{n *}-\Delta t R^{(n-1) *}, D^{n}-\Delta t D^{n *}-\Delta t D^{(n-1) *}, \\
V^{n}-\Delta t V^{n *}-\Delta t V^{(n-1) *}
\end{array}\right\} \Delta t
\end{aligned}
$$

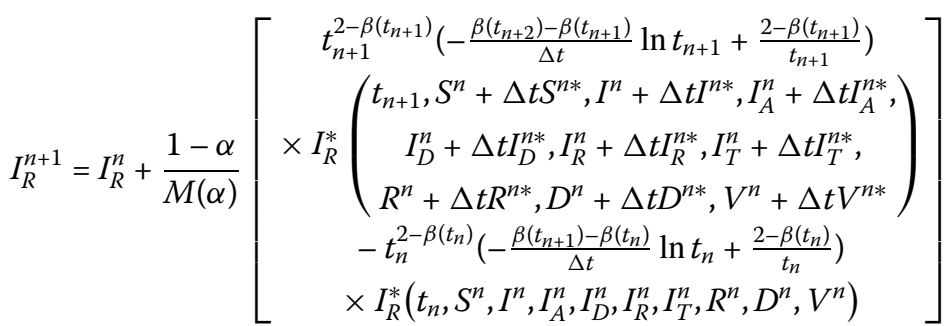




$$
\begin{aligned}
& \left.+\frac{\alpha}{M(\alpha)}\left\{\begin{array}{c}
\frac{23}{12} t_{n}^{2-\beta\left(t_{n}\right)}\left(-\frac{\beta\left(t_{n+1}\right)-\beta\left(t_{n}\right)}{\Delta t} \ln t_{n}+\frac{2-\beta\left(t_{n}\right)}{t_{n}}\right) \\
\times I_{D}^{*}\left(t_{n}, S^{n}, I^{n}, I_{A}^{n}, I_{D}^{n}, I_{R}^{n}, I_{T}^{n}, R^{n}, D^{n}, V^{n}\right) \Delta t \\
-\frac{4}{3} t_{n-1}^{2-\beta\left(t_{n-1}\right)}\left(-\frac{\beta\left(t_{n}\right)-\beta\left(t_{n-1}\right)}{\Delta t} \ln t_{n-1}+\frac{2-\beta\left(t_{n-1}\right)}{t_{n-1}}\right) \\
\times I_{R}^{*}\left(\begin{array}{c}
t_{n-1}, S^{n}-\Delta t S^{n *}, I^{n}-\Delta t I^{n *}, I_{A}^{n}-\Delta t I_{A}^{n *}, \\
I_{D}^{n}-\Delta t I_{D}^{n *}, I_{R}^{n}-\Delta t I_{R}^{n *}, I_{T}^{n}-\Delta t I_{T}^{n *}, \\
R^{n}-\Delta t R^{n *}, D^{n}-\Delta t D^{n *}, V^{n}-\Delta t V^{n *}
\end{array}\right) \Delta t \\
+\frac{5}{12} t_{n-2}^{2-\beta\left(t_{n-2}\right)}\left(-\frac{\beta\left(t_{n-1}\right)-\beta\left(t_{n-2}\right)}{\Delta t} \ln t_{n-2}+\frac{2-\beta\left(t_{n-2}\right)}{t_{n-2}}\right) \\
t_{n-2}, S^{n}-\Delta t S^{n *}-\Delta t S^{(n-1) *}, I^{n}-\Delta t I^{n *}-\Delta t I^{(n-1) *}, \\
I_{A}^{n}-\Delta t I_{A}^{n *}-\Delta t I_{A}^{(n-1) *}, I_{D}^{n}-\Delta t I_{D}^{n *}-\Delta t I_{D}^{(n-1) *}, \\
I_{R}^{n}-\Delta t I_{R}^{n *}-\Delta t I_{R}^{(n-1) *}, I_{T}^{n}-\Delta t I_{T}^{n *}-\Delta t I_{T}^{(n-1) *}, \\
R^{n}-\Delta t R^{n *}-\Delta t R^{(n-1) *}, D^{n}-\Delta t D^{n *}-\Delta t D^{(n-1) *}, \\
V^{n}-\Delta t V^{n *}-\Delta t V^{(n-1) *}
\end{array}\right\} \Delta t\right\} \\
& I_{T}^{n+1}=I_{T}^{n}+\frac{1-\alpha}{M(\alpha)} \\
& \left.\times\left[\begin{array}{c}
t_{n+1}^{2-\beta\left(t_{n+1}\right)}\left(-\frac{\beta\left(t_{n+2}\right)-\beta\left(t_{n+1}\right)}{\Delta t} \ln t_{n+1}+\frac{2-\beta\left(t_{n+1}\right)}{t_{n+1}}\right) \\
t_{n+1}, S^{n}+\Delta t S^{n *}, I^{n}+\Delta t I^{n *}, I_{A}^{n}+\Delta t I_{A}^{n *}, I_{D}^{n}+\Delta t I_{D}^{n *}, \\
I_{R}^{n}+\Delta t I_{R}^{n *}, I_{T}^{n}+\Delta t I_{T}^{n *}, R^{n}+\Delta t R^{n *}, D^{n}+\Delta t D^{n *}, V^{n}+\Delta t V^{n *}
\end{array}\right)\right] \\
& \left.+\frac{\alpha}{M(\alpha)}\left\{\begin{array}{c}
\frac{23}{12} t_{n}^{2-\beta\left(t_{n}\right)}\left(-\frac{\beta\left(t_{n+1}\right)-\beta\left(t_{n}\right)}{\Delta t} \ln t_{n}+\frac{2-\beta\left(t_{n}\right)}{t_{n}}\right) \\
\times I_{T}^{*}\left(t_{n}, S^{n}, I^{n}, I_{A}^{n}, I_{D}^{n}, I_{R}^{n}, I_{T}^{n}, R^{n}, D^{n}, V^{n}\right) \Delta t \\
-\frac{4}{3} t_{n-1}^{2-\beta\left(t_{n-1}\right)}\left(-\frac{\beta\left(t_{n}\right)-\beta\left(t_{n-1}\right)}{\Delta t} \ln t_{n-1}+\frac{2-\beta\left(t_{n-1}\right)}{t_{n-1}}\right) \\
\times I_{T}^{*}\left(\begin{array}{c}
t_{n-1}, S^{n}-\Delta t S^{n *}, I^{n}-\Delta t I^{n *}, I_{A}^{n}-\Delta t I_{A}^{n *}, \\
I_{D}^{n}-\Delta t I_{D}^{n *}, I_{R}^{n}-\Delta t I_{R}^{n *}, I_{T}^{n}-\Delta t I_{T}^{n *}, \\
R^{n}-\Delta t R^{n *}, D^{n}-\Delta t D^{n *}, V^{n}-\Delta t V^{n *}
\end{array}\right) \Delta t \\
+\frac{5}{12} t_{n-2}^{2-\beta\left(t_{n-2}\right)}\left(-\frac{\beta\left(t_{n-1}\right)-\beta\left(t_{n-2}\right)}{\Delta t} \ln t_{n-2}+\frac{2-\beta\left(t_{n-2}\right)}{t_{n-2}}\right) \\
t_{n-2}, S^{n}-\Delta t S^{n *}-\Delta t S^{(n-1) *}, I^{n}-\Delta t I^{n *}-\Delta t I^{(n-1) *}, \\
I_{A}^{n}-\Delta t I_{A}^{n *}-\Delta t I_{A}^{(n-1) *}, I_{D}^{n}-\Delta t I_{D}^{n *}-\Delta t I_{D}^{(n-1) *}, \\
I_{R}^{n}-\Delta t I_{R}^{n *}-\Delta t I_{R}^{(n-1) *}, I_{T}^{n}-\Delta t I_{T}^{n *}-\Delta t I_{T}^{(n-1) *}, \\
R^{n}-\Delta t R^{n *}-\Delta t R^{(n-1) *}, D^{n}-\Delta t D^{n *}-\Delta t D^{(n-1) *}, \\
V^{n}-\Delta t V^{n *}-\Delta t V^{(n-1) *}
\end{array}\right\}, \Delta t\right\}, \\
& R^{n+1}=R^{n}+\frac{1-\alpha}{M(\alpha)}
\end{aligned}
$$

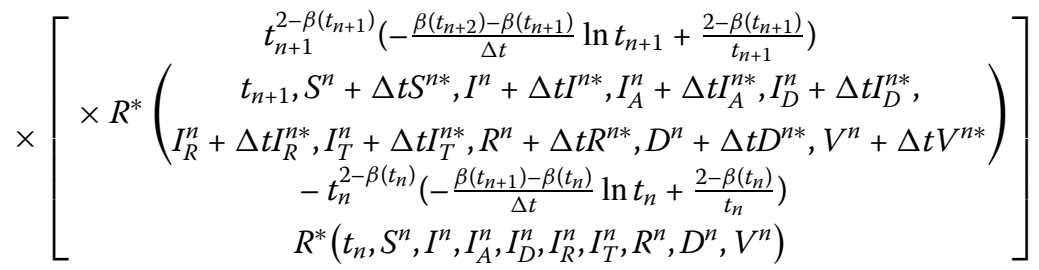




$$
+\frac{\alpha}{M(\alpha)}\left\{\begin{array}{c}
\frac{23}{12} t_{n}^{2-\beta\left(t_{n}\right)}\left(-\frac{\beta\left(t_{n+1}\right)-\beta\left(t_{n}\right)}{\Delta t} \ln t_{n}+\frac{2-\beta\left(t_{n}\right)}{t_{n}}\right) \\
\times R^{*}\left(t_{n}, S^{n}, I^{n}, I_{A}^{n}, I_{D}^{n}, I_{R}^{n}, I_{T}^{n}, R^{n}, D^{n}, V^{n}\right) \Delta t \\
-\frac{4}{3} t_{n-1}^{2-\beta\left(t_{n-1}\right)}\left(-\frac{\beta\left(t_{n}\right)-\beta\left(t_{n-1}\right)}{\Delta t} \ln t_{n-1}+\frac{2-\beta\left(t_{n-1}\right)}{t_{n-1}}\right) \\
\times R^{*}\left(\begin{array}{c}
t_{n-1}, S^{n}-\Delta t S^{n *}, I^{n}-\Delta t I^{n *}, I_{A}^{n}-\Delta t I_{A}^{n *}, \\
I_{D}^{n}-\Delta t I_{D}^{n *}, I_{R}^{n}-\Delta t I_{R}^{n *}, I_{T}^{n}-\Delta t I_{T}^{n *}, \\
R^{n}-\Delta t R^{n *}, D^{n}-\Delta t D^{n *}, V^{n}-\Delta t V^{n *}
\end{array}\right) \Delta t \\
+\frac{5}{12} t_{n-2}^{2-\beta\left(t_{n-2}\right)}\left(-\frac{\beta\left(t_{n-1}\right)-\beta\left(t_{n-2}\right)}{\Delta t} \ln t_{n-2}+\frac{2-\beta\left(t_{n-2}\right)}{t_{n-2}}\right) \\
t_{n-2}, S^{n}-\Delta t S^{n *}-\Delta t S^{(n-1) *}, I^{n}-\Delta t I^{n *}-\Delta t I^{(n-1) *}, \\
I_{A}^{n}-\Delta t I_{A}^{n *}-\Delta t I_{A}^{(n-1) *}, I_{D}^{n}-\Delta t I_{D}^{n *}-\Delta t I_{D}^{(n-1) *}, \\
I_{R}^{n}-\Delta t I_{R}^{n *}-\Delta t I_{R}^{(n-1) *}, I_{T}^{n}-\Delta t I_{T}^{n *}-\Delta t I_{T}^{(n-1) *}, \\
R^{n}-\Delta t R^{n *}-\Delta t R^{(n-1) *}, D^{n}-\Delta t D^{n *}-\Delta t D^{(n-1) *}, \\
V^{n}-\Delta t V^{n *}-\Delta t V^{(n-1) *}
\end{array}\right) \Delta t
$$

$$
D^{n+1}=D^{n}+\frac{1-\alpha}{M(\alpha)}
$$$$
\left.\times\left[\begin{array}{c}
t_{n+1}^{2-\beta\left(t_{n+1}\right)}\left(-\frac{\beta\left(t_{n+2}\right)-\beta\left(t_{n+1}\right)}{\Delta t} \ln t_{n+1}+\frac{2-\beta\left(t_{n+1}\right)}{t_{n+1}}\right) \\
t_{n+1}, S^{n}+\Delta t S^{n *}, I^{n}+\Delta t I^{n *}, I_{A}^{n}+\Delta t I_{A}^{n *}, I_{D}^{n}+\Delta t I_{D}^{n *}, \\
I_{R}^{n}+\Delta t I_{R}^{n *}, I_{T}^{n}+\Delta t I_{T}^{n *}, R^{n}+\Delta t R^{n *}, D^{n}+\Delta t D^{n *}, V^{n}+\Delta t V^{n *}
\end{array}\right)\right]
$$

$$
\left.+\frac{\alpha}{M(\alpha)}\left\{\begin{array}{c}
\frac{23}{12} t_{n}^{2-\beta\left(t_{n}\right)}\left(-\frac{\beta\left(t_{n+1}\right)-\beta\left(t_{n}\right)}{\Delta t} \ln t_{n}+\frac{2-\beta\left(t_{n}\right)}{t_{n}}\right) \\
\times D^{*}\left(t_{n}, S^{n}, I^{n}, I_{A}^{n}, I_{D}^{n}, I_{R}^{n}, I_{T}^{n}, R^{n}, D^{n}, V^{n}\right) \Delta t \\
-\frac{4}{3} t_{n-1}^{2-\beta\left(t_{n-1}\right)}\left(-\frac{\beta\left(t_{n}\right)-\beta\left(t_{n-1}\right)}{\Delta t} \ln t_{n-1}+\frac{2-\beta\left(t_{n-1}\right)}{t_{n-1}}\right) \\
\times D^{*}\left(\begin{array}{c}
t_{n-1}, S^{n}-\Delta t S^{n *}, I^{n}-\Delta t I^{n *}, I_{A}^{n}-\Delta t I_{A}^{n *}, \\
I_{D}^{n}-\Delta t I_{D}^{n *}, I_{R}^{n}-\Delta t I_{R}^{n *}, I_{T}^{n}-\Delta t I_{T}^{n *}, \\
R^{n}-\Delta t R^{n *}, D^{n}-\Delta t D^{n *}, V^{n}-\Delta t V^{n *}
\end{array}\right) \Delta t \\
+\frac{5}{12} t_{n-2}^{2-\beta\left(t_{n-2}\right)}\left(-\frac{\beta\left(t_{n-1}\right)-\beta\left(t_{n-2}\right)}{\Delta t} \ln t_{n-2}+\frac{2-\beta\left(t_{n-2}\right)}{t_{n-2}}\right) \\
t_{n-2}, S^{n}-\Delta t S^{n *}-\Delta t S^{(n-1) *}, I^{n}-\Delta t I^{n *}-\Delta t I^{(n-1) *}, \\
I_{A}^{n}-\Delta t I_{A}^{n *}-\Delta t I_{A}^{(n-1) *}, I_{D}^{n}-\Delta t I_{D}^{n *}-\Delta t I_{D}^{(n-1) *}, \\
I_{R}^{n}-\Delta t I_{R}^{n *}-\Delta t I_{R}^{(n-1) *}, I_{T}^{n}-\Delta t I_{T}^{n *}-\Delta t I_{T}^{(n-1) *}, \\
R^{n}-\Delta t R^{n *}-\Delta t R^{(n-1) *}, D^{n}-\Delta t D^{n *}-\Delta t D^{(n-1) *}, \\
V^{n}-\Delta t V^{n *}-\Delta t V^{(n-1) *}
\end{array}\right) \Delta t\right\},
$$

$$
V^{n+1}=V^{n}+\frac{1-\alpha}{M(\alpha)}
$$

$$
\times\left[\begin{array}{c}
t_{n+1}^{2-\beta\left(t_{n+1}\right)}\left(-\frac{\beta\left(t_{n+2}\right)-\beta\left(t_{n+1}\right)}{\Delta t} \ln t_{n+1}+\frac{2-\beta\left(t_{n+1}\right)}{t_{n+1}}\right) \\
\times V^{*}\left(\begin{array}{c}
t_{n+1}, S^{n}+\Delta t S^{n *}, I^{n}+\Delta t I^{n *}, I_{A}^{n}+\Delta t I_{A}^{n *}, I_{D}^{n}+\Delta t I_{D}^{n *}, \\
I_{R}^{n}+\Delta t I_{R}^{n *}, I_{T}^{n}+\Delta t I_{T}^{n *}, R^{n}+\Delta t R^{n *}, D^{n}+\Delta t D^{n *}, V^{n}+\Delta t V^{n *}
\end{array}\right) \\
-t_{n}^{2-\beta\left(t_{n}\right)}\left(-\frac{\beta\left(t_{n+1}\right)-\beta\left(t_{n}\right)}{\Delta t} \ln t_{n}+\frac{2-\beta\left(t_{n}\right)}{t_{n}}\right) \\
\times V^{*}\left(t_{n}, S^{n}, I^{n}, I_{A}^{n}, I_{D}^{n}, I_{R}^{n}, I_{T}^{n}, R^{n}, D^{n}, V^{n}\right)
\end{array}\right]
$$




$$
\left.+\frac{\alpha}{M(\alpha)}\left\{\begin{array}{c}
\frac{23}{12} t_{n}^{2-\beta\left(t_{n}\right)}\left(-\frac{\beta\left(t_{n+1}\right)-\beta\left(t_{n}\right)}{\Delta t} \ln t_{n}+\frac{2-\beta\left(t_{n}\right)}{t_{n}}\right) \\
\times R^{*}\left(t_{n}, S^{n}, I^{n}, I_{A}^{n}, I_{D}^{n}, I_{R}^{n}, I_{T}^{n}, R^{n}, D^{n}, V^{n}\right) \Delta t \\
-\frac{4}{3} t_{n-1}^{2-\beta\left(t_{n-1}\right)}\left(-\frac{\beta\left(t_{n}\right)-\beta\left(t_{n-1}\right)}{\Delta t} \ln t_{n-1}+\frac{2-\beta\left(t_{n-1}\right)}{t_{n-1}}\right) \\
\times V^{*}\left(\begin{array}{c}
t_{n-1}, S^{n}-\Delta t S^{n *}, I^{n}-\Delta t I^{n *}, I_{A}^{n}-\Delta t I_{A}^{n *}, \\
I_{D}^{n}-\Delta t I_{D}^{n *}, I_{R}^{n}-\Delta t I_{R}^{n *}, I_{T}^{n}-\Delta t I_{T}^{n *}, \\
R^{n}-\Delta t R^{n *}, D^{n}-\Delta t D^{n *}, V^{n}-\Delta t V^{n *}
\end{array}\right) \Delta t \\
+\frac{5}{12} t_{n-2}^{2-\beta\left(t_{n-2}\right)}\left(-\frac{\beta\left(t_{n-1}\right)-\beta\left(t_{n-2}\right)}{\Delta t} \ln t_{n-2}+\frac{2-\beta\left(t_{n-2}\right)}{t_{n-2}}\right) \\
t_{n-2}, S^{n}-\Delta t S^{n *}-\Delta t S^{(n-1) *}, I^{n}-\Delta t I^{n *}-\Delta t I^{(n-1) *}, \\
I_{A}^{n}-\Delta t I_{A}^{n *}-\Delta t I_{A}^{(n-1) *}, I_{D}^{n}-\Delta t I_{D}^{n *}-\Delta t I_{D}^{(n-1) *}, \\
I_{R}^{n}-\Delta t I_{R}^{n *}-\Delta t I_{R}^{(n-1) *}, I_{T}^{n}-\Delta t I_{T}^{n *}-\Delta t I_{T}^{(n-1) *}, \\
R^{n}-\Delta t R^{n *}-\Delta t R^{(n-1) *}, D^{n}-\Delta t D^{n *}-\Delta t D^{(n-1) *}, \\
V^{n}-\Delta t V^{n *}-\Delta t V^{(n-1) *}
\end{array}\right) \Delta t\right\} .
$$

For the Atangana-Baleanu fractal-fractional derivative, we can have the following numerical scheme:

$$
\begin{aligned}
& S^{n+1}=\frac{1-\alpha}{A B(\alpha)} t_{n+1}^{2-\beta\left(t_{n+1}\right)}\left(-\frac{\beta\left(t_{n+2}\right)-\beta\left(t_{n+1}\right)}{\Delta t} \ln t_{n+1}+\frac{2-\beta\left(t_{n+1}\right)}{t_{n+1}}\right) \\
& \times S^{*}\left(\begin{array}{c}
t_{n+1}, S^{n}+\Delta t S^{n *}, I^{n}+\Delta t I^{n *}, I_{A}^{n}+\Delta t I_{A}^{n *}, \\
I_{D}^{n}+\Delta t I_{D}^{n *}, I_{R}^{n}+\Delta t I_{R}^{n *}, I_{T}^{n}+\Delta t I_{T}^{n *} \\
R^{n}+\Delta t R^{n *}, D^{n}+\Delta t D^{n *}, V^{n}+\Delta t V^{n *}
\end{array}\right) \\
& +\frac{\alpha(\Delta t)^{\alpha}}{A B(\alpha) \Gamma(\alpha+1)} \sum_{j=2}^{n} t_{j-2}^{2-\beta\left(t_{j-2}\right)}\left(-\frac{\beta\left(t_{j-1}\right)-\beta\left(t_{j-2}\right)}{\Delta t} \ln t_{j-2}+\frac{2-\beta\left(t_{j-2}\right)}{t_{j-2}}\right) \\
& \times S^{*}\left(\begin{array}{c}
t_{j-2}, S^{j}-\Delta t S^{j *}-\Delta t S^{(j-1) *}, I^{j}-\Delta t I^{j *}-\Delta t I^{(j-1) *}, \\
I_{A}^{j}-\Delta t I_{A}^{j *}-\Delta t I_{A}^{(j-1) *}, I_{D}^{j}-\Delta t I_{D}^{j *}-\Delta t I_{D}^{(j-1) *}, \\
I_{R}^{j}-\Delta t I_{R}^{j *}-\Delta t I_{R}^{(j-1) *}, I_{T}^{j}-\Delta t I_{T}^{j *}-\Delta t I_{T}^{(j-1) *}, \\
R^{j}-\Delta t R^{j *}-\Delta t R^{(j-1) *}, D^{j}-\Delta t D^{j *}-\Delta t D^{(j-1) *}, \\
V^{j}-\Delta t V^{j *}-\Delta t V^{(j-1) *}
\end{array}\right) \times \Pi \\
& +\frac{\alpha(\Delta t)^{\alpha}}{A B(\alpha) \Gamma(\alpha+2)} \\
& \times \sum_{j=2}^{n}\left[\begin{array}{c}
t_{j-1}^{2-\beta\left(t_{j-1}\right)}\left(-\frac{\beta\left(t_{j}\right)-\beta\left(t_{j-1}\right)}{\Delta t} \ln t_{j-1}+\frac{2-\beta\left(t_{j-1}\right)}{t_{j-1}}\right) \\
\times S^{*}\left(\begin{array}{c}
t_{j-1}, S^{j}-\Delta t S^{j *}, I^{j}-\Delta t I^{j *}, I_{A}^{j}-\Delta t I_{A}^{j *}, \\
I_{D}^{j}-\Delta t I_{D}^{j *}, I_{R}^{j}-\Delta t I_{R}^{j *}, I_{T}^{j}-\Delta t I_{T}^{j *}, \\
R^{j}-\Delta t R^{j *}, D^{j}-\Delta t D^{j *}, V^{j}-\Delta t V^{j *}
\end{array}\right) \\
-t_{j-2}^{2-\beta\left(t_{j-2}\right)}\left(-\frac{\beta\left(t_{j-1}\right)-\beta\left(t_{j-2}\right)}{\Delta t} \ln t_{j-2}+\frac{2-\beta\left(t_{j-2}\right)}{t_{j-2}}\right) \\
t_{j-2}, S^{j}-\Delta t S^{j *}-\Delta t S^{(j-1) *}, I^{j}-\Delta t I^{j *}-\Delta t I^{(j-1) *}, \\
I_{A}^{j}-\Delta t I_{A}^{j *}-\Delta t I_{A}^{(j-1) *}, I_{D}^{j}-\Delta t I_{D}^{j *}-\Delta t I_{D}^{(j-1) *}, \\
I_{R}^{j}-\Delta t I_{R}^{j *}-\Delta t I_{R}^{(j-1) *}, I_{T}^{j}-\Delta t I_{T}^{j *}-\Delta t I_{T}^{(j-1) *}, \\
R^{j}-\Delta t R^{j *}-\Delta t R^{(j-1) *}, D^{j}-\Delta t D^{j *}-\Delta t D^{(j-1) *}, \\
V^{j}-\Delta t V^{j *}-\Delta t V^{(j-1) *}
\end{array}\right] \times \Sigma
\end{aligned}
$$




$$
\begin{aligned}
& +\frac{\alpha(\Delta t)^{\alpha}}{2 A B(\alpha) \Gamma(\alpha+3)}
\end{aligned}
$$

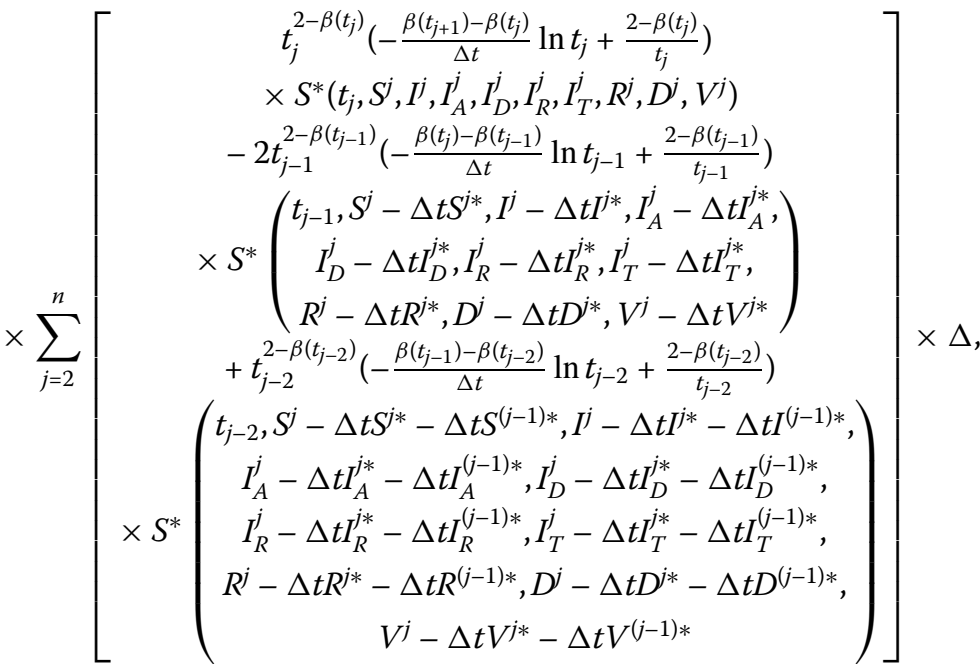

$$
\begin{aligned}
& I^{n+1}=\frac{1-\alpha}{A B(\alpha)} t_{n+1}^{2-\beta\left(t_{n+1}\right)}\left(-\frac{\beta\left(t_{n+2}\right)-\beta\left(t_{n+1}\right)}{\Delta t} \ln t_{n+1}+\frac{2-\beta\left(t_{n+1}\right)}{t_{n+1}}\right) \\
& \times I^{*}\left(\begin{array}{c}
t_{n+1}, S^{n}+\Delta t S^{n *}, I^{n}+\Delta t I^{n *}, I_{A}^{n}+\Delta t I_{A}^{n *}, \\
I_{D}^{n}+\Delta t I_{D}^{n *}, I_{R}^{n}+\Delta t I_{R}^{n *}, I_{T}^{n}+\Delta t I_{T}^{n *}, \\
R^{n}+\Delta t R^{n *}, D^{n}+\Delta t D^{n *}, V^{n}+\Delta t V^{n *}
\end{array}\right) \\
& +\frac{\alpha(\Delta t)^{\alpha}}{A B(\alpha) \Gamma(\alpha+1)} \sum_{j=2}^{n} t_{j-2}^{2-\beta\left(t_{j-2}\right)}\left(-\frac{\beta\left(t_{j-1}\right)-\beta\left(t_{j-2}\right)}{\Delta t} \ln t_{j-2}+\frac{2-\beta\left(t_{j-2}\right)}{t_{j-2}}\right) \\
& \times I^{*}\left(\begin{array}{c}
t_{j-2}, S^{j}-\Delta t S^{j *}-\Delta t S^{(j-1) *}, I^{j}-\Delta t I^{j *}-\Delta t I^{(j-1) *}, \\
I_{A}^{j}-\Delta t I_{A}^{j *}-\Delta t I_{A}^{(j-1) *}, I_{D}^{j}-\Delta t I_{D}^{j *}-\Delta t I_{D}^{(j-1) *}, \\
I_{R}^{j}-\Delta t I_{R}^{j *}-\Delta t I_{R}^{(j-1) *}, I_{T}^{j}-\Delta t I_{T}^{j *}-\Delta t I_{T}^{(j-1) *}, \\
R^{j}-\Delta t R^{j *}-\Delta t R^{(j-1) *}, D^{j}-\Delta t D^{j *}-\Delta t D^{(j-1) *}, \\
V^{j}-\Delta t V^{j *}-\Delta t V^{(j-1) *}
\end{array}\right) \times \Pi \\
& +\frac{\alpha(\Delta t)^{\alpha}}{A B(\alpha) \Gamma(\alpha+2)}
\end{aligned}
$$

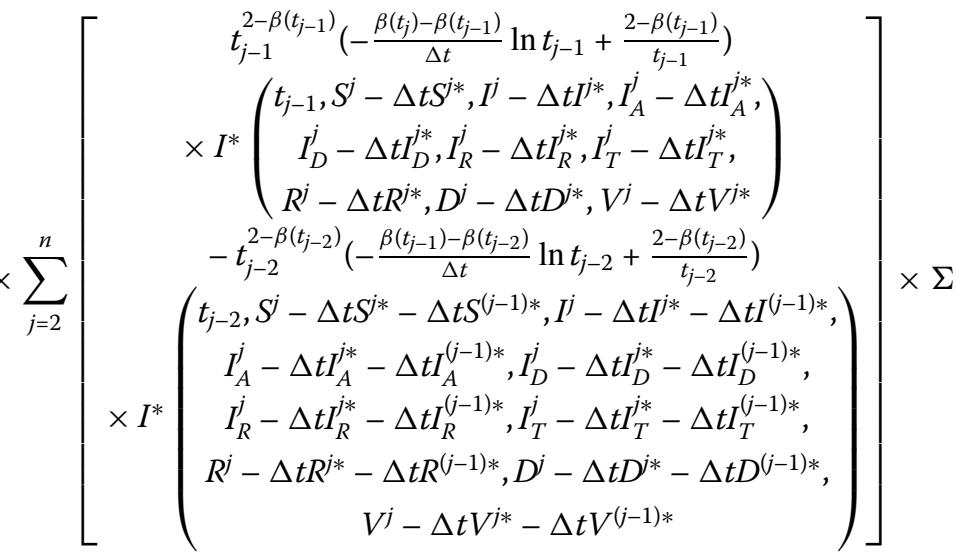

$$
\begin{aligned}
& +\frac{\alpha(\Delta t)^{\alpha}}{2 A B(\alpha) \Gamma(\alpha+3)}
\end{aligned}
$$




$$
\begin{aligned}
& \times \sum_{j=2}^{n}\left[\begin{array}{c}
t_{j}^{2-\beta\left(t_{j}\right)}\left(-\frac{\beta\left(t_{j+1}\right)-\beta\left(t_{j}\right)}{\Delta t} \ln t_{j}+\frac{2-\beta\left(t_{j}\right)}{t_{j}}\right) \\
\times I^{*}\left(t_{j}, S^{j}, I^{j}, I_{A}^{j}, I_{D}^{j}, I_{R}^{j}, I_{T}^{j}, R^{j}, D^{j}, V^{j}\right) \\
-2 t_{j-1}^{2-\beta\left(t_{j-1}\right)}\left(-\frac{\beta\left(t_{j}\right)-\beta\left(t_{j-1}\right)}{\Delta t} \ln t_{j-1}+\frac{2-\beta\left(t_{j-1}\right)}{t_{j-1}}\right) \\
\left(\begin{array}{c}
t_{j-1}, S^{j}-\Delta t S^{j *}, I^{j}-\Delta t I^{j *}, I_{A}^{j}-\Delta t I_{A}^{j *}, \\
I_{D}^{j}-\Delta t I_{D}^{j *}, I_{R}^{j}-\Delta t I_{R}^{j *}, I_{T}^{j}-\Delta t I_{T}^{j *}, \\
R^{j}-\Delta t R^{j *}, D^{j}-\Delta t D^{j *}, V^{j}-\Delta t V^{j *}
\end{array}\right) \\
\times I^{*}{ }^{2-\beta\left(t_{j-2}\right)}\left(-\frac{\beta\left(t_{j-1}\right)-\beta\left(t_{j-2}\right)}{\Delta t} \ln t_{j-2}+\frac{2-\beta\left(t_{j-2}\right)}{t_{j-2}}\right) \\
+t_{j-2}, S^{j}-\Delta t S^{j *}-\Delta t S^{(j-1) *}, I^{j}-\Delta t I^{j *}-\Delta t I^{(j-1) *}, \\
I_{A}^{j}-\Delta t I_{A}^{j *}-\Delta t I_{A}^{(j-1) *}, I_{D}^{j}-\Delta t I_{D}^{j *}-\Delta t I_{D}^{(j-1) *}, \\
I_{R}^{j}-\Delta t I_{R}^{j *}-\Delta t I_{R}^{(j-1) *}, I_{T}^{j}-\Delta t I_{T}^{j *}-\Delta t I_{T}^{(j-1) *}, \\
R^{j}-\Delta t R^{j *}-\Delta t R^{(j-1) *}, D^{j}-\Delta t D^{j *}-\Delta t D^{(j-1) *}, \\
V^{j}-\Delta t V^{j *}-\Delta t V^{(j-1) *}
\end{array}\right] \times \Delta, \\
& I_{A}^{n+1}=\frac{1-\alpha}{A B(\alpha)} t_{n+1}^{2-\beta\left(t_{n+1}\right)}\left(-\frac{\beta\left(t_{n+2}\right)-\beta\left(t_{n+1}\right)}{\Delta t} \ln t_{n+1}+\frac{2-\beta\left(t_{n+1}\right)}{t_{n+1}}\right) \\
& \times I_{A}^{*}\left(\begin{array}{c}
t_{n+1}, S^{n}+\Delta t S^{n *}, I^{n}+\Delta t I^{n *}, I_{A}^{n}+\Delta t I_{A}^{n *}, \\
I_{D}^{n}+\Delta t I_{D}^{n *}, I_{R}^{n}+\Delta t I_{R}^{n *}, I_{T}^{n}+\Delta t I_{T}^{n *}, \\
R^{n}+\Delta t R^{n *}, D^{n}+\Delta t D^{n *}, V^{n}+\Delta t V^{n *}
\end{array}\right) \\
& +\frac{\alpha(\Delta t)^{\alpha}}{A B(\alpha) \Gamma(\alpha+1)} \sum_{j=2}^{n} t_{j-2}^{2-\beta\left(t_{j-2}\right)}\left(-\frac{\beta\left(t_{j-1}\right)-\beta\left(t_{j-2}\right)}{\Delta t} \ln t_{j-2}+\frac{2-\beta\left(t_{j-2}\right)}{t_{j-2}}\right) \\
& \times I_{A}^{*}\left(\begin{array}{c}
t_{j-2}, S^{j}-\Delta t S^{j *}-\Delta t S^{(j-1) *}, I^{j}-\Delta t I^{j *}-\Delta t I^{(j-1) *}, \\
I_{A}^{j}-\Delta t I_{A}^{j *}-\Delta t I_{A}^{(j-1) *}, I_{D}^{j}-\Delta t I_{D}^{j *}-\Delta t I_{D}^{(j-1) *}, \\
I_{R}^{j}-\Delta t I_{R}^{j *}-\Delta t I_{R}^{(j-1) *}, I_{T}^{j}-\Delta t I_{T}^{j *}-\Delta t I_{T}^{(j-1) *}, \\
R^{j}-\Delta t R^{j *}-\Delta t R^{(j-1) *}, D^{j}-\Delta t D^{j *}-\Delta t D^{(j-1) *}, \\
V^{j}-\Delta t V^{j *}-\Delta t V^{(j-1) *}
\end{array}\right) \times \Pi \\
& +\frac{\alpha(\Delta t)^{\alpha}}{A B(\alpha) \Gamma(\alpha+2)}
\end{aligned}
$$

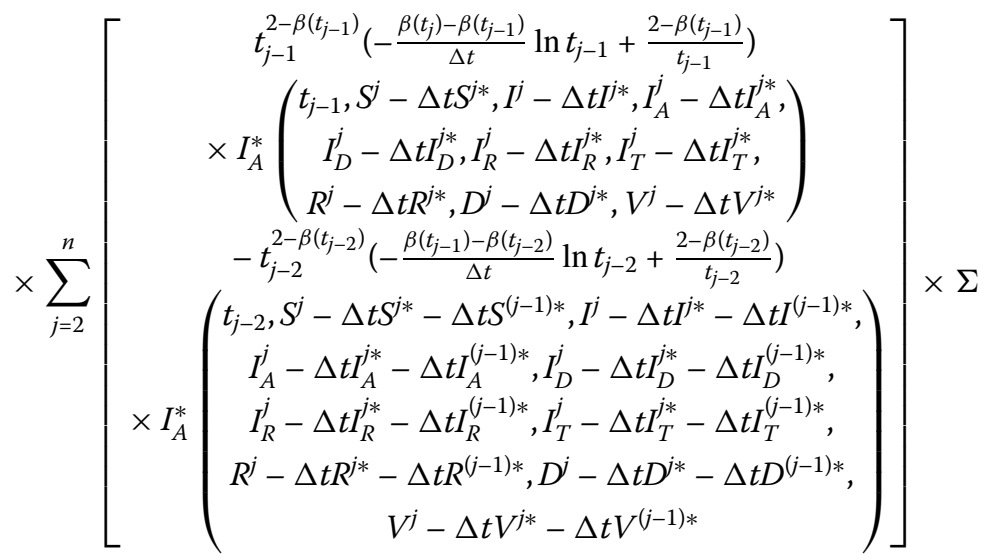

$$
\begin{aligned}
& +\frac{\alpha(\Delta t)^{\alpha}}{2 A B(\alpha) \Gamma(\alpha+3)}
\end{aligned}
$$




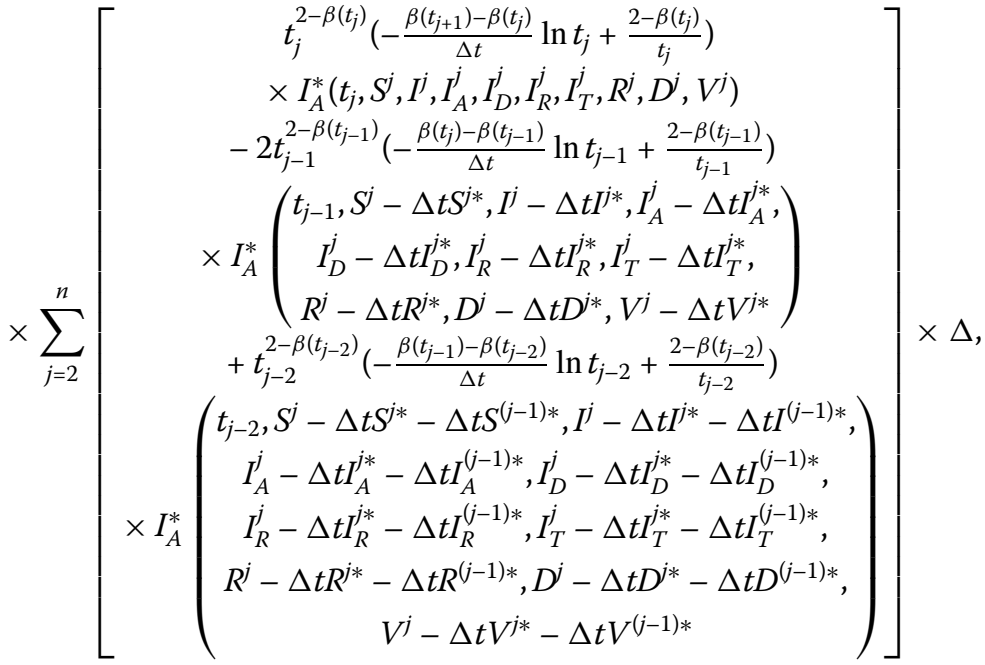

$$
\begin{aligned}
& I_{D}^{n+1}=\frac{1-\alpha}{A B(\alpha)} t_{n+1}^{2-\beta\left(t_{n+1}\right)}\left(-\frac{\beta\left(t_{n+2}\right)-\beta\left(t_{n+1}\right)}{\Delta t} \ln t_{n+1}+\frac{2-\beta\left(t_{n+1}\right)}{t_{n+1}}\right) \\
& \times I_{D}^{*}\left(\begin{array}{c}
t_{n+1}, S^{n}+\Delta t S^{n *}, I^{n}+\Delta t I^{n *}, I_{A}^{n}+\Delta t I_{A}^{n *}, \\
I_{D}^{n}+\Delta t I_{D}^{n *}, I_{R}^{n}+\Delta t I_{R}^{n *}, I_{T}^{n}+\Delta t I_{T}^{n *}, \\
R^{n}+\Delta t R^{n *}, D^{n}+\Delta t D^{n *}, V^{n}+\Delta t V^{n *}
\end{array}\right) \\
& +\frac{\alpha(\Delta t)^{\alpha}}{A B(\alpha) \Gamma(\alpha+1)} \sum_{j=2}^{n} t_{j-2}^{2-\beta\left(t_{j-2}\right)}\left(-\frac{\beta\left(t_{j-1}\right)-\beta\left(t_{j-2}\right)}{\Delta t} \ln t_{j-2}+\frac{2-\beta\left(t_{j-2}\right)}{t_{j-2}}\right) \\
& \times I_{D}^{*}\left(\begin{array}{c}
t_{j-2}, S^{j}-\Delta t S^{j *}-\Delta t S^{(j-1) *}, I^{j}-\Delta t I^{j *}-\Delta t I^{(j-1) *}, \\
I_{A}^{j}-\Delta t I_{A}^{j *}-\Delta t I_{A}^{(j-1) *}, I_{D}^{j}-\Delta t I_{D}^{j *}-\Delta t I_{D}^{(j-1) *}, \\
I_{R}^{j}-\Delta t I_{R}^{j *}-\Delta t I_{R}^{(j-1) *}, I_{T}^{j}-\Delta t I_{T}^{j *}-\Delta t I_{T}^{(j-1) *}, \\
R^{j}-\Delta t R^{j *}-\Delta t R^{(j-1) *}, D^{j}-\Delta t D^{j *}-\Delta t D^{(j-1) *}, \\
V^{j}-\Delta t V^{j *}-\Delta t V^{(j-1) *}
\end{array}\right) \times \Pi \\
& +\frac{\alpha(\Delta t)^{\alpha}}{A B(\alpha) \Gamma(\alpha+2)} \\
& \times \sum_{j=2}^{n}\left[\begin{array}{c}
t_{j-1}^{2-\beta\left(t_{j-1}\right)}\left(-\frac{\beta\left(t_{j}\right)-\beta\left(t_{j-1}\right)}{\Delta t} \ln t_{j-1}+\frac{2-\beta\left(t_{j-1}\right)}{t_{j-1}}\right) \\
\left(\begin{array}{c}
t_{j-1}, S^{j}-\Delta t S^{j *}, I^{j}-\Delta t I^{j *}, I_{A}^{j}-\Delta t I_{A}^{j *}, \\
I_{D}^{j}-\Delta t I_{D}^{j *}, I_{R}^{j}-\Delta t I_{R}^{j *}, I_{T}^{j}-\Delta t I_{T}^{j *}, \\
R^{j}-\Delta t R^{j *}, D^{j}-\Delta t D^{j *}, V^{j}-\Delta t V^{j *}
\end{array}\right) \\
-t_{j-2}^{2-\beta\left(t_{j-2}\right)}\left(-\frac{\beta\left(t_{j-1}\right)-\beta\left(t_{j-2}\right)}{\Delta t} \ln t_{j-2}+\frac{2-\beta\left(t_{j-2}\right)}{t_{j-2}}\right) \\
t_{j-2}, S^{j}-\Delta t S^{j *}-\Delta t S^{(j-1) *}, I^{j}-\Delta t I^{j *}-\Delta t I^{(j-1) *}, \\
I_{A}^{j}-\Delta t I_{A}^{j *}-\Delta t I_{A}^{(j-1) *}, I_{D}^{j}-\Delta t I_{D}^{j *}-\Delta t I_{D}^{(j-1) *}, \\
I_{R}^{j}-\Delta t I_{R}^{j *}-\Delta t I_{R}^{(j-1) *}, I_{T}^{j}-\Delta t I_{T}^{j *}-\Delta t I_{T}^{(j-1) *}, \\
R^{j}-\Delta t R^{j *}-\Delta t R^{(j-1) *}, D^{j}-\Delta t D^{j *}-\Delta t D^{(j-1) *}, \\
V^{j}-\Delta t V^{j *}-\Delta t V^{(j-1) *}
\end{array}\right] \times \Sigma \\
& +\frac{\alpha(\Delta t)^{\alpha}}{2 A B(\alpha) \Gamma(\alpha+3)}
\end{aligned}
$$




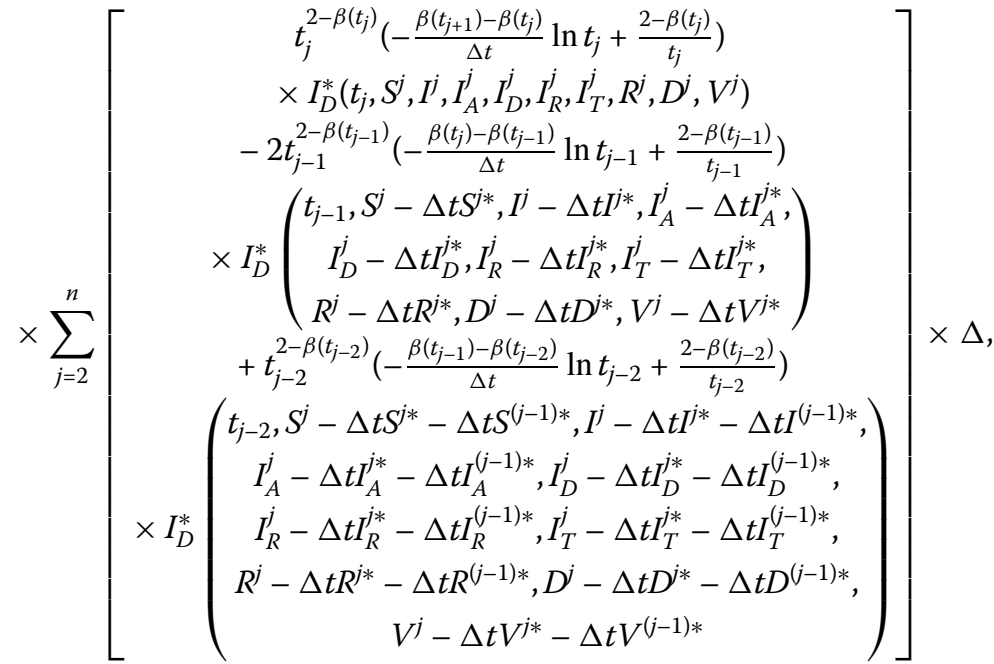

$$
\begin{aligned}
& I_{R}^{n+1}=\frac{1-\alpha}{A B(\alpha)} t_{n+1}^{2-\beta\left(t_{n+1}\right)}\left(-\frac{\beta\left(t_{n+2}\right)-\beta\left(t_{n+1}\right)}{\Delta t} \ln t_{n+1}+\frac{2-\beta\left(t_{n+1}\right)}{t_{n+1}}\right) \\
& \times I_{R}^{*}\left(\begin{array}{c}
t_{n+1}, S^{n}+\Delta t S^{n *}, I^{n}+\Delta t I^{n *}, I_{A}^{n}+\Delta t I_{A}^{n *}, \\
I_{D}^{n}+\Delta t I_{D}^{n *}, I_{R}^{n}+\Delta t I_{R}^{n *}, I_{T}^{n}+\Delta t I_{T}^{n *}, \\
R^{n}+\Delta t R^{n *}, D^{n}+\Delta t D^{n *}, V^{n}+\Delta t V^{n *}
\end{array}\right) \\
& +\frac{\alpha(\Delta t)^{\alpha}}{A B(\alpha) \Gamma(\alpha+1)} \sum_{j=2}^{n} t_{j-2}^{2-\beta\left(t_{j-2}\right)}\left(-\frac{\beta\left(t_{j-1}\right)-\beta\left(t_{j-2}\right)}{\Delta t} \ln t_{j-2}+\frac{2-\beta\left(t_{j-2}\right)}{t_{j-2}}\right) \\
& \times I_{R}^{*}\left(\begin{array}{c}
t_{j-2}, S^{j}-\Delta t S^{j *}-\Delta t S^{(j-1) *}, I^{j}-\Delta t I^{j *}-\Delta t I^{(j-1) *}, \\
I_{A}^{j}-\Delta t I_{A}^{j *}-\Delta t I_{A}^{(j-1) *}, I_{D}^{j}-\Delta t I_{D}^{j *}-\Delta t I_{D}^{(j-1) *}, \\
I_{R}^{j}-\Delta t I_{R}^{j *}-\Delta t I_{R}^{(j-1) *}, I_{T}^{j}-\Delta t I_{T}^{j *}-\Delta t I_{T}^{(j-1) *}, \\
R^{j}-\Delta t R^{j *}-\Delta t R^{(j-1) *}, D^{j}-\Delta t D^{j *}-\Delta t D^{(j-1) *}, \\
V^{j}-\Delta t V^{j *}-\Delta t V^{(j-1) *}
\end{array}\right) \times \Pi \\
& +\frac{\alpha(\Delta t)^{\alpha}}{A B(\alpha) \Gamma(\alpha+2)} \\
& \times \sum_{j=2}^{n}\left[\begin{array}{c}
t_{j-1}^{2-\beta\left(t_{j-1}\right)}\left(-\frac{\beta\left(t_{j}\right)-\beta\left(t_{j-1}\right)}{\Delta t} \ln t_{j-1}+\frac{2-\beta\left(t_{j-1}\right)}{t_{j-1}}\right) \\
\left(\begin{array}{c}
t_{j-1}, S^{j}-\Delta t S^{j *}, I^{j}-\Delta t I^{j *}, I_{A}^{j}-\Delta t I_{A}^{j *}, \\
I_{D}^{j}-\Delta t I_{D}^{j *}, I_{R}^{j}-\Delta t I_{R}^{j *}, I_{T}^{j}-\Delta t I_{T}^{j *}, \\
R^{j}-\Delta t R^{j *}, D^{j}-\Delta t D^{j *}, V^{j}-\Delta t V^{j *}
\end{array}\right) \\
-t_{j-2}^{2-\beta\left(t_{j-2}\right)}\left(-\frac{\beta\left(t_{j-1}\right)-\beta\left(t_{j-2}\right)}{\Delta t} \ln t_{j-2}+\frac{2-\beta\left(t_{j-2}\right)}{t_{j-2}}\right) \\
t_{j-2}, S^{j}-\Delta t S^{j *}-\Delta t S^{(j-1) *}, I^{j}-\Delta t I^{j *}-\Delta t I^{(j-1) *}, \\
I_{A}^{j}-\Delta t I_{A}^{j *}-\Delta t I_{A}^{(j-1) *}, I_{D}^{j}-\Delta t I_{D}^{j *}-\Delta t I_{D}^{(j-1) *}, \\
I_{R}^{j}-\Delta t I_{R}^{j *}-\Delta t I_{R}^{(j-1) *}, I_{T}^{j}-\Delta t I_{T}^{j *}-\Delta t I_{T}^{(j-1) *}, \\
R^{j}-\Delta t R^{j *}-\Delta t R^{(j-1) *}, D^{j}-\Delta t D^{j *}-\Delta t D^{(j-1) *}, \\
V^{j}-\Delta t V^{j *}-\Delta t V^{(j-1) *}
\end{array}\right] \times \Sigma \\
& +\frac{\alpha(\Delta t)^{\alpha}}{2 A B(\alpha) \Gamma(\alpha+3)}
\end{aligned}
$$




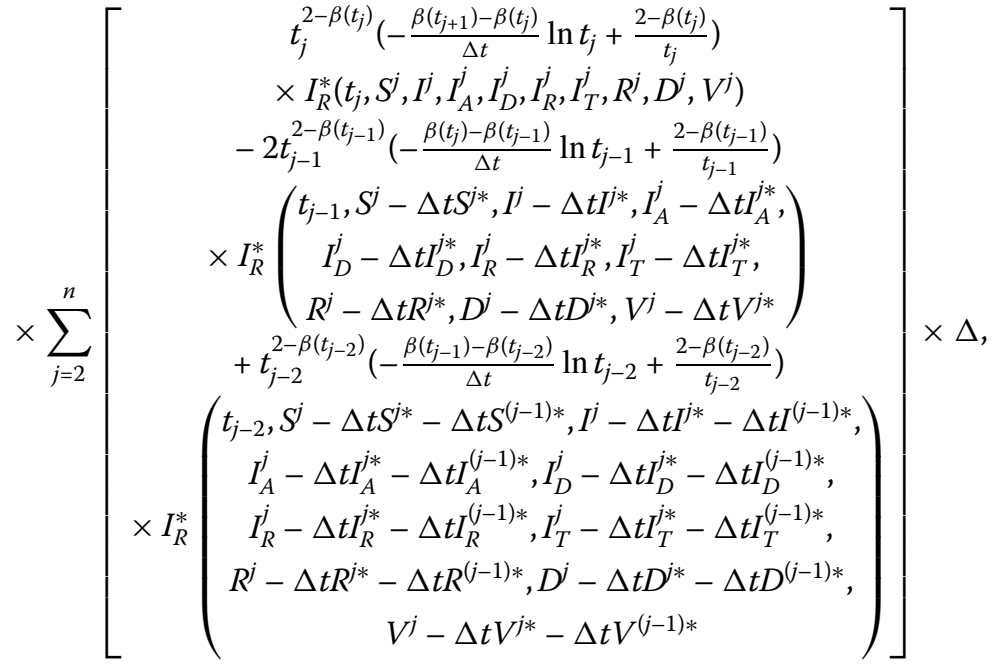

$$
\begin{aligned}
& I_{T}^{n+1}=\frac{1-\alpha}{A B(\alpha)} t_{n+1}^{2-\beta\left(t_{n+1}\right)}\left(-\frac{\beta\left(t_{n+2}\right)-\beta\left(t_{n+1}\right)}{\Delta t} \ln t_{n+1}+\frac{2-\beta\left(t_{n+1}\right)}{t_{n+1}}\right) \\
& \times I_{T}^{*}\left(\begin{array}{c}
t_{n+1}, S^{n}+\Delta t S^{n *}, I^{n}+\Delta t I^{n *}, I_{A}^{n}+\Delta t I_{A}^{n *}, \\
I_{D}^{n}+\Delta t I_{D}^{n *}, I_{R}^{n}+\Delta t I_{R}^{n *}, I_{T}^{n}+\Delta t I_{T}^{n *}, \\
R^{n}+\Delta t R^{n *}, D^{n}+\Delta t D^{n *}, V^{n}+\Delta t V^{n *}
\end{array}\right) \\
& +\frac{\alpha(\Delta t)^{\alpha}}{A B(\alpha) \Gamma(\alpha+1)} \sum_{j=2}^{n} t_{j-2}^{2-\beta\left(t_{j-2}\right)}\left(-\frac{\beta\left(t_{j-1}\right)-\beta\left(t_{j-2}\right)}{\Delta t} \ln t_{j-2}+\frac{2-\beta\left(t_{j-2}\right)}{t_{j-2}}\right) \\
& \times I_{T}^{*}\left(\begin{array}{c}
t_{j-2}, S^{j}-\Delta t S^{j *}-\Delta t S^{(j-1) *}, I^{j}-\Delta t I^{j *}-\Delta t I^{(j-1) *}, \\
I_{A}^{j}-\Delta t I_{A}^{j *}-\Delta t I_{A}^{(j-1) *}, I_{D}^{j}-\Delta t I_{D}^{j *}-\Delta t I_{D}^{(j-1) *}, \\
I_{R}^{j}-\Delta t I_{R}^{j *}-\Delta t I_{R}^{(j-1) *}, I_{T}^{j}-\Delta t I_{T}^{j *}-\Delta t I_{T}^{(j-1) *}, \\
R^{j}-\Delta t R^{j *}-\Delta t R^{(j-1) *}, D^{j}-\Delta t D^{j *}-\Delta t D^{(j-1) *}, \\
V^{j}-\Delta t V^{j *}-\Delta t V^{(j-1) *}
\end{array}\right) \times \Pi \\
& +\frac{\alpha(\Delta t)^{\alpha}}{A B(\alpha) \Gamma(\alpha+2)}
\end{aligned}
$$

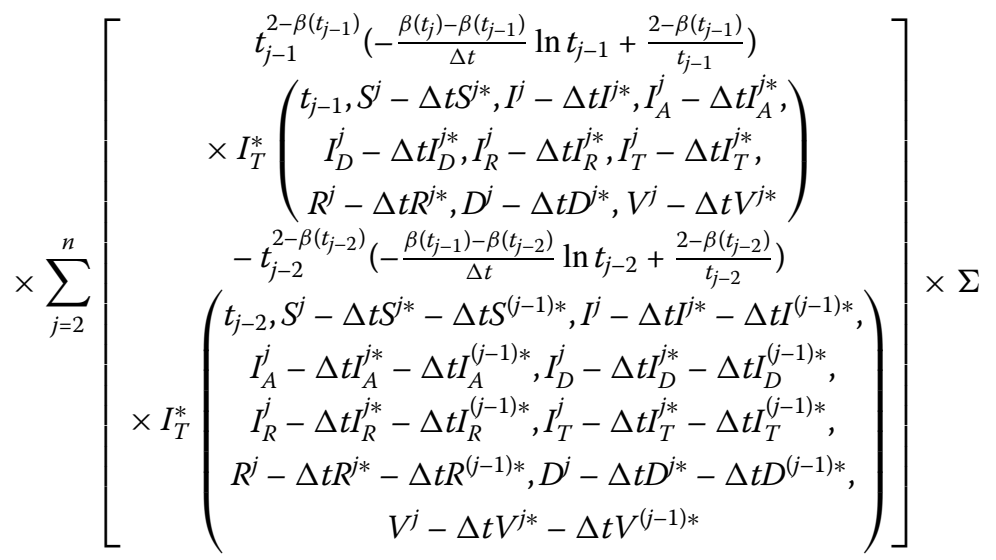

$$
\begin{aligned}
& +\frac{\alpha(\Delta t)^{\alpha}}{2 A B(\alpha) \Gamma(\alpha+3)}
\end{aligned}
$$




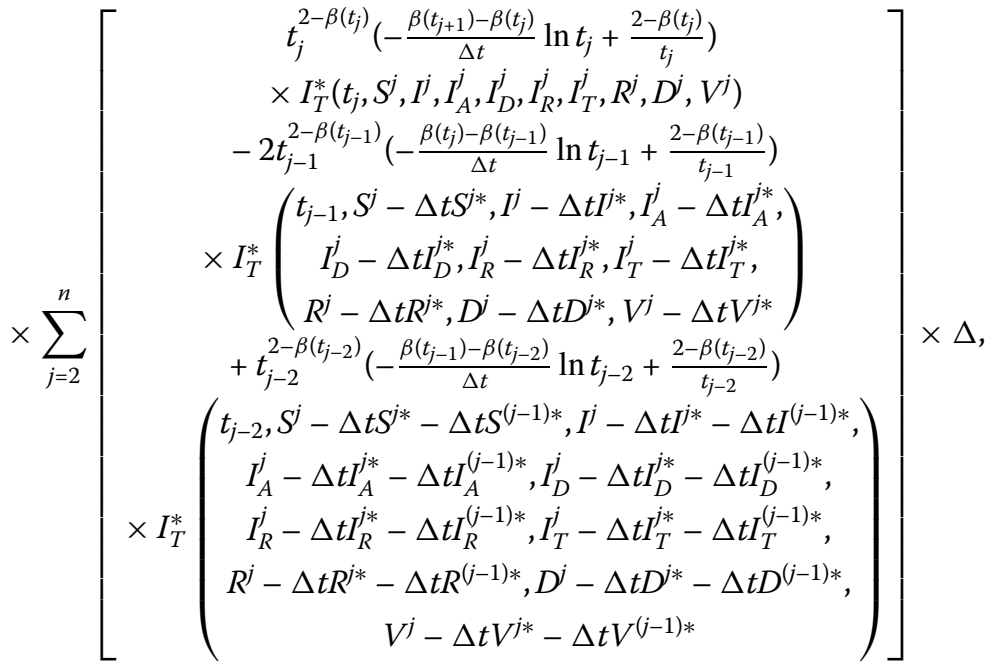

$$
\begin{aligned}
& R^{n+1}=\frac{1-\alpha}{A B(\alpha)} t_{n+1}^{2-\beta\left(t_{n+1}\right)}\left(-\frac{\beta\left(t_{n+2}\right)-\beta\left(t_{n+1}\right)}{\Delta t} \ln t_{n+1}+\frac{2-\beta\left(t_{n+1}\right)}{t_{n+1}}\right) \\
& \times R^{*}\left(\begin{array}{c}
t_{n+1}, S^{n}+\Delta t S^{n *}, I^{n}+\Delta t I^{n *}, I_{A}^{n}+\Delta t I_{A}^{n *}, \\
I_{D}^{n}+\Delta t I_{D}^{n *}, I_{R}^{n}+\Delta t I_{R}^{n *}, I_{T}^{n}+\Delta t I_{T}^{n *}, \\
R^{n}+\Delta t R^{n *}, D^{n}+\Delta t D^{n *}, V^{n}+\Delta t V^{n *}
\end{array}\right) \\
& +\frac{\alpha(\Delta t)^{\alpha}}{A B(\alpha) \Gamma(\alpha+1)} \sum_{j=2}^{n} t_{j-2}^{2-\beta\left(t_{j-2}\right)}\left(-\frac{\beta\left(t_{j-1}\right)-\beta\left(t_{j-2}\right)}{\Delta t} \ln t_{j-2}+\frac{2-\beta\left(t_{j-2}\right)}{t_{j-2}}\right) \\
& \times R^{*}\left(\begin{array}{c}
t_{j-2}, S^{j}-\Delta t S^{j *}-\Delta t S^{(j-1) *}, I^{j}-\Delta t I^{j *}-\Delta t I^{(j-1) *}, \\
I_{A}^{j}-\Delta t I_{A}^{j *}-\Delta t I_{A}^{(j-1) *}, I_{D}^{j}-\Delta t I_{D}^{j *}-\Delta t I_{D}^{(j-1) *}, \\
I_{R}^{j}-\Delta t I_{R}^{j *}-\Delta t I_{R}^{(j-1) *}, I_{T}^{j}-\Delta t I_{T}^{j *}-\Delta t I_{T}^{(j-1) *}, \\
R^{j}-\Delta t R^{j *}-\Delta t R^{(j-1) *}, D^{j}-\Delta t D^{j *}-\Delta t D^{(j-1) *}, \\
V^{j}-\Delta t V^{j *}-\Delta t V^{(j-1) *}
\end{array}\right) \times \Pi \\
& +\frac{\alpha(\Delta t)^{\alpha}}{A B(\alpha) \Gamma(\alpha+2)} \\
& \times \sum_{j=2}^{n}\left[\begin{array}{c}
t_{j-1}^{2-\beta\left(t_{j-1}\right)}\left(-\frac{\beta\left(t_{j}\right)-\beta\left(t_{j-1}\right)}{\Delta t} \ln t_{j-1}+\frac{2-\beta\left(t_{j-1}\right)}{t_{j-1}}\right) \\
\times R^{*}\left(\begin{array}{c}
t_{j-1}, S^{j}-\Delta t S^{j *}, I^{j}-\Delta t I^{j *}, I_{A}^{j}-\Delta t I_{A}^{j *}, \\
I_{D}^{j}-\Delta t I_{D}^{j *}, I_{R}^{j}-\Delta t I_{R}^{j *}, I_{T}^{j}-\Delta t I_{T}^{j *}, \\
R^{j}-\Delta t R^{j *}, D^{j}-\Delta t D^{j *}, V^{j}-\Delta t V^{j *}
\end{array}\right) \\
-t_{j-2}^{2-\beta\left(t_{j-2}\right)}\left(-\frac{\beta\left(t_{j-1}\right)-\beta\left(t_{j-2}\right)}{\Delta t} \ln t_{j-2}+\frac{2-\beta\left(t_{j-2}\right)}{t_{j-2}}\right) \\
t_{j-2}, S^{j}-\Delta t S^{j *}-\Delta t S^{(j-1) *}, I^{j}-\Delta t I^{j *}-\Delta t I^{(j-1) *}, \\
I_{A}^{j}-\Delta t I_{A}^{j *}-\Delta t I_{A}^{(j-1) *}, I_{D}^{j}-\Delta t I_{D}^{j *}-\Delta t I_{D}^{(j-1) *}, \\
I_{R}^{j}-\Delta t I_{R}^{j *}-\Delta t I_{R}^{(j-1) *}, I_{T}^{j}-\Delta t I_{T}^{j *}-\Delta t I_{T}^{(j-1) *}, \\
R^{j}-\Delta t R^{j *}-\Delta t R^{(j-1) *}, D^{j}-\Delta t D^{j *}-\Delta t D^{(j-1) *}, \\
V^{j}-\Delta t V^{j *}-\Delta t V^{(j-1) *}
\end{array}\right] \times \Sigma \\
& +\frac{\alpha(\Delta t)^{\alpha}}{2 A B(\alpha) \Gamma(\alpha+3)}
\end{aligned}
$$




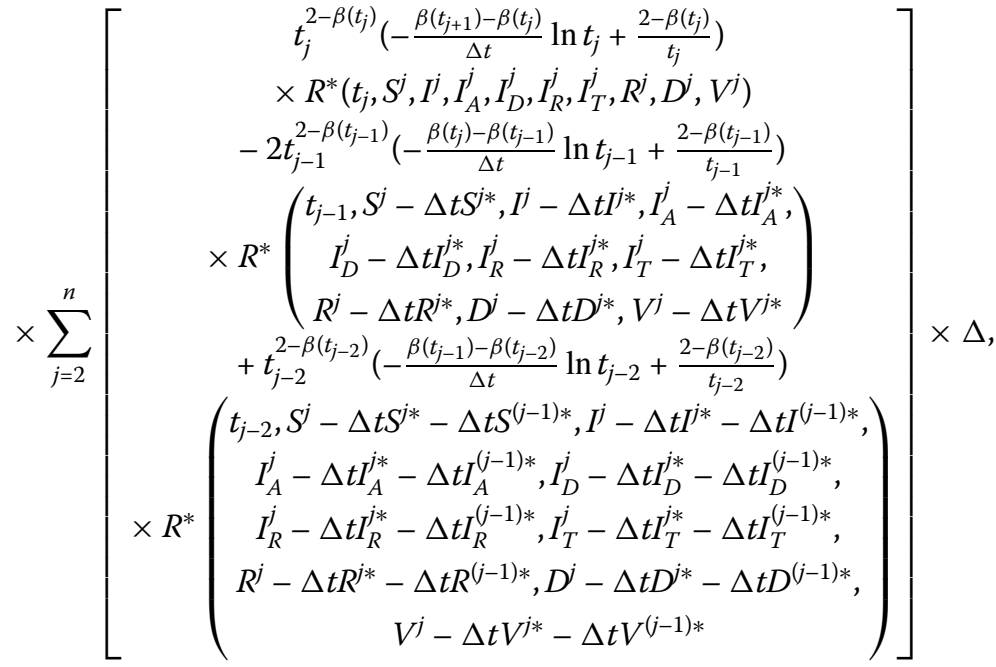

$$
\begin{aligned}
& D^{n+1}=\frac{1-\alpha}{A B(\alpha)} t_{n+1}^{2-\beta\left(t_{n+1}\right)}\left(-\frac{\beta\left(t_{n+2}\right)-\beta\left(t_{n+1}\right)}{\Delta t} \ln t_{n+1}+\frac{2-\beta\left(t_{n+1}\right)}{t_{n+1}}\right) \\
& \times D^{*}\left(\begin{array}{c}
t_{n+1}, S^{n}+\Delta t S^{n *}, I^{n}+\Delta t I^{n *}, I_{A}^{n}+\Delta t I_{A}^{n *}, \\
I_{D}^{n}+\Delta t I_{D}^{n *}, I_{R}^{n}+\Delta t I_{R}^{n *}, I_{T}^{n}+\Delta t I_{T}^{n *}, \\
R^{n}+\Delta t R^{n *}, D^{n}+\Delta t D^{n *}, V^{n}+\Delta t V^{n *}
\end{array}\right) \\
& +\frac{\alpha(\Delta t)^{\alpha}}{A B(\alpha) \Gamma(\alpha+1)} \sum_{j=2}^{n} t_{j-2}^{2-\beta\left(t_{j-2}\right)}\left(-\frac{\beta\left(t_{j-1}\right)-\beta\left(t_{j-2}\right)}{\Delta t} \ln t_{j-2}+\frac{2-\beta\left(t_{j-2}\right)}{t_{j-2}}\right) \\
& \times D^{*}\left(\begin{array}{c}
t_{j-2}, S^{j}-\Delta t S^{j *}-\Delta t S^{(j-1) *}, I^{j}-\Delta t I^{j *}-\Delta t I^{(j-1) *}, \\
I_{A}^{j}-\Delta t I_{A}^{j *}-\Delta t I_{A}^{(j-1) *}, I_{D}^{j}-\Delta t I_{D}^{j *}-\Delta t I_{D}^{(j-1) *}, \\
I_{R}^{j}-\Delta t I_{R}^{j *}-\Delta t I_{R}^{(j-1) *}, I_{T}^{j}-\Delta t I_{T}^{j *}-\Delta t I_{T}^{(j-1) *}, \\
R^{j}-\Delta t R^{j *}-\Delta t R^{(j-1) *}, D^{j}-\Delta t D^{j *}-\Delta t D^{(j-1) *}, \\
V^{j}-\Delta t V^{j *}-\Delta t V^{(j-1) *}
\end{array}\right) \times \Pi \\
& +\frac{\alpha(\Delta t)^{\alpha}}{A B(\alpha) \Gamma(\alpha+2)} \\
& \times \sum_{j=2}^{n}\left[\begin{array}{c}
t_{j-1}^{2-\beta\left(t_{j-1}\right)}\left(-\frac{\beta\left(t_{j}\right)-\beta\left(t_{j-1}\right)}{\Delta t} \ln t_{j-1}+\frac{2-\beta\left(t_{j-1}\right)}{t_{j-1}}\right) \\
\times D^{*}\left(\begin{array}{c}
t_{j-1}, S^{j}-\Delta t S^{j *}, I^{j}-\Delta t I^{j *}, I_{A}^{j}-\Delta t I_{A}^{j *}, \\
I_{D}^{j}-\Delta t I_{D}^{j *}, I_{R}^{j}-\Delta t I_{R}^{j *}, I_{T}^{j}-\Delta t I_{T}^{j *}, \\
R^{j}-\Delta t R^{j *}, D^{j}-\Delta t D^{j *}, V^{j}-\Delta t V^{j *}
\end{array}\right) \\
-t_{j-2}^{2-\beta\left(t_{j-2}\right)}\left(-\frac{\beta\left(t_{j-1}\right)-\beta\left(t_{j-2}\right)}{\Delta t} \ln t_{j-2}+\frac{2-\beta\left(t_{j-2}\right)}{t_{j-2}}\right) \\
t_{j-2}, S^{j}-\Delta t S^{j *}-\Delta t S^{(j-1) *}, I^{j}-\Delta t I^{j *}-\Delta t I^{(j-1) *}, \\
I_{A}^{j}-\Delta t I_{A}^{j *}-\Delta t I_{A}^{(j-1) *}, I_{D}^{j}-\Delta t I_{D}^{j *}-\Delta t I_{D}^{(j-1) *}, \\
I_{R}^{j}-\Delta t I_{R}^{j *}-\Delta t I_{R}^{(j-1) *}, I_{T}^{j}-\Delta t I_{T}^{j *}-\Delta t I_{T}^{(j-1) *}, \\
R^{j}-\Delta t R^{j *}-\Delta t R^{(j-1) *}, D^{j}-\Delta t D^{j *}-\Delta t D^{(j-1) *}, \\
V^{j}-\Delta t V^{j *}-\Delta t V^{(j-1) *}
\end{array}\right) \times \Sigma \\
& +\frac{\alpha(\Delta t)^{\alpha}}{2 A B(\alpha) \Gamma(\alpha+3)}
\end{aligned}
$$




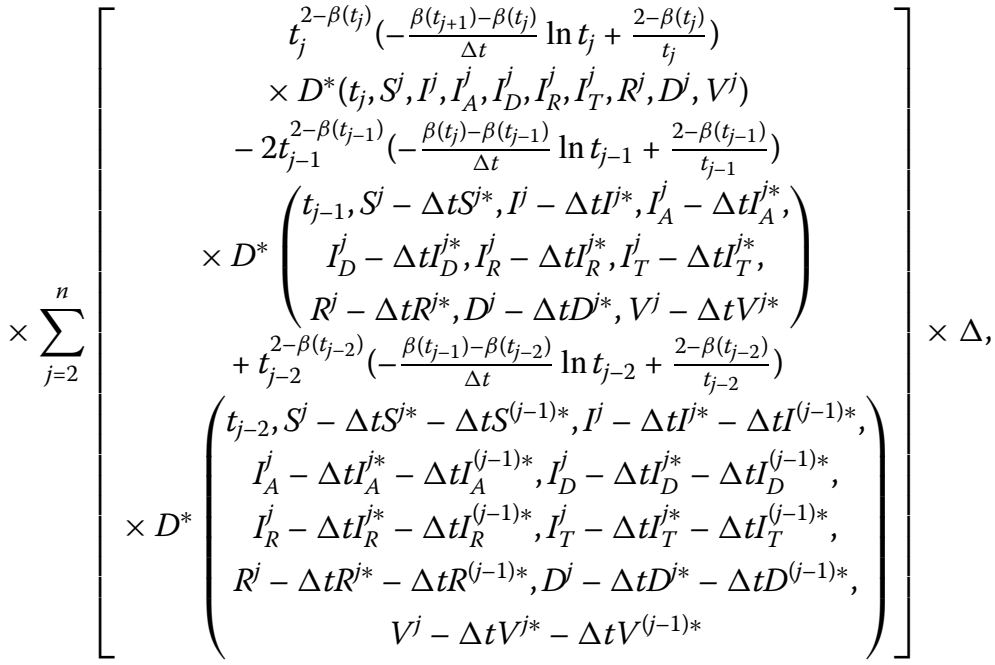

$$
\begin{aligned}
& V^{n+1}=\frac{1-\alpha}{A B(\alpha)} t_{n+1}^{2-\beta\left(t_{n+1}\right)}\left(-\frac{\beta\left(t_{n+2}\right)-\beta\left(t_{n+1}\right)}{\Delta t} \ln t_{n+1}+\frac{2-\beta\left(t_{n+1}\right)}{t_{n+1}}\right) \\
& \times V^{*}\left(\begin{array}{c}
t_{n+1}, S^{n}+\Delta t S^{n *}, I^{n}+\Delta t I^{n *}, I_{A}^{n}+\Delta t I_{A}^{n *}, \\
I_{D}^{n}+\Delta t I_{D}^{n *}, I_{R}^{n}+\Delta t I_{R}^{n *}, I_{T}^{n}+\Delta t I_{T}^{n *}, \\
R^{n}+\Delta t R^{n *}, D^{n}+\Delta t D^{n *}, V^{n}+\Delta t V^{n *}
\end{array}\right) \\
& +\frac{\alpha(\Delta t)^{\alpha}}{A B(\alpha) \Gamma(\alpha+1)} \sum_{j=2}^{n} t_{j-2}^{2-\beta\left(t_{j-2}\right)}\left(-\frac{\beta\left(t_{j-1}\right)-\beta\left(t_{j-2}\right)}{\Delta t} \ln t_{j-2}+\frac{2-\beta\left(t_{j-2}\right)}{t_{j-2}}\right) \\
& \times V^{*}\left(\begin{array}{c}
t_{j-2}, S^{j}-\Delta t S^{j *}-\Delta t S^{(j-1) *}, I^{j}-\Delta t I^{j *}-\Delta t I^{(j-1) *}, \\
I_{A}^{j}-\Delta t I_{A}^{j *}-\Delta t I_{A}^{(j-1) *}, I_{D}^{j}-\Delta t I_{D}^{j *}-\Delta t I_{D}^{(j-1) *}, \\
I_{R}^{j}-\Delta t I_{R}^{j *}-\Delta t I_{R}^{(j-1) *}, I_{T}^{j}-\Delta t I_{T}^{j *}-\Delta t I_{T}^{(j-1) *}, \\
R^{j}-\Delta t R^{j *}-\Delta t R^{(j-1) *}, D^{j}-\Delta t D^{j *}-\Delta t D^{(j-1) *}, \\
V^{j}-\Delta t V^{j *}-\Delta t V^{(j-1) *}
\end{array}\right) \times \Pi \\
& +\frac{\alpha(\Delta t)^{\alpha}}{A B(\alpha) \Gamma(\alpha+2)}
\end{aligned}
$$

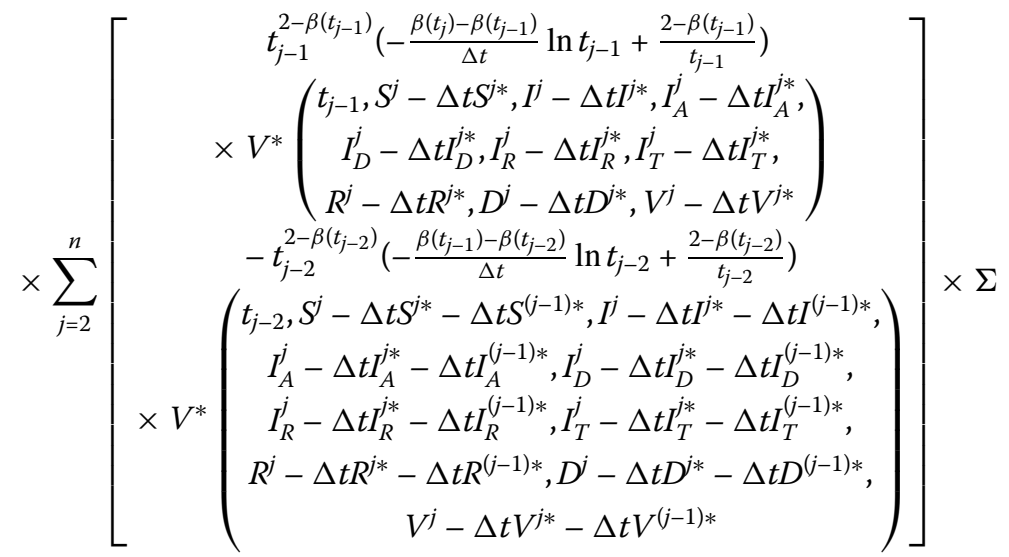

$$
\begin{aligned}
& +\frac{\alpha(\Delta t)^{\alpha}}{2 A B(\alpha) \Gamma(\alpha+3)}
\end{aligned}
$$




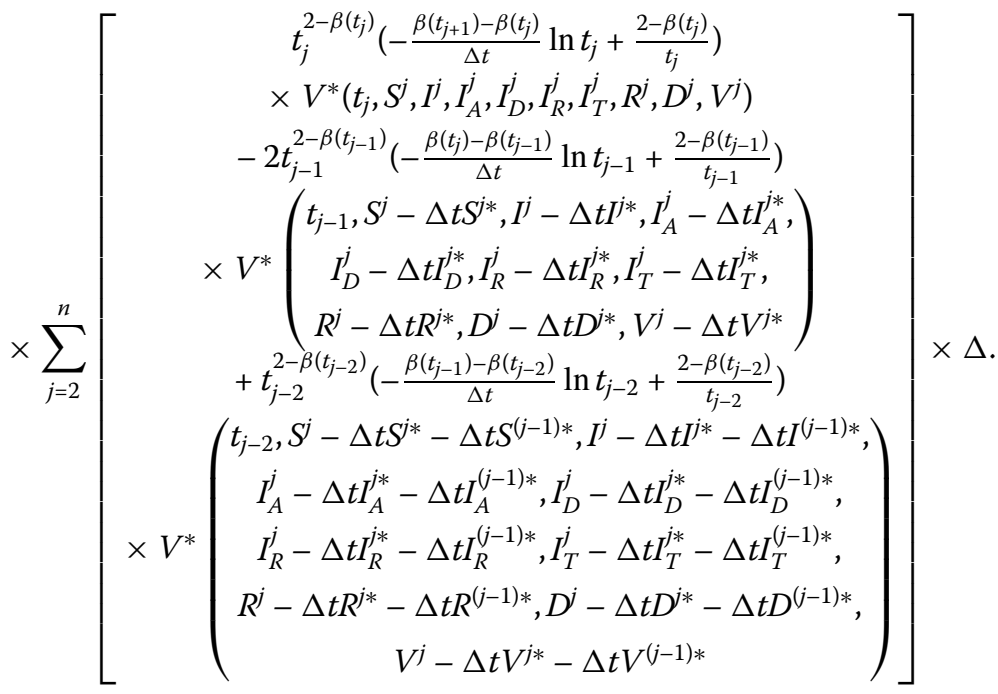

For the power-law kernel, we can have the following:

$$
\begin{aligned}
& S^{n+1}=\frac{(\Delta t)^{\alpha}}{\Gamma(\alpha+1)} \sum_{j=2}^{n} t_{j-2}^{2-\beta\left(t_{j-2}\right)}\left(-\frac{\beta\left(t_{j-1}\right)-\beta\left(t_{j-2}\right)}{\Delta t} \ln t_{j-2}+\frac{2-\beta\left(t_{j-2}\right)}{t_{j-2}}\right) \\
& \times S^{*}\left(\begin{array}{c}
t_{j-2}, S^{j}-\Delta t S^{j *}-\Delta t S^{(j-1) *}, I^{j}-\Delta t I^{j *}-\Delta t I^{(j-1) *}, \\
I_{A}^{j}-\Delta t I_{A}^{j *}-\Delta t I_{A}^{(j-1) *}, I_{D}^{j}-\Delta t I_{D}^{j *}-\Delta t I_{D}^{(j-1) *}, \\
I_{R}^{j}-\Delta t I_{R}^{j *}-\Delta t I_{R}^{(j-1) *}, I_{T}^{j}-\Delta t I_{T}^{j *}-\Delta t I_{T}^{(j-1) *}, \\
R^{j}-\Delta t R^{j *}-\Delta t R^{(j-1) *}, D^{j}-\Delta t D^{j *}-\Delta t D^{(j-1) *}, \\
V^{j}-\Delta t V^{j *}-\Delta t V^{(j-1) *}
\end{array}\right) \times \Pi
\end{aligned}
$$

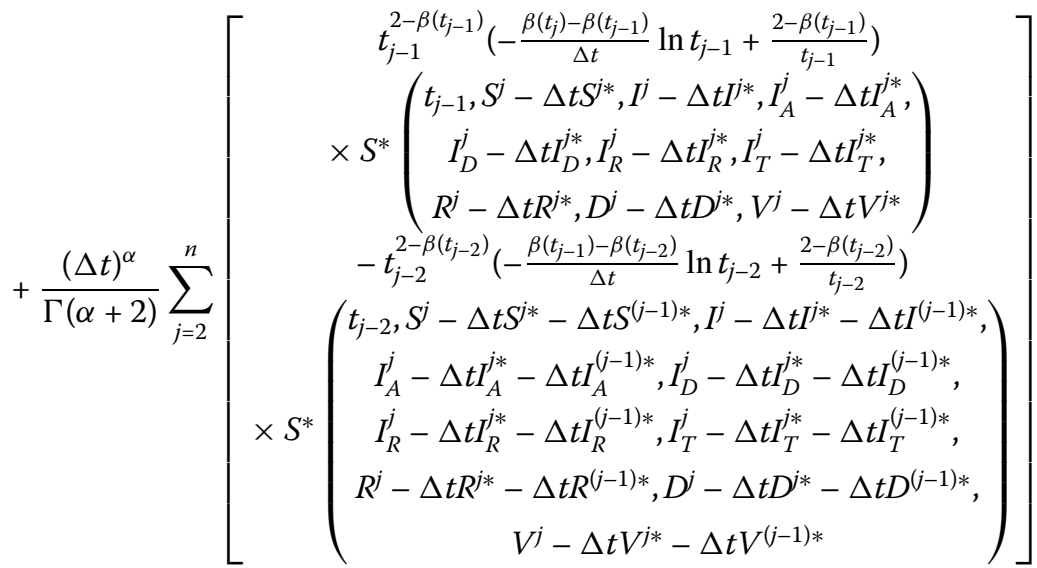


$+\frac{(\Delta t)^{\alpha}}{2 \Gamma(\alpha+3)} \sum_{j=2}^{n}\left[\begin{array}{c}t_{j}^{2-\beta\left(t_{j}\right)}\left(-\frac{\beta\left(t_{j+1}\right)-\beta\left(t_{j}\right)}{\Delta t} \ln t_{j}+\frac{2-\beta\left(t_{j}\right)}{t_{j}}\right) \\ \times S^{*}\left(t_{j}, S^{j}, I^{j}, I_{A}^{j}, I_{D}^{j}, I_{R}^{j}, I_{T}^{j}, R^{j}, D^{j}, V^{j}\right) \\ -2 t_{j-1}^{2-\beta\left(t_{j-1}\right)}\left(-\frac{\beta\left(t_{j}\right)-\beta\left(t_{j-1}\right)}{\Delta t} \ln t_{j-1}+\frac{2-\beta\left(t_{j-1}\right)}{t_{j-1}}\right) \\ \times S^{*}\left(\begin{array}{c}t_{j-1}, S^{j}-\Delta t S^{j *}, I^{j}-\Delta t I^{j *}, I_{A}^{j}-\Delta t I_{A}^{j *}, \\ I_{D}^{j}-\Delta t I_{D}^{j *}, I_{R}^{j}-\Delta t I_{R}^{* *}, I_{T}^{j}-\Delta t I_{T}^{j *}, \\ R^{j}-\Delta t R^{j *}, D^{j}-\Delta t D^{j *}, V^{j}-\Delta t V^{j *}\end{array}\right) \\ +t_{j-2}^{2-\beta\left(t_{j-2}\right)}\left(-\frac{\beta\left(t_{j-1}\right)-\beta\left(t_{j-2}\right)}{\Delta t} \ln t_{j-2}+\frac{2-\beta\left(t_{j-2}\right)}{t_{j-2}}\right) \\ t_{j-2}, S^{j}-\Delta t S^{j *}-\Delta t S^{(j-1) *}, I^{j}-\Delta t I^{*}-\Delta t I^{(j-1) *}, \\ I_{A}^{j}-\Delta t I_{A}^{j *}-\Delta t I_{A}^{(j-1) *}, I_{D}^{j}-\Delta t I_{D}^{j *}-\Delta t I_{D}^{(j-1) *}, \\ I_{R}^{j}-\Delta t I_{R}^{j *}-\Delta t I_{R}^{(j-1) *}, I_{T}^{j}-\Delta t I_{T}^{j *}-\Delta t I_{T}^{(j-1) *}, \\ R^{j}-\Delta t R^{R^{*}}-\Delta t R^{(j-1) *}, D^{j}-\Delta t D^{j *}-\Delta t D^{(j-1) *}, \\ V^{j}-\Delta t V^{j *}-\Delta t V^{(j-1) *}\end{array}\right)$

$\times \Delta$,

$I^{n+1}=\frac{(\Delta t)^{\alpha}}{\Gamma(\alpha+1)} \sum_{j=2}^{n} t_{j-2}^{2-\beta\left(t_{j-2}\right)}\left(-\frac{\beta\left(t_{j-1}\right)-\beta\left(t_{j-2}\right)}{\Delta t} \ln t_{j-2}+\frac{2-\beta\left(t_{j-2}\right)}{t_{j-2}}\right)$

$\times I^{*}\left(\begin{array}{c}t_{j-2}, S^{j}-\Delta t S^{j *}-\Delta t S^{(j-1) *}, I^{j}-\Delta t I^{j *}-\Delta t I^{(j-1) *}, \\ I_{A}^{j}-\Delta t I_{A}^{j *}-\Delta t I_{A}^{(j-1) *}, I_{D}^{j}-\Delta t I_{D}^{j *}-\Delta t I_{D}^{(j-1) *}, \\ I_{R}^{j}-\Delta t I_{R}^{j *}-\Delta t I_{R}^{(j-1) *}, I_{T}^{j}-\Delta t I_{T}^{j *}-\Delta t I_{T}^{(j-1) *}, \\ R^{j}-\Delta t R^{j *}-\Delta t R^{(j-1) *}, D^{j}-\Delta t D^{j *}-\Delta t D^{(j-1) *}, \\ V^{j}-\Delta t V^{j *}-\Delta t V^{(j-1) *}\end{array}\right) \times \Pi$

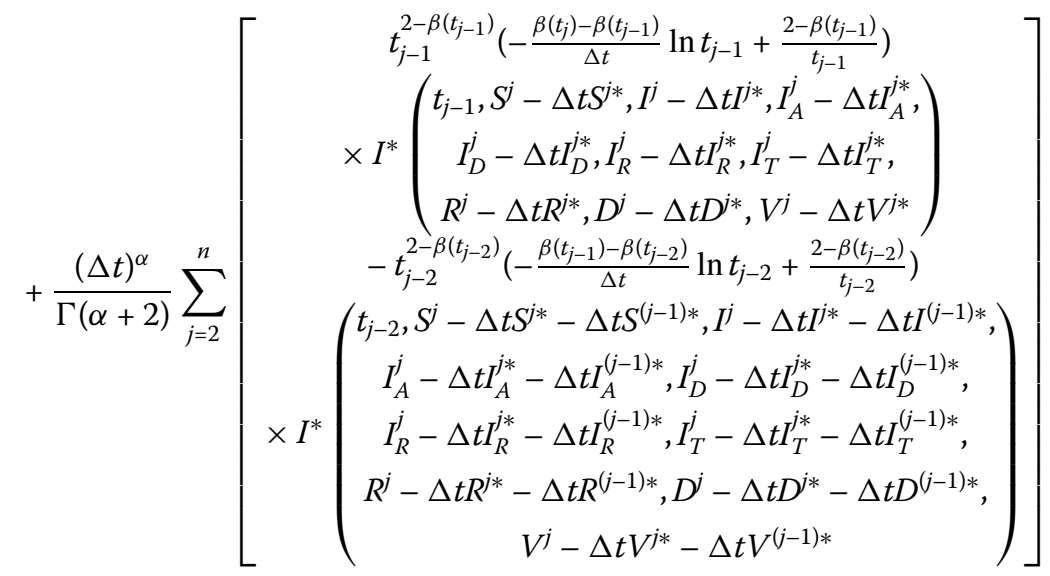

$\times \Sigma$ 


$$
+\frac{\alpha(\Delta t)^{\alpha}}{2 \Gamma(\alpha+3)} \sum_{j=2}^{n}\left[\begin{array}{c}
t_{j}^{2-\beta\left(t_{j}\right)}\left(-\frac{\beta\left(t_{j+1}\right)-\beta\left(t_{j}\right)}{\Delta t} \ln t_{j}+\frac{2-\beta\left(t_{j}\right)}{t_{j}}\right) \\
\times I^{*}\left(t_{j}, S^{j}, I^{j}, I_{A}^{j}, I_{D}^{j}, I_{R}^{j}, I_{T}^{j}, R^{j}, D^{j}, V^{j}\right) \\
-2 t_{j-1}^{2-\beta\left(t_{j-1}\right)}\left(-\frac{\beta\left(t_{j}\right)-\beta\left(t_{j-1}\right)}{\Delta t} \ln t_{j-1}+\frac{2-\beta\left(t_{j-1}\right)}{t_{j-1}}\right) \\
\times I^{*}\left(\begin{array}{c}
t_{j-1}, S^{j}-\Delta t S^{j *}, I^{j}-\Delta t I^{j *}, I_{A}^{j}-\Delta t I_{A}^{j *}, \\
I_{D}^{j}-\Delta t I_{D}^{j *}, I_{R}^{j}-\Delta t I_{R}^{j *}, I_{T}^{j}-\Delta t I_{T}^{j *}, \\
R^{j}-\Delta t R^{j *}, D^{j}-\Delta t D^{j *}, V^{j}-\Delta t V^{j *}
\end{array}\right) \\
+t_{j-2}^{2-\beta\left(t_{j-2}\right)}\left(-\frac{\beta\left(t_{j-1}\right)-\beta\left(t_{j-2}\right)}{\Delta t} \ln t_{j-2}+\frac{2-\beta\left(t_{j-2}\right)}{t_{j-2}}\right) \\
t_{j-2}, S^{j}-\Delta t S^{j *}-\Delta t S^{(j-1) *}, I^{j}-\Delta t I^{*}-\Delta t I^{(j-1) *}, \\
I_{A}^{j}-\Delta t I_{A}^{j *}-\Delta t I_{A}^{(j-1) *}, I_{D}^{j}-\Delta t I_{D}^{j *}-\Delta t I_{D}^{(j-1) *}, \\
I_{R}^{j}-\Delta t I_{R}^{j *}-\Delta t I_{R}^{(j-1) *}, I_{T}^{j}-\Delta t I_{T}^{j *}-\Delta t I_{T}^{(j-1) *}, \\
R^{j}-\Delta t R^{*}-\Delta t R^{(j-1) *}, D^{j}-\Delta t D^{j *}-\Delta t D^{(j-1) *}, \\
V^{j}-\Delta t V^{j *}-\Delta t V^{(j-1) *}
\end{array}\right)
$$

$\times \Delta$,

$$
\begin{aligned}
& I_{A}^{n+1}=\frac{(\Delta t)^{\alpha}}{\Gamma(\alpha+1)} \sum_{j=2}^{n} t_{j-2}^{2-\beta\left(t_{j-2}\right)}\left(-\frac{\beta\left(t_{j-1}\right)-\beta\left(t_{j-2}\right)}{\Delta t} \ln t_{j-2}+\frac{2-\beta\left(t_{j-2}\right)}{t_{j-2}}\right) \\
& \times I_{A}^{*}\left(\begin{array}{c}
t_{j-2}, S^{j}-\Delta t S^{j *}-\Delta t S^{(j-1) *}, I^{j}-\Delta t I^{j *}-\Delta t I^{(j-1) *}, \\
I_{A}^{j}-\Delta t I_{A}^{j *}-\Delta t I_{A}^{(j-1) *}, I_{D}^{j}-\Delta t I_{D}^{j *}-\Delta t I_{D}^{(j-1) *}, \\
I_{R}^{j}-\Delta t I_{R}^{j *}-\Delta t I_{R}^{(j-1) *}, I_{T}^{j}-\Delta t I_{T}^{j *}-\Delta t I_{T}^{(j-1) *}, \\
R^{j}-\Delta t R^{j *}-\Delta t R^{(j-1) *}, D^{j}-\Delta t D^{j *}-\Delta t D^{(j-1) *}, \\
V^{j}-\Delta t V^{j *}-\Delta t V^{(j-1) *}
\end{array}\right) \times \Pi
\end{aligned}
$$

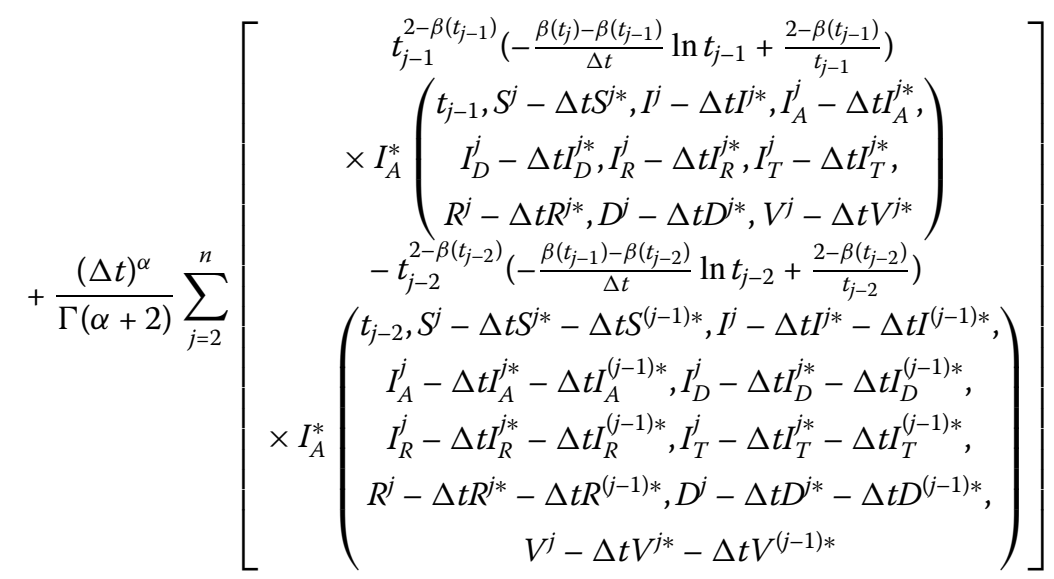




$$
+\frac{(\Delta t)^{\alpha}}{2 \Gamma(\alpha+3)} \sum_{j=2}^{n}\left[\begin{array}{c}
t_{j}^{2-\beta\left(t_{j}\right)}\left(-\frac{\beta\left(t_{j+1}\right)-\beta\left(t_{j}\right)}{\Delta t} \ln t_{j}+\frac{2-\beta\left(t_{j}\right)}{t_{j}}\right) \\
\times I_{A}^{*}\left(t_{j}, S^{j}, I^{j}, I_{A}^{j}, I_{D}^{j}, I_{R}^{j}, I_{T}^{j}, R^{j}, D^{j}, V^{j}\right) \\
-2 t_{j-1}^{2-\beta\left(t_{j-1}\right)}\left(-\frac{\beta\left(t_{j}\right)-\beta\left(t_{j-1}\right)}{\Delta t} \ln t_{j-1}+\frac{2-\beta\left(t_{j-1}\right)}{t_{j-1}}\right) \\
\times I_{A}^{*}\left(\begin{array}{c}
t_{j-1}, S^{j}-\Delta t S^{j *}, I^{j}-\Delta t I^{j *}, I_{A}^{j}-\Delta t I_{A}^{j *}, \\
I_{D}^{j}-\Delta t I_{D}^{j *}, I_{R}^{j}-\Delta t I_{R}^{* *}, I_{T}^{j}-\Delta t I_{T}^{j *}, \\
R^{j}-\Delta t R^{j *}, D^{j}-\Delta t D^{j *}, V^{j}-\Delta t V^{j *}
\end{array}\right) \\
+t_{j-2}^{2-\beta\left(t_{j-2}\right)}\left(-\frac{\beta\left(t_{j-1}\right)-\beta\left(t_{j-2}\right)}{\Delta t} \ln t_{j-2}+\frac{2-\beta\left(t_{j-2}\right)}{t_{j-2}}\right) \\
t_{j-2}, S^{j}-\Delta t S^{j *}-\Delta t S^{(j-1) *}, I^{j}-\Delta t I^{* *}-\Delta t I^{(j-1) *}, \\
I_{A}^{j}-\Delta t I_{A}^{j *}-\Delta t I_{A}^{(j-1) *}, I_{D}^{j}-\Delta t I_{D}^{j *}-\Delta t I_{D}^{(j-1) *}, \\
I_{R}^{j}-\Delta t I_{R}^{j *}-\Delta t I_{R}^{(j-1) *}, I_{T}^{j}-\Delta t I_{T}^{j *}-\Delta t I_{T}^{(j-1) *}, \\
R^{j}-\Delta t R^{j^{*}}-\Delta t R^{(j-1) *}, D^{j}-\Delta t D^{j *}-\Delta t D^{(j-1) *}, \\
V^{j}-\Delta t V^{j *}-\Delta t V^{(j-1) *}
\end{array}\right)
$$

$\times \Delta$,

$$
\begin{aligned}
& I_{D}^{n+1}=\frac{(\Delta t)^{\alpha}}{\Gamma(\alpha+1)} \sum_{j=2}^{n} t_{j-2}^{2-\beta\left(t_{j-2}\right)}\left(-\frac{\beta\left(t_{j-1}\right)-\beta\left(t_{j-2}\right)}{\Delta t} \ln t_{j-2}+\frac{2-\beta\left(t_{j-2}\right)}{t_{j-2}}\right) \\
& \times I_{D}^{*}\left(\begin{array}{c}
t_{j-2}, S^{j}-\Delta t S^{j *}-\Delta t S^{(j-1) *}, I^{j}-\Delta t I^{j *}-\Delta t I^{(j-1) *}, \\
I_{A}^{j}-\Delta t I_{A}^{j *}-\Delta t I_{A}^{(j-1) *}, I_{D}^{j}-\Delta t I_{D}^{j *}-\Delta t I_{D}^{(j-1) *}, \\
I_{R}^{j}-\Delta t I_{R}^{j *}-\Delta t I_{R}^{(j-1) *}, I_{T}^{j}-\Delta t I_{T}^{j *}-\Delta t I_{T}^{(j-1) *}, \\
R^{j}-\Delta t R^{j *}-\Delta t R^{(j-1) *}, D^{j}-\Delta t D^{j *}-\Delta t D^{(j-1) *}, \\
V^{j}-\Delta t V^{j *}-\Delta t V^{(j-1) *}
\end{array}\right) \times \Pi
\end{aligned}
$$

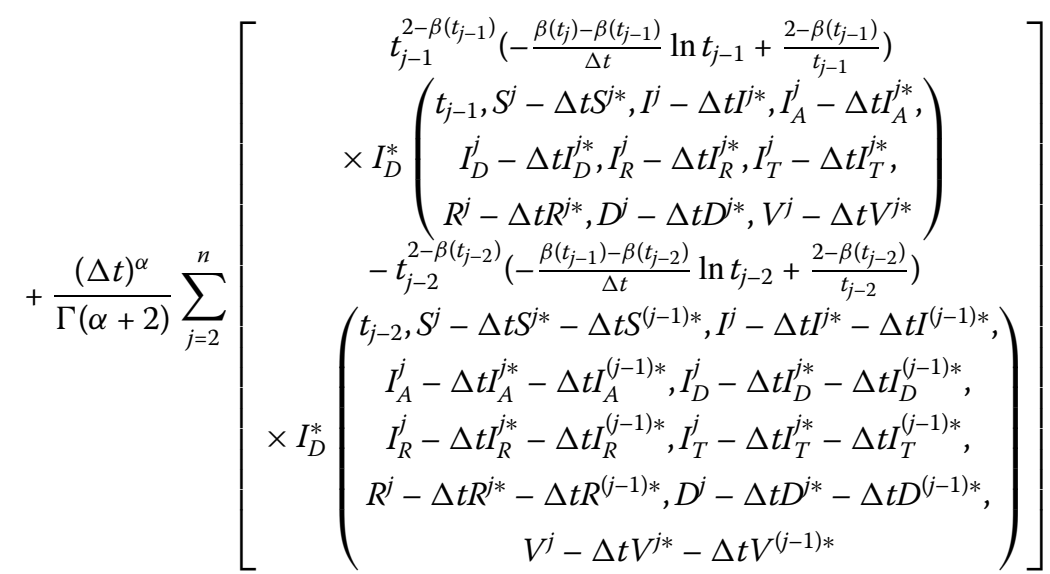




$$
+\frac{(\Delta t)^{\alpha}}{2 \Gamma(\alpha+3)} \sum_{j=2}^{n}\left[\begin{array}{c}
t_{j}^{2-\beta\left(t_{j}\right)}\left(-\frac{\beta\left(t_{j+1}\right)-\beta\left(t_{j}\right)}{\Delta t} \ln t_{j}+\frac{2-\beta\left(t_{j}\right)}{t_{j}}\right) \\
\times I_{D}^{*}\left(t_{j}, S^{j}, I^{j}, I_{A}^{j}, I_{D}^{j}, I_{R}^{j}, I_{T}^{j}, R^{j}, D^{j}, V^{j}\right) \\
-2 t_{j-1}^{2-\left(t_{j-1}\right)}\left(-\frac{\beta\left(t_{j}\right)-\beta\left(t_{j-1}\right)}{\Delta t} \ln t_{j-1}+\frac{2-\beta\left(t_{j-1}\right)}{t_{j-1}}\right) \\
\times I_{D}^{*}\left(\begin{array}{c}
t_{j-1}, S^{j}-\Delta t S^{j *}, I^{j}-\Delta t I^{j *}, I_{A}^{j}-\Delta t I_{A}^{j *}, \\
I_{D}^{j}-\Delta t I_{D}^{j *}, I_{R}^{j}-\Delta t I_{R}^{j *}, I_{T}^{j}-\Delta t I_{T}^{j *}, \\
R^{j}-\Delta t R^{j *}, D^{j}-\Delta t D^{j *}, V^{j}-\Delta t V^{j *}
\end{array}\right) \\
+t_{j-2}^{2-\beta\left(t_{j-2}\right)}\left(-\frac{\beta\left(t_{j-1}\right)-\beta\left(t_{j-2}\right)}{\Delta t} \ln t_{j-2}+\frac{2-\beta\left(t_{j-2}\right)}{t_{j-2}}\right) \\
t_{j-2}, S^{j}-\Delta t S^{j *}-\Delta t S^{(j-1) *}, I^{j}-\Delta t I^{j *}-\Delta t I^{(j-1) *}, \\
I_{A}^{j}-\Delta t I_{A}^{j *}-\Delta t I_{A}^{(j-1) *}, I_{D}^{j}-\Delta t I_{D}^{j *}-\Delta t I_{D}^{(j-1) *}, \\
I_{R}^{j}-\Delta t I_{R}^{j *}-\Delta t I_{R}^{(j-1) *}, I_{T}^{j}-\Delta t I_{T}^{j *}-\Delta t I_{T}^{(j-1) *}, \\
R^{j}-\Delta t R^{*}-\Delta t R^{(j-1) *}, D^{j}-\Delta t D^{j *}-\Delta t D^{(j-1) *}, \\
V^{j}-\Delta t V^{j *}-\Delta t V^{(j-1) *}
\end{array}\right)
$$

$\times \Delta$,

$$
\begin{aligned}
& I_{R}^{n+1}=\frac{(\Delta t)^{\alpha}}{\Gamma(\alpha+1)} \sum_{j=2}^{n} t_{j-2}^{2-\beta\left(t_{j-2}\right)}\left(-\frac{\beta\left(t_{j-1}\right)-\beta\left(t_{j-2}\right)}{\Delta t} \ln t_{j-2}+\frac{2-\beta\left(t_{j-2}\right)}{t_{j-2}}\right) \\
& \times I_{R}^{*}\left(\begin{array}{c}
t_{j-2}, S^{j}-\Delta t S^{j *}-\Delta t S^{(j-1) *}, I^{j}-\Delta t I^{j *}-\Delta t I^{(j-1) *}, \\
I_{A}^{j}-\Delta t I_{A}^{j *}-\Delta t I_{A}^{(j-1) *}, I_{D}^{j}-\Delta t I_{D}^{j *}-\Delta t I_{D}^{(j-1) *}, \\
I_{R}^{j}-\Delta t I_{R}^{j *}-\Delta t I_{R}^{(j-1) *}, I_{T}^{j}-\Delta t I_{T}^{j *}-\Delta t I_{T}^{(j-1) *}, \\
R^{j}-\Delta t R^{j *}-\Delta t R^{(j-1) *}, D^{j}-\Delta t D^{j *}-\Delta t D^{(j-1) *}, \\
V^{j}-\Delta t V^{j *}-\Delta t V^{(j-1) *}
\end{array}\right) \times \Pi
\end{aligned}
$$

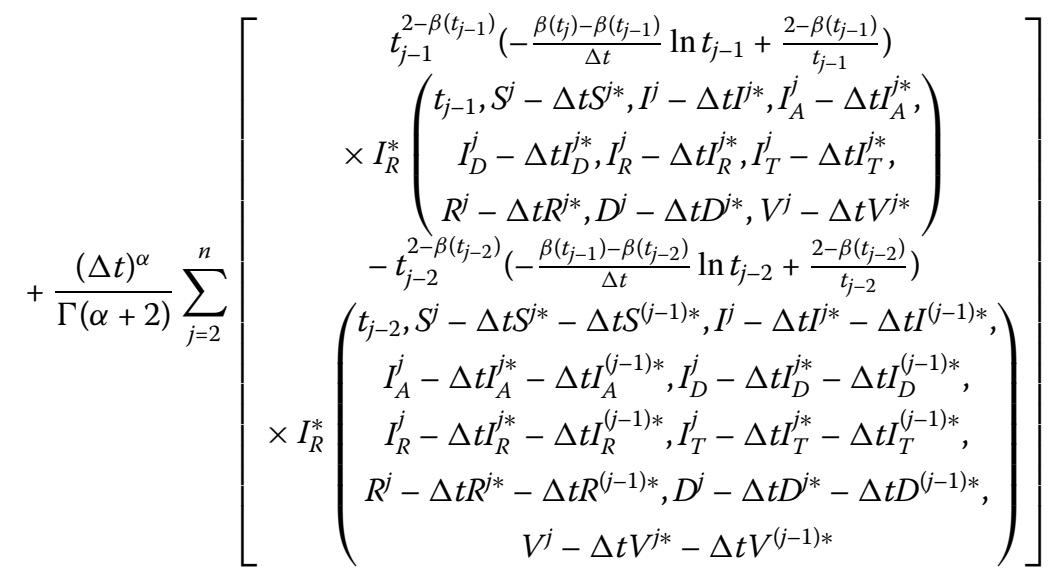




$$
+\frac{(\Delta t)^{\alpha}}{2 \Gamma(\alpha+3)} \sum_{j=2}^{n}\left[\begin{array}{c}
t_{j}^{2-\beta\left(t_{j}\right)}\left(-\frac{\beta\left(t_{j+1}\right)-\beta\left(t_{j}\right)}{\Delta t} \ln t_{j}+\frac{2-\beta\left(t_{j}\right)}{t_{j}}\right) \\
\times I_{R}^{*}\left(t_{j}, S^{j}, I^{j}, I_{A}^{j}, I_{D}^{j}, I_{R}^{j}, I_{T}^{j}, R^{j}, D^{j}, V^{j}\right) \\
-2 t_{j-1}^{2-\beta\left(t_{j-1}\right)}\left(-\frac{\beta\left(t_{j}\right)-\beta\left(t_{j-1}\right)}{\Delta t} \ln t_{j-1}+\frac{2-\beta\left(t_{j-1}\right)}{t_{j-1}}\right) \\
\times I_{R}^{*}\left(\begin{array}{c}
t_{j-1}, S^{j}-\Delta t S^{j *}, I^{j}-\Delta t I^{j *}, I_{A}^{j}-\Delta t I_{A}^{j *}, \\
I_{D}^{j}-\Delta t I_{D}^{j *}, I_{R}^{j}-\Delta t I_{R}^{j *}, I_{T}^{j}-\Delta t I_{T}^{j *}, \\
R^{j}-\Delta t R^{j *}, D^{j}-\Delta t D^{j *}, V^{j}-\Delta t V^{j *}
\end{array}\right) \\
+t_{j-2}^{2-\beta\left(t_{j-2}\right)}\left(-\frac{\beta\left(t_{j-1}\right)-\beta\left(t_{j-2}\right)}{\Delta t} \ln t_{j-2}+\frac{2-\beta\left(t_{j-2}\right)}{t_{j-2}}\right) \\
t_{j-2}, S^{j}-\Delta t S^{j *}-\Delta t S^{(j-1) *}, I^{j}-\Delta t I^{j *}-\Delta t I^{(j-1) *}, \\
I_{A}^{j}-\Delta t I_{A}^{j *}-\Delta t I_{A}^{(j-1) *}, I_{D}^{j}-\Delta t I_{D}^{j *}-\Delta t I_{D}^{(j-1) *}, \\
I_{R}^{j}-\Delta t I_{R}^{j *}-\Delta t I_{R}^{(j-1) *}, I_{T}^{j}-\Delta t I_{T}^{j *}-\Delta t I_{T}^{(j-1) *}, \\
R^{j}-\Delta t R^{*}-\Delta t R^{(j-1) *}, D^{j}-\Delta t D^{j *}-\Delta t D^{(j-1) *}, \\
V^{j}-\Delta t V^{j *}-\Delta t V^{(j-1) *}
\end{array}\right)
$$

$\times \Delta$,

$$
\begin{aligned}
& I_{T}^{n+1}=\frac{(\Delta t)^{\alpha}}{\Gamma(\alpha+1)} \sum_{j=2}^{n} t_{j-2}^{2-\beta\left(t_{j-2}\right)}\left(-\frac{\beta\left(t_{j-1}\right)-\beta\left(t_{j-2}\right)}{\Delta t} \ln t_{j-2}+\frac{2-\beta\left(t_{j-2}\right)}{t_{j-2}}\right) \\
& \times I_{T}^{*}\left(\begin{array}{c}
t_{j-2}, S^{j}-\Delta t S^{j *}-\Delta t S^{(j-1) *}, I^{j}-\Delta t I^{j *}-\Delta t I^{(j-1) *}, \\
I_{A}^{j}-\Delta t I_{A}^{j *}-\Delta t I_{A}^{(j-1) *}, I_{D}^{j}-\Delta t I_{D}^{j *}-\Delta t I_{D}^{(j-1) *}, \\
I_{R}^{j}-\Delta t I_{R}^{j *}-\Delta t I_{R}^{(j-1) *}, I_{T}^{j}-\Delta t I_{T}^{j *}-\Delta t I_{T}^{(j-1) *}, \\
R^{j}-\Delta t R^{j *}-\Delta t R^{(j-1) *}, D^{j}-\Delta t D^{j *}-\Delta t D^{(j-1) *}, \\
V^{j}-\Delta t V^{j *}-\Delta t V^{(j-1) *}
\end{array}\right) \times \Pi
\end{aligned}
$$

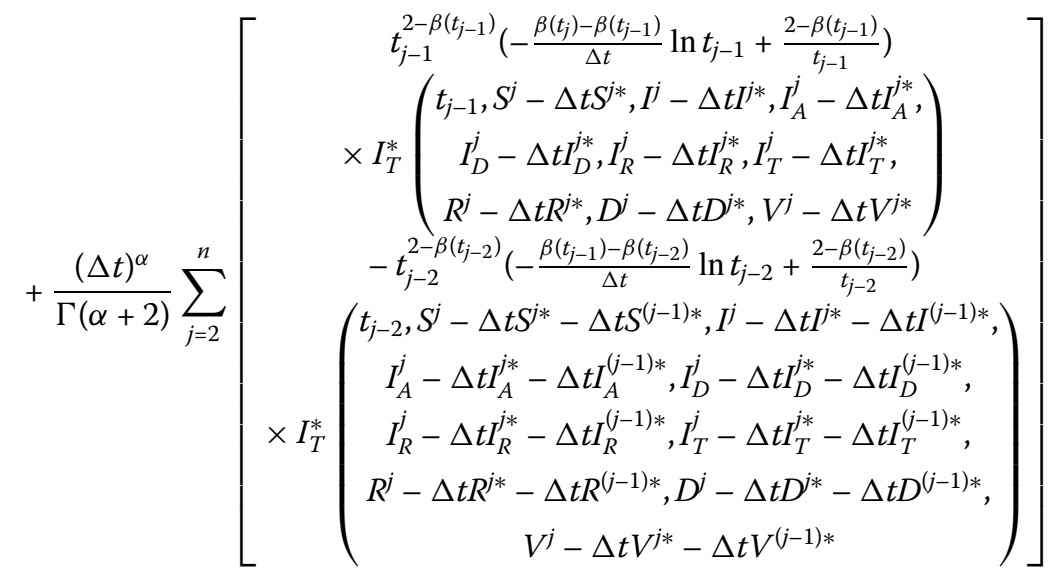




$$
+\frac{(\Delta t)^{\alpha}}{2 \Gamma(\alpha+3)} \sum_{j=2}^{n}\left[\begin{array}{c}
t_{j}^{2-\beta\left(t_{j}\right)}\left(-\frac{\beta\left(t_{j+1}\right)-\beta\left(t_{j}\right)}{\Delta t} \ln t_{j}+\frac{2-\beta\left(t_{j}\right)}{t_{j}}\right) \\
\times I_{T}^{*}\left(t_{j}, S^{j}, I^{j}, I_{A}^{j}, I_{D}^{j}, I_{R}^{j}, I_{T}^{j}, R^{j}, D^{j}, V^{j}\right) \\
-2 t_{j-1}^{2-\beta\left(t_{j-1}\right)}\left(-\frac{\beta\left(t_{j}\right)-\beta\left(t_{j-1}\right)}{\Delta t} \ln t_{j-1}+\frac{2-\beta\left(t_{j-1}\right)}{t_{j-1}}\right) \\
\times I_{T}^{*}\left(\begin{array}{c}
t_{j-1}, S^{j}-\Delta t S^{j *}, I^{j}-\Delta t I^{j *}, I_{A}^{j}-\Delta t I_{A}^{j *}, \\
I_{D}^{j}-\Delta t I_{D}^{j *}, I_{R}^{j}-\Delta t I_{R}^{j *}, I_{T}^{j}-\Delta t I_{T}^{j *}, \\
R^{j}-\Delta t R^{j *}, D^{j}-\Delta t D^{j *}, V^{j}-\Delta t V^{j *}
\end{array}\right) \\
+t_{j-2}^{2-\beta\left(t_{j-2}\right)}\left(-\frac{\beta\left(t_{j-1}\right)-\beta\left(t_{j-2}\right)}{\Delta t} \ln t_{j-2}+\frac{2-\beta\left(t_{j-2}\right)}{t_{j-2}}\right) \\
t_{j-2}, S^{j}-\Delta t S^{j *}-\Delta t S^{(j-1) *}, I^{j}-\Delta t I^{j *}-\Delta t I^{(j-1) *}, \\
I_{A}^{j}-\Delta t I_{A}^{j *}-\Delta t I_{A}^{(j-1) *}, I_{D}^{j}-\Delta t I_{D}^{j *}-\Delta t I_{D}^{(j-1) *}, \\
I_{R}^{j}-\Delta t I_{R}^{j *}-\Delta t I_{R}^{(j-1) *}, I_{T}^{j}-\Delta t I_{T}^{j *}-\Delta t I_{T}^{(j-1) *}, \\
R^{j}-\Delta t R^{j *}-\Delta t R^{(j-1) *}, D^{j}-\Delta t D^{j *}-\Delta t D^{(j-1) *}, \\
V^{j}-\Delta t V^{j *}-\Delta t V^{(j-1) *}
\end{array}\right]
$$

$\times \Delta$,

$$
\begin{aligned}
& R^{n+1}=\frac{(\Delta t)^{\alpha}}{\Gamma(\alpha+1)} \sum_{j=2}^{n} t_{j-2}^{2-\beta\left(t_{j-2}\right)}\left(-\frac{\beta\left(t_{j-1}\right)-\beta\left(t_{j-2}\right)}{\Delta t} \ln t_{j-2}+\frac{2-\beta\left(t_{j-2}\right)}{t_{j-2}}\right) \\
& \times R^{*}\left(\begin{array}{c}
t_{j-2}, S^{j}-\Delta t S^{j *}-\Delta t S^{(j-1) *}, I^{j}-\Delta t I^{j *}-\Delta t I^{(j-1) *}, \\
I_{A}^{j}-\Delta t I_{A}^{j *}-\Delta t I_{A}^{(j-1) *}, I_{D}^{j}-\Delta t I_{D}^{j *}-\Delta t I_{D}^{(j-1) *}, \\
I_{R}^{j}-\Delta t I_{R}^{j *}-\Delta t I_{R}^{(j-1) *}, I_{T}^{j}-\Delta t I_{T}^{j *}-\Delta t I_{T}^{(j-1) *}, \\
R^{j}-\Delta t R^{j *}-\Delta t R^{(j-1) *}, D^{j}-\Delta t D^{j *}-\Delta t D^{(j-1) *}, \\
V^{j}-\Delta t V^{j *}-\Delta t V^{(j-1) *}
\end{array}\right) \times \Pi
\end{aligned}
$$

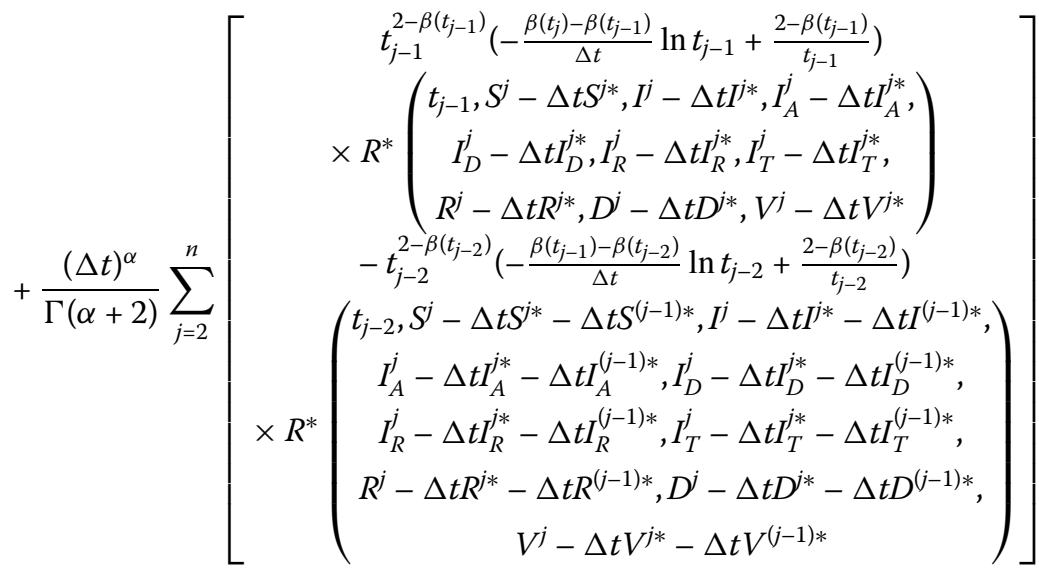

$\times \Sigma$ 


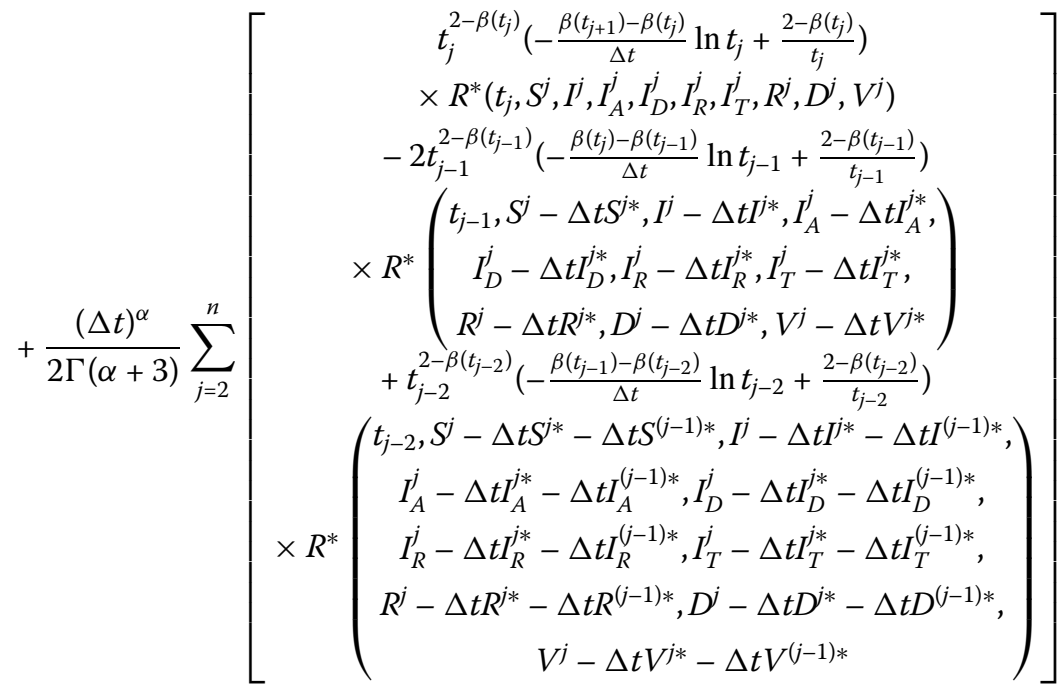

$\times \Delta$

$$
D^{n+1}=\frac{(\Delta t)^{\alpha}}{\Gamma(\alpha+1)} \sum_{j=2}^{n} t_{j-2}^{2-\beta\left(t_{j-2}\right)}\left(-\frac{\beta\left(t_{j-1}\right)-\beta\left(t_{j-2}\right)}{\Delta t} \ln t_{j-2}+\frac{2-\beta\left(t_{j-2}\right)}{t_{j-2}}\right)
$$$$
\times D^{*}\left(\begin{array}{c}
t_{j-2}, S^{j}-\Delta t S^{j *}-\Delta t S^{(j-1) *}, I^{j}-\Delta t I^{j *}-\Delta t I^{(j-1) *}, \\
I_{A}^{j}-\Delta t I_{A}^{j *}-\Delta t I_{A}^{(j-1) *}, I_{D}^{j}-\Delta t I_{D}^{j *}-\Delta t I_{D}^{(j-1) *}, \\
I_{R}^{j}-\Delta t I_{R}^{j *}-\Delta t I_{R}^{(j-1) *}, I_{T}^{j}-\Delta t I_{T}^{j *}-\Delta t I_{T}^{(j-1) *}, \\
R^{j}-\Delta t R^{j *}-\Delta t R^{(j-1) *}, D^{j}-\Delta t D^{j *}-\Delta t D^{(j-1) *}, \\
V^{j}-\Delta t V^{j *}-\Delta t V^{(j-1) *}
\end{array}\right) \times \Pi
$$

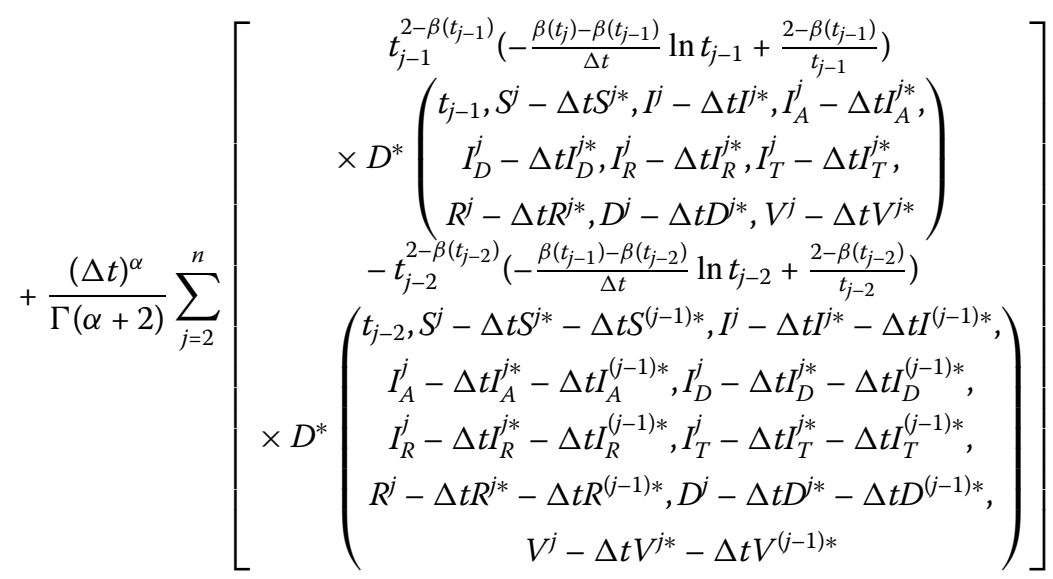

$\times \Sigma$ 
$\left.+\frac{(\Delta t)^{\alpha}}{2 \Gamma(\alpha+3)} \sum_{j=2}^{n}\left[\begin{array}{c}t_{j}^{2-\beta\left(t_{j}\right)}\left(-\frac{\beta\left(t_{j+1}\right)-\beta\left(t_{j}\right)}{\Delta t} \ln t_{j}+\frac{2-\beta\left(t_{j}\right)}{t_{j}}\right) \\ \times D^{*}\left(t_{j}, S^{j}, I^{j}, I_{A}^{j}, I_{D}^{j}, I_{R}^{j}, I_{T}^{j}, R^{j}, D^{j}, V^{j}\right) \\ -2 t_{j-1}^{2-\beta\left(t_{j-1}\right)}\left(-\frac{\beta\left(t_{j}\right)-\beta\left(t_{j-1}\right)}{\Delta t} \ln t_{j-1}+\frac{2-\beta\left(t_{j-1}\right)}{t_{j-1}}\right) \\ \times D^{*}\left(\begin{array}{c}t_{j-1}, S^{j}-\Delta t S^{*}, I^{j}-\Delta t I^{j *}, I_{A}^{j}-\Delta t I_{A}^{* *}, \\ I_{D}^{j}-\Delta t I_{D}^{j *}, I_{R}^{j}-\Delta t I_{R}^{j *}, I_{T}^{j}-\Delta t I_{T}^{j *}, \\ R^{j}-\Delta t R^{j *}, D^{j}-\Delta t D^{j *}, V^{j}-\Delta t V^{j *}\end{array}\right) \\ +t_{j-2}^{2-\beta\left(t_{j-2}\right)}\left(-\frac{\beta\left(t_{j-1}\right)-\beta\left(t_{j-2}\right)}{\Delta t} \ln t_{j-2}+\frac{2-\beta\left(t_{j-2}\right)}{t_{j-2}}\right) \\ t_{j-2}, S^{j}-\Delta t S^{j *}-\Delta t S^{(j-1) *}, I^{j}-\Delta t I^{j *}-\Delta t I^{(j-1) *}, \\ I_{A}^{j}-\Delta t I_{A}^{j *}-\Delta t I_{A}^{(j-1) *}, I_{D}^{j}-\Delta t I_{D}^{j *}-\Delta t I_{D}^{(j-1) *}, \\ I_{R}^{j}-\Delta t I_{R}^{j *}-\Delta t I_{R}^{(j-1) *}, I_{T}^{j}-\Delta t I_{T}^{j *}-\Delta t I_{T}^{(j-1) *}, \\ R^{j}-\Delta t R^{j *}-\Delta t R^{(j-1) *}, D^{j}-\Delta t D^{j *}-\Delta t D^{(j-1) *}, \\ V^{j}-\Delta t V^{j *}-\Delta t V^{(j-1) *}\end{array}\right)\right]$

$\times \Delta$,

$$
V^{n+1}=\frac{(\Delta t)^{\alpha}}{\Gamma(\alpha+1)} \sum_{j=2}^{n} t_{j-2}^{2-\beta\left(t_{j-2}\right)}\left(-\frac{\beta\left(t_{j-1}\right)-\beta\left(t_{j-2}\right)}{\Delta t} \ln t_{j-2}+\frac{2-\beta\left(t_{j-2}\right)}{t_{j-2}}\right)
$$$$
\times V^{*}\left(\begin{array}{c}
t_{j-2}, S^{j}-\Delta t S^{j *}-\Delta t S^{(j-1) *}, I^{j}-\Delta t I^{j *}-\Delta t I^{(j-1) *}, \\
I_{A}^{j}-\Delta t I_{A}^{j *}-\Delta t I_{A}^{(j-1) *}, I_{D}^{j}-\Delta t I_{D}^{j *}-\Delta t I_{D}^{(j-1) *}, \\
I_{R}^{j}-\Delta t I_{R}^{j *}-\Delta t I_{R}^{(j-1) *}, I_{T}^{j}-\Delta t I_{T}^{j *}-\Delta t I_{T}^{(j-1) *}, \\
R^{j}-\Delta t R^{j *}-\Delta t R^{(j-1) *}, D^{j}-\Delta t D^{j *}-\Delta t D^{(j-1) *}, \\
V^{j}-\Delta t V^{j *}-\Delta t V^{(j-1) *}
\end{array}\right) \times \Pi
$$$$
+\frac{(\Delta t)^{\alpha}}{\Gamma(\alpha+2)} \sum_{j=2}^{n}\left[\begin{array}{c}
t_{j-1}^{2-\beta\left(t_{j-1}\right)}\left(-\frac{\beta\left(t_{j}\right)-\beta\left(t_{j-1}\right)}{\Delta t} \ln t_{j-1}+\frac{2-\beta\left(t_{j-1}\right)}{t_{j-1}}\right) \\
t_{j-1}, S^{j}-\Delta t S^{j *}, I^{j}-\Delta t I^{j *}, I_{A}^{j}-\Delta t I_{A}^{j *}, \\
I_{D}^{j}-\Delta t I_{D}^{j *}, I_{R}^{j}-\Delta t I_{R}^{j *}, I_{T}^{j}-\Delta t I_{T}^{j *}, \\
R^{j}-\Delta t R^{j *}, D^{j}-\Delta t D^{j *}, V^{j}-\Delta t V^{j *}
\end{array}\right)
$$

$\times \Sigma$ 


$$
+\frac{(\Delta t)^{\alpha}}{2 \Gamma(\alpha+3)} \sum_{j=2}^{n}\left[\begin{array}{c}
t_{j}^{2-\beta\left(t_{j}\right)}\left(-\frac{\beta\left(t_{j+1}\right)-\beta\left(t_{j}\right)}{\Delta t} \ln t_{j}+\frac{2-\beta\left(t_{j}\right)}{t_{j}}\right) \\
\times V^{*}\left(t_{j}, S^{j}, I^{j}, I_{A}^{j}, I_{D}^{j}, I_{R}^{j}, I_{T}^{j}, R^{j}, D^{j}, V^{j}\right) \\
-2 t_{j-1}^{2-\beta\left(t_{j-1}\right)}\left(-\frac{\beta\left(t_{j}\right)-\beta\left(t_{j-1}\right)}{\Delta t} \ln t_{j-1}+\frac{2-\beta\left(t_{j-1}\right)}{t_{j-1}}\right) \\
\times V^{*}\left(\begin{array}{c}
t_{j-1}, S^{j}-\Delta t S^{j *}, I^{j}-\Delta t I^{j *}, I_{A}^{j}-\Delta t I_{A}^{j *}, \\
I_{D}^{j}-\Delta t I_{D}^{j *}, I_{R}^{j}-\Delta t I_{R}^{j *}, I_{T}^{j}-\Delta t I_{T}^{j *}, \\
R^{j}-\Delta t R^{j *}, D^{j}-\Delta t D^{j *}, V^{j}-\Delta t V^{j *}
\end{array}\right) \\
+t_{j-2}^{2-\beta\left(t_{j-2}\right)}\left(-\frac{\beta\left(t_{j-1}\right)-\beta\left(t_{j-2}\right)}{\Delta t} \ln t_{j-2}+\frac{2-\beta\left(t_{j-2}\right)}{t_{j-2}}\right) \\
t_{j-2}, S^{j}-\Delta t S^{j *}-\Delta t S^{(j-1) *}, I^{j}-\Delta t I^{*}-\Delta t I^{(j-1) *}, \\
I_{A}^{j}-\Delta t I_{A}^{j *}-\Delta t I_{A}^{(j-1) *}, I_{D}^{j}-\Delta t I_{D}^{j *}-\Delta t I_{D}^{(j-1) *}, \\
I_{R}^{j}-\Delta t I_{R}^{j *}-\Delta t I_{R}^{(j-1) *}, I_{T}^{j}-\Delta t I_{T}^{j *}-\Delta t I_{T}^{(j-1) *}, \\
R^{j}-\Delta t R^{j *}-\Delta t R^{(j-1) *}, D^{j}-\Delta t D^{j *}-\Delta t D^{(j-1) *}, \\
V^{j}-\Delta t V^{j *}-\Delta t V^{(j-1) *}
\end{array}\right)
$$

$\times \Delta$.

\section{Numerical simulation}

In this section, using the numerical solutions obtained in the previous section, we present a numerical method for all cases. The numerical simulations are depicted for different values of fractional order and fractal dimension as presented in Figs. 26-37.

$$
\begin{aligned}
& { }_{0}^{F F M} D_{t}^{\alpha, \beta} S=\Lambda-\left(\delta(t)\left(\alpha I^{*}+w \beta I_{D}^{*}+\gamma w I_{A}^{*}+w \delta_{1} I_{R}^{*}+w \delta_{2} I_{T}^{*}\right)+\gamma_{1}+\mu_{1}\right) S, \\
& { }_{0}^{F F M} D_{t}^{\alpha, \beta} I=\left(\delta(t)\left(\alpha I^{*}+w \beta I_{D}^{*}+\gamma w I_{A}^{*}+w \delta_{1} I_{R}^{*}+w \delta_{2} I_{T}^{*}\right)\right) S-\left(\varepsilon+\xi+\lambda+\mu_{1}\right) I, \\
& { }_{0}^{F F M} D_{t}^{\alpha, \beta} I_{A}=\xi I-\left(\theta+\mu+\chi+\mu_{1}\right) I_{A}, \\
& { }_{0}^{F F M} D_{t}^{\alpha, \beta} I_{D}=\varepsilon I-\left(\eta+\varphi+\mu_{1}\right) I_{D},
\end{aligned}
$$

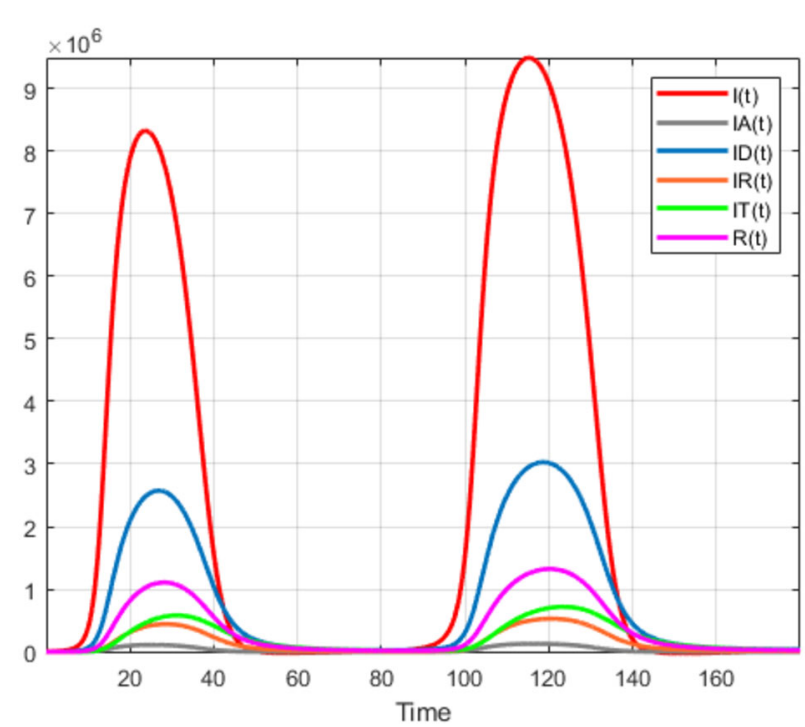

Figure 26 Numerical visualization of COVID-19 model for $\alpha=0.76$ 


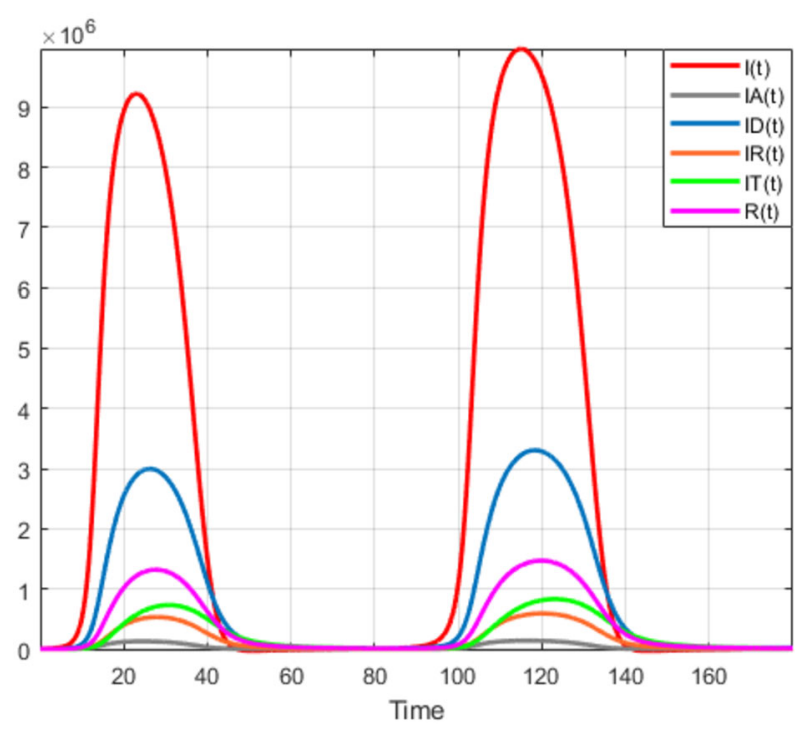

Figure 27 Numerical visualization of COVID-19 model for $\alpha=0.85$

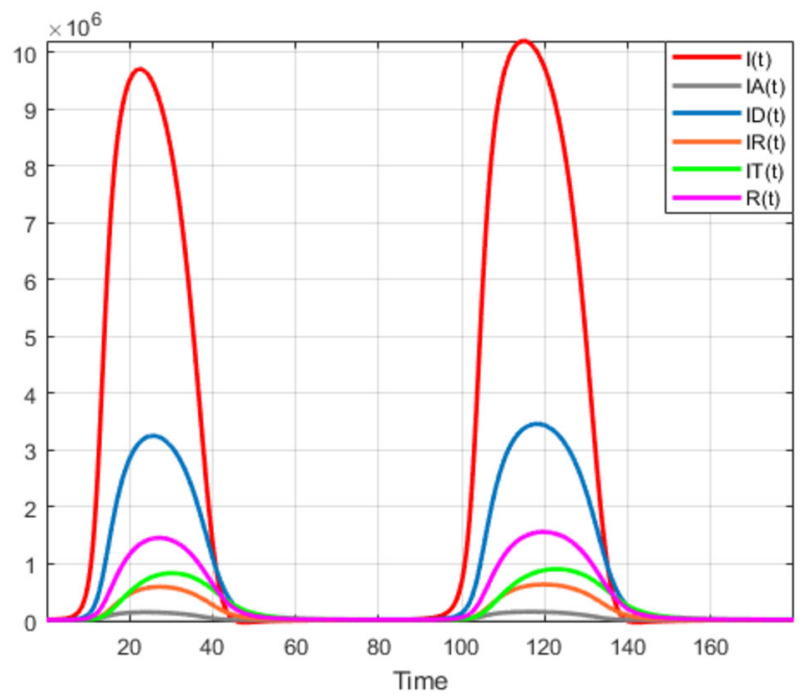

Figure 28 Numerical visualization of COVID-19 model for $\alpha=0.91$

$$
\begin{aligned}
& { }_{0}^{F F M} D_{t}^{\alpha, \beta} I_{R}=\eta I_{D}+\theta I_{A}-\left(v+\xi+\mu_{1}\right) I_{R}, \\
& { }_{0}^{F F M} D_{t}^{\alpha, \tau} I_{T}=\mu I_{A}+v I_{R}-\left(\sigma+\tau+\mu_{1}\right) I_{T}, \\
& { }_{0}^{F F M} D_{t}^{\alpha, \tau} R=\lambda I+\varphi I_{D}+\chi I_{A}+\xi I_{R}+\sigma I_{T}-\left(\Phi+\mu_{1}\right) R, \\
& { }_{0}^{F F M} D_{t}^{\alpha, \tau} D=\tau I_{T}, \\
& { }_{0}^{F F M} D_{t}^{\alpha, \tau} V=\gamma_{1} S+\Phi R-\mu_{1} V,
\end{aligned}
$$




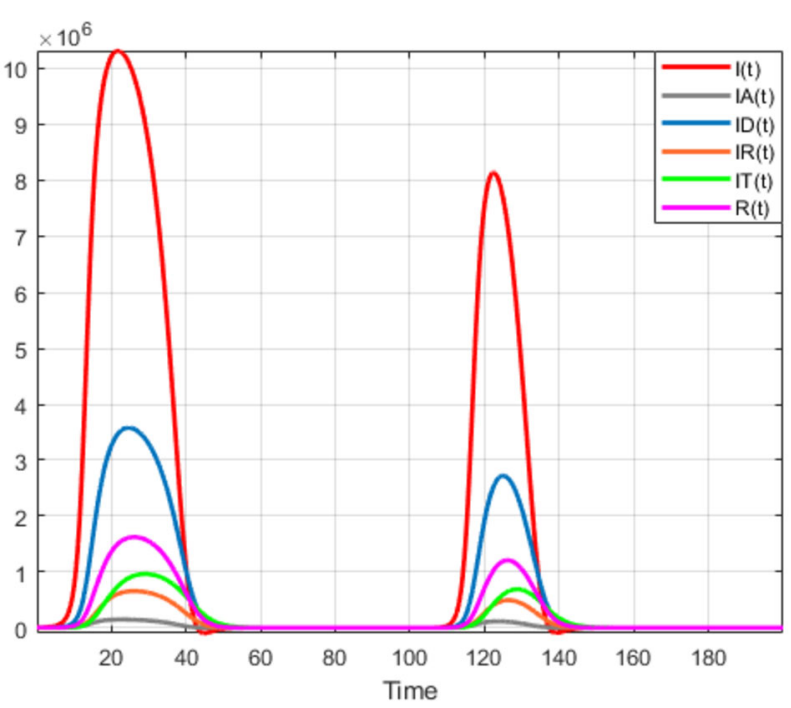

Figure 29 Numerical visualization of COVID-19 model for $\alpha=0.76$

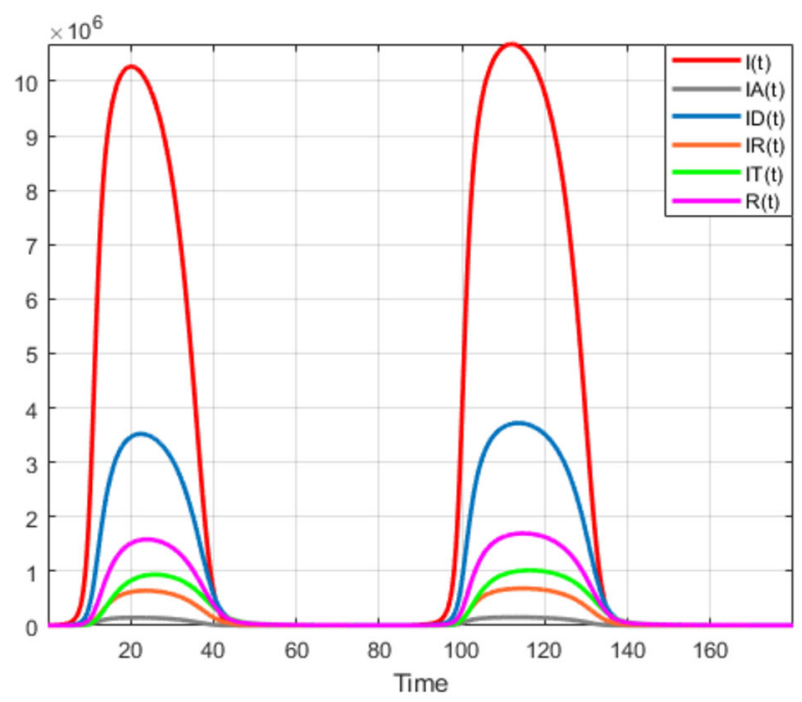

Figure 30 Numerical visualization of COVID-19 model for $\alpha=0.90, \beta=0.85$

where

$$
\delta(t)=\left\{\begin{array}{c}
d_{0}\left(1-a_{n}\right) \cos \left(-b \frac{t-t_{0}}{T}\right), 0<t<t_{0} \\
d_{0}, t_{0}<t<t_{1} \\
d_{1}\left(1-a_{r}\right) \cos \left(-b \frac{t-t_{1}}{T}\right), t_{1}<t<t_{2} \\
d_{1}, t_{2}<t<t_{3} \\
d_{2}\left(1-a_{t}\right) \cos \left(-b \frac{t-t_{2}}{T}\right), t>t_{3}
\end{array}\right\} .
$$




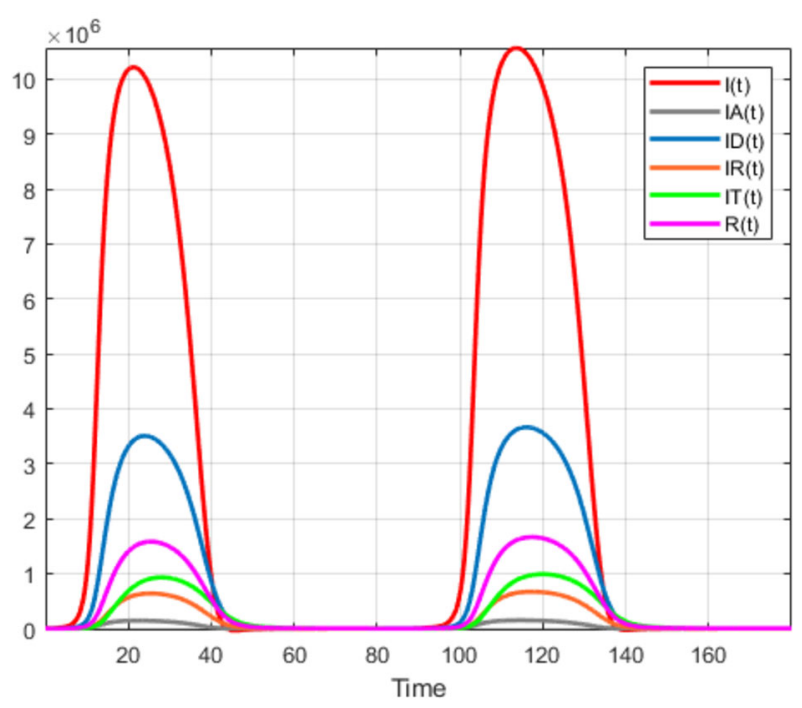

Figure 31 Numerical visualization of COVID-19 model for $\alpha=0.95, \beta=0.95$

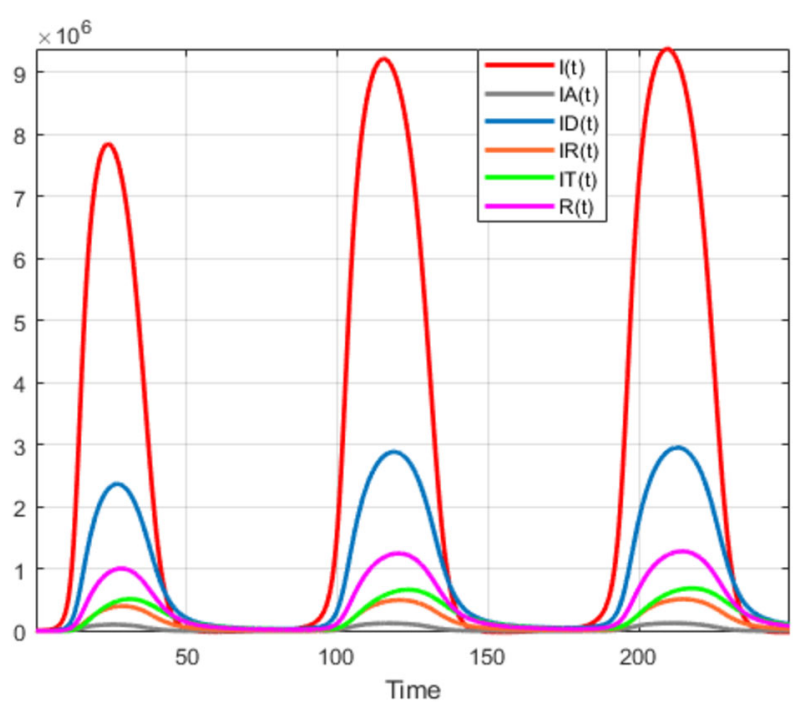

Figure 32 Numerical visualization of COVID-19 model for $\alpha=0.72$

Also, the initial conditions are

$$
\begin{aligned}
& S(0)=800,000, \quad I(0)=3, \quad I_{A}(0)=0, \quad I_{D}(0)=0, \quad I_{R}(0)=0, \\
& I_{T}(0)=0, \quad R(0)=0, \quad D(0)=0, \quad V(0)=0 .
\end{aligned}
$$

Also, the parameters are chosen as follows:

$$
\begin{aligned}
& \Lambda=810,000, \quad \eta=0.12, \quad \chi=0.15, \quad \quad \quad \quad \quad \quad \quad \gamma_{1}=0.4, \quad \mu_{1}=0.3, \quad \varepsilon=0.161, \quad \gamma=0.09, \quad \tau=0.0199, \\
& \beta=0.75, \quad \quad \lambda=0.0345, \quad \varphi=0.0345, \quad \delta_{1}=0.5, \quad \xi=0.015,
\end{aligned}
$$




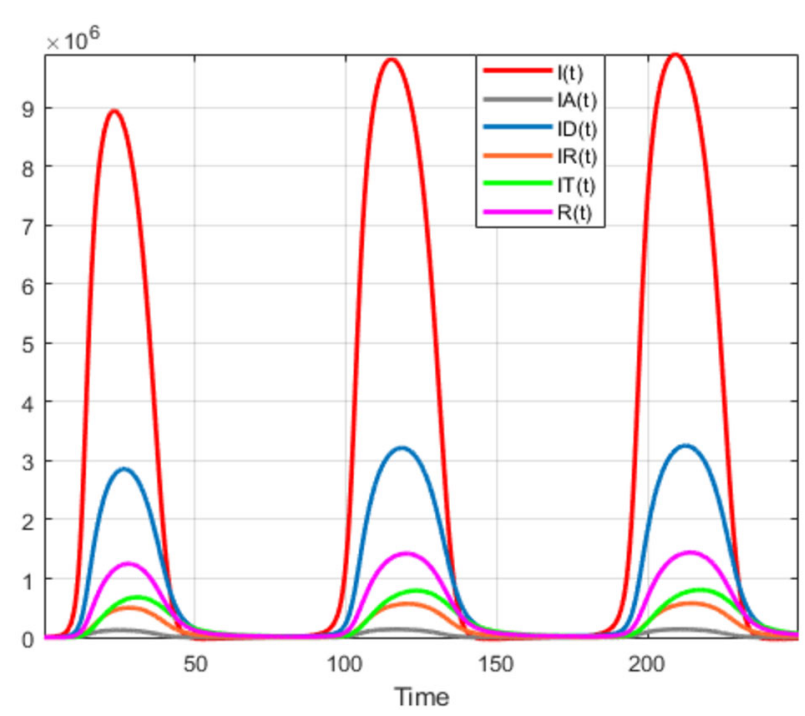

Figure 33 Numerical visualization of COVID-19 model for $\alpha=0.82$

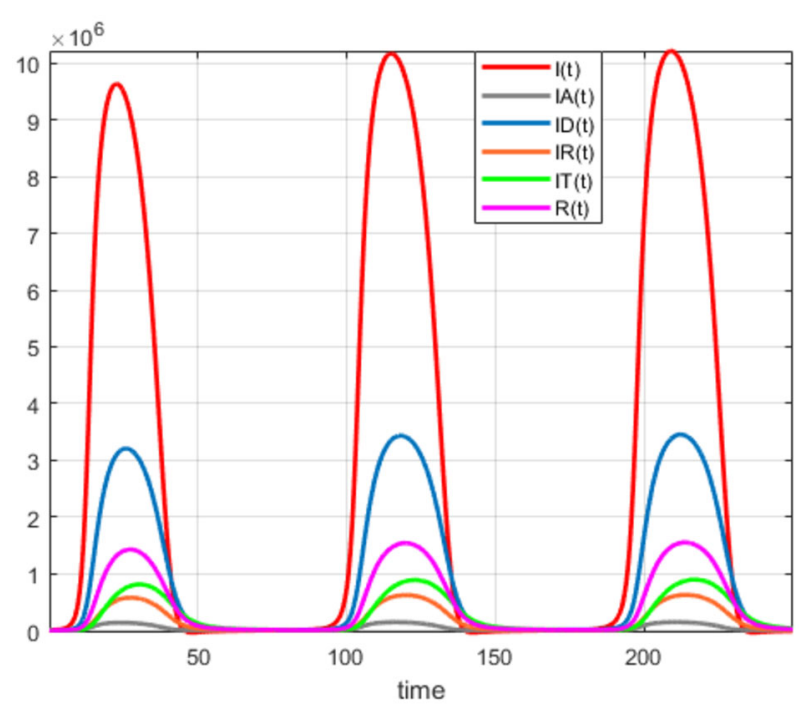

Figure 34 Numerical visualization of COVID-19 model for $\alpha=0.90$

$$
\begin{aligned}
& \sigma=0.015, \quad \delta_{0}=0.99, \quad \Delta t=900, \quad t_{0}=30, \quad \delta_{2}=0.4, \quad w=0.4, \\
& b=0.2, \quad a_{n}=0.1, \quad a_{r}=0.2, \quad a_{t}=0.3, \quad d_{0}=0.02, \quad d_{1}=0.2, \\
& d_{2}=0.15 .
\end{aligned}
$$

\section{Likelihood with hyper-Poisson distribution}

Using the suggested numerical model, we obtain the approximate solution $\left(S^{*}(t), I^{*}(t)\right.$, $\left.I_{A}^{*}(t), I_{D}^{*}(t), I_{R}^{*}(t), I_{T}^{*}(t), R^{*}(t), D^{*}(t), V^{*}(t)\right)$. We are more interested in $I^{*}(t), R^{*}(t)$, and $D^{*}(t)$ and the approximate solution $I, R, D$ because we have the collected data $z_{I}^{t}, z_{R}^{t}, z_{D}^{t}$ which represent the number of infections, recovered, and deaths daily. We assume that such follow hyper-Poisson distribution with parameters. The hyper-Poisson distribution is given 


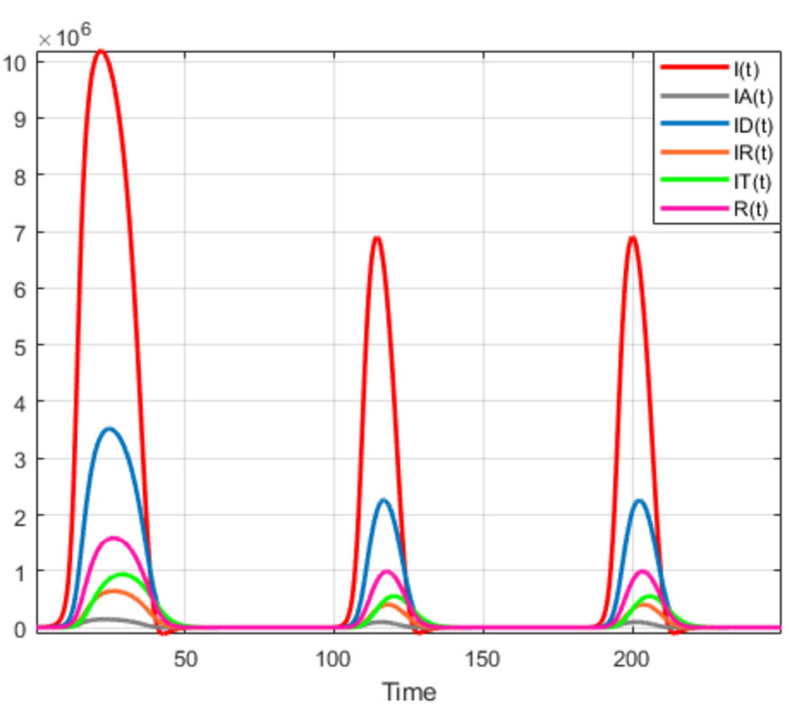

Figure 35 Numerical visualization of COVID-19 model for $\alpha=1$

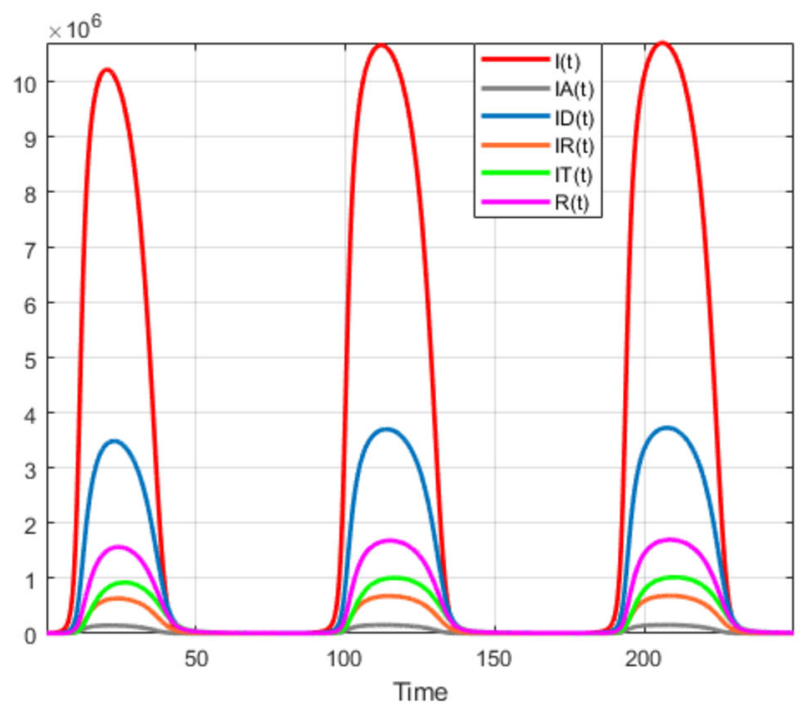

Figure 36 Numerical visualization of COVID-19 model for $\alpha=0.89, \beta=0.85$

as follows:

$$
P(X=k)=\frac{\Gamma(\beta)}{\Gamma(k+\beta) \Phi(1, \beta, \lambda)}, \quad \lambda>0, k=0,1,2, \ldots, n,
$$

where

$$
\Phi(1, \beta, \lambda)=\sum_{k=0}^{\infty} \frac{(1)_{k} \lambda^{k}}{(\beta)_{k} k !}, \quad(\beta)_{k}=\beta(\beta+1) \cdots(\beta+k)
$$




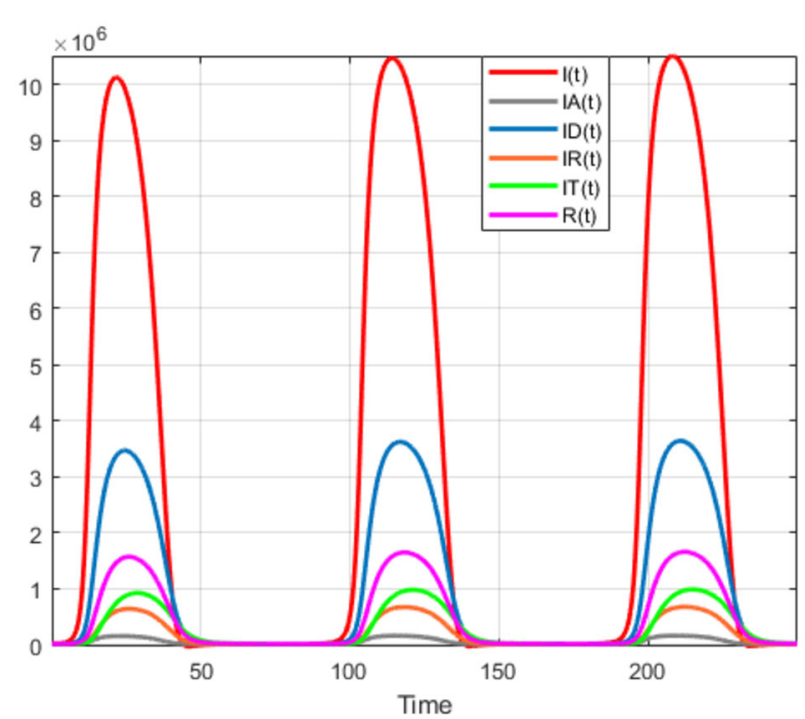

Figure 37 Numerical visualization of COVID-19 model for $\alpha=0.95, \beta=0.97$

$\Omega_{\prime \prime}^{\prime \prime}$ with parameters $k_{1}, k_{2}, k_{3}$

$$
\begin{aligned}
& k_{1}=\Omega_{1} I^{*}(t), \\
& k_{2}=\Omega_{2} R^{*}(t), \\
& k_{3}=\Omega_{3} D^{*}(t)
\end{aligned}
$$

and

$$
\begin{aligned}
& z_{I}^{t} \sim H P\left(k_{1}=\Omega_{1} I^{*}(t)\right), \\
& z_{R}^{t} \sim H P\left(k_{2}=\Omega_{2} R^{*}(t)\right), \\
& z_{D}^{t} \sim H P\left(k_{3}=\Omega_{3} D^{*}(t)\right) .
\end{aligned}
$$

Here, the parameters $\Omega_{1}, \Omega_{2}$, and $\Omega_{3}$ are a combination of collection accuracy and detectability of infected, recovered, and dead. Thus the likelihood function is defined as follows:

$$
\begin{aligned}
& L\left(k_{1}\right)=\prod_{t=0}^{n} g\left(z_{I}^{t} / k_{1}\right), \\
& L\left(k_{2}\right)=\prod_{t=0}^{n} g\left(z_{R}^{t} / k_{2}\right), \\
& L\left(k_{3}\right)=\prod_{t=0}^{n} g\left(z_{D}^{t} / k_{3}\right) .
\end{aligned}
$$

Thus

$$
L\left(k_{1}\right)=\prod_{t=0}^{n} \frac{\Gamma(\beta) \lambda_{I}^{t}}{\Gamma\left(z_{I}^{t}+\beta\right) \Phi(1, \beta, \lambda)},
$$




$$
\begin{aligned}
& L\left(k_{2}\right)=\prod_{t=0}^{n} \frac{\Gamma(\beta) \lambda^{z_{R}^{t}}}{\Gamma\left(z_{R}^{t}+\beta\right) \Phi(1, \beta, \lambda)}, \\
& L\left(k_{3}\right)=\prod_{t=0}^{n} \frac{\Gamma(\beta) \lambda^{z_{D}^{t}}}{\Gamma\left(z_{D}^{t}+\beta\right) \Phi(1, \beta, \lambda)} .
\end{aligned}
$$

Without loss of generality, we consider $L\left(k_{1}\right)$ :

$$
\begin{aligned}
\log L\left(k_{1}\right) & =\sum_{t=0}^{n} \log \frac{\Gamma(\beta) \lambda_{I}^{t}}{\Gamma\left(z_{I}^{t}+\beta\right) \Phi(1, \beta, \lambda)} \\
& =\sum_{t=0}^{n}\left[\log \Gamma(\beta)+z_{I}^{t} \log \left(\Omega_{1} I^{*}\right)-\log \Gamma\left(z_{I}^{t}+\beta\right)-\log \Phi\left(1, \beta, \Omega_{1} I^{*}\right)\right]
\end{aligned}
$$

and

$$
\begin{aligned}
& \frac{\partial \log L\left(k_{1}\right)}{\partial z_{I}^{t}}=\sum_{t=0}^{n} \log \left(\Omega_{1}\right)+\sum_{t=0}^{n} \log \left(I^{*}\right)-\sum_{t=0}^{n} \frac{\left(\Gamma\left(z_{I}^{t}+\beta\right)\right) \prime}{\Gamma\left(z_{I}^{t}+\beta\right)} \\
& =n\left[\log \left(\Omega_{1}\right)+\log \left(I^{*}\right)\right]-\sum_{t=0}^{n} \frac{\left(\Gamma\left(z_{I}^{t}+\beta\right)\right) \prime}{\Gamma\left(z_{I}^{t}+\beta\right)} \\
& =n \log \left(\Omega_{1} I^{*}\right)-\sum_{t=0}^{n} \frac{\left(\Gamma\left(z_{I}^{t}+\beta\right)\right) \prime}{\Gamma\left(z_{I}^{t}+\beta\right)}, \\
& \frac{\partial \log L\left(k_{1}\right)}{\partial I^{*}}=n z_{I}^{t} \frac{I^{*},}{I^{*}}-\sum_{t=0}^{n} \frac{\Phi\left(1, \beta, \Omega_{1} I^{*}\right) \prime}{\Phi\left(1, \beta, \Omega_{1} I^{*}\right)} \\
& =n z_{I}^{t} \frac{I^{* \prime}}{I^{*}}-n \frac{\Phi\left(1, \beta, \Omega_{1} I^{*}\right) \prime}{\Phi\left(1, \beta, \Omega_{1} I^{*}\right)}, \\
& \frac{\partial \log L\left(k_{1}\right)}{\partial \Omega_{1}}=n z_{I}^{t} \frac{\Omega_{1^{\prime}}}{\Omega_{1}}-n \frac{\Phi\left(1, \beta, \Omega_{1} I^{*}\right) \prime}{\Phi\left(1, \beta, \Omega_{1} I^{*}\right)} \\
& =-n \frac{\Phi\left(1, \beta, \Omega_{1} I^{*}\right) \prime}{\Phi\left(1, \beta, \Omega_{1} I^{*}\right)}, \\
& L\left(k_{2}\right)=\sum_{t=0}^{n} \log \frac{\Gamma(\beta) \lambda^{z_{R}^{t}}}{\Gamma\left(z_{R}^{t}+\beta\right) \Phi(1, \beta, \lambda)} \\
& =\sum_{t=0}^{n}\left[\log \Gamma(\beta)+z_{R}^{t} \log \lambda-\log \Gamma\left(z_{R}^{t}+\beta\right)-\log \Phi(1, \beta, \lambda)\right], \\
& \frac{\partial \log L\left(k_{2}\right)}{\partial z_{R}^{t}}=\sum_{t=0}^{n} \log \left(\Omega_{2}\right)+\sum_{t=0}^{n} \log \left(R^{*}\right)-\sum_{t=0}^{n} \frac{\left(\Gamma\left(z_{R}^{t}+\beta\right)\right) \prime}{\Gamma\left(z_{R}^{t}+\beta\right)} \\
& =n\left[\log \left(\Omega_{2}\right)+\log \left(R^{*}\right)\right]-\sum_{t=0}^{n} \frac{\left(\Gamma\left(z_{R}^{t}+\beta\right)\right) \prime}{\Gamma\left(z_{R}^{t}+\beta\right)} \\
& =n \log \left(\Omega_{2} R^{*}\right)-\sum_{t=0}^{n} \frac{\left(\Gamma\left(z_{R}^{t}+\beta\right)\right) \prime}{\Gamma\left(z_{R}^{t}+\beta\right)},
\end{aligned}
$$




$$
\begin{aligned}
& \frac{\partial \log L\left(k_{2}\right)}{\partial R^{*}}=n z_{R}^{t} \frac{R^{* \prime}}{R^{*}}-\sum_{t=0}^{n} \frac{\Phi\left(1, \beta, \Omega_{2} R^{*}\right) \prime}{\Phi\left(1, \beta, \Omega_{2} R^{*}\right)} \\
& =n z_{R}^{t} \frac{R^{* \prime}}{R^{*}}-n \frac{\Phi\left(1, \beta, \Omega_{2} R^{*}\right) \prime}{\Phi\left(1, \beta, \Omega_{2} R^{*}\right)}, \\
& \frac{\partial \log L\left(k_{2}\right)}{\partial \Omega_{2}}=n z_{R}^{t} \frac{\Omega_{2}^{\prime}}{\Omega_{2}}-n \frac{\Phi\left(1, \beta, \Omega_{2} R^{*}\right) \prime}{\Phi\left(1, \beta, \Omega_{2} R^{*}\right)} \\
& =-n \frac{\Phi\left(1, \beta, \Omega_{2} R^{*}\right) \prime}{\Phi\left(1, \beta, \Omega_{2} R^{*}\right)}, \\
& L\left(k_{3}\right)=\sum_{t=0}^{n} \log \frac{\Gamma(\beta) \lambda^{z_{D}^{t}}}{\Gamma\left(z_{D}^{t}+\beta\right) \Phi(1, \beta, \lambda)} \\
& =\sum_{t=0}^{n}\left[\log \Gamma(\beta)+z_{D}^{t} \log \lambda-\log \Gamma\left(z_{D}^{t}+\beta\right)-\log \Phi(1, \beta, \lambda)\right], \\
& \frac{\partial \log L\left(k_{3}\right)}{\partial z_{D}^{t}}=\sum_{t=0}^{n} \log \left(\Omega_{3}\right)+\sum_{t=0}^{n} \log \left(D^{*}\right)-\sum_{t=0}^{n} \frac{\left(\Gamma\left(z_{D}^{t}+\beta\right)\right) \prime}{\Gamma\left(z_{D}^{t}+\beta\right)} \\
& =n\left[\log \left(\Omega_{3}\right)+\log \left(D^{*}\right)\right]-\sum_{t=0}^{n} \frac{\left(\Gamma\left(z_{D}^{t}+\beta\right)\right) \prime}{\Gamma\left(z_{D}^{t}+\beta\right)} \\
& =n \log \left(\Omega_{3} D^{*}\right)-\sum_{t=0}^{n} \frac{\left(\Gamma\left(z_{D}^{t}+\beta\right)\right) \prime}{\Gamma\left(z_{D}^{t}+\beta\right)}, \\
& \frac{\partial \log L\left(k_{3}\right)}{\partial R^{*}}=n z_{D}^{t} \frac{D^{* \prime}}{D^{*}}-\sum_{t=0}^{n} \frac{\Phi\left(1, \beta, \Omega_{3} D^{*}\right) \prime}{\Phi\left(1, \beta, \Omega_{3} D^{*}\right)} \\
& =n z_{D}^{t} \frac{D^{* \prime}}{D^{*}}-n \frac{\Phi\left(1, \beta, \Omega_{3} D^{*}\right) \prime}{\Phi\left(1, \beta, \Omega_{3} D^{*}\right)}, \\
& \frac{\partial \log L\left(k_{3}\right)}{\partial \Omega_{3}}=n z_{D}^{t} \frac{\Omega_{3}^{\prime}}{\Omega_{3}}-n \frac{\Phi\left(1, \beta, \Omega_{3} D^{*}\right) \prime}{\Phi\left(1, \beta, \Omega_{3} D^{*}\right)} \\
& =-n \frac{\Phi\left(1, \beta, \Omega_{3} D^{*}\right) \prime}{\Phi\left(1, \beta, \Omega_{3} D^{*}\right)} \text {. }
\end{aligned}
$$

\section{Likelihood with Weibull distribution}

We will do the same routine for the Weibull distribution known as

$$
P(X=k)=\frac{k}{\alpha}\left(\frac{\lambda}{\alpha}\right)^{k-1} \exp (-\lambda / \alpha)^{k}, \quad \lambda, \alpha>0, k=0,1,2, \ldots, n,
$$

$\Omega$ with parameters $k_{1}, k_{2}, k_{3}$

$$
\begin{aligned}
& k_{1}=\Omega_{1} I^{*}(t), \\
& k_{2}=\Omega_{2} R^{*}(t), \\
& k_{3}=\Omega_{3} D^{*}(t)
\end{aligned}
$$

and

$$
\varepsilon_{I}^{t} \sim W\left(k_{1}=\Omega_{1} I^{*}(t)\right)
$$




$$
\begin{aligned}
& \varepsilon_{R}^{t} \sim W\left(k_{2}=\Omega_{2} R^{*}(t)\right), \\
& \varepsilon_{D}^{t} \sim W\left(k_{3}=\Omega_{3} D^{*}(t)\right) .
\end{aligned}
$$

Thus the likelihood function is given by

$$
\begin{aligned}
& L\left(k_{1}\right)=\prod_{t=0}^{n} W\left(\varepsilon_{I}^{t} / k_{1}\right), \\
& L\left(k_{2}\right)=\prod_{t=0}^{n} W\left(\varepsilon_{R}^{t} / k_{2}\right), \\
& L\left(k_{3}\right)=\prod_{t=0}^{n} W\left(\varepsilon_{D}^{t} / k_{3}\right) .
\end{aligned}
$$

Thus

$$
\begin{aligned}
& L\left(k_{1}\right)=\prod_{t=0}^{n} \frac{\varepsilon_{I}^{t}}{\alpha}\left(\frac{\lambda}{\alpha}\right)^{\varepsilon_{I}^{t}-1} \exp (-\lambda / \alpha)^{\varepsilon_{I}^{t}}, \\
& L\left(k_{2}\right)=\prod_{t=0}^{n} \frac{\varepsilon_{R}^{t}}{\alpha}\left(\frac{\lambda}{\alpha}\right)^{\varepsilon_{R}^{t}-1} \exp (-\lambda / \alpha)^{\varepsilon_{R}^{t}}, \\
& L\left(k_{3}\right)=\prod_{t=0}^{n} \frac{\varepsilon_{D}^{t}}{\alpha}\left(\frac{\lambda}{\alpha}\right)^{\varepsilon_{D}^{t}-1} \exp (-\lambda / \alpha)^{\varepsilon_{D}^{t}} .
\end{aligned}
$$

Without loss of generality, we consider $L\left(k_{1}\right)$ :

$$
\begin{aligned}
\log L\left(k_{1}\right) & =\sum_{t=0}^{n} \log \left[\frac{\varepsilon_{I}^{t}}{\alpha}\left(\frac{\Omega_{1} I^{*}}{\alpha}\right)^{\varepsilon_{I}^{t}-1} \exp \left(-\Omega_{1} I^{*} / \alpha\right)^{\varepsilon_{I}^{t}}\right] \\
& =\left[\log \varepsilon_{I}^{t}-\log \alpha+\left(\varepsilon_{I}^{t}-1\right)\left[\log \left(\Omega_{1} I^{*}\right)-\log \alpha\right]-\varepsilon_{I}^{t}\left(-\Omega_{1} I^{*} / \alpha\right)\right]
\end{aligned}
$$

and

$$
\begin{aligned}
\frac{\partial \log L\left(k_{1}\right)}{\partial \varepsilon_{I}^{t}} & =\sum_{t=0}^{n} \frac{\varepsilon_{I}^{t}}{\varepsilon_{I}^{t}}+\sum_{t=0}^{n}\left[\log \left(\Omega_{1} I^{*}\right)-\log \alpha\right]-\sum_{t=0}^{n}\left(-\Omega_{1} I^{*} / \alpha\right) \\
& =n \frac{\varepsilon_{I}^{t} \prime}{\varepsilon_{I}^{t}}+n\left[\log \left(\Omega_{1} I^{*}\right)-\log \alpha\right]+n\left(\Omega_{1} I^{*} / \alpha\right), \\
\frac{\partial \log L\left(k_{1}\right)}{\partial I^{*}} & =n\left(\varepsilon_{I}^{t}-1\right) \frac{I^{* \prime}}{I^{*}}-\sum_{t=0}^{n} \frac{\left(-\Omega_{1} I^{*} / \alpha\right) \prime}{\left(-\Omega_{1} I^{*} / \alpha\right)} \\
& =n\left(\varepsilon_{I}^{t}-1\right) \frac{I^{* \prime}}{I^{*}}-n \frac{\left(-\Omega_{1} I^{*} / \alpha\right) \prime}{\left(-\Omega_{1} I^{*} / \alpha\right)}, \\
\frac{\partial \log L\left(k_{1}\right)}{\partial \Omega_{1}} & =n\left(\varepsilon_{I}^{t}-1\right) \frac{\Omega_{1}^{\prime}}{\Omega_{1}}-n \frac{\left(-\Omega_{1} I^{*} / \alpha\right) \prime}{\left(-\Omega_{1} I^{*} / \alpha\right)} \\
& =-n \frac{\left(-\Omega_{1} I^{*} / \alpha\right) \prime}{\left(-\Omega_{1} I^{*} / \alpha\right)},
\end{aligned}
$$




$$
\begin{aligned}
\log L\left(k_{2}\right) & =\sum_{t=0}^{n} \log \left[\frac{\varepsilon_{R}^{t}}{\alpha}\left(\frac{\Omega_{2} I^{*}}{\alpha}\right)^{\varepsilon_{R}^{t}-1} \exp \left(-\Omega_{2} R^{*} / \alpha\right)^{\varepsilon_{I}^{t}}\right] \\
& =\left[\log \varepsilon_{R}^{t}-\log \alpha+\left(\varepsilon_{R}^{t}-1\right)\left[\log \left(\Omega_{2} R^{*}\right)-\log \alpha\right]-\varepsilon_{R}^{t}\left(-\Omega_{2} R^{*} / \alpha\right)\right]
\end{aligned}
$$

and

$$
\begin{aligned}
\frac{\partial \log L\left(k_{2}\right)}{\partial \varepsilon_{R}^{t}} & =\sum_{t=0}^{n} \frac{\varepsilon_{R^{\prime}}^{t}}{\varepsilon_{R}^{t}}+\sum_{t=0}^{n}\left[\log \left(\Omega_{2} R^{*}\right)-\log \alpha\right]-\sum_{t=0}^{n}\left(-\Omega_{2} R^{*} / \alpha\right) \\
& =n \frac{\varepsilon_{R}^{t} R^{\prime}}{\varepsilon_{R}^{t}}+n\left[\log \left(\Omega_{2} R^{*}\right)-\log \alpha\right]+n\left(-\Omega_{2} R^{*} / \alpha\right), \\
\frac{\partial \log L\left(k_{2}\right)}{\partial R^{*}} & =n\left(\varepsilon_{R}^{t}-1\right) \frac{R^{* \prime}}{R^{*}}-\sum_{t=0}^{n} \frac{\left(-\Omega_{2} R^{*} / \alpha\right) \prime}{\left(-\Omega_{2} R^{*} / \alpha\right)} \\
& =n\left(\varepsilon_{R}^{t}-1\right) \frac{R^{* \prime}}{R^{*}}-n \frac{\left(-\Omega_{2} R^{*} / \alpha\right) \prime}{\left(-\Omega_{2} R^{*} / \alpha\right)} \\
\frac{\partial \log L\left(k_{2}\right)}{\partial \Omega_{2}} & =n\left(\varepsilon_{R}^{t}-1\right) \frac{\Omega_{2} \prime}{\Omega_{2}}-n \frac{\left(-\Omega_{2} R^{*} / \alpha\right) \prime}{\left(-\Omega_{2} R^{*} / \alpha\right)} \\
& =-n \frac{\left(-\Omega_{2} R^{*} / \alpha\right) \prime}{\left(-\Omega_{2} R^{*} / \alpha\right)}, \\
\log L\left(k_{3}\right)= & \sum_{t=0}^{n} \log \left[\frac{\varepsilon_{D}^{t}}{\alpha}\left(\frac{\Omega_{3} D^{*}}{\alpha}\right)^{\varepsilon_{D}^{t}-1} \exp \left(-\Omega_{3} D^{*} / \alpha\right)^{\varepsilon_{D}^{t}}\right] \\
= & {\left[\log \varepsilon_{D}^{t}-\log \alpha+\left(\varepsilon_{D}^{t}-1\right)\left[\log \left(\Omega_{3} D^{*}\right)-\log \alpha\right]-\varepsilon_{D}^{t}\left(-\Omega_{3} D^{*} / \alpha\right)\right] }
\end{aligned}
$$

and

$$
\begin{aligned}
\frac{\partial \log L\left(k_{3}\right)}{\partial \varepsilon_{D}^{t}} & =\sum_{t=0}^{n} \frac{\varepsilon_{D^{\prime}}^{t}}{\varepsilon_{D}^{t}}+\sum_{t=0}^{n}\left[\log \left(\Omega_{3} D^{*}\right)-\log \alpha\right]-\sum_{t=0}^{n}\left(-\Omega_{3} D^{*} / \alpha\right) \\
& =n \frac{\varepsilon_{D^{\prime}}^{t}}{\varepsilon_{D}^{t}}+n\left[\log \left(\Omega_{3} R^{*}\right)-\log \alpha\right]+n\left(-\Omega_{3} D^{*} / \alpha\right), \\
\frac{\partial \log L\left(k_{3}\right)}{\partial D^{*}} & =n\left(\varepsilon_{D}^{t}-1\right) \frac{D^{* \prime}}{D^{*}}-\sum_{t=0}^{n} \frac{\left(-\Omega_{3} D^{*} / \alpha\right) \prime}{\left(-\Omega_{3} D^{*} / \alpha\right)} \\
& =n\left(\varepsilon_{I}^{t}-1\right) \frac{D^{* \prime}}{D^{*}}-n \frac{\left(-\Omega_{3} D^{*} / \alpha\right) \prime}{\left(-\Omega_{3} D^{*} / \alpha\right)} \\
\frac{\partial \log L\left(k_{1}\right)}{\partial \Omega_{1}} & =n\left(\varepsilon_{D}^{t}-1\right) \frac{\Omega_{3} \prime}{\Omega_{3}}-n \frac{\left(-\Omega_{3} D^{*} / \alpha\right) \prime}{\left(-\Omega_{3} D^{*} / \alpha\right)} \\
& =-n \frac{\left(-\Omega_{3} D^{*} / \alpha\right) \prime}{\left(-\Omega_{3} D^{*} / \alpha\right)} .
\end{aligned}
$$

\section{Likelihood with Mittag-Leffler distribution}

Finally, we shall use the Mittag-Leffler distribution for similar processes. The MittagLeffler distribution is defined by

$$
P(X=k)=\frac{\lambda^{k}}{\Gamma(\alpha k+\beta) E_{\alpha, \beta}(\lambda)}, \quad \lambda>0, k=0,1,2, \ldots, n,
$$


where

$$
E_{\alpha, \beta}(\lambda)=\sum_{k=0}^{\infty} \frac{\lambda^{k}}{\Gamma(\alpha k+\beta)} .
$$

$\Omega_{i}, i=1,2,3$ with parameters $k_{1}, k_{2}, k_{3}$

$$
\begin{aligned}
& k_{1}=\Omega_{1} I^{*}(t), \\
& k_{2}=\Omega_{2} R^{*}(t), \\
& k_{3}=\Omega_{3} D^{*}(t)
\end{aligned}
$$

and

$$
\begin{aligned}
& \varepsilon_{I}^{t} \sim M L\left(k_{1}=\Omega_{1} I^{*}(t)\right), \\
& \varepsilon_{R}^{t} \sim M L\left(k_{2}=\Omega_{2} R^{*}(t)\right), \\
& \varepsilon_{D}^{t} \sim M L\left(k_{3}=\Omega_{3} D^{*}(t)\right) .
\end{aligned}
$$

Thus the likelihood function is written as

$$
\begin{aligned}
& L\left(k_{1}\right)=\prod_{t=0}^{n} M L\left(\varepsilon_{I}^{t} / k_{1}\right), \\
& L\left(k_{2}\right)=\prod_{t=0}^{n} M L\left(\varepsilon_{R}^{t} / k_{2}\right), \\
& L\left(k_{3}\right)=\prod_{t=0}^{n} M L\left(\varepsilon_{D}^{t} / k_{3}\right) .
\end{aligned}
$$

Thus

$$
\begin{aligned}
& L\left(k_{1}\right)=\prod_{t=0}^{n} \frac{\lambda^{\varepsilon_{I}^{t}}}{\Gamma\left(\alpha \varepsilon_{I}^{t}+\beta\right) E_{\alpha, \beta}(\lambda)}, \\
& L\left(k_{2}\right)=\prod_{t=0}^{n} \frac{\lambda^{\varepsilon_{R}^{t}}}{\Gamma\left(\alpha \varepsilon_{R}^{t}+\beta\right) E_{\alpha, \beta}(\lambda)}, \\
& L\left(k_{3}\right)=\prod_{t=0}^{n} \frac{\lambda^{\varepsilon_{D}^{t}}}{\Gamma\left(\alpha \varepsilon_{D}^{t}+\beta\right) E_{\alpha, \beta}(\lambda)} .
\end{aligned}
$$

We write $L\left(k_{1}\right)$ :

$$
\begin{aligned}
\log L\left(k_{1}\right) & =\log \frac{\lambda^{\varepsilon_{I}^{t}}}{\Gamma\left(\alpha \varepsilon_{I}^{t}+\beta\right) E_{\alpha, \beta}(\lambda)} \\
& =\sum_{t=0}^{n}\left[\varepsilon_{I}^{t} \log \left(\Omega_{1} I^{*}\right)-\log \Gamma\left(\alpha \varepsilon_{I}^{t}+\beta\right)-\log E_{\alpha, \beta}\left(\Omega_{1} I^{*}\right)\right]
\end{aligned}
$$


and

$$
\begin{aligned}
\frac{\partial \log L\left(k_{1}\right)}{\partial \varepsilon_{I}^{t}} & =\sum_{t=0}^{n} \log \left(\Omega_{1}\right)+\sum_{t=0}^{n} \log \left(I^{*}\right)-\sum_{t=0}^{n} \frac{\left(\Gamma\left(\alpha \varepsilon_{I}^{t}+\beta\right)\right) \prime}{\Gamma\left(\alpha \varepsilon_{I}^{t}+\beta\right)} \\
& =n\left[\log \left(\Omega_{1}\right)+\log \left(I^{*}\right)\right]-\sum_{t=0}^{n} \frac{\left(\Gamma\left(\alpha \varepsilon_{I}^{t}+\beta\right)\right) \prime}{\Gamma\left(\alpha \varepsilon_{I}^{t}+\beta\right)} \\
& =n \log \left(\Omega_{1} I^{*}\right)-\sum_{t=0}^{n} \frac{\left(\Gamma\left(\alpha \varepsilon_{I}^{t}+\beta\right)\right) \prime}{\Gamma\left(\alpha \varepsilon_{I}^{t}+\beta\right)} \\
\frac{\partial \log L\left(k_{1}\right)}{\partial I^{*}} & =n \varepsilon_{I}^{t} \frac{I^{* \prime}}{I^{*}}-\sum_{t=0}^{n} \frac{E_{\alpha, \beta}\left(\Omega_{1} I^{*}\right) \prime}{E_{\alpha, \beta}\left(\Omega_{1} I^{*}\right)} \\
& =n \varepsilon_{I}^{t} \frac{I^{* \prime}}{I^{*}}-n \frac{E_{\alpha, \beta}\left(\Omega_{1} I^{*}\right) \prime}{E_{\alpha, \beta}\left(\Omega_{1} I^{*}\right)} \\
\frac{\partial \log L\left(k_{1}\right)}{\partial \Omega_{1}} & =n \varepsilon_{I}^{t} \frac{\Omega_{1^{\prime}}}{\Omega_{1}}-n \frac{E_{\alpha, \beta}\left(\Omega_{1} I^{*}\right) \prime}{E_{\alpha, \beta}\left(\Omega_{1} I^{*}\right)} \\
& =-n \frac{E_{\alpha, \beta}\left(\Omega_{1} I^{*}\right) \prime}{E_{\alpha, \beta}\left(\Omega_{1} I^{*}\right)} .
\end{aligned}
$$

With the same routine,

$$
\begin{aligned}
\log L\left(k_{2}\right) & =\sum_{t=0}^{n} \log \frac{\lambda^{\varepsilon_{R}^{t}}}{\Gamma\left(\alpha \varepsilon_{I}^{t}+\beta\right) E_{\alpha, \beta}(\lambda)} \\
& =\sum_{t=0}^{n}\left[\varepsilon_{R}^{t} \log \left(\Omega_{1} R^{*}\right)-\log \Gamma\left(\alpha \varepsilon_{R}^{t}+\beta\right)-\log E_{\alpha, \beta}\left(\Omega_{2} R^{*}\right)\right]
\end{aligned}
$$

and

$$
\begin{aligned}
\frac{\partial \log L\left(k_{2}\right)}{\partial \varepsilon_{R}^{t}} & =\sum_{t=0}^{n} \log \left(\Omega_{2}\right)+\sum_{t=0}^{n} \log \left(R^{*}\right)-\sum_{t=0}^{n} \frac{\left(\Gamma\left(\alpha \varepsilon_{R}^{t}+\beta\right)\right) \prime}{\Gamma\left(\alpha \varepsilon_{R}^{t}+\beta\right)} \\
& =n\left[\log \left(\Omega_{2}\right)+\log \left(R^{*}\right)\right]-\sum_{t=0}^{n} \frac{\left(\Gamma\left(\alpha \varepsilon_{R}^{t}+\beta\right)\right) \prime}{\Gamma\left(\alpha \varepsilon_{R}^{t}+\beta\right)} \\
& =n \log \left(\Omega_{2} R^{*}\right)-\sum_{t=0}^{n} \frac{\left(\Gamma\left(\alpha \varepsilon_{R}^{t}+\beta\right)\right) \prime}{\Gamma\left(\alpha \varepsilon_{R}^{t}+\beta\right)} \\
\frac{\partial \log L\left(k_{2}\right)}{\partial R^{*}} & =n \varepsilon_{R}^{t} \frac{R^{* \prime}}{R^{*}}-\sum_{t=0}^{n} \frac{E_{\alpha, \beta}\left(\Omega_{2} R^{*}\right) \prime}{E_{\alpha, \beta}\left(\Omega_{2} R^{*}\right)} \\
& =n \varepsilon_{I}^{t} \frac{R^{* \prime}}{R^{*}}-n \frac{E_{\alpha, \beta}\left(\Omega_{2} R^{*}\right) \prime}{E_{\alpha, \beta}\left(\Omega_{2} R^{*}\right)} \\
\frac{\partial \log L\left(k_{2}\right)}{\partial \Omega_{2}} & =n \varepsilon_{R}^{t} \frac{\Omega_{2^{\prime}}}{\Omega_{2}}-n \frac{E_{\alpha, \beta}\left(\Omega_{2} R^{*}\right) \prime}{E_{\alpha, \beta}\left(\Omega_{2} R^{*}\right)} \\
& =-n \frac{E_{\alpha, \beta}\left(\Omega_{2} R^{*}\right) \prime}{E_{\alpha, \beta}\left(\Omega_{2} R^{*}\right)}
\end{aligned}
$$


and

$$
\begin{aligned}
\log L\left(k_{3}\right) & =\sum_{t=0}^{n} \log \frac{\lambda^{t_{D}^{t}}}{\Gamma\left(\alpha \varepsilon_{D}^{t}+\beta\right) E_{\alpha, \beta}(\lambda)} \\
& =\sum_{t=0}^{n}\left[\varepsilon_{D}^{t} \log \left(\Omega_{3} D^{*}\right)-\log \Gamma\left(\alpha \varepsilon_{D}^{t}+\beta\right)-\log E_{\alpha, \beta}\left(\Omega_{3} D^{*}\right)\right]
\end{aligned}
$$

and

$$
\begin{aligned}
\frac{\partial \log L\left(k_{1}\right)}{\partial \varepsilon_{I}^{t}} & =\sum_{t=0}^{n} \log \left(\Omega_{3}\right)+\sum_{t=0}^{n} \log \left(D^{*}\right)-\sum_{t=0}^{n} \frac{\left(\Gamma\left(\alpha \varepsilon_{D}^{t}+\beta\right)\right) \prime}{\Gamma\left(\alpha \varepsilon_{D}^{t}+\beta\right)} \\
& =n\left[\log \left(\Omega_{3}\right)+\log \left(D^{*}\right)\right]-\sum_{t=0}^{n} \frac{\left(\Gamma\left(\alpha \varepsilon_{D}^{t}+\beta\right)\right) \prime}{\Gamma\left(\alpha \varepsilon_{D}^{t}+\beta\right)} \\
& =n \log \left(\Omega_{3} D^{*}\right)-\sum_{t=0}^{n} \frac{\left(\Gamma\left(\alpha \varepsilon_{D}^{t}+\beta\right)\right) \prime}{\Gamma\left(\alpha \varepsilon_{D}^{t}+\beta\right)} \\
\frac{\partial \log L\left(k_{3}\right)}{\partial D^{*}} & =n \varepsilon_{D}^{t} \frac{D^{* \prime}}{D^{*}}-\sum_{t=0}^{n} \frac{E_{\alpha, \beta}\left(\Omega_{3} D^{*}\right) \prime}{E_{\alpha, \beta}\left(\Omega_{3} D^{*}\right)} \\
& =n \varepsilon_{D}^{t} \frac{D^{* \prime}}{D^{*}}-n \frac{E_{\alpha, \beta}\left(\Omega_{3} D^{*}\right) \prime}{E_{\alpha, \beta}\left(\Omega_{3} D^{*}\right)} \\
\frac{\partial \log L\left(k_{3}\right)}{\partial \Omega_{3}} & =n \varepsilon_{D}^{t} \frac{\Omega_{1^{\prime}}}{\Omega_{1}}-n \frac{E_{\alpha, \beta}\left(\Omega_{3} D^{*}\right) \prime}{E_{\alpha, \beta}\left(\Omega_{3} D^{*}\right)} \\
& =-n \frac{E_{\alpha, \beta}\left(\Omega_{3} D^{*}\right) \prime}{E_{\alpha, \beta}\left(\Omega_{3} D^{*}\right)} .
\end{aligned}
$$

\section{Conclusion}

Up to date humans have relied on forecasting with the aim to better control their world, or at least to have an asymptotic idea of their future. They have many ways to achieve this, one way is to use the deterministic approach and another is stochastic one. In this work, we presented a comprehensive analysis ranging from stochastic, fractal to differentiation with the aim to predict the future behavior of COVID-19 with cases studied in Africa and Europe. With stochastic approach, we were able to detect a possibility of the second wave of COVID-19 spread in Europe and in Africa, a continuous exponential growth could be possible. We presented an extension of the blancmange function to capture more fractal behaviors, and some examples were presented resembling the COVID-19 spread in various countries in Africa and Europe. A complex and nonlinear mathematical model with wave function was considered and solved numerically with a modified scheme.

\section{Acknowledgements}

The authors of this paper would like to thank the referees for their valuable suggestions and comments.

Funding

There is no funding for this paper.

Availability of data and materials

There are no data for this paper.

Competing interests

The authors declare that they have no competing interests. 
Authors' contributions

All authors have contributed equally in this work. All authors read and approved the final manuscript.

\section{Author details}

${ }^{1}$ Institute for Groundwater Studies, Faculty of Natural and Agricultural Sciences, University of the Free State, Bloemfontein South Africa. ${ }^{2}$ Department of Medical Research, China Medical University Hospital, China Medical University, Taichung, Taiwan. ${ }^{3}$ Department of Mathematic Education, Faculty of Education, Siirt University, Siirt 56100, Turkey.

\section{Publisher's Note}

Springer Nature remains neutral with regard to jurisdictional claims in published maps and institutional affiliations.

Received: 5 November 2020 Accepted: 4 January 2021 Published online: 20 January 2021

\section{References}

1. WHO: Coronavirus disease (Covid-2019) situation reports.

https://www.who.int/emergencies/diseases/novel-coronavirus-2019/situation-reports

2. https://who.maps.arcgis.com/apps/opsdashboard/index.html\#/ead3c6475654481ca51c248d52ab9c61

3. Ndaïrou, F., Area, I., Nieto, J.J., Torres, D.F.M.: Mathematical modeling of Covid-19 transmission dynamics with a case study of Wuhan. Chaos Solitons Fractals 135, 109846 (2020)

4. Khan, M.A., Atangana, A.: Modeling the dynamics of novel coronavirus (2019-nCov) with fractional derivative. Alex. Eng. J. 59(4), 2379-2389 (2020)

5. Postnikov, E.B.: Estimation of Covid-19 dynamics "on a back-of-envelope": does the simplest SIR model provide quantitative parameters and predictions? Chaos Solitons Fractals 135, 109841 (2020)

6. Atangana, A., İgret Araz, S.: Nonlinear equations with global differential and integral operators: existence, uniqueness with application to epidemiology. Results Phys., 20, 103593 (2020)

7. Jiao, J., Liu, Z., Cai, S.: Dynamics of an SEIR model with infectivity in incubation period and homestead-isolation on the susceptible. Appl. Math. Lett. 107, 106442 (2020)

8. Atangana, A., İgret Araz, S.: Mathematical model of Covid-19 spread in Turkey and South Africa: theory, methods and applications. medRxiv (2020)

9. Ivorra, B., Ferrández, M.R., Vela-Péreza, M., Ramos, A.M.: Mathematical modeling of the spread of the coronavirus disease 2019 (Covid-19) taking into account the undetected infections. The case of China. Commun. Nonlinear Sci. Numer. Simul. 88, 105303 (2020)

10. Rezapour, S., Mohammadi, H., Jajarmi, A.: A new mathematical model for Zika virus transmission. Adv. Differ. Equ. 2020, 589 (2020)

11. Atangana, A.: Modelling the spread of COVID-19 with new fractal-fractional operators: can the lockdown save mankind before vaccination? Chaos Solitons Fractals 136, 109860 (2020)

12. Jajarmi, A., Baleanu, D.: A new iterative method for the numerical solution of high-order nonlinear fractional boundary value problems. Front. Phys. 8, 220 (2020)

13. Atangana, A., Iğret Araz, S.: Analysis of a Covid-19 model: optimal control, stability and simulations. Alex. Eng. J. 60(1) 647-658 (2021)

14. Singh, S., Parmar, K.S., Singh Makkhan, S.J., Kaur, J., Peshoria, S., Kumar, J.: Study of ARIMA and least square support vector machine (LS-SVM) models for the prediction of SARS-CoV-2 confirmed cases in the most affected countries. Chaos Solitons Fractals 139, 110086 (2020)

15. Barnsley, M.: Fractals Everywhere, 3rd edn. Dover, New York (2012)

16. Păcurar, C.M., Necula, B.R.: An analysis of Covid-19 spread based on fractal interpolation and fractal dimension. Chaos Solitons Fractals 139, 110073 (2020)

17. Ovidiu, C.: An Informal Introduction to Stochastic Calculus with Applications. World Scientific, Singapore (2015). p. 315. ISBN 978-981-4678-93-3

18. Atangana, A., Araz, S.I.: New Numerical Scheme with Newton Polynomial: Theory, Methods, and Applications. Academic Press, San Diego (2021). ISBN 9780323854481

\section{Submit your manuscript to a SpringerOpen ${ }^{\circ}$ journal and benefit from:}

- Convenient online submission

- Rigorous peer review

- Open access: articles freely available online

- High visibility within the field

- Retaining the copyright to your article

Submit your next manuscript at $>$ springeropen.com 